$$
\text { USGS/OFR--98-23/ }
$$

U.S. DEPARTMENT OF THE INTERIOR

U.S. GEOLOGICAL SURVEY

\title{
AUDIO-MAGNETOTELLURIC DATA COLLECTED IN THE BEATTY, NEVADA AREA
}

By

Jackie M. Williams

Denver, Colorado

Open-File Report 98-231

PECEIVED

1998

SEP 1.69998

OSTI

This report is preliminary and has not been edited or reviewed for conformity with the U.S. Geological Survey editorial standards. Any use of trade names is for descriptive purposes only and does not imply endorsement by the U.S. Geological Survey.

AI08-96NVI1967

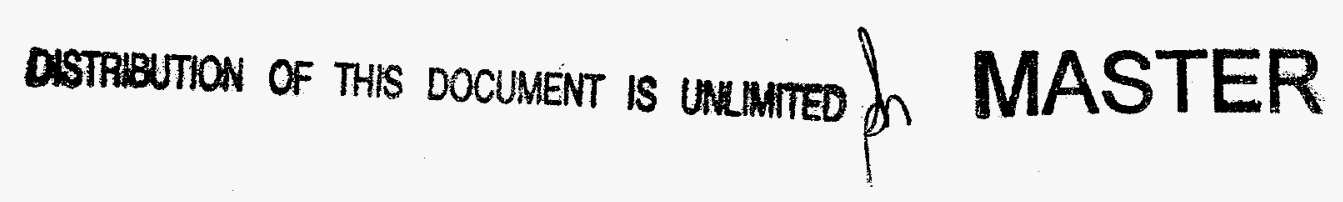




\section{DISCLAIMER}

This report was prepared as an account of work sponsored by an agency of the United States Government. Neither the United States Government nor any agency thereof, nor any of their employees, makes any warranty, express or implied, or assumes any legal liability or responsibility for the accuracy, completeness, or usefulness of any information, apparatus, product, or process disclosed, or represents that its use would not infringe privately owned rights. Reference herein to any specific commercial product, process, or service by trade name, trademark, manufacturer, or otherwise does not necessarily constitute or imply its endorsement, recommendation, or favoring by the United States Government or any agency thereof. The views and opinions of authors expressed herein do not necessarily state or reflect those of the United States Government or any agency thereof. 


\section{DISCLAIMER}

Portions of this document may be illegible in electronic image products. Images are produced from the best available original document. 


\title{
AUDIO-MAGNETOTELLURIC DATA COLLECTED IN THE AREA OF BEATTY, NEVADA
}

\author{
Jackie M. Williams, \\ U.S. Geological Survey \\ 1997
}

\section{INTRODUCTION}

In the summer of 1997, electrical geophysical data was collected north of Beatty, Nevada. Audio-magnetotellurics (AMT) was the geophysical method used to collect 16 stations along two (2) profiles. The purpose of this data collection was to determine the depth to the alluvial basement, based upon the needs of the geologists requesting the data.

\section{DATA}

The data was collected using the MT-1 magnetotelluric system (equipment and computer programs) of ElectroMagnetic Instruments, Inc. This system employs simultaneous orthogonal electric field and magnetic field measurements along with a remote station of either an electrical or magnetic field. We used a local remote that was less than 100 meters from the main station setup. This remote station allows for remote-reference noise cancellation which helps prevent bias of the data from noise created by power lines, radio antenna, or other cultural frequencies. Also, a vertical magnetic coil was used to collect tipper data which allows us to better determine the electrical strike direction.

Data was collected over five (5) ranges of frequency bands. The frequency bands used were, $1-170 \mathrm{~Hz}, 79-580 \mathrm{~Hz}, 340-3550 \mathrm{~Hz}, 885-23370 \mathrm{~Hz}$ and $1170-5210 \mathrm{~Hz}$ (to collect additional data at the weakest frequencies, the dead band). AMT methods correspond with those used in magnetotelluric (MT) methods (Cagniard, 1953: Vozoff, 1991), but occur at higher frequencies than the lower frequencies of MT . AMT signals originate mainly from atmospheric disturbances and MT signals occur from ionospheric phenomena.

Please, note in the models, that there are gaps in the data at the high frequency end, this is the result of weak signals at some frequencies. Data has not been edited to produce smooth Bostick inversions, but has been edited to eliminate noisy data and repeated data at various frequencies.

\section{Why Use AMT?}

Measurement of earth resistivities can be an effective means of mapping geologic structures because of the large variation in the electrical resistivity of rocks, and in many instances, can be used to determine whether the rocks are intrusive, volcanic, sedimentary, or metamorphic. Alteration and fault zones can also be mapped. The resistivity of geologic units is largely dependent upon their fluid content, porosity, fracturing, temperature, and conductive mineral 
content (Keller and Frischknecht, 1966).

Unaltered, unfractured crystalline rocks are normally very resistive (typically $1,000 \mathrm{ohm}-\mathrm{m}$ or greater), whereas faults will show low resistivity (less tan $100 \mathrm{ohm}-\mathrm{m}$ ) when they are comprised of rocks fractured enough to have hosted fluid transport. Marine sedimentary rocks and clay-rich alluvium are normally very conductive (a few ohm-m to tens of ohm-m). Metamorphic rocks, including gneiss, schist (non-graphitic), and marble are moderately to highly resistive (hundreds to thousands of ohm-m). Tables of electrical resistivity for a variety of rocks, minerals and geological environments may be found in Keller, 1987; Olheoft, 1980; and Palacky, 1987.

\section{References}

Cagniard,L., 1953; Basic theory of the magnetotelluic method: Geophysics, v. 18, no.3, p. 605-635.

EMI, ElectroMagnetic Instruments, Inc., MT-1 Magnetotelluric System Operation Manual, v. 3.2.

Keller, G. V. and Frischknecht, F. C., 1966, Electrical methods in geophysical prospecting: Pergamno Press, New York, $517 \mathrm{p}$.

Keller, G. V., 1987, Rock and mineral properties, in Electromagnetic Methods in Applied Geophysics - Theory: M. N. Nabighian, Ed., Society of Exploration Geophysicists, Tulsa, Oklahoma, vol. 1, p. 13-51.

Olheoft, G. R., 1980, Electrical properties of rocks, in Physical Properties of Rocks and Minerals: Y. S. Touloukian, W. R. Judd, and R. F. Roy, Eds., McGraw-Hill, NewYork, 257330.

Palacky, G. J., 1987, Resistivity characteristics of geologic targets, in Electromagnetic Methods in Applied Geophysics - Theory: M. N. Nabighian, Ed., Society of Exploration Geophysicists, Tulsa, Oklahoma, vol. 1, 53-129.

Vozoff, Keeva, 1991, The magnetotelluric method; in Electromagnetic methods in applied geophysics: Nabighian, M.N., ed., v. 2, Applications, Part B: Tulsa, Society of Exploration Geophysicist, p. 641-711. 


\section{Appendix}

AMT data are presented with nine (9) different plots per station. Bostick inversion for station 1 will be Fig. 1a. The other plots are the Apparent Resistivity, Impedance Phase, Rotation Angle, Impedance Skew, Electrical Multiple Coherency, Tipper Magnitude, Tipper Strike, and Polar Plots proceeding as Figs. 1b, 1c, 1d, etc., respectively.

Fig._a. Bostick inversions. Preliminary 1-dimensional inversion models computed using the apparent resistivity and impedance phase.

Fig._b. Apparent resistivity. The relationship of the time-varying magnetic fields and the electrical fields across frequencies.

Fig._c. Impedance Phase. Represents the ratio of the electric field intensity to magnetic field intensity across frequencies.

Fig._d. Rotation angle. Angle between measurement direction and direction of electrical strike, using only the horizontal sensors in the computation.

Fig._e. Impedance Skew. A measurement of the degree of the multi-dimensional resistivity structure. It should be near 0 for 1-dimensional resistivity structures but larger for 2 or 3dimensional structures, and very large for noisy data.

Fig._f. E Multiple Coherency. A measure of how well the electrical field correlates with the magnetic field. High coherency generally means low noise in the data.

Fig._g. Tipper Magnitude. A measure of nearby vertical resistivity structure (s). Values close to 0 represents horizontal layering.

Fig._h. Tipper Strike, Angle between measurement direction and direction of electrical strike. Uses both the horizontal sensors and the vertical coil to give a less ambiguous rotation.

Fig._i. Polar Plots. Angular variations in impedance. The simpler circular plots often denote 1dimensionality; plots that are peanut-shaped denote 2-dimensionality; plots that are four-leaf clover shaped denote 3 -dimensionality and more complex denote noise at that frequency. 


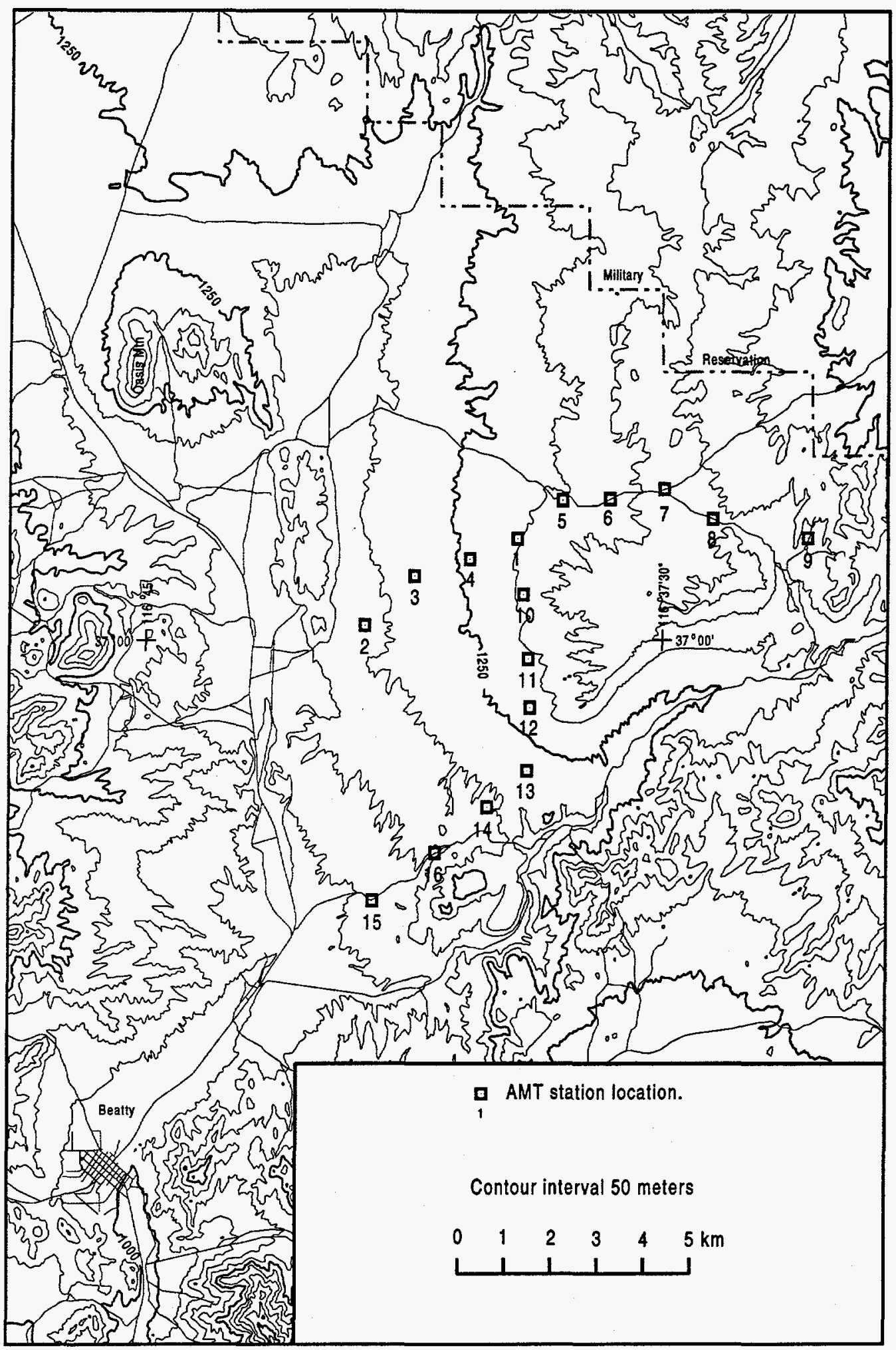

Figure 1. AMT station locations. 
Resistivity (Bostick Inv.)

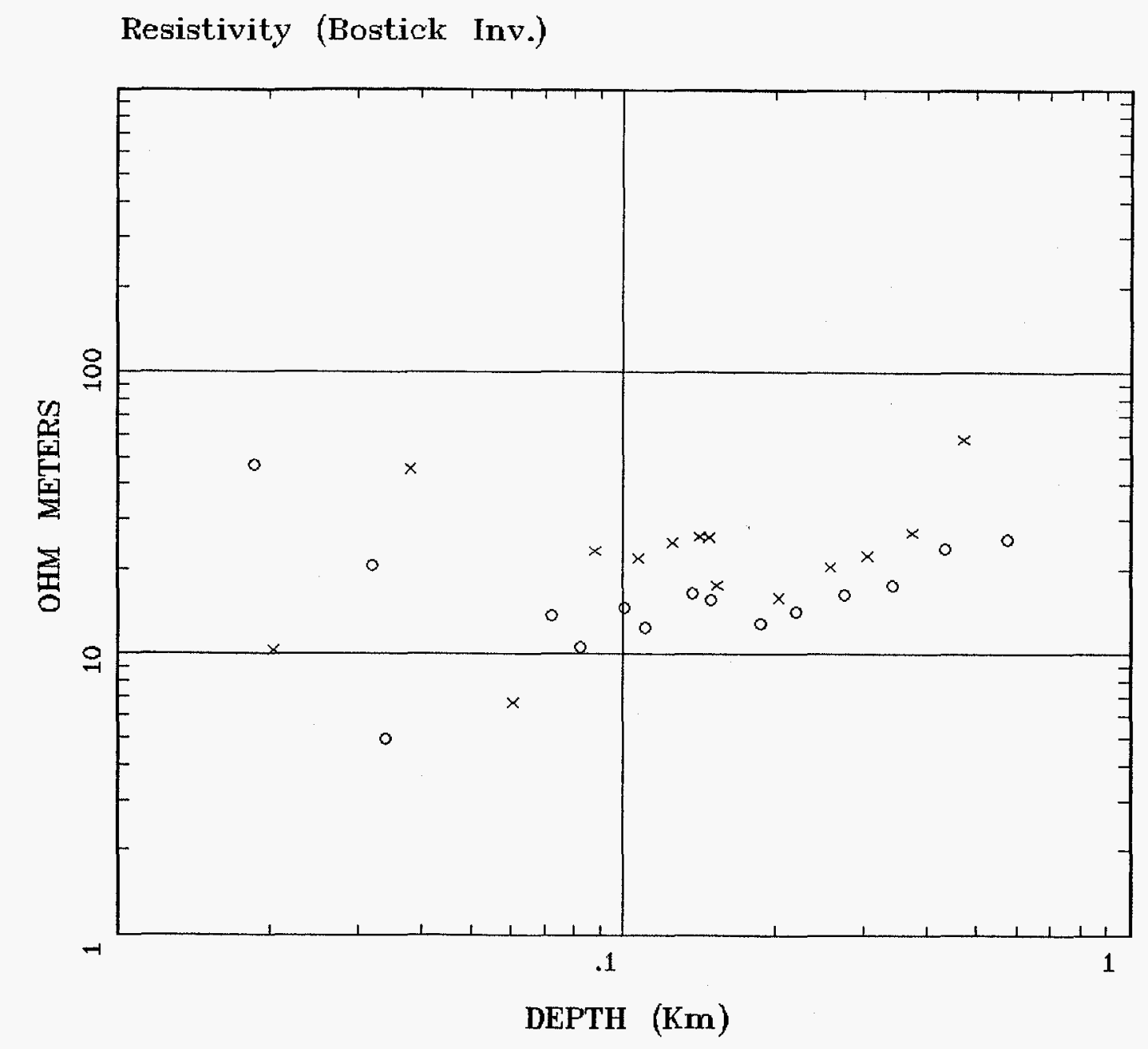

Client:

Remote:

Acquired: $17: 5$ Jun 08, 1997 Survey $\mathrm{Co}$ :
Rotation:

Filename: aba01comb.all

Channels: Ex1 Ey1 Hx1 Hy1 Hz1 Ex2

Plotted: 18:57 Jun 08, 1997

< EMI - ElectroMagnetic Instruments

Fig. $1 \mathbf{a}$ 


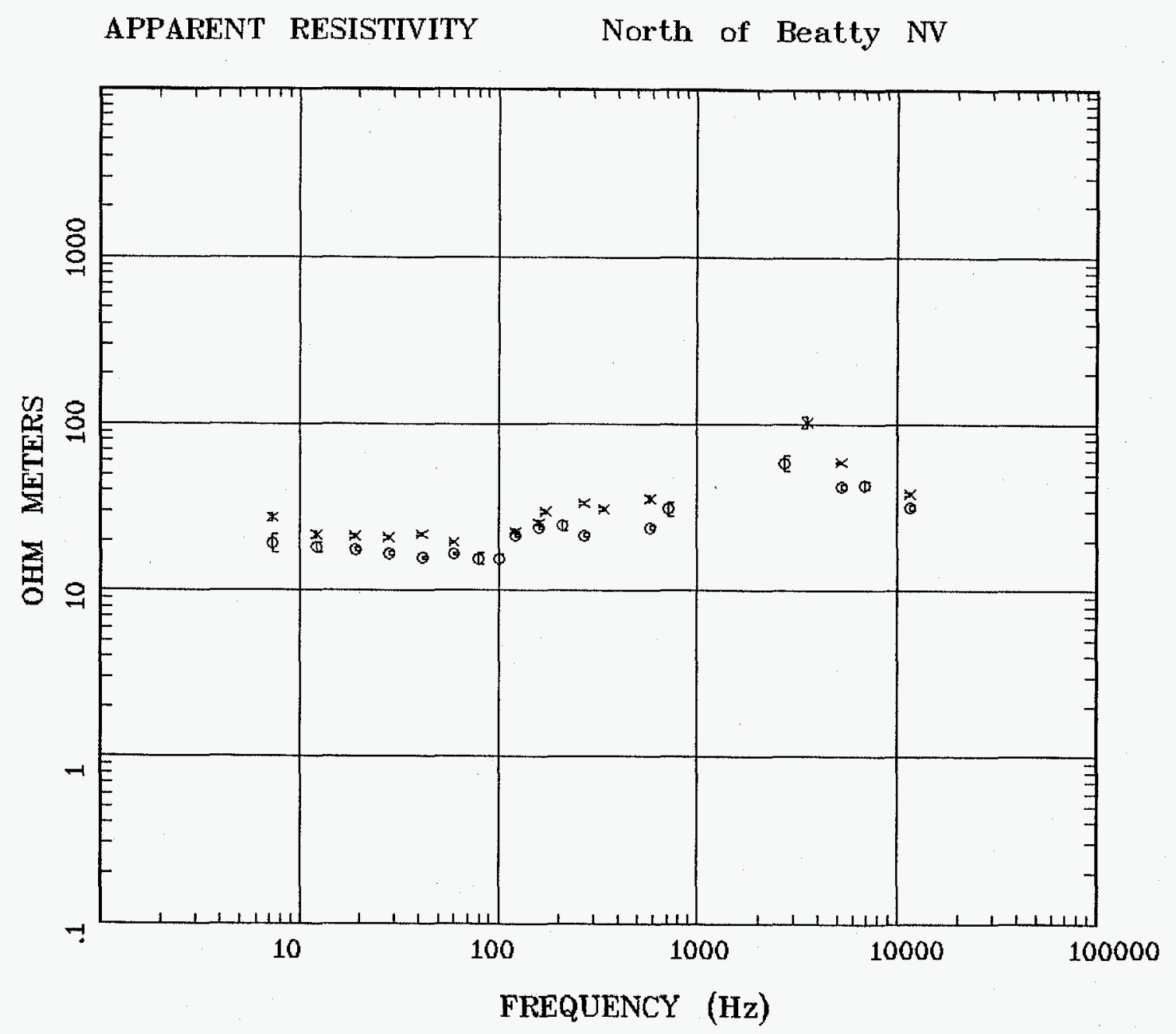

Client:

Remote: remote $\mathrm{e}$

Acquired: 17:5 Jun 08, 1997

Survey Co:USGS
Rotation:

Fileaame: aba01comb.all

Channels: Ch1 Ch2 Ch3 Ch4 Ch5 Ch6

Plotted: 18:57 Jun 08, 1997

< EMI - ElectroMagnetic Instruments

Fig. 1b 

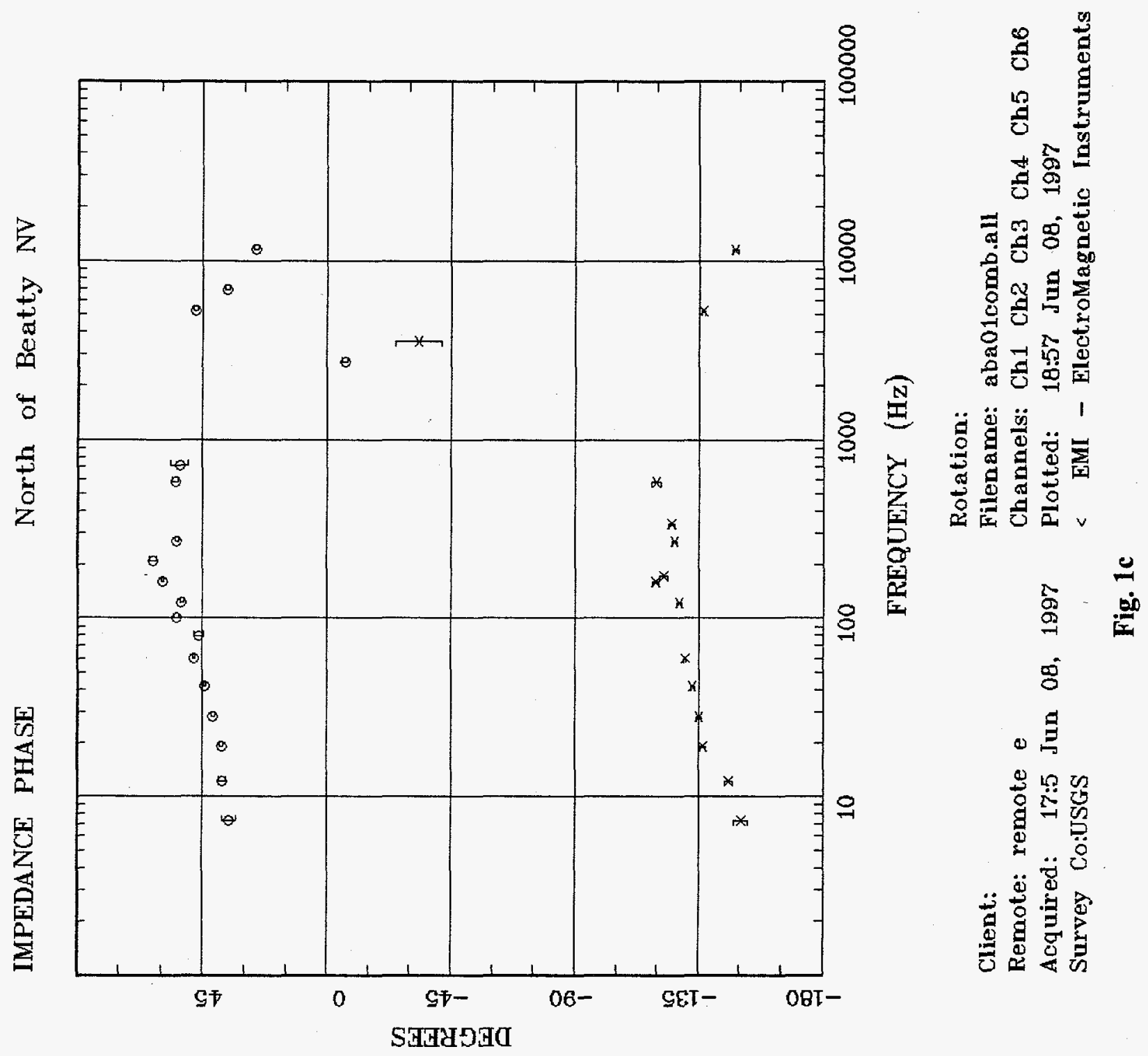
ROTATION ANGLE

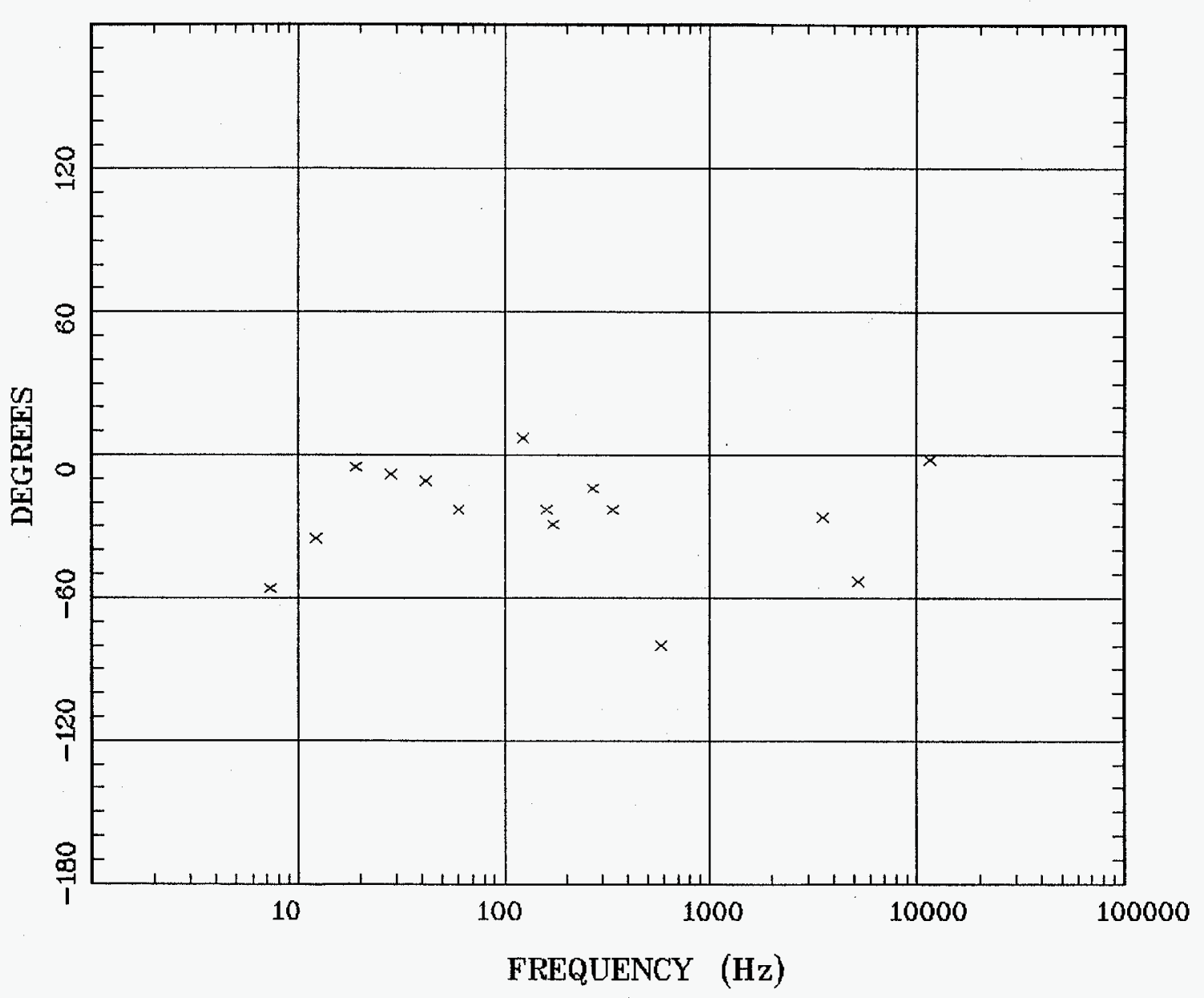

\section{Client:}

Remote: remote e

Acquired: 17:5 Jun 08, 1997 Survey Co:USGS
Rotation:

Filename: aba01comb.all

Channels: Ch1 Ch2 Ch3 Ch4 Ch5 Ch6

Plotted: 18:57 Jun 08, 1997

< EMI - ElectroMagnetic Instruments

Fig. 1d 


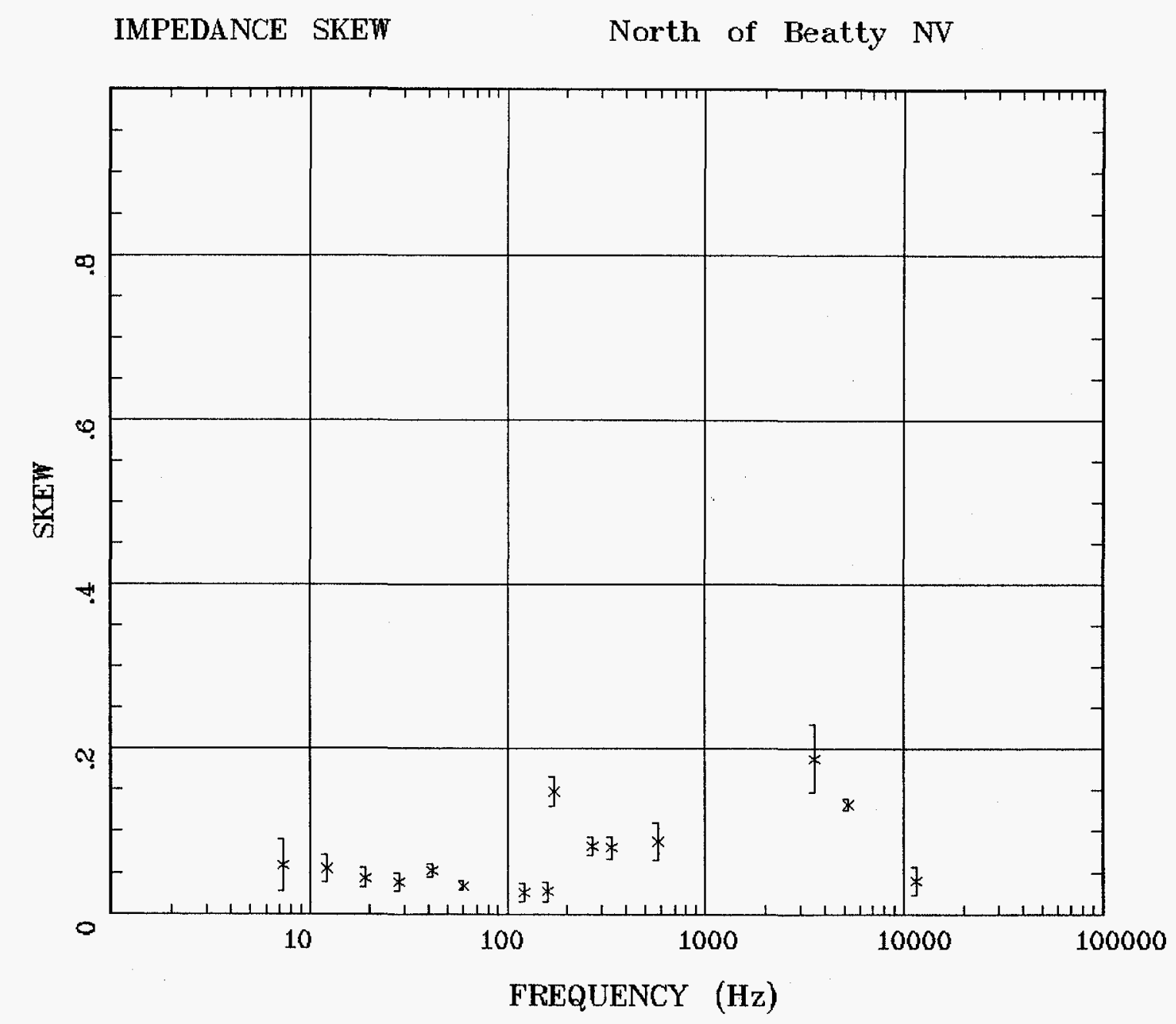

Client:

Remote: remote e

Acquired: 17:5 Iun 08, 1997 Survey Co:USGS

\section{Rotation:}

Filename: aba01comb.all

Channels: Ch1 Ch2 Ch3 Ch4 Ch5 Ch6

Plotted: 18:57 Jun 08, 1997

$<$ EMI - ElectroMagnetic Instruments

Fig. 1e 


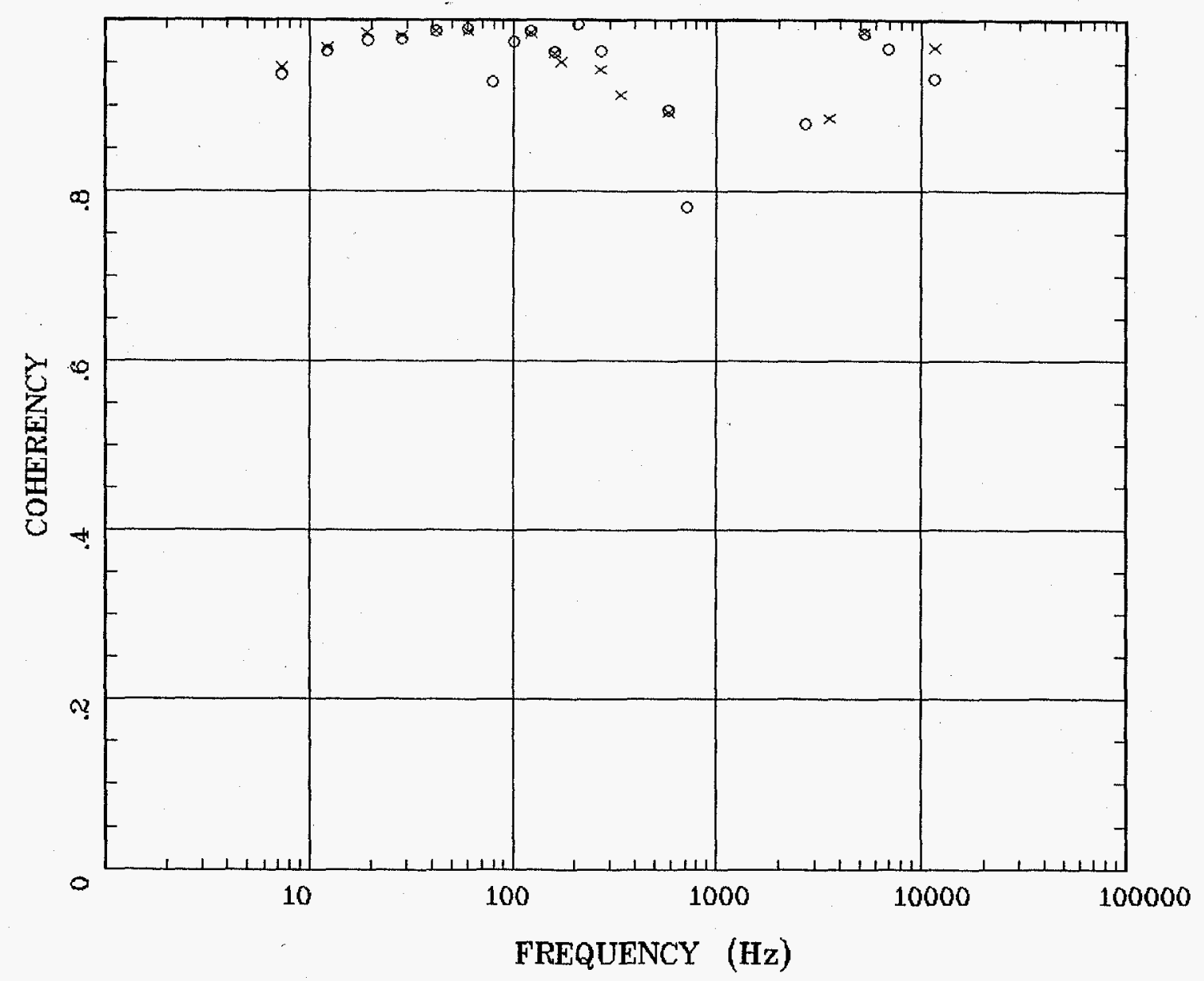

\section{Client:}

Remote: remote $\mathrm{e}$

Acquired: 17:5 Jun 08, 1997 Survey Co:USGS
Rotation:

Filename: aba01comb.all

Channels: Ch1 Ch2 Ch3 Ch4 Ch5 Ch6

Plotted: 18:57 Jun 08, 1997

< EMI - ElectroMagnetic Instruments

Fig. 1f 


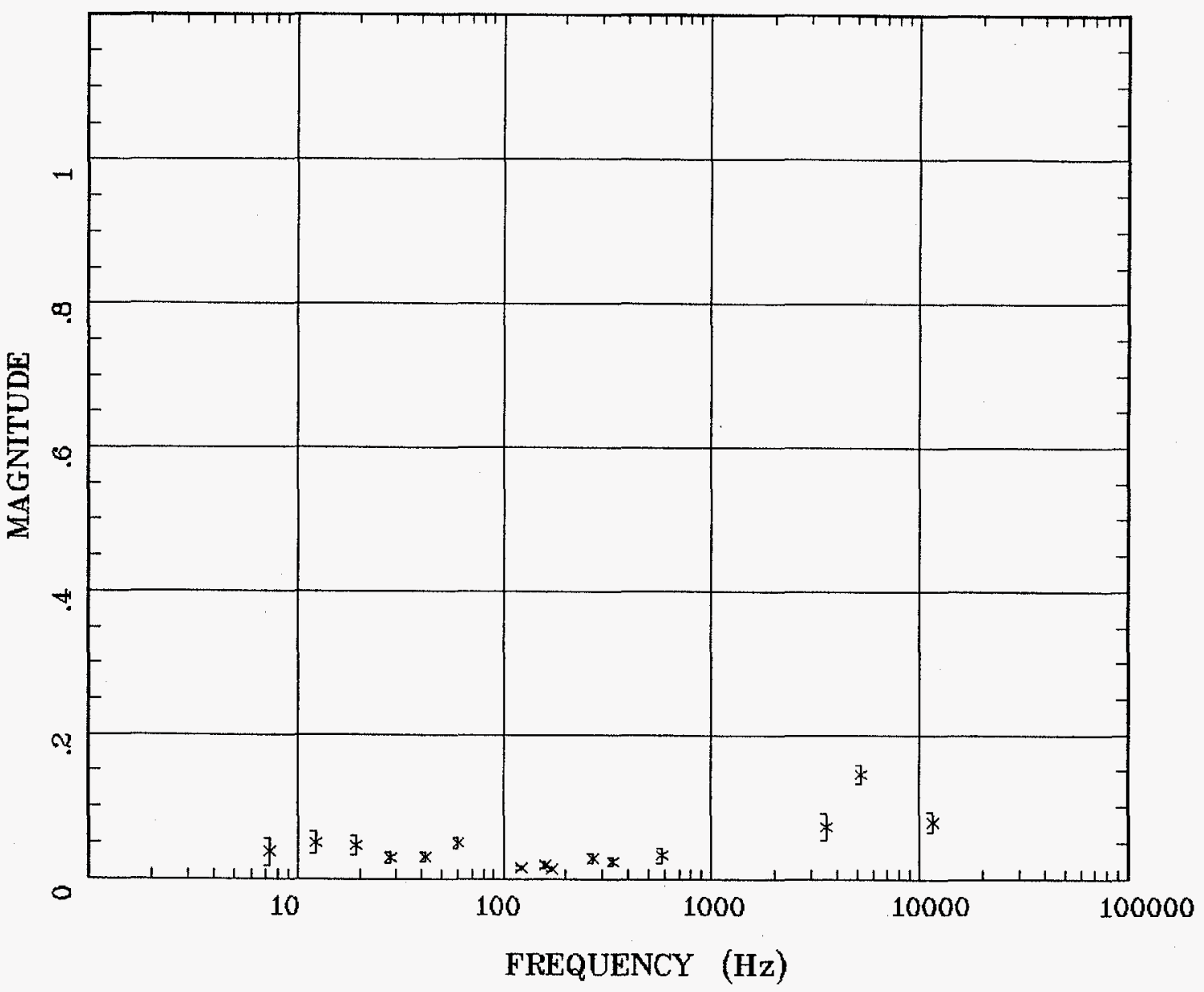

\section{Client:}

\section{Rotation:}

Remote: remote e

Filename: aba01comb.all

Acquired: 17:5 Jun 08, 1997

Survey Co:USGS

Channels: Ch1 Ch2 Ch3 Ch4 Ch5 Ch6

Plotted: 18:58 Jun 08, 1997

$<$ EMI - ElectroMagnetic Instruments

Fig. 19 


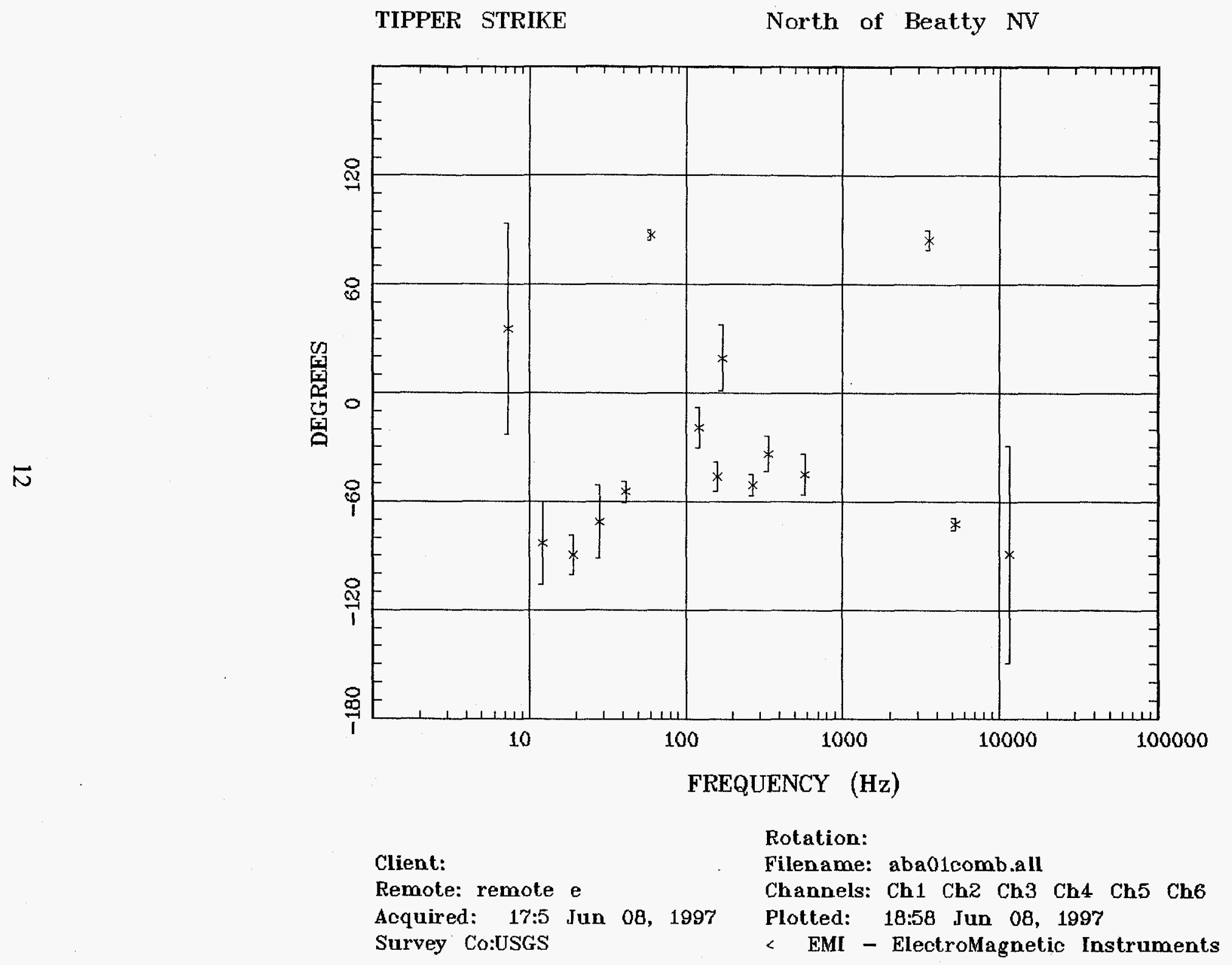

Fig. 1h 


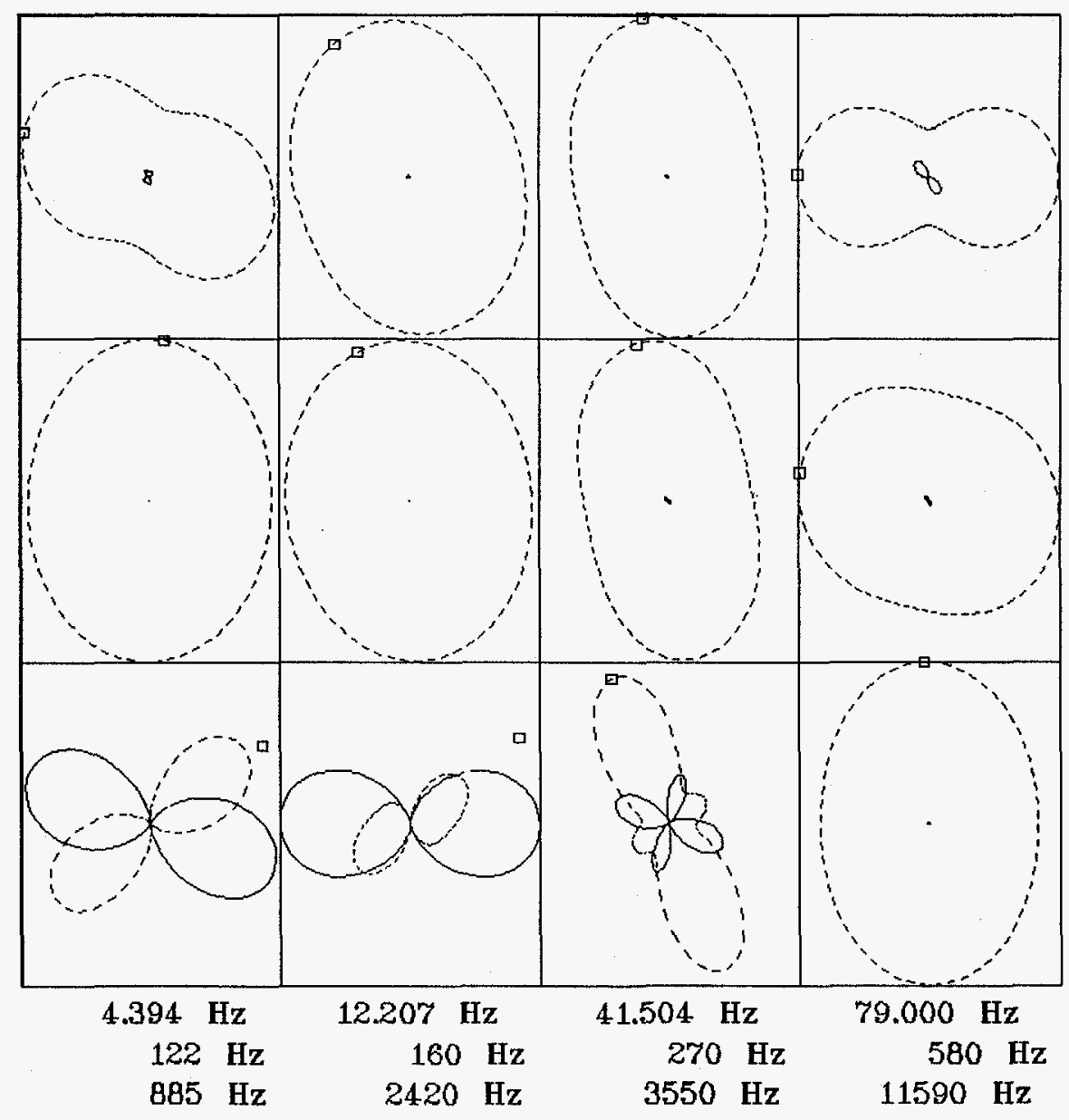

Client:

Rotation:

Remote: remote e Channels: Ch1 Ch2 Ch3 Ch4 Ch5 Ch6 Ch7

Filename: aba01comb.all Acquired: 17:5 Jun 08, 1997 Plotted: 18:58 Jun 08, 1997

Survey Co:USGS

< EMI - ElectroMagnetic Instruments >

Fig. 1i 


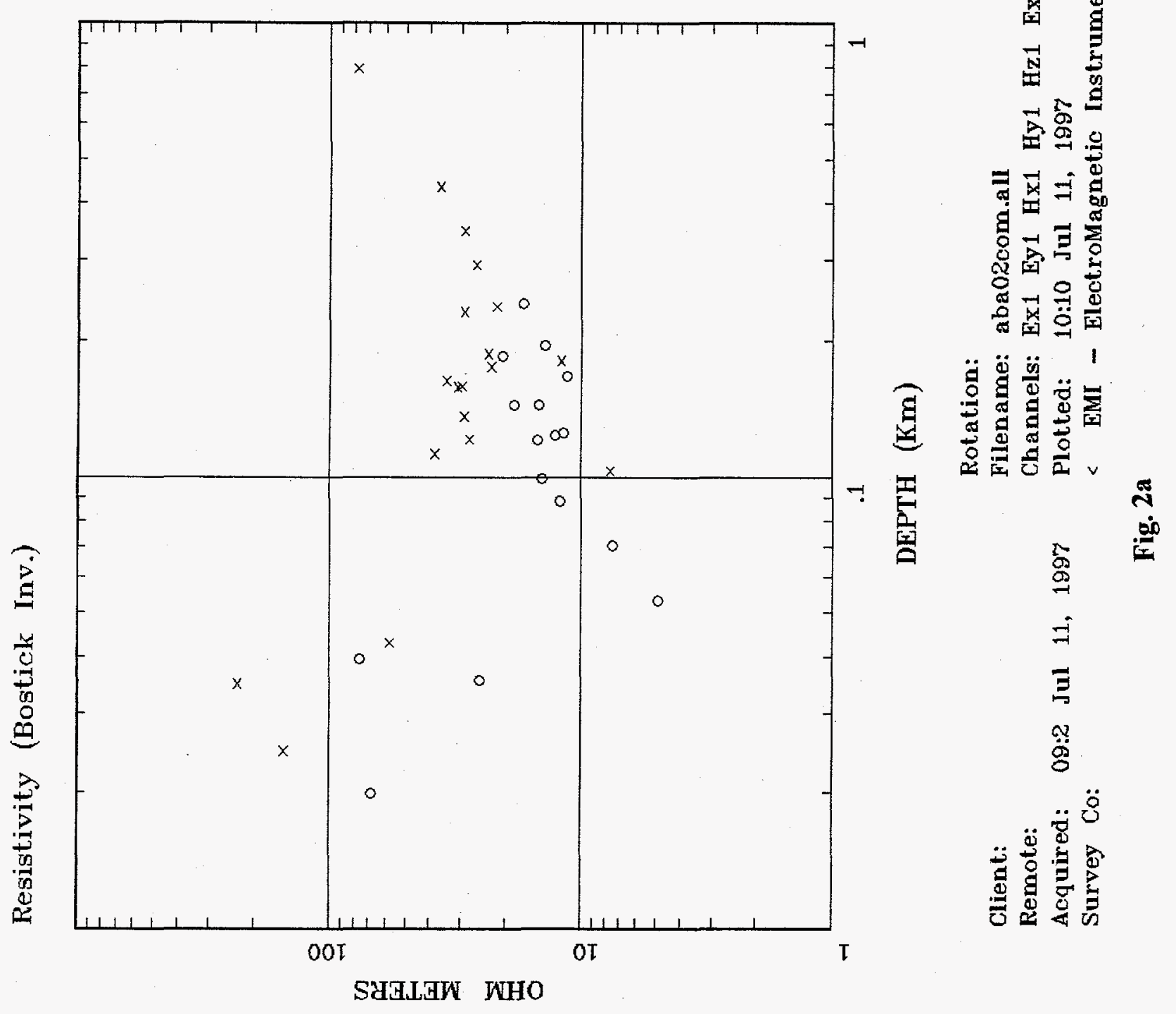




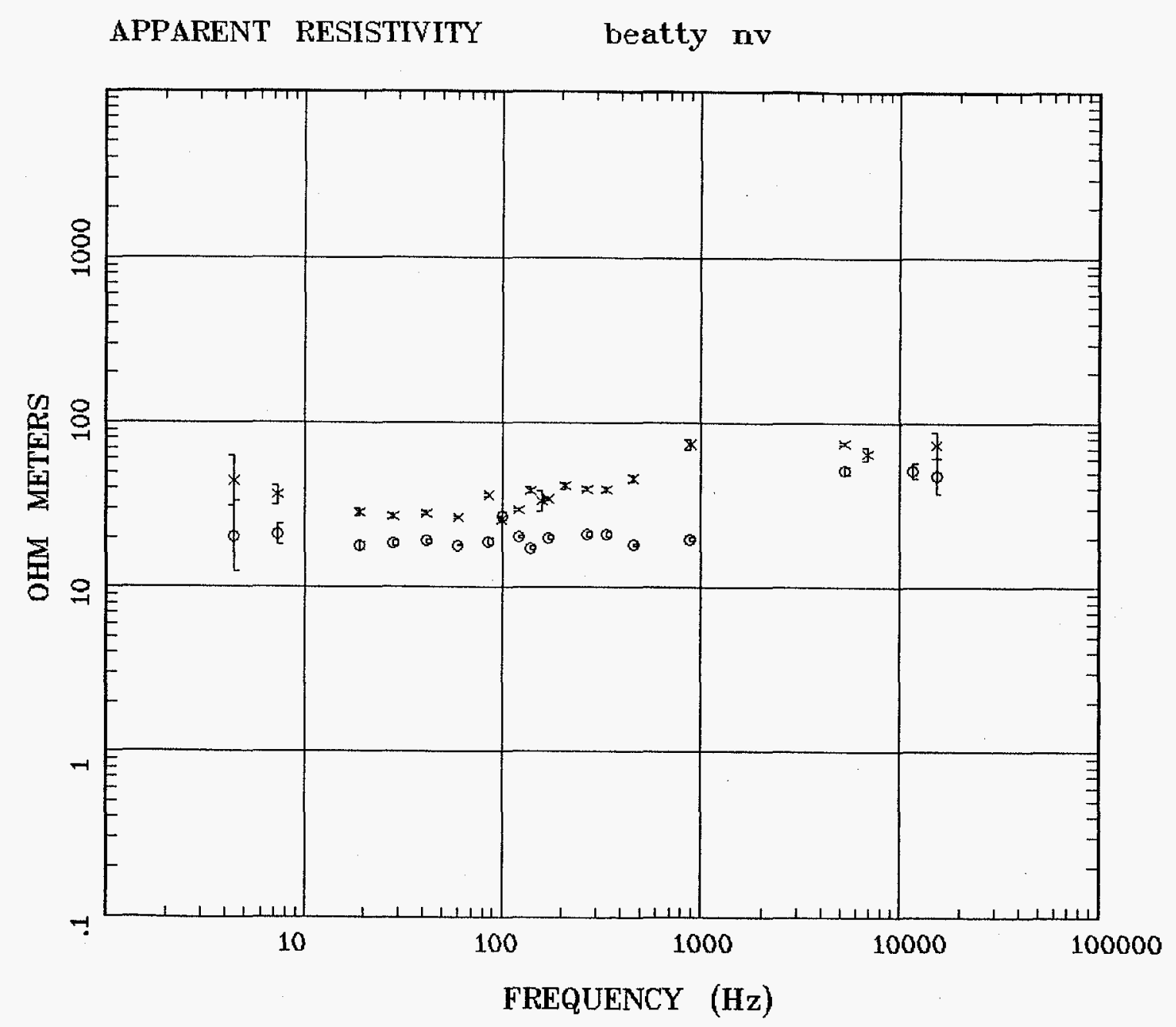

Client:

Remote: remote e

Acquired: 09:2 Jul 11, 1997

Survey Co:usgs
Rotation:

Filename: aba02com.all

Channels: Ch1 Ch2 Ch3 Ch4 Ch5 Ch6

Plotted: 10:10 Jul 11, 1997

< EMI - ElectroMagnetic Instruments

Fig. 2b 
IMPEDANCE PHASE

beatty $\mathrm{nv}$

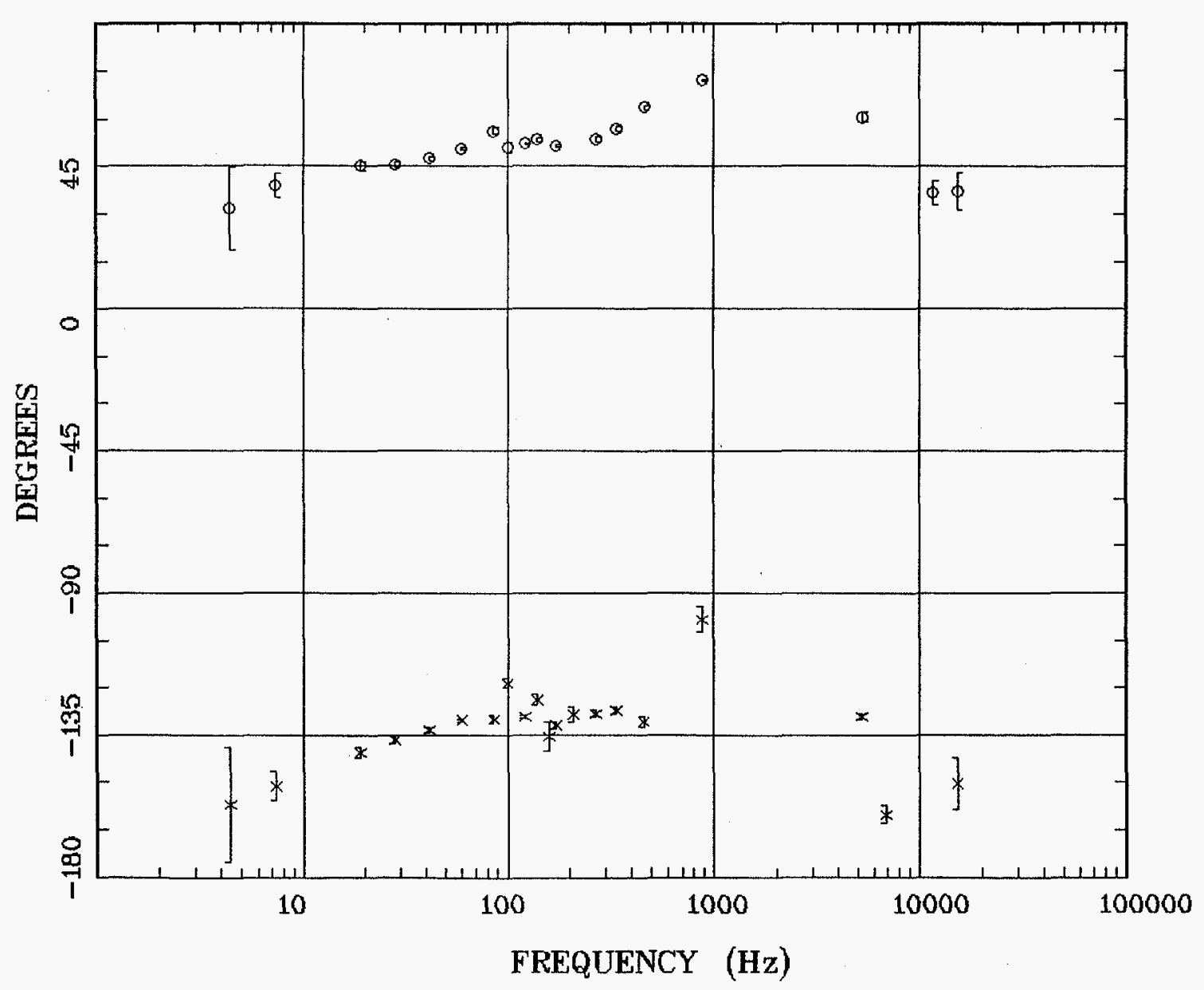

Client:

Remote: remote e

Acquired: 09:2 Jul 11, 1997

Survey Co:usgs

\section{Rotation:}

Filename: aba02comall

Channels: Ch1 Ch2 Ch3 Ch4 Ch5 Ch6 Plotted: 10:11 Jul 11, 1997

< EMI - ElectroMagnetic Instruments

Fig. 2c 
ROTATION ANGLE

beatty $\mathrm{nv}$

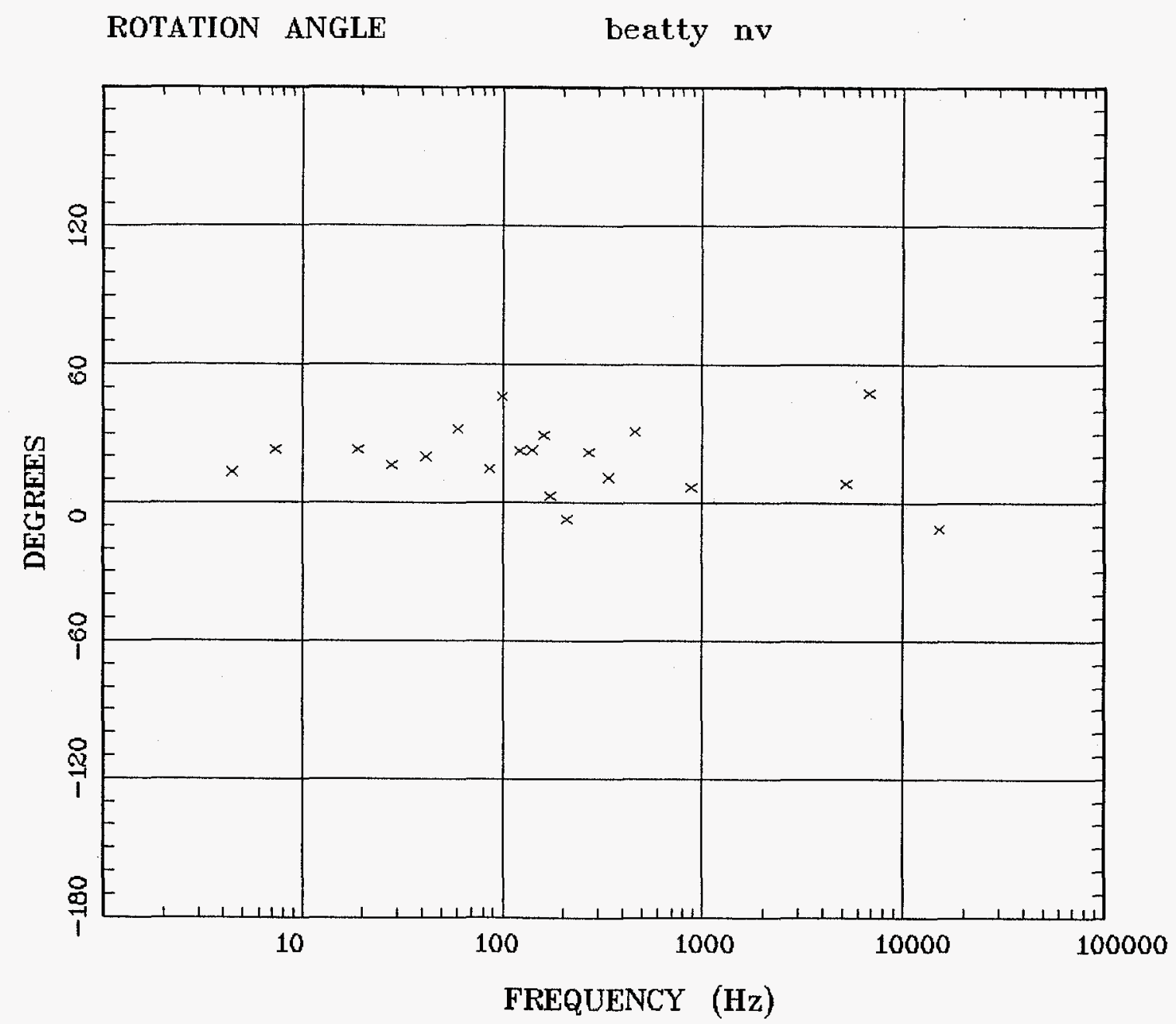

Client:

Remote: remote $\mathrm{e}$

Acquired: 09:2 Jul 11, 1997

Survey Co:usgs
Rotation:

Filename: aba02com.all

Channels: Ch1 Ch2 Ch3 Ch4 Ch5 Ch6

Plotted: $10: 11$ Jul 11, 1997

< EMI - ElectroMagnetic Instruments

Fig. 2d 


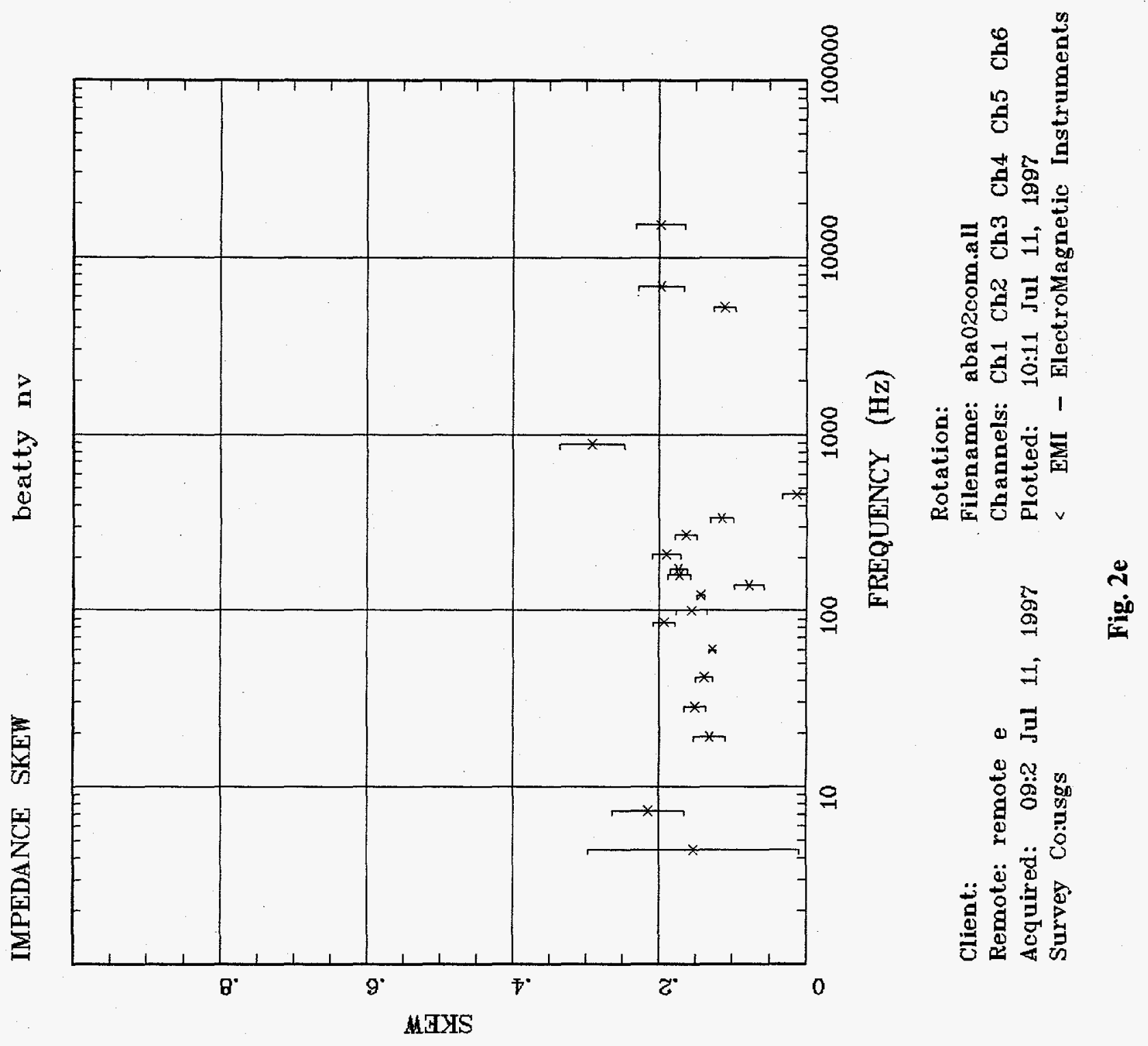


E MULT Coh. beatty nv

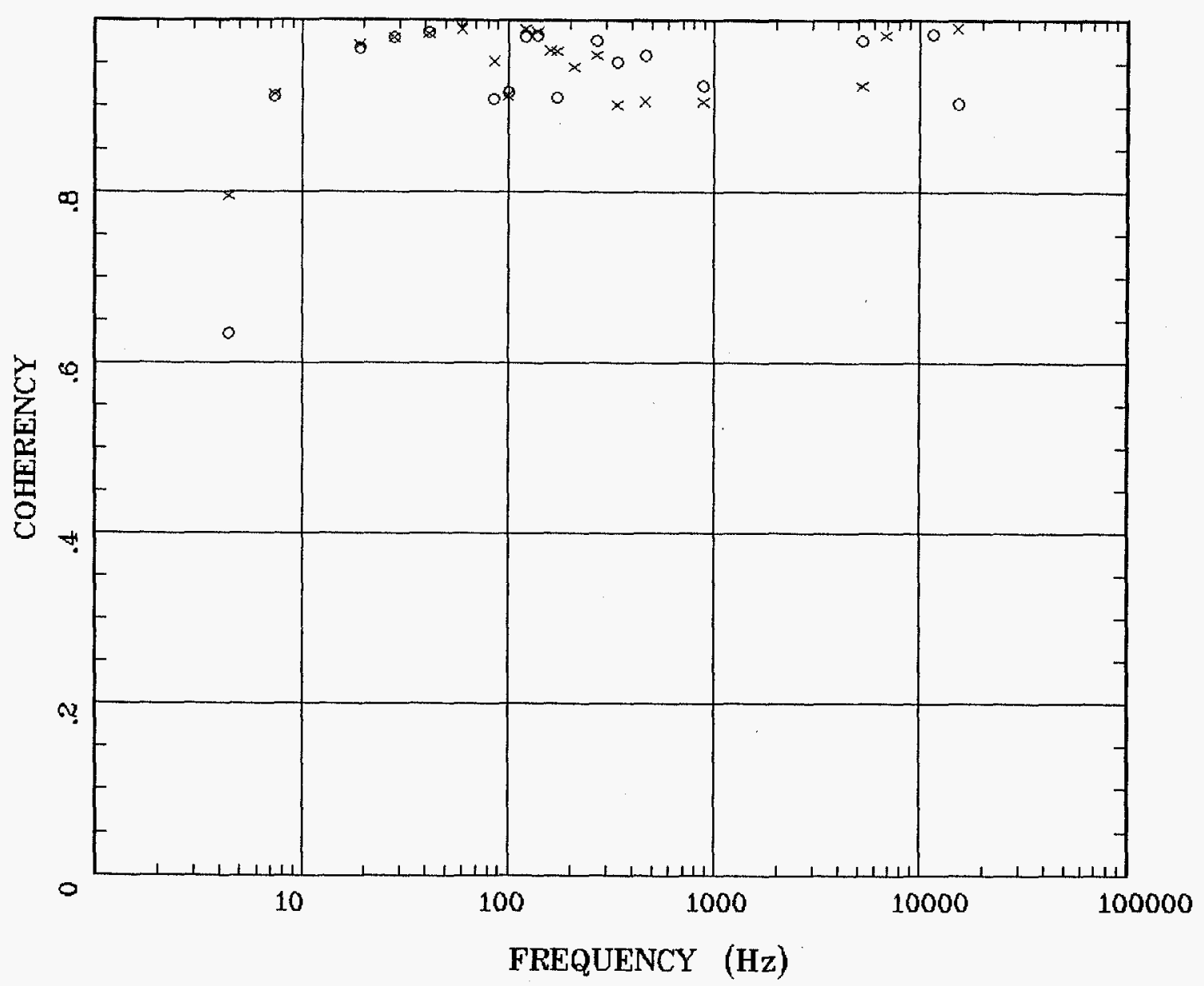

\section{Client:}

Remote: remote e

Acquired: $09: 2$ Jul 11, 1997 Survey Co:usgs
Rotation:

Filename: aba02com.all

Channels: Ch1 Ch2 Ch3 Ch4 Ch5 Ch6

Plotted: 10:11 Jul 11, 1997

< EMI - ElectroMagnetic Instruments

Fig. $2 f$ 


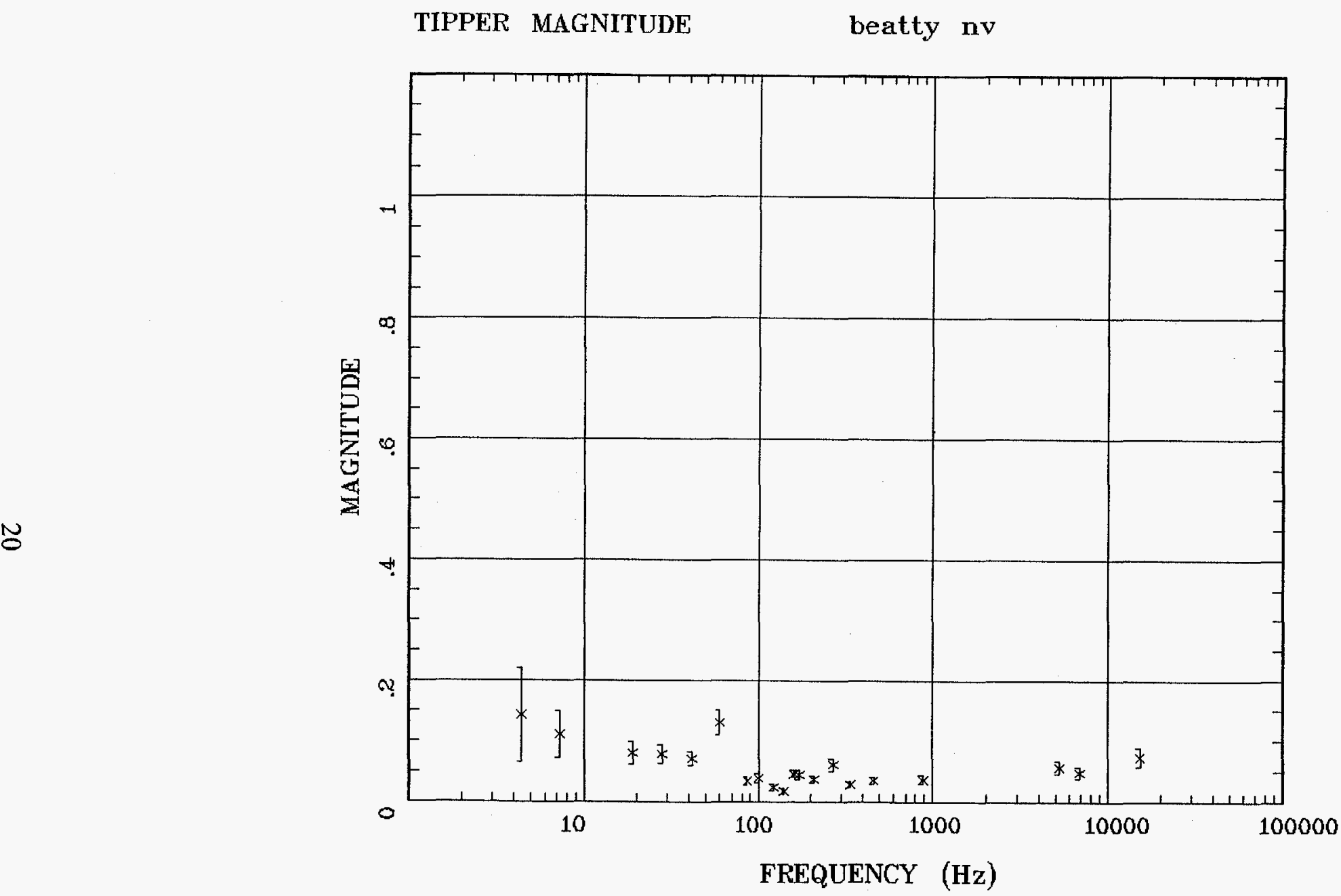

Client:

Remote: remote e

Acquired: 09:2 Jul 11, 1997

Survey Co:usgs
Rotation:

Filename: aba02com.all

Channels: Ch1 Ch2 Ch3 Ch4 Ch5 Ch6

Plotted: 10:11 Jul 11, 1997

$<$ EMI - ElectroMagnetic Instruments

Fig. $2 \mathrm{~g}$ 


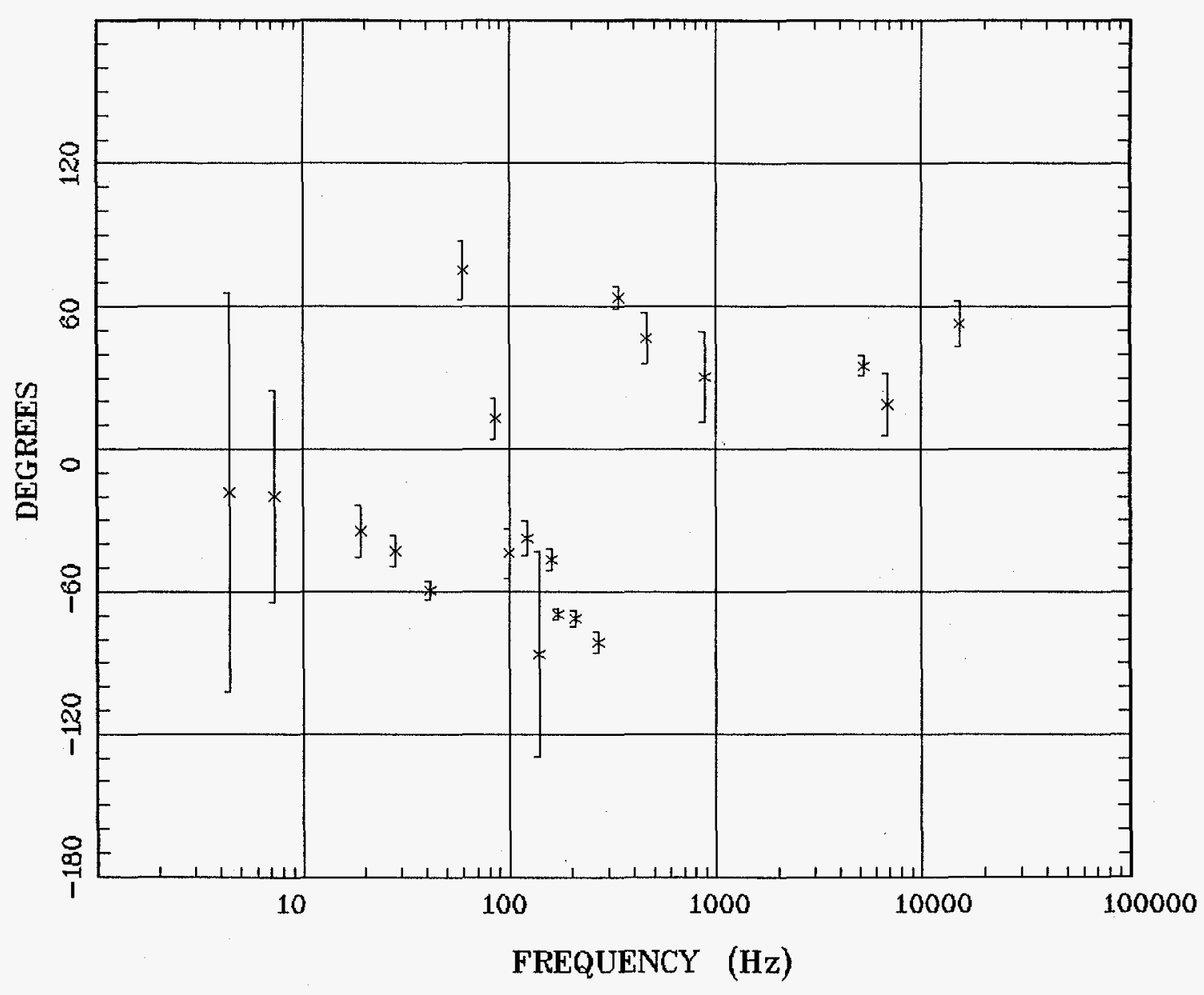

Client:

Remote: remote e Acquired: 09:2 Jul 11, 1997 Survey Co:usgs

\section{Rotation:}

Filename: aba02com.all

Channels: Ch1 Ch2 Ch3 Ch4 Ch5 Ch6 Plotted: 10:11 Jul 11, 1997

< EMI - ElectroMagnetic Instruments

Fig. 2h 


\section{POLAR PLOTS beatty nv}

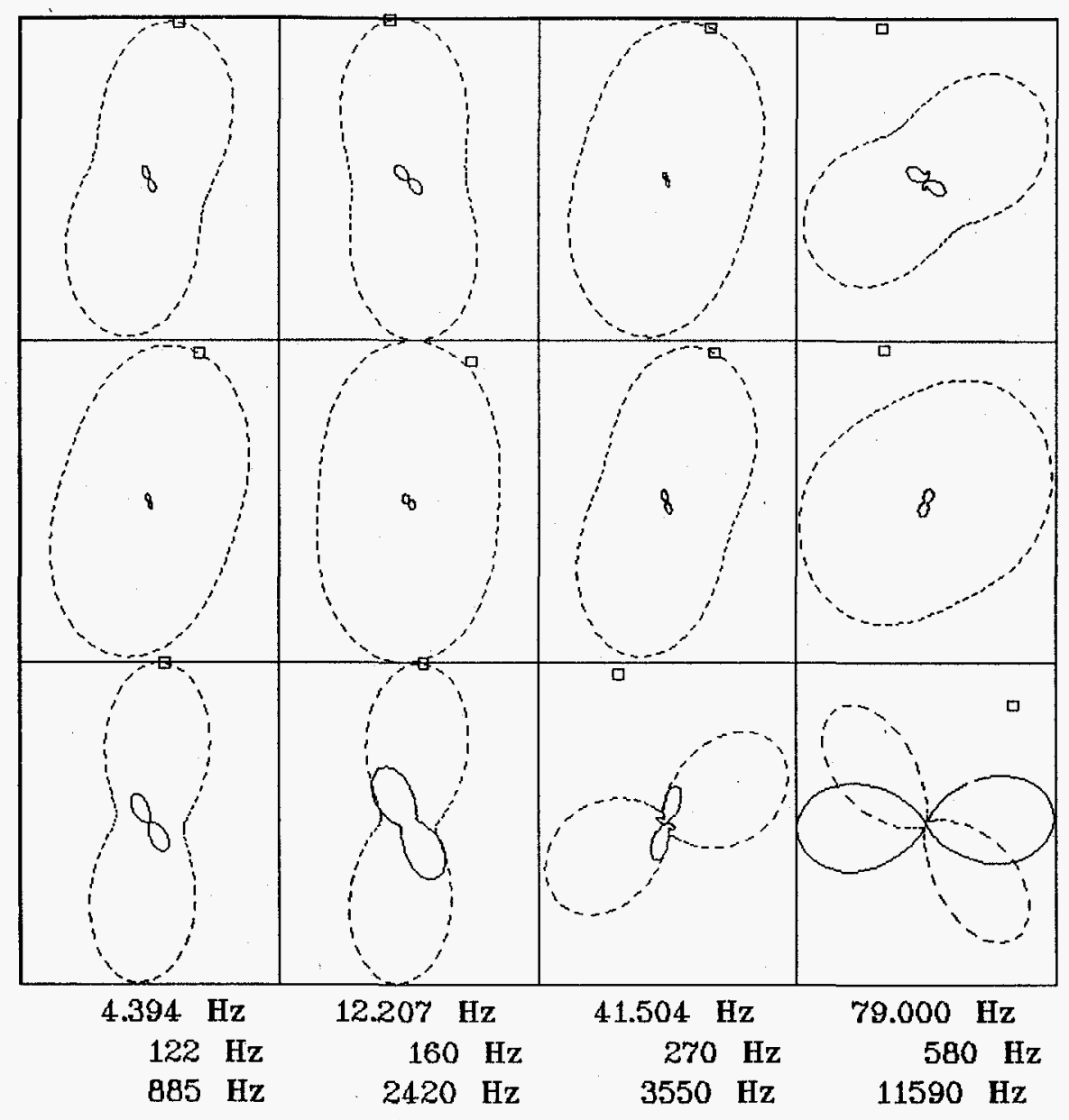

\section{Client:}

Remote: remote e

Acquired: 09:2 Jul

Survey Co:usgs

Rotation:

Filename: aba02comall

Channels: Ch1 Ch2 Ch3 Ch4 Ch5 Ch6 Ch7

Plotted: 10:11 Jul 11, 1997

$<$ EMI - ElectroMagnetic Instruments >

Fig. 2i 


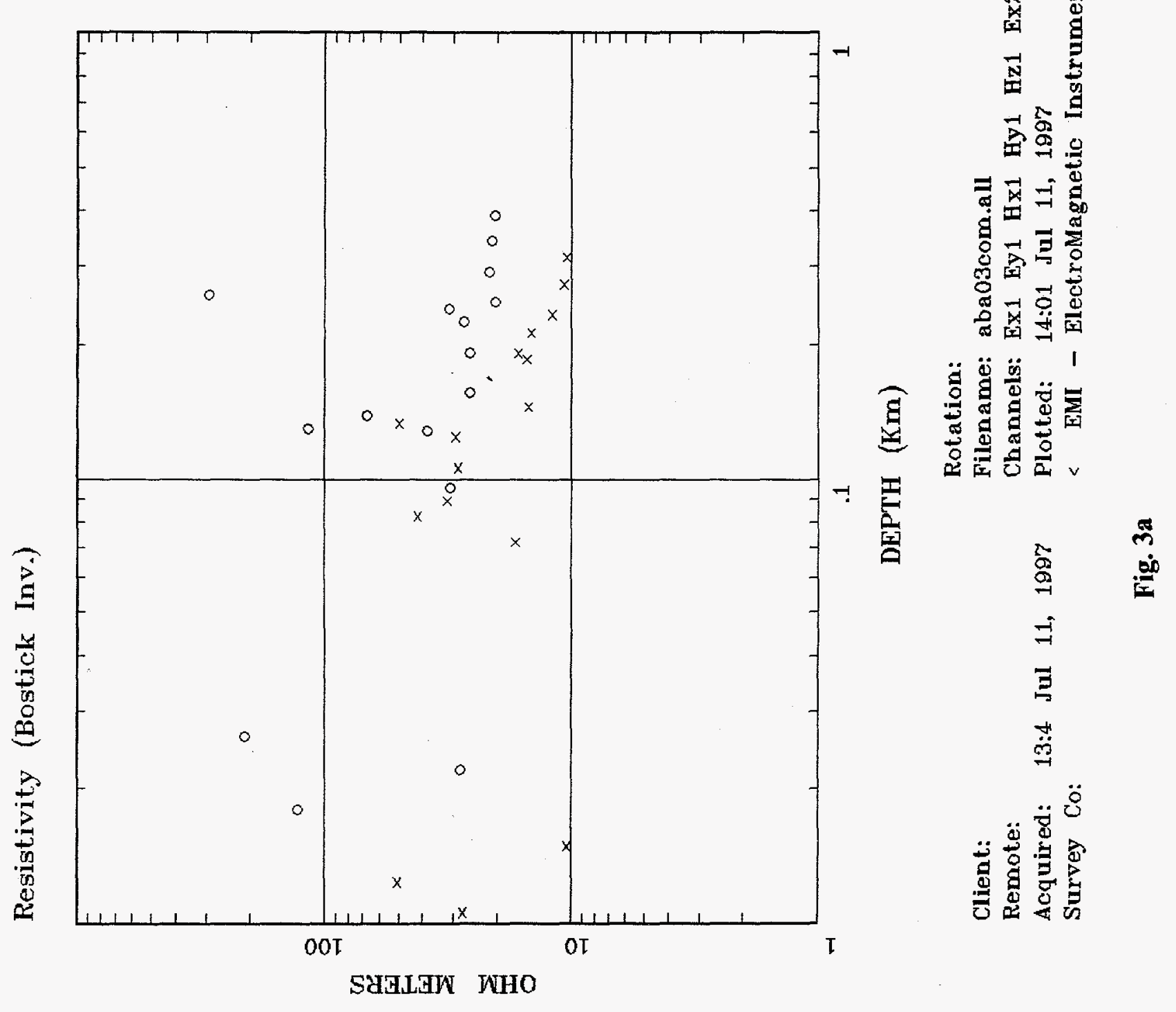




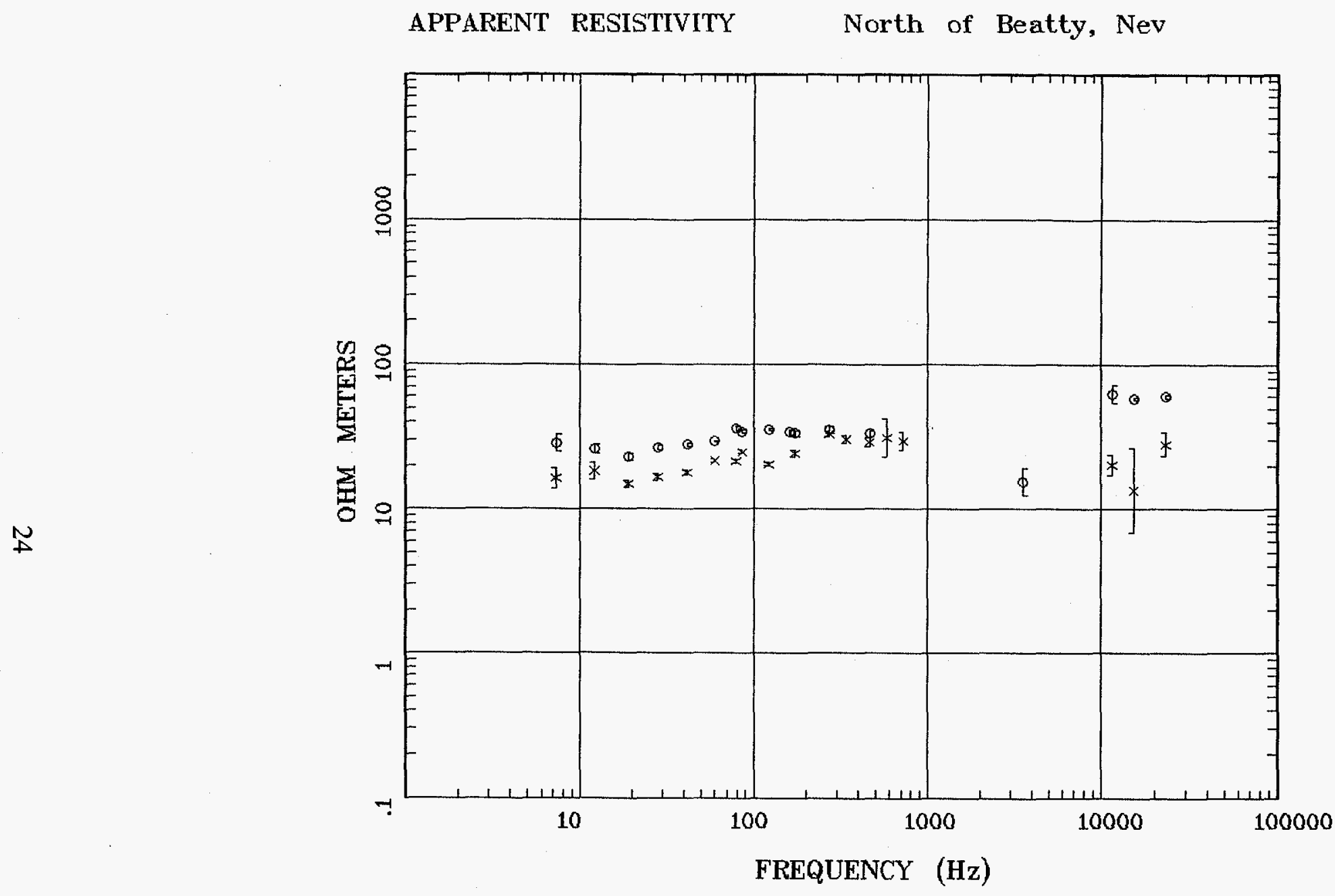

North of Beatty, Nev Client: $\mathbf{n}$

Remote: remote e Acquired: 13:4 Jul 11, 1997 Survey Co:USGS

\section{Rotation:}

Filename: aba03com.all

Channels: Ch1 Ch2 Ch3 Ch4 Ch5 Ch6 Plotted: 14:01 Jul 11, 1997

< EMI - ElectroMagnetic Instruments

Fig. 3b 



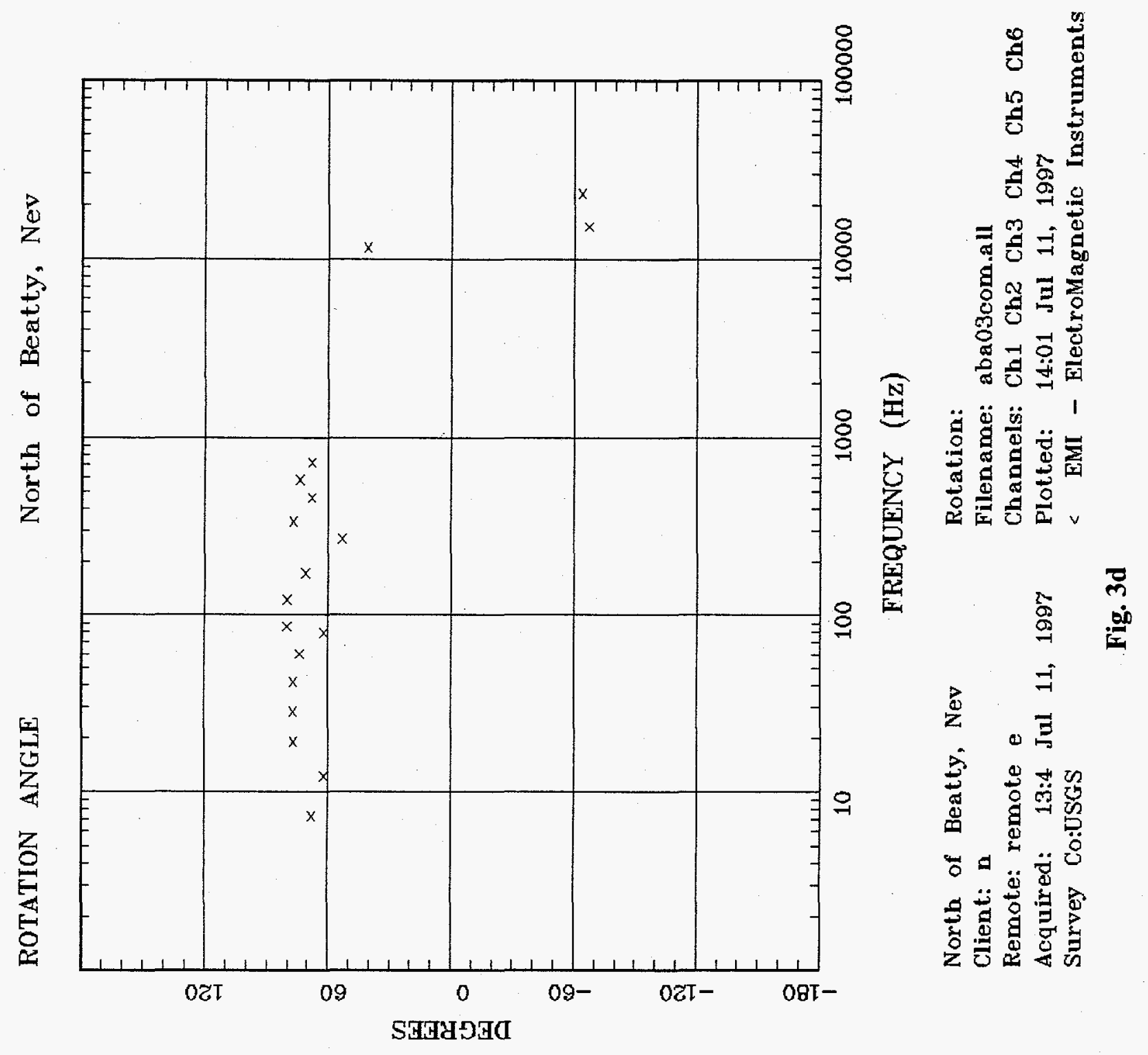




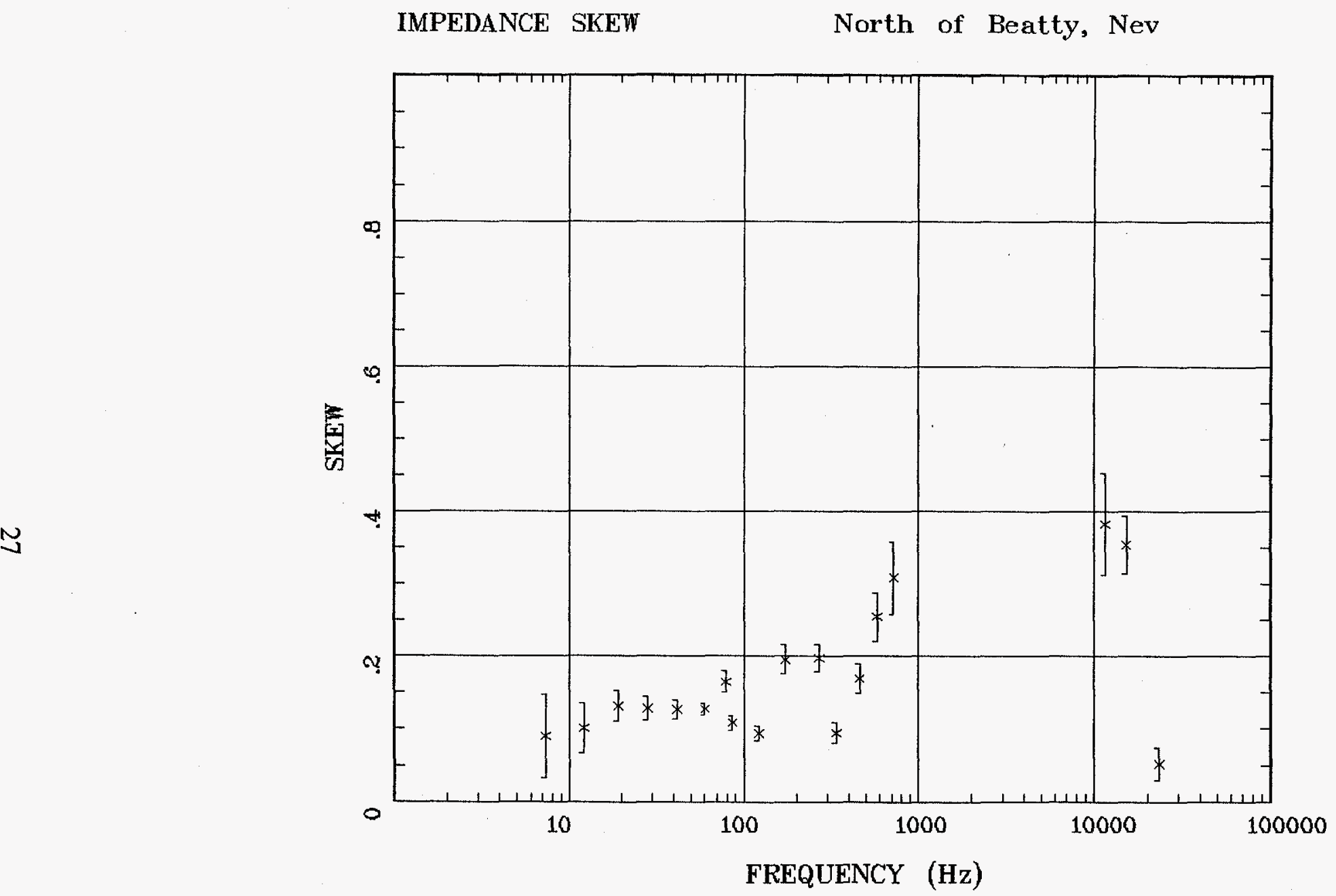

North of Beatty, Nev

Client: $n$

Remote: remote e

Acquired: 13:4 Jul 11, 1997

Survey Co:USGS
Rotation:

Filename: aba03com.all

Channels: Ch1 Ch2 Ch3 Ch4 Ch5 Ch6

Plotted: 14:01 Jul 11, 1997

< EMI - ElectroMagnetic Instruments

Fig. 3e 
North of Beatty, Nev

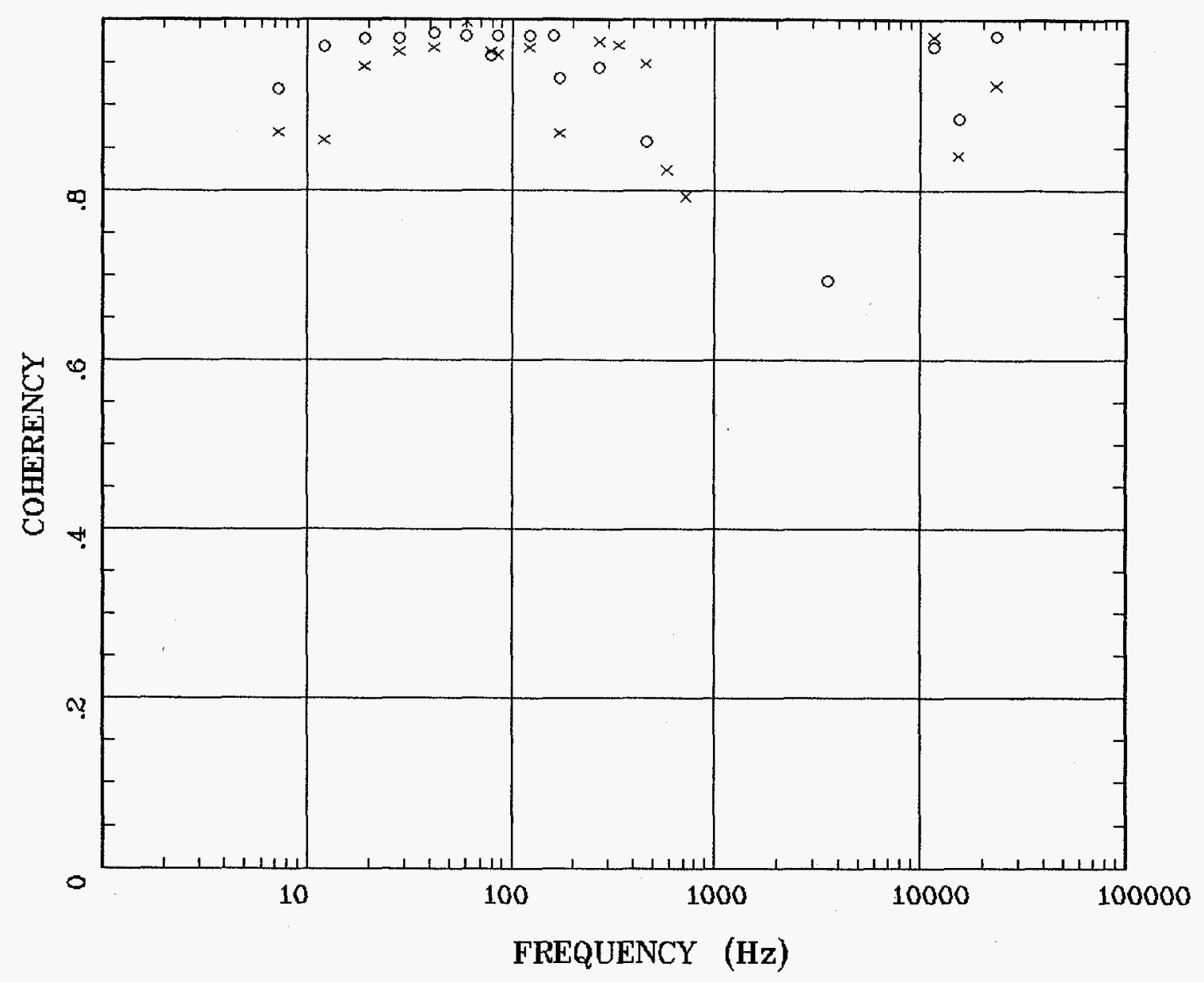

North of Beatty, Nev

Rotation:

Client: $\mathbf{n}$

Filename: aba03com.all

Remote: remote e

Acquired: 13:4 Jul 11, 1997

Channels: Ch1 Ch2 Ch3 Ch4 Ch5 Ch6

Survey Co:USGS

Plotted: 14:01 Jul 11, 1997

< EMI - ElectroMagnetic Instruments

Fig. 3f 
TIPPER MAGNITUDE

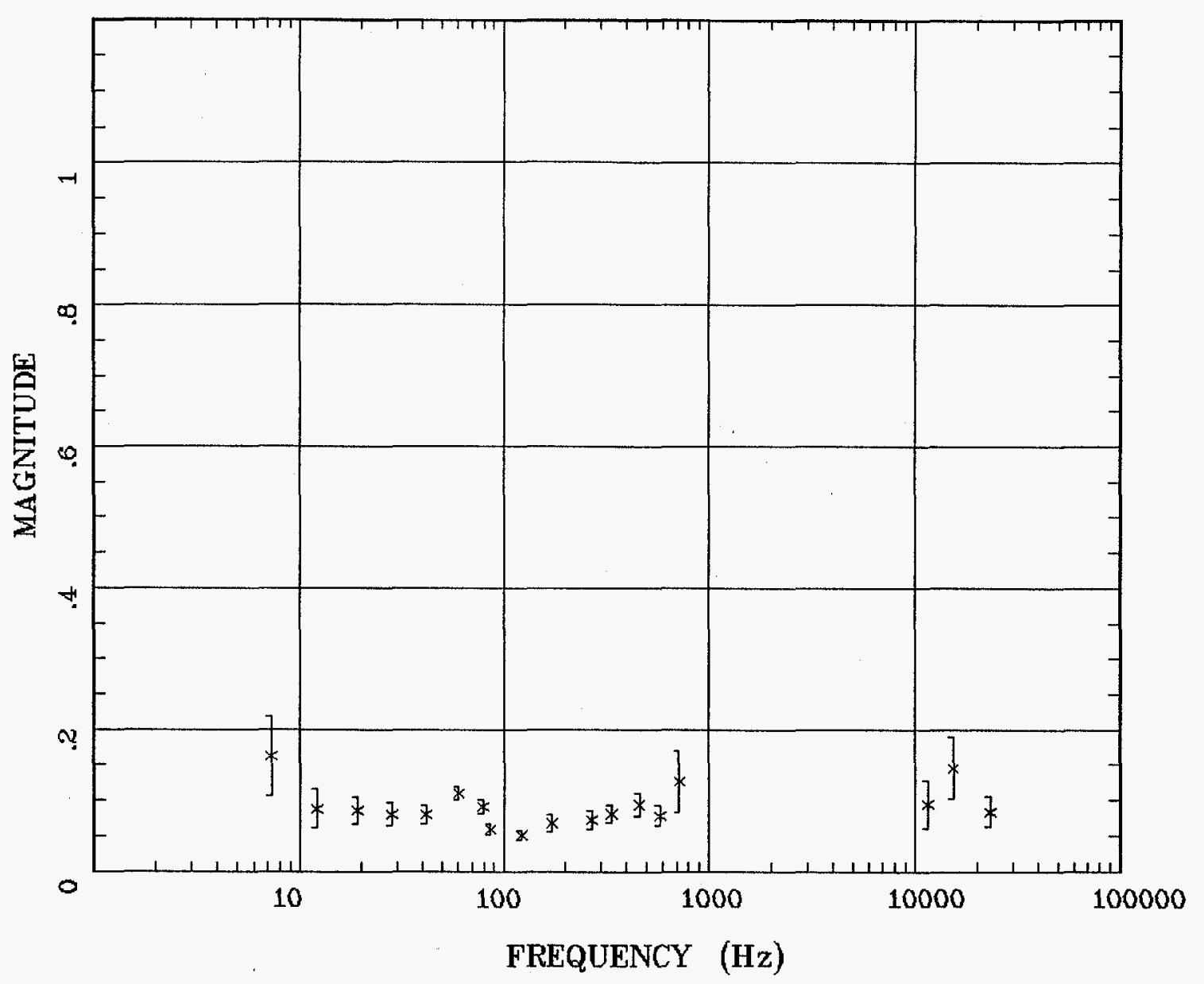

North of Heatty, Nev

Client: $n$

Remote: remote $\mathrm{e}$

Acquired: $13: 4$ Jul 11, 1997

Survey Co:USGS
Rotation:

Filename: aba03com.all

Channels: Ch1 Ch2 Ch3 ch4 ch5 ch6

Plotted: 14:01 Jul 11, 1997

< EMI - ElectroMagnetic Instruments

Fig. 3g 



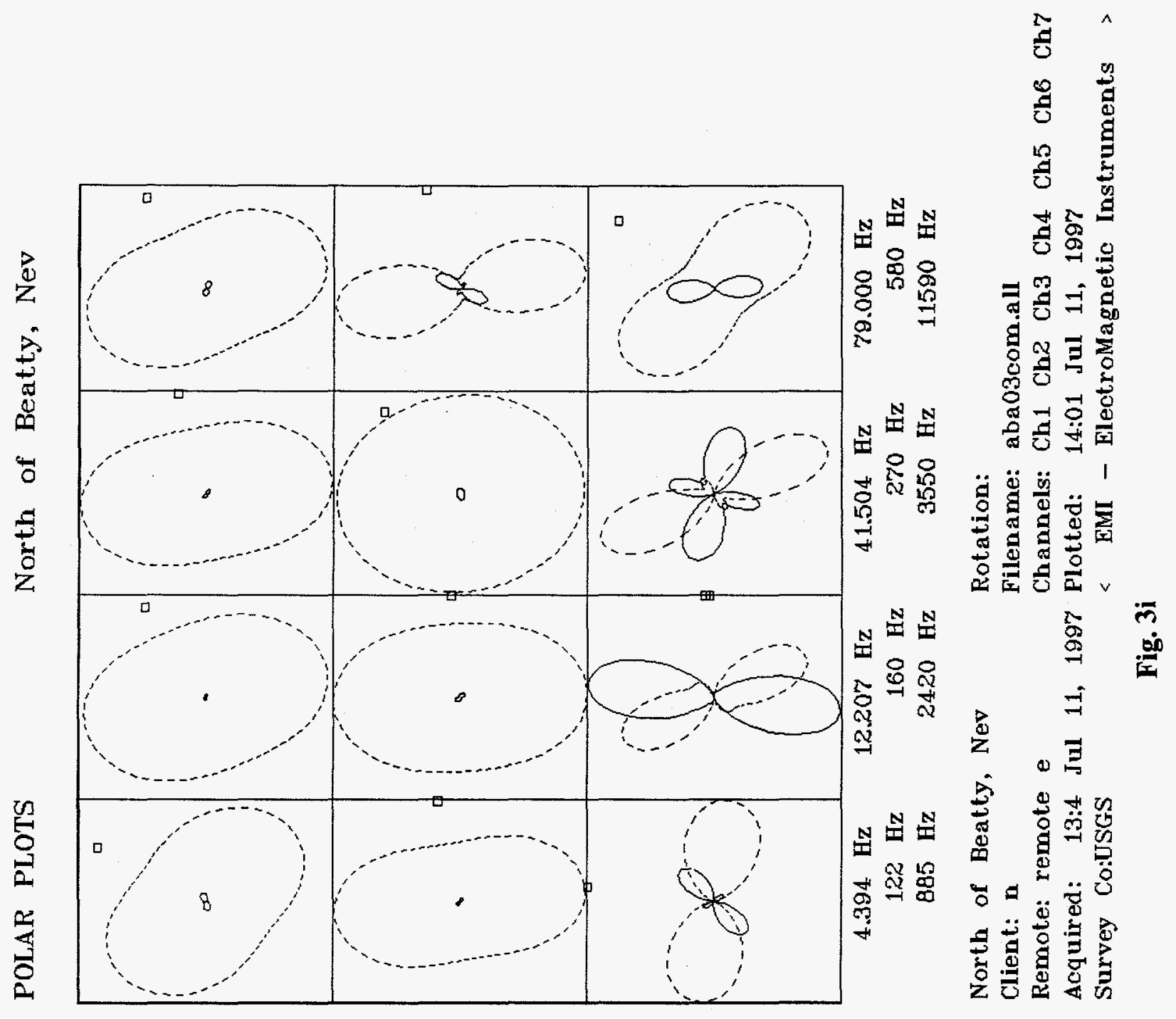




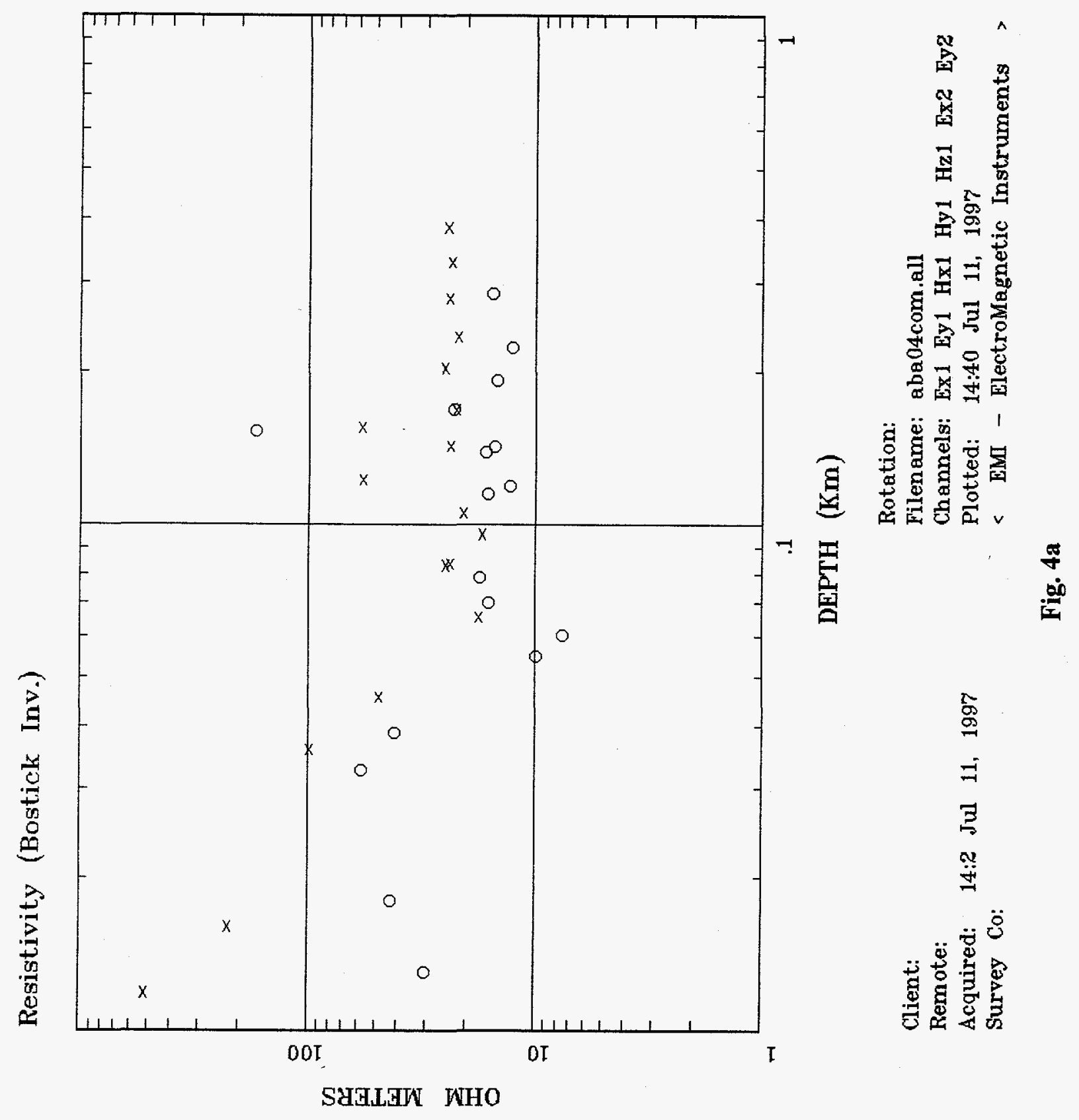




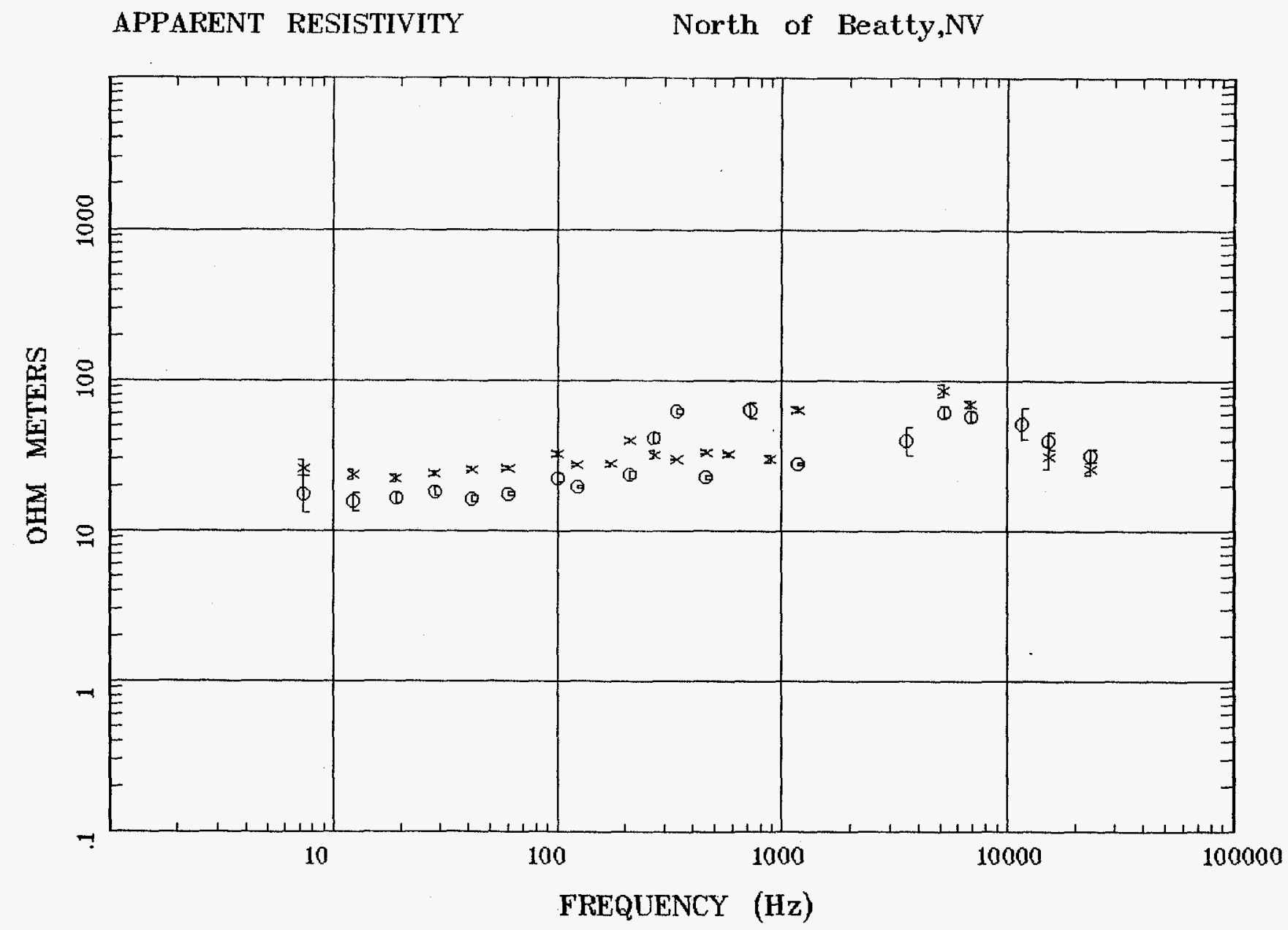

Client:

Remote: remote e

Acquired: 14:2 Jul 11, 1997 Survey Co:USGS
Rotation:

Filename: aba04com.all

Channels: Ch1 Ch2 Ch3 Ch4 Ch5 Ch6 Ch7

Plotted: 14:40 Jul 11, 1997

< EMI - ElectroMagnetic Instruments >

Fig. 4b 


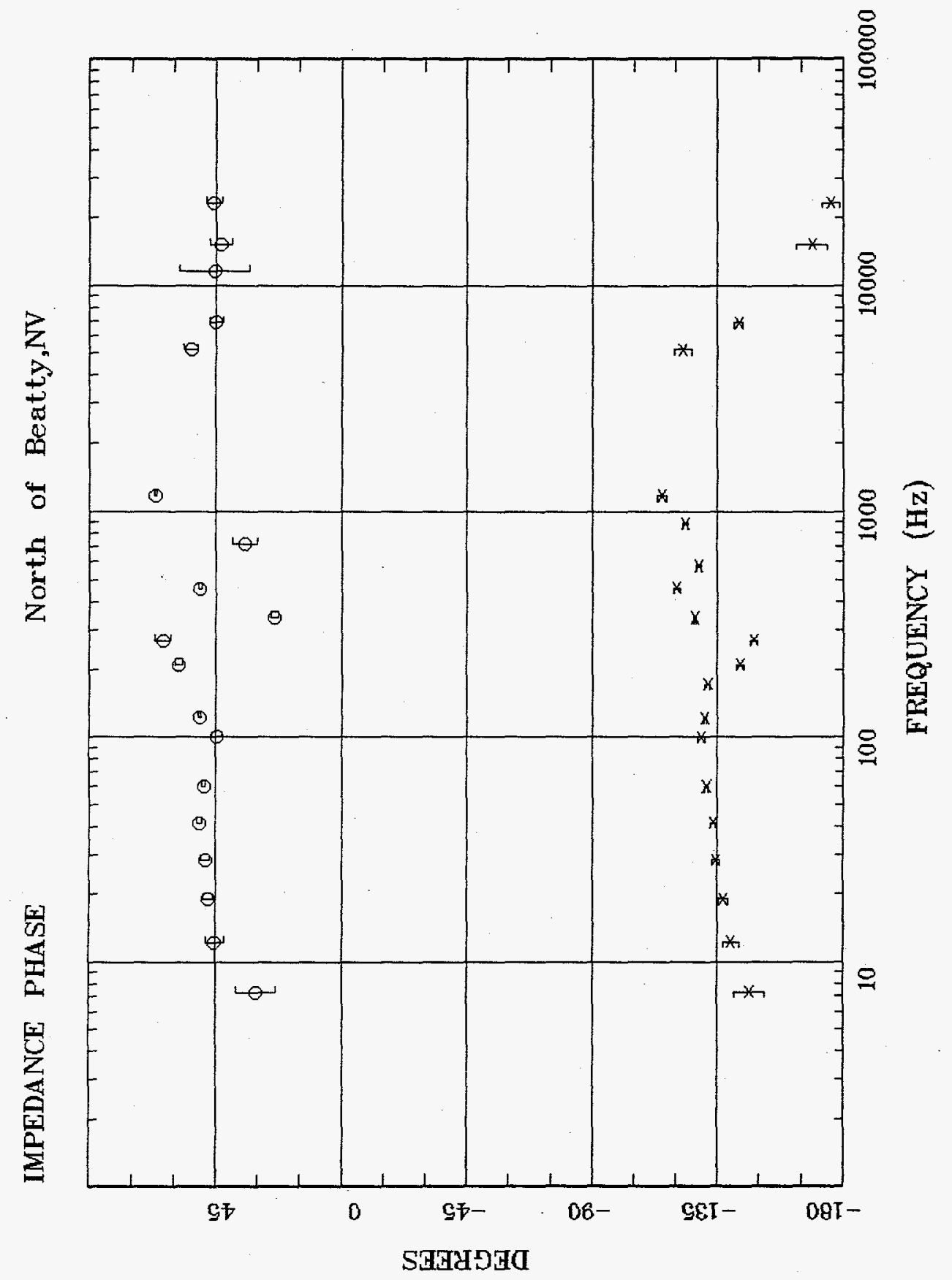

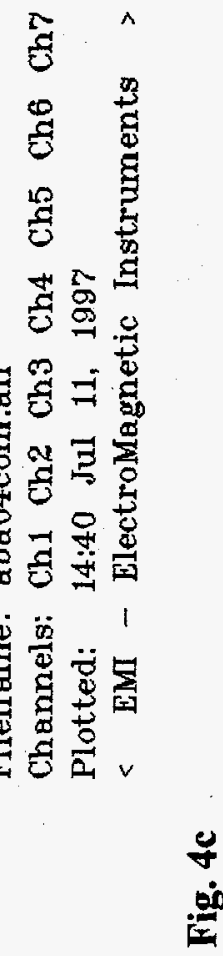

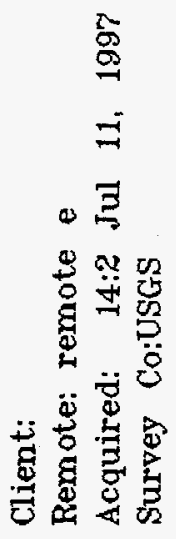




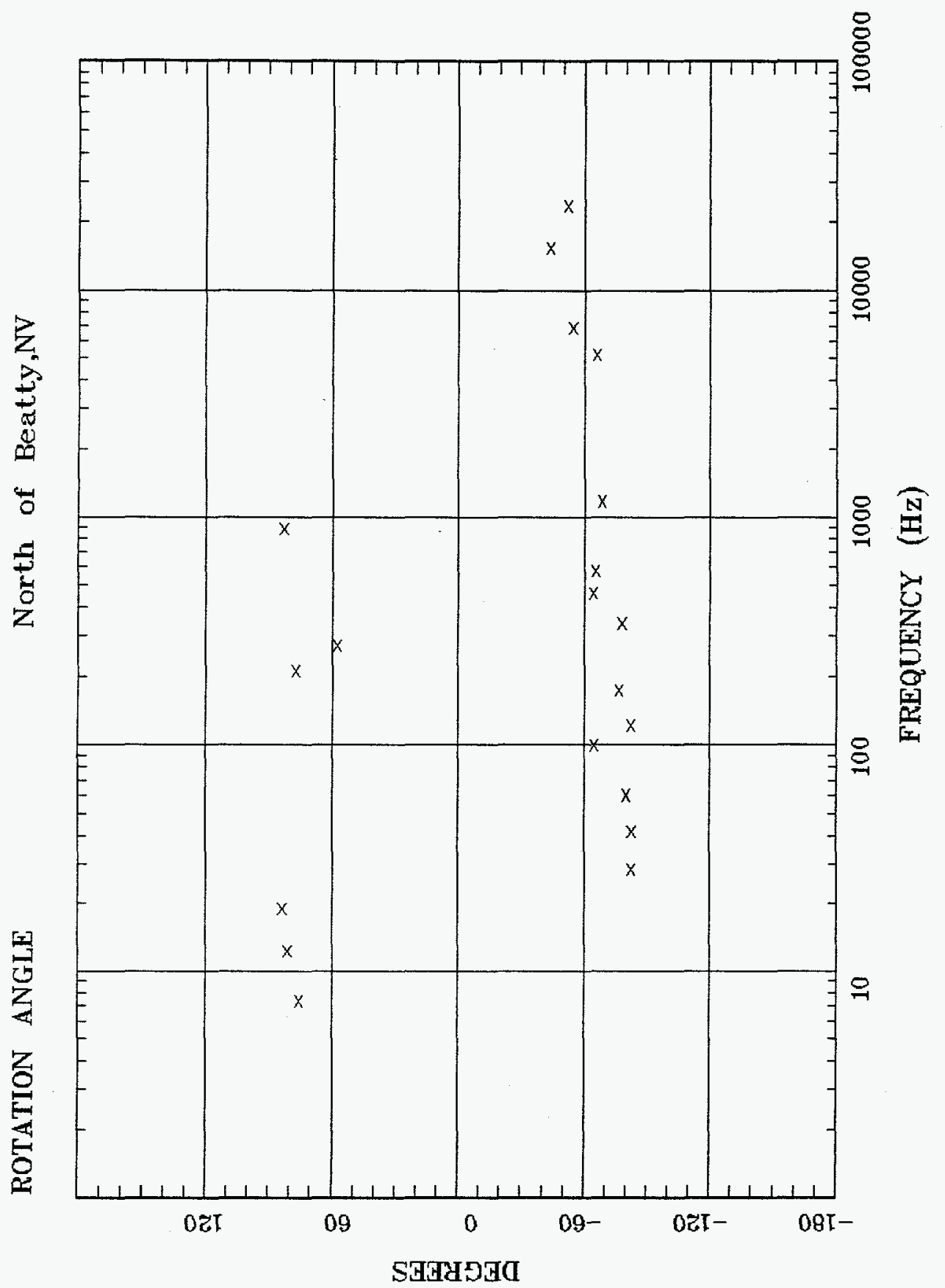

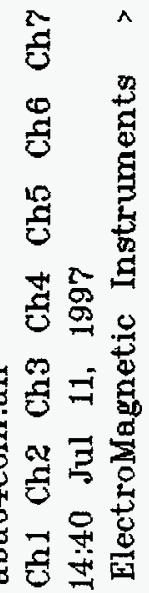

苛离兽

$\underset{8}{8}$

졍

$\exists$

- 3

苍莽

要罂

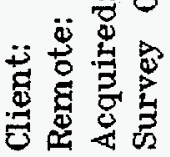


IMPEDANCE SKEW

North of Beatty,NV

హ

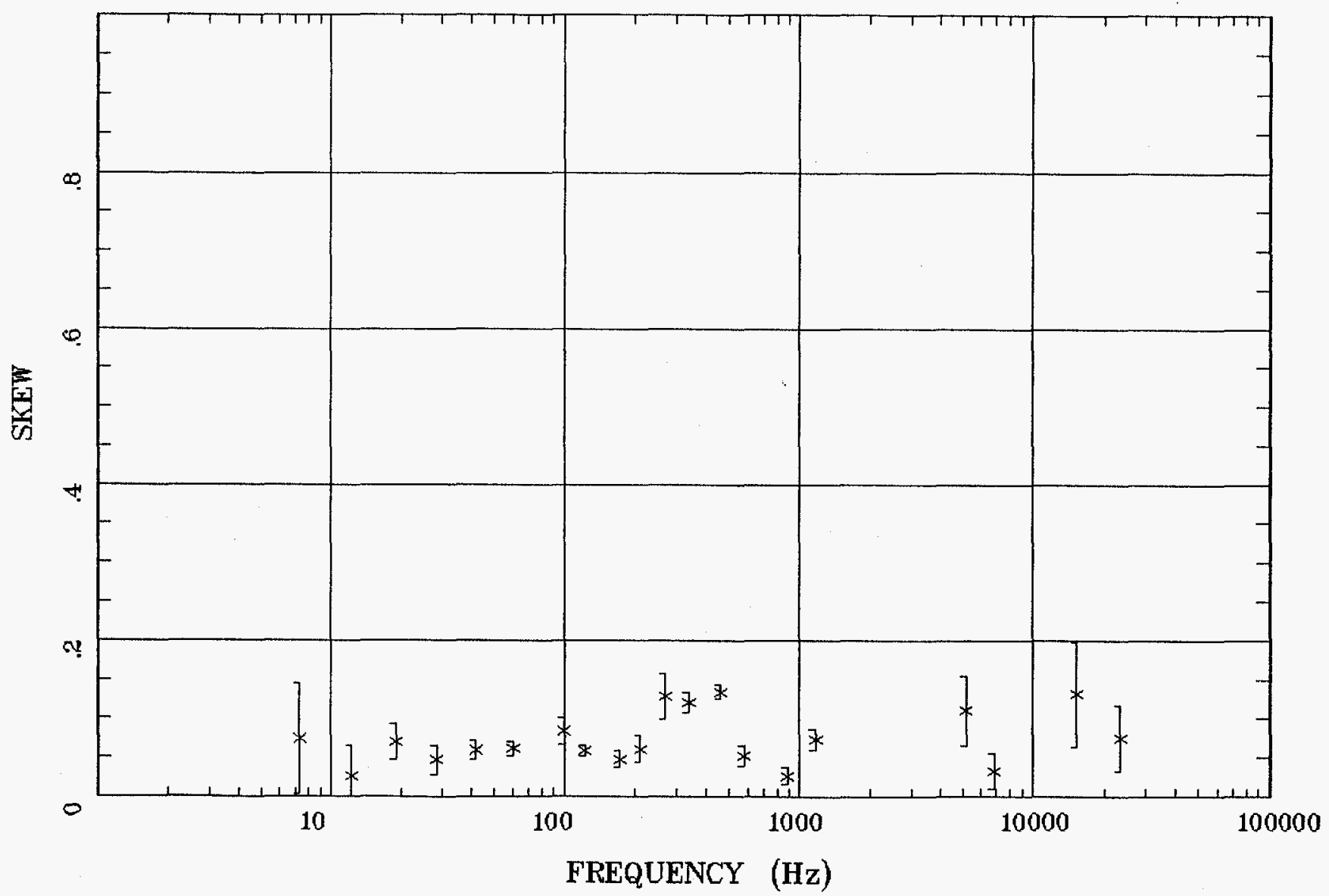

Client:

Remote: remote $\mathrm{e}$

Acquired: 14:2 Jul 11, 1997

Survey Co:USGS

Rotation:

Filename: aba04com.all

Channels: Ch1 Ch2 Ch3 Ch4 Ch5 Ch6 Ch7

Plotted: 14:41 Jul 11, 1997

< EMI - ElectroMagnetic Instruments >

Fig. 4e 


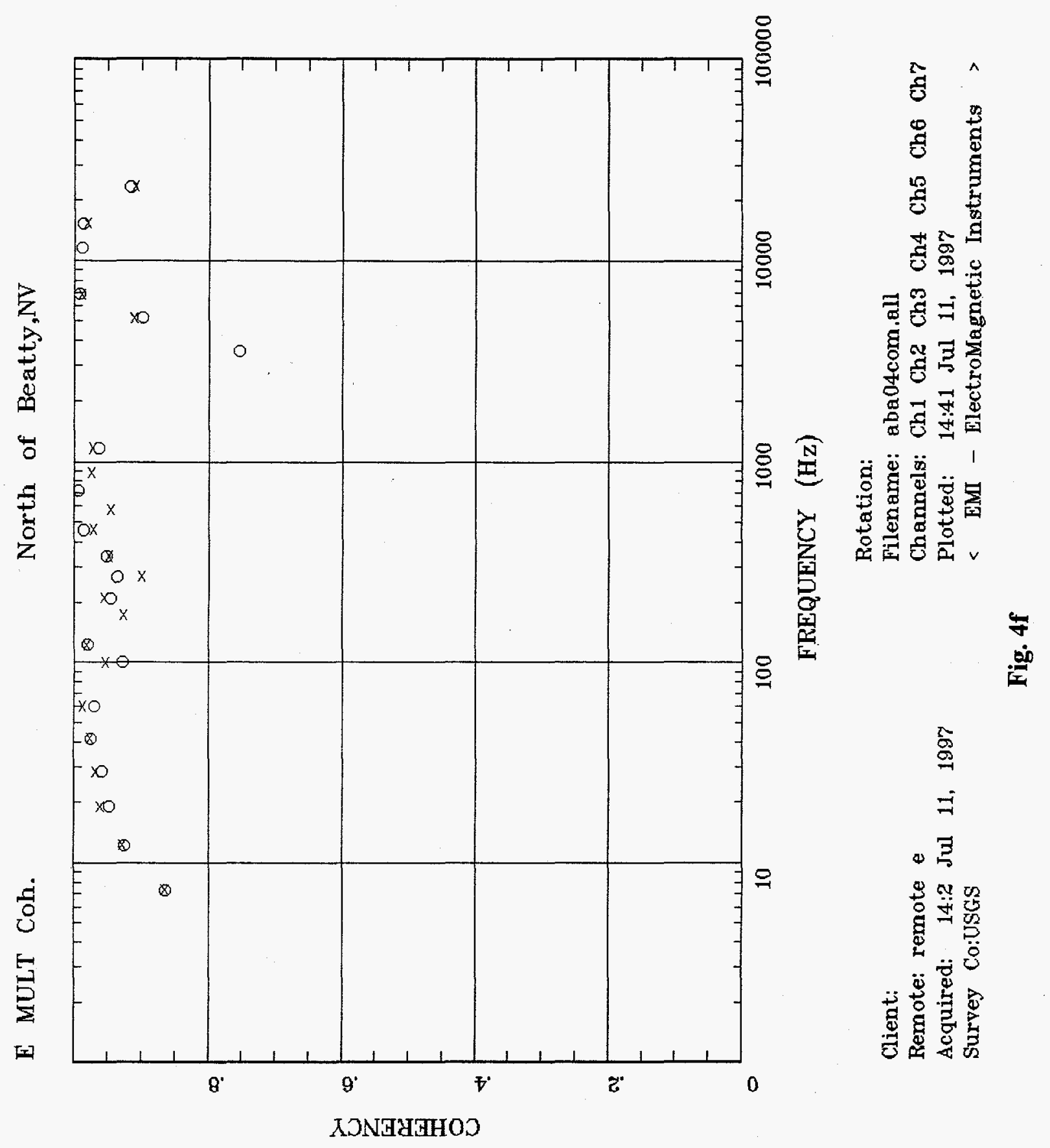




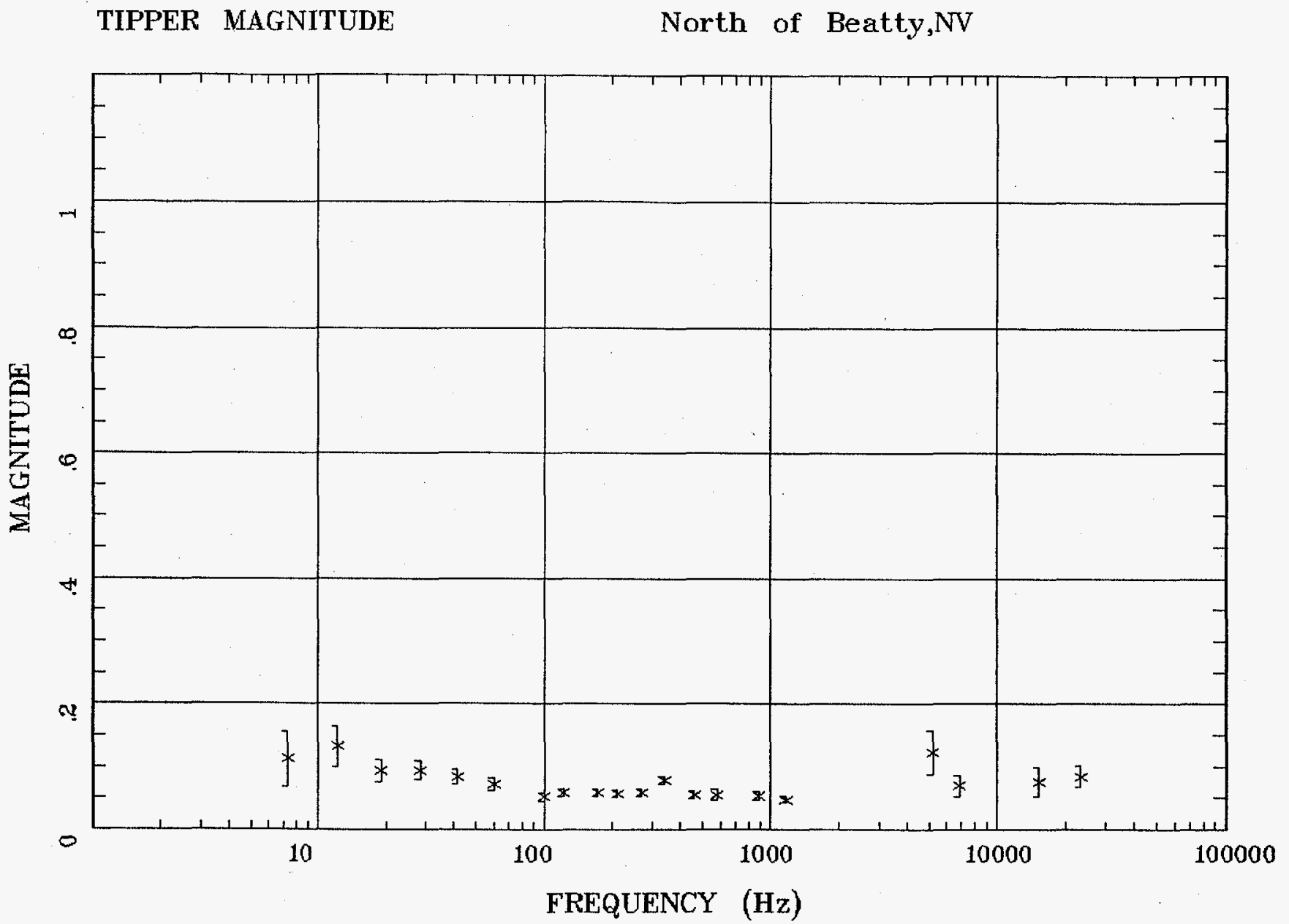

Client:

Remote: remote $\mathrm{e}$

Acquired: 14:2 Jul 11, 1997
Survey Co:USGS

Filename: aba04com.all

Channels: Ch1 Ch2 Ch3 Ch4 Ch5 Ch6 Ch7

Plotted: 14:41 Jul 11, 1997

< EMI - ElectroMagnetic Instruments >

Fig. 4g 


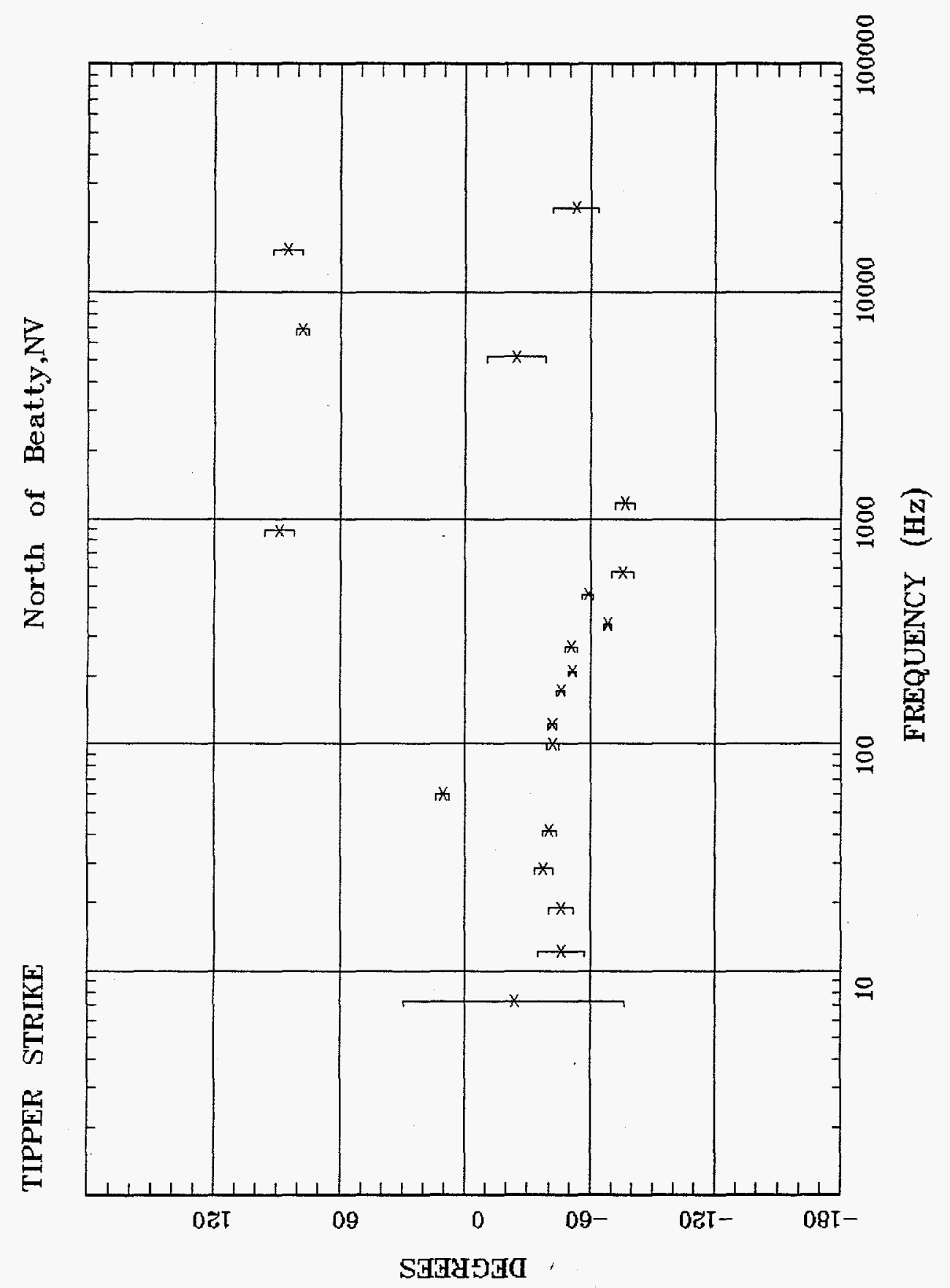

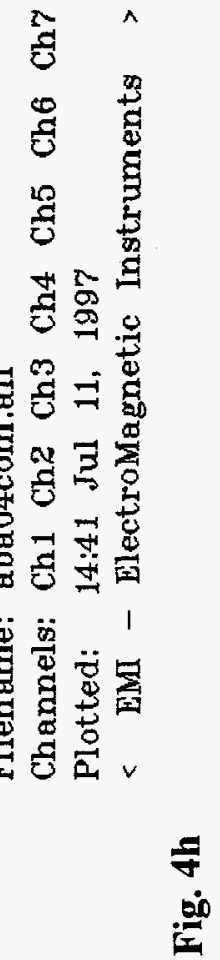

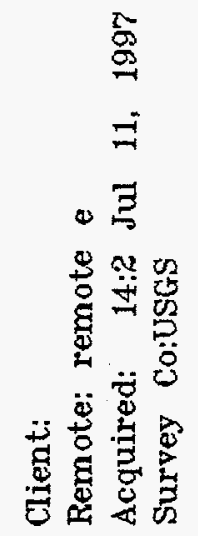


North of Beatty,NV

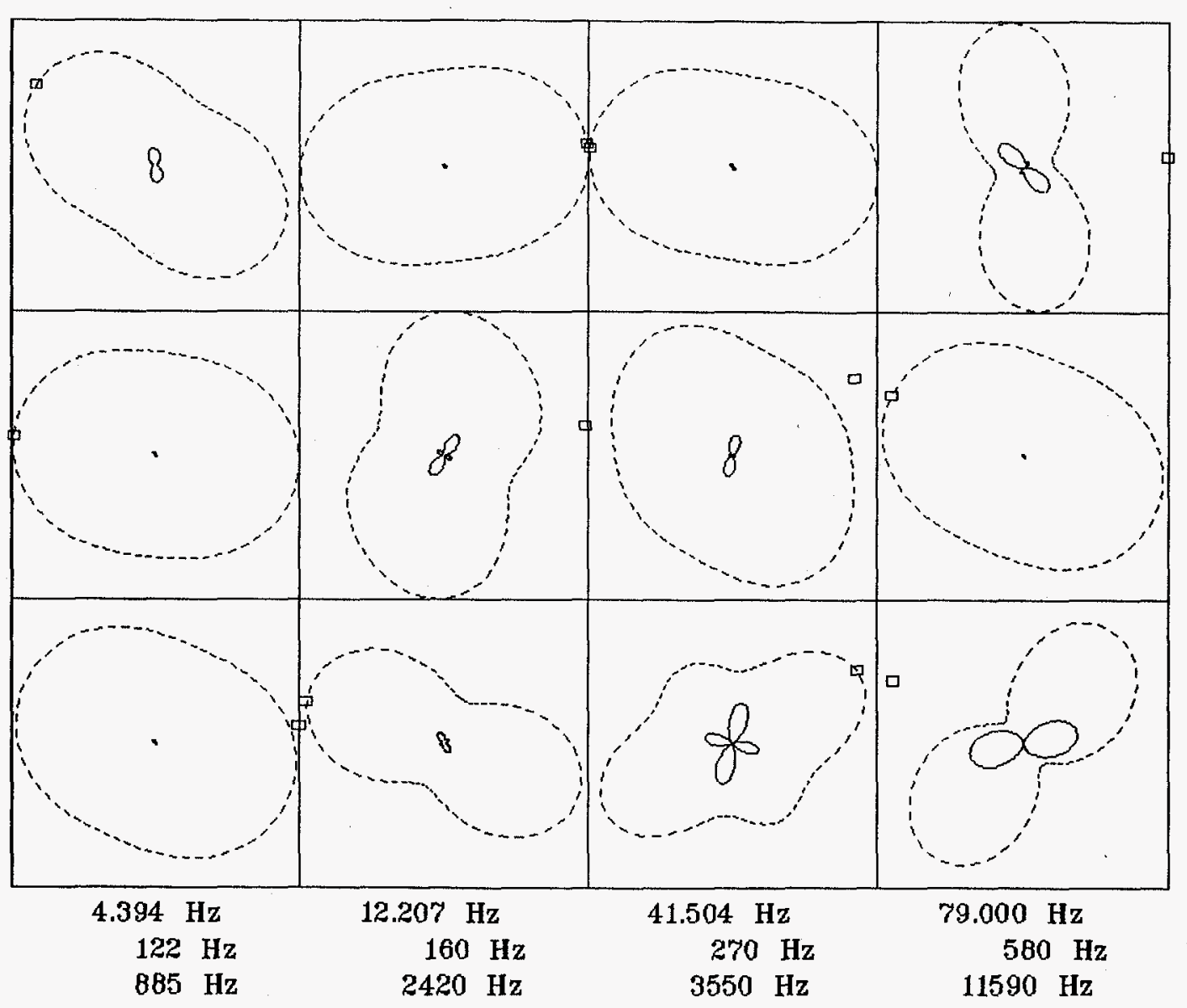

\section{Client:}

Remote: remote e

Acquired: 14:2 Jul 11, 1997 Survey Co:USGS

\section{Rotation:}

Filename: aba04com.all

Channels: Ch1 Ch2 Ch3 Ch4 Ch5 Ch6 Ch7

Plotted: 14:41 Jul 11, 1997

< EMI - ElectroMagnetic Instruments >

Fig. 4i 
Resistivity (Bostick Inv.)

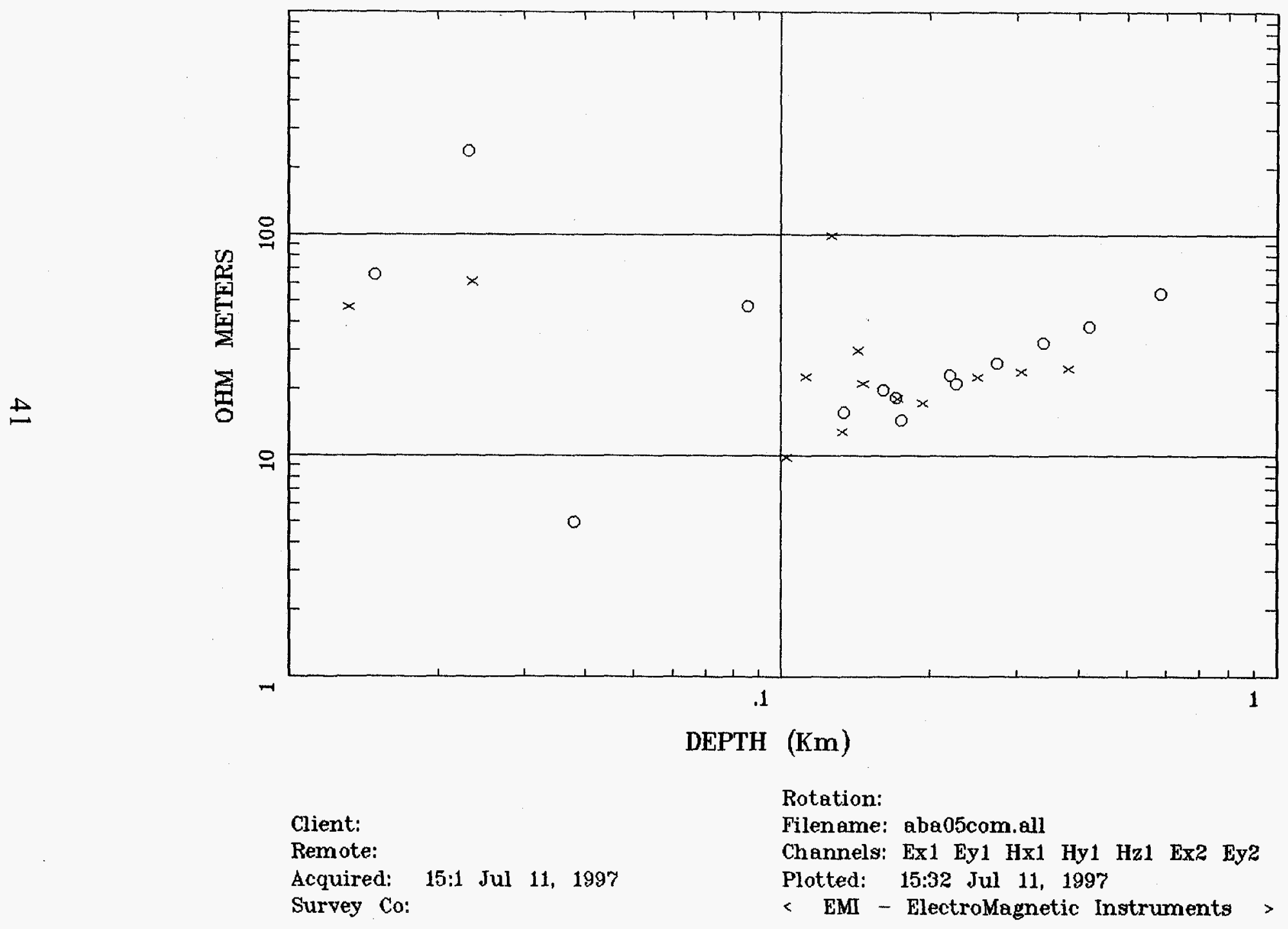

Fig. 5a 
APPARENT RESISTIVITY

north of Beatty

$\vec{N}$

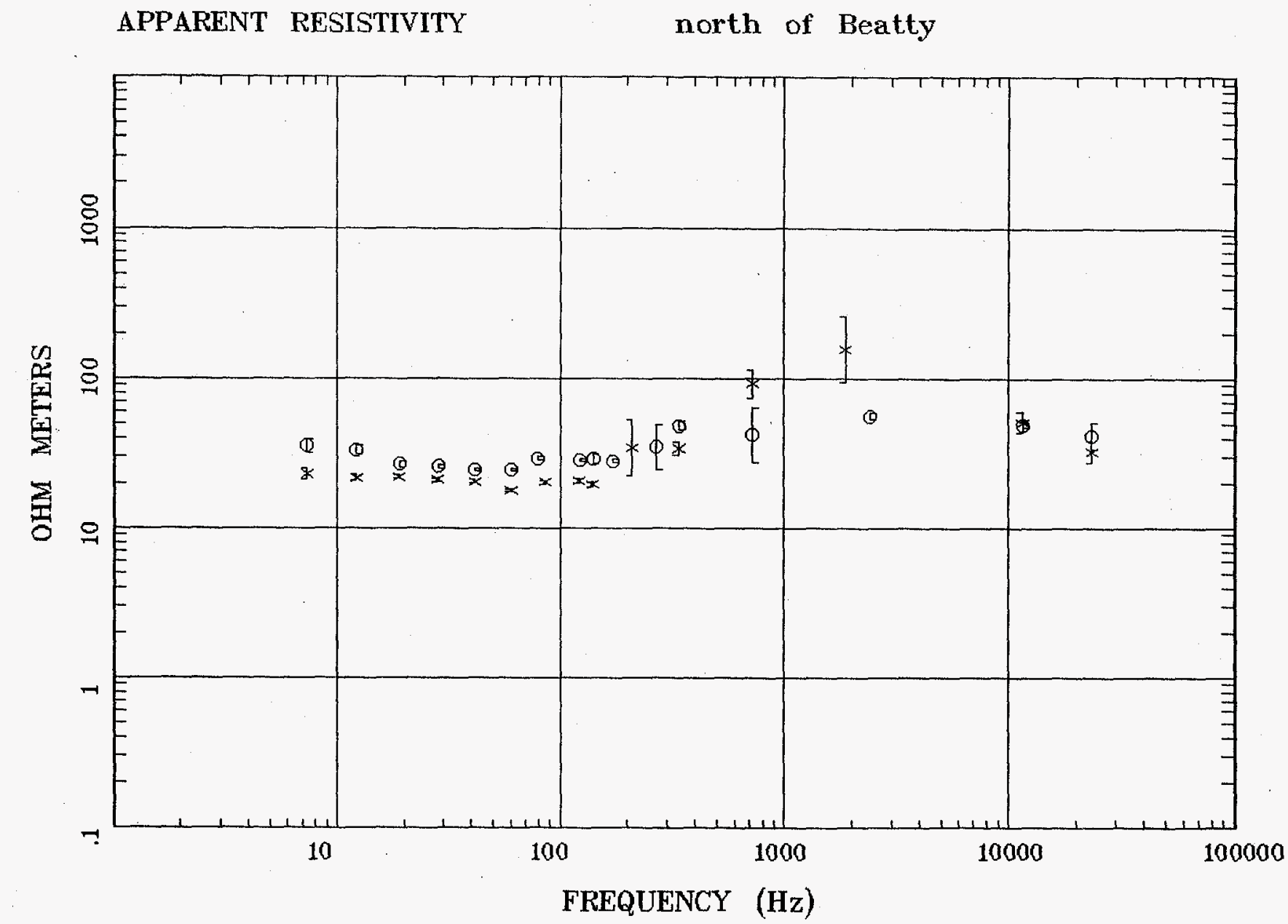

Client:

Remote: remote $\mathrm{e}$

Acquired: 15:1 Jul 11, 1997

Survey Co:USGS

Rotation:

Filename: aba05com.all

Channels: Ch1 Ch2 Ch3 Ch4 Ch5 Ch6 Ch7

Plotted: 15:32 Jul 11, 1997

< EMI - ElectroMagnetic Instruments

Fig. 5b 
north of Beatty

$\stackrel{A}{\omega}$

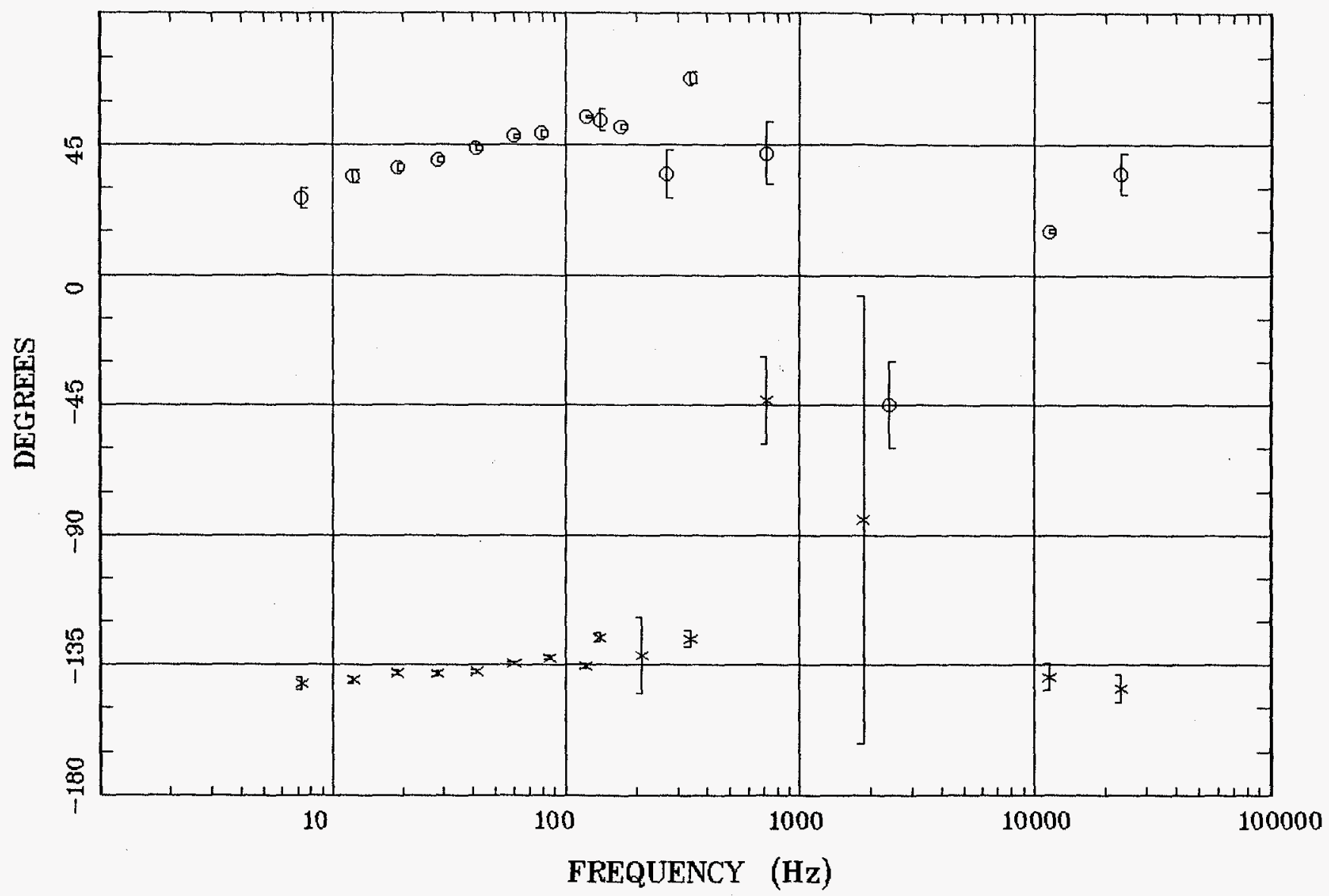

Client:

Remote: remate e

Acquired: 15:1 Jul 11, 1997 Survey Co:USGS
Rotation:

Filename: aba05cam.all

Channels: Ch1 Ch2 Ch3 Ch4 Ch5 Ch6 Ch7 Plotted: 15:32 Jul 11, 1997

< EMI - ElectroMagnetic Instruments >

Fig. 5c 
ROTATION ANGLE

north of Beatty

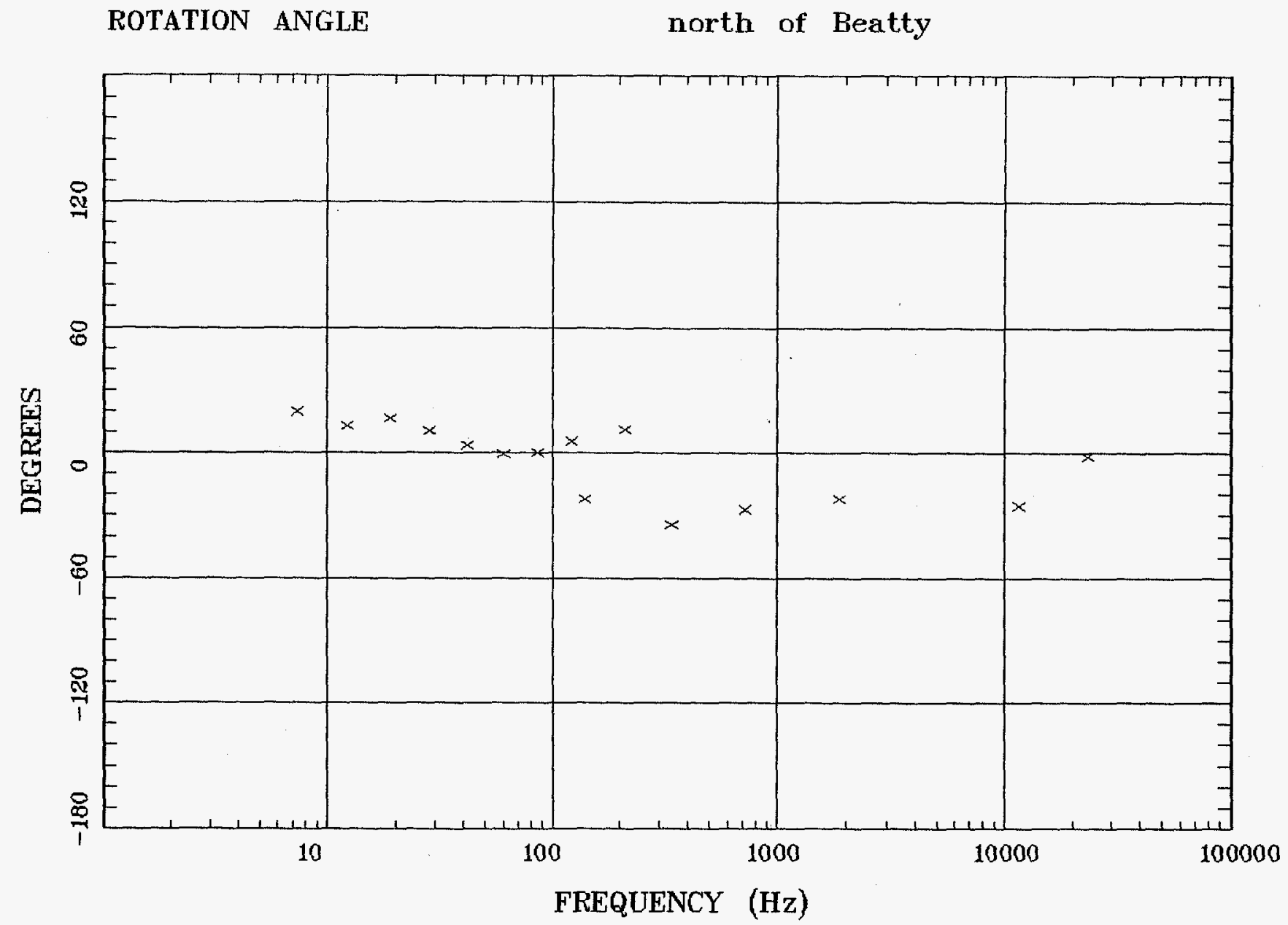

Client:

Rotation:

Remote: remote e

Filename: aba05com.all

Acquired: 15:1 Jul 11, 1997

Channels: Ch1 Ch2 Ch3 Ch4 Ch5 Ch6 Ch7

Survey Co:USGS

Plotted: 15:32 Jul 11, 1997

< EMI - ElectroMagnetic Instruments >

Fig. 5d 


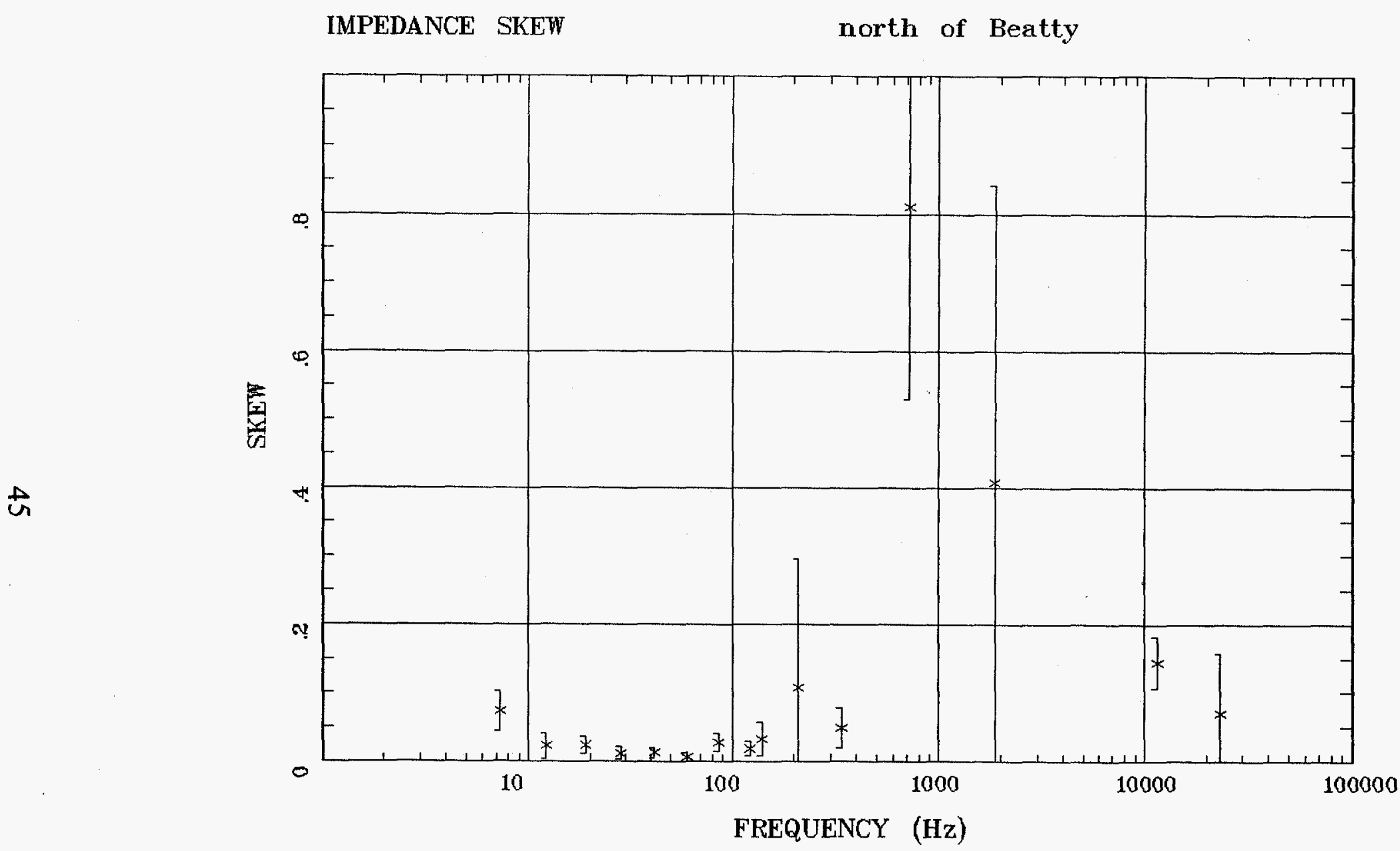

Client:

Remote: remote $e$

Acquired: 15:1 Jul 11, 1997

Survey Co:USGS

Rotation:

Filename: aba05com.all

Channels: Ch1 Ch2 Ch3 Ch4 Ch5 Ch6 Ch7

Plotted: 15:32 Jul 11, 1997

< EMI - ElectroMagnetic Instruments >

Fig. 5e 


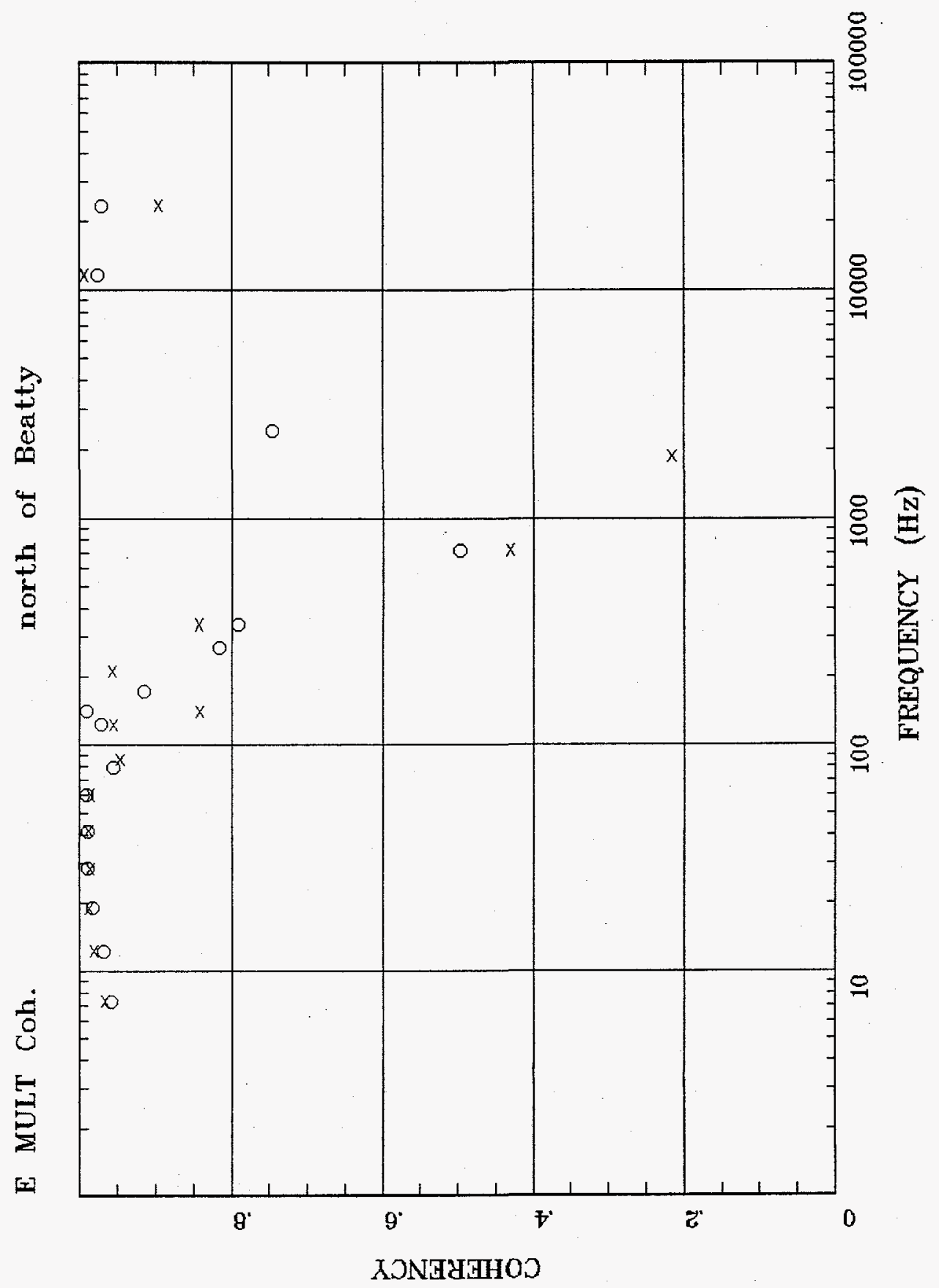

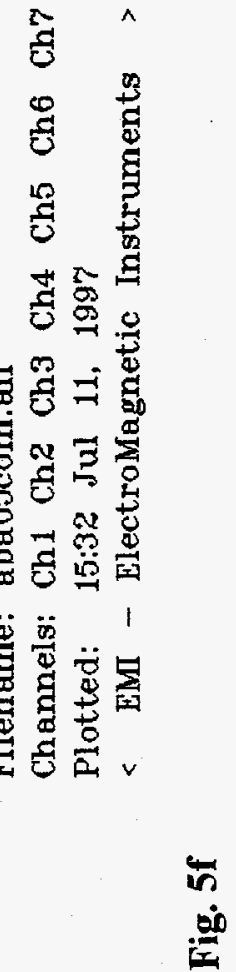

종

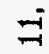

- 3

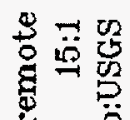

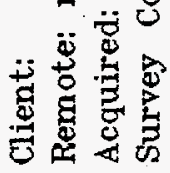
i 


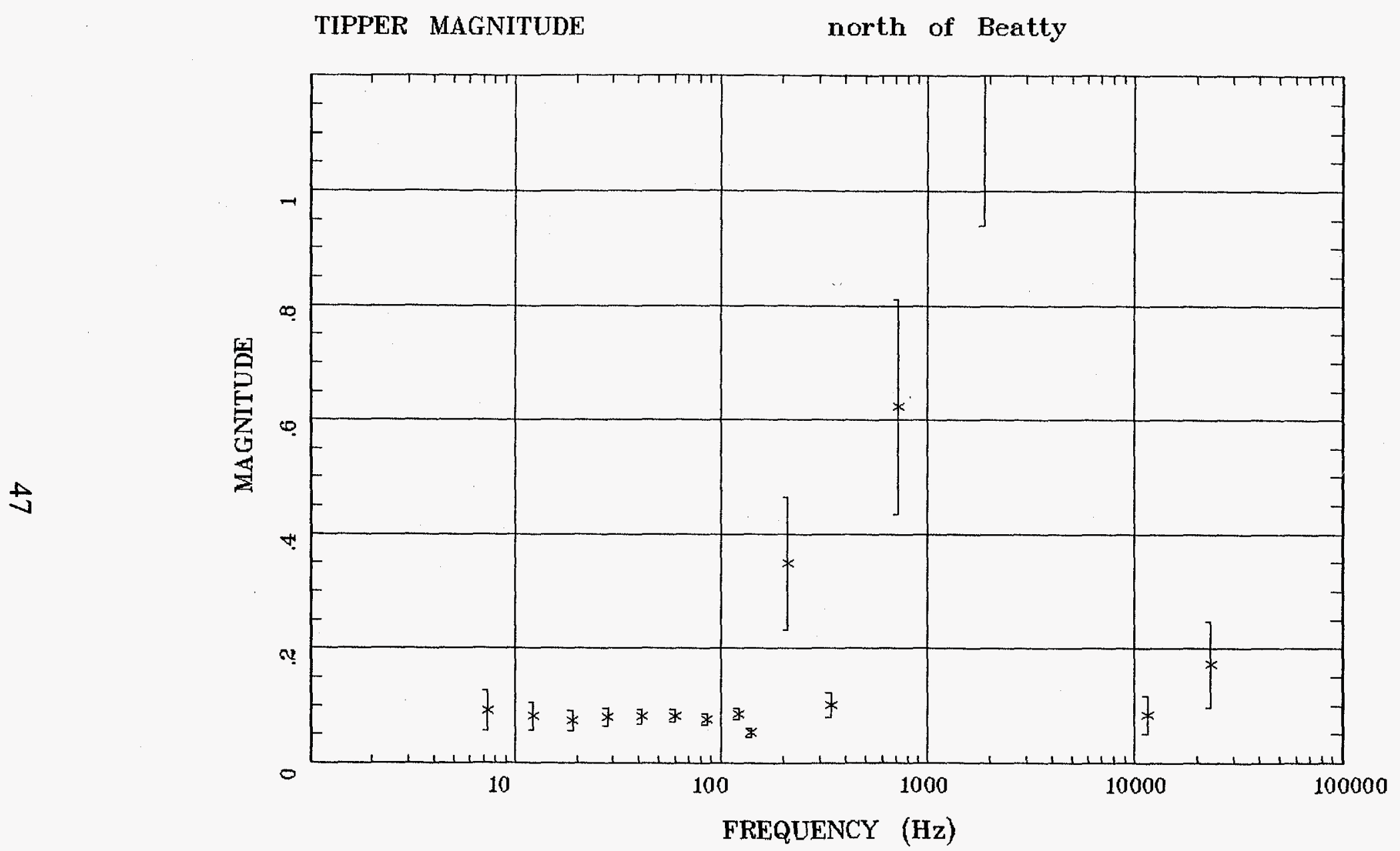

Client:

Remote: remote $\mathrm{e}$

Acquired: 15:1 Jul 11, 1997 Survey Co:USGS
Rotation:

Filename: aba05com.all

Channels: Ch1 Ch2 Ch3 Ch4 Ch5 Ch6 Ch7

Plotted: 15:32 Jul 11, 1997

< EMI - ElectroMagnetic Instruments >

Fig. 5g 
north of Beatty

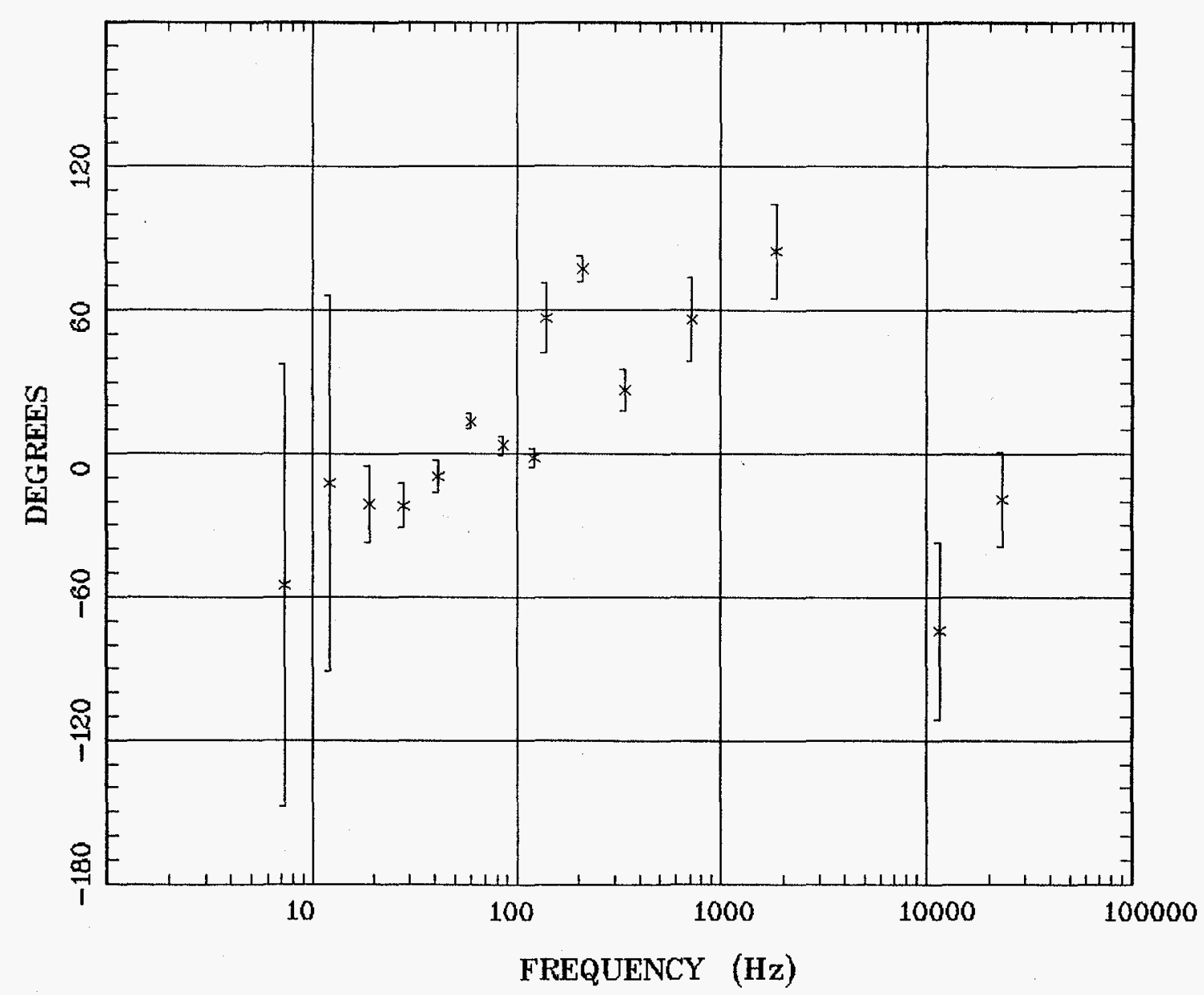

Client:

Remote: remote $\mathrm{e}$ Acquired: 15:1 Jul 11, 1997 Survey Co:USGS
Rotation:

Filename: aba05com.all

Channels: Ch1 Ch2 Ch3 Ch4 Ch5 Ch6 C:

Plotted: 15:32 Jul 11, 1997

< EMI - ElectroMagnetic Instruments

Fig. $5 \mathrm{~h}$ 


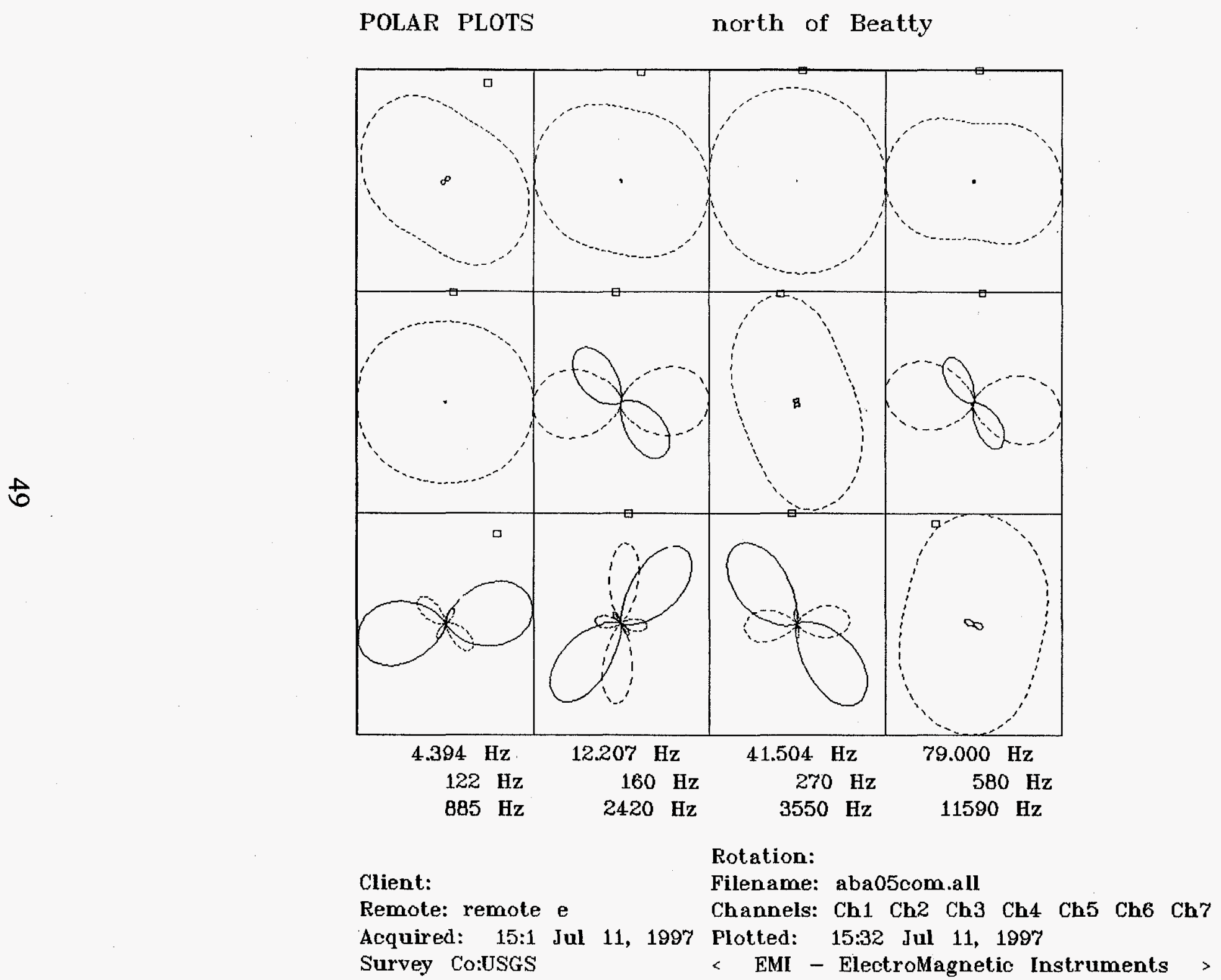

Fig. 5i 


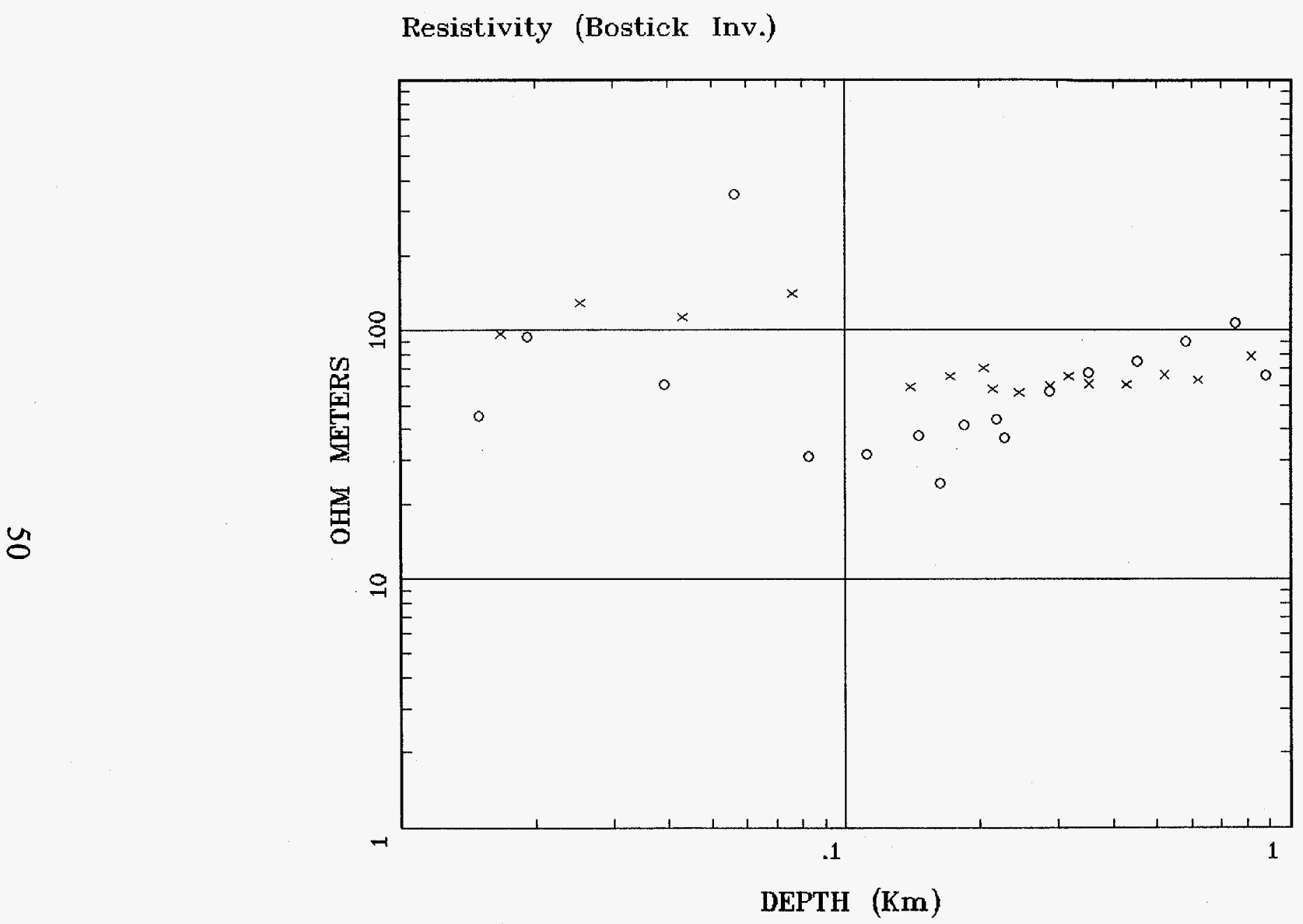

Client:

Remote:

Acquired: $21: 3$ Jun 10, 1997

Survey Co:
Rotation:

Filename: aba06com.all

Channels: Ex1 Ey1 Hx1 Hy1 Hz1 Ex2

Plotted: 14:11 Jul 10, 1997

< EMI - ElectroMagnetic Instruments

Fig. 6a 


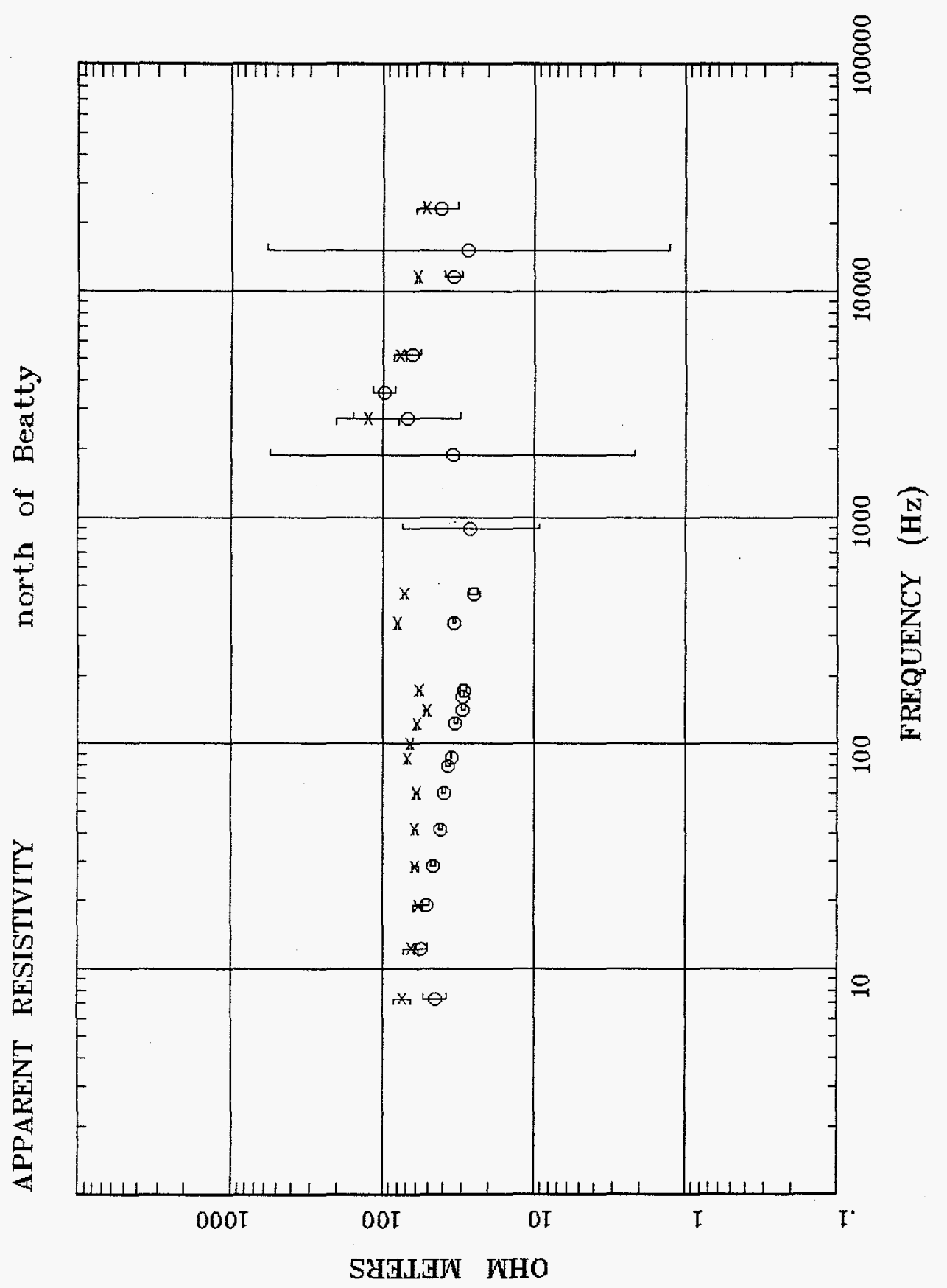

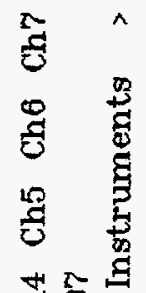

过

ซ류

g.

8 㝴寻

总忌焉

$\ddot{0} \ddot{0} \quad \mid$

要哥焉

원 홍

足要

空
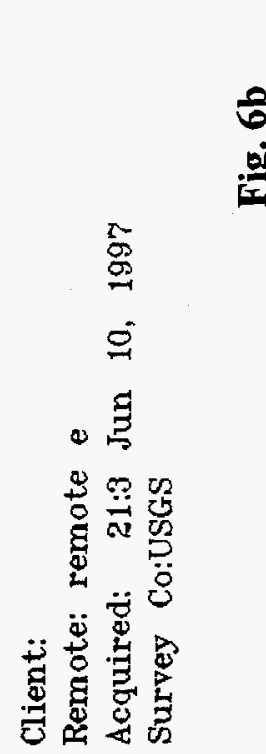

bo

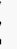

요

$\stackrel{9}{3}$

导骂

幽

$\ddot{0}$

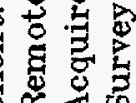

NHO 
zS

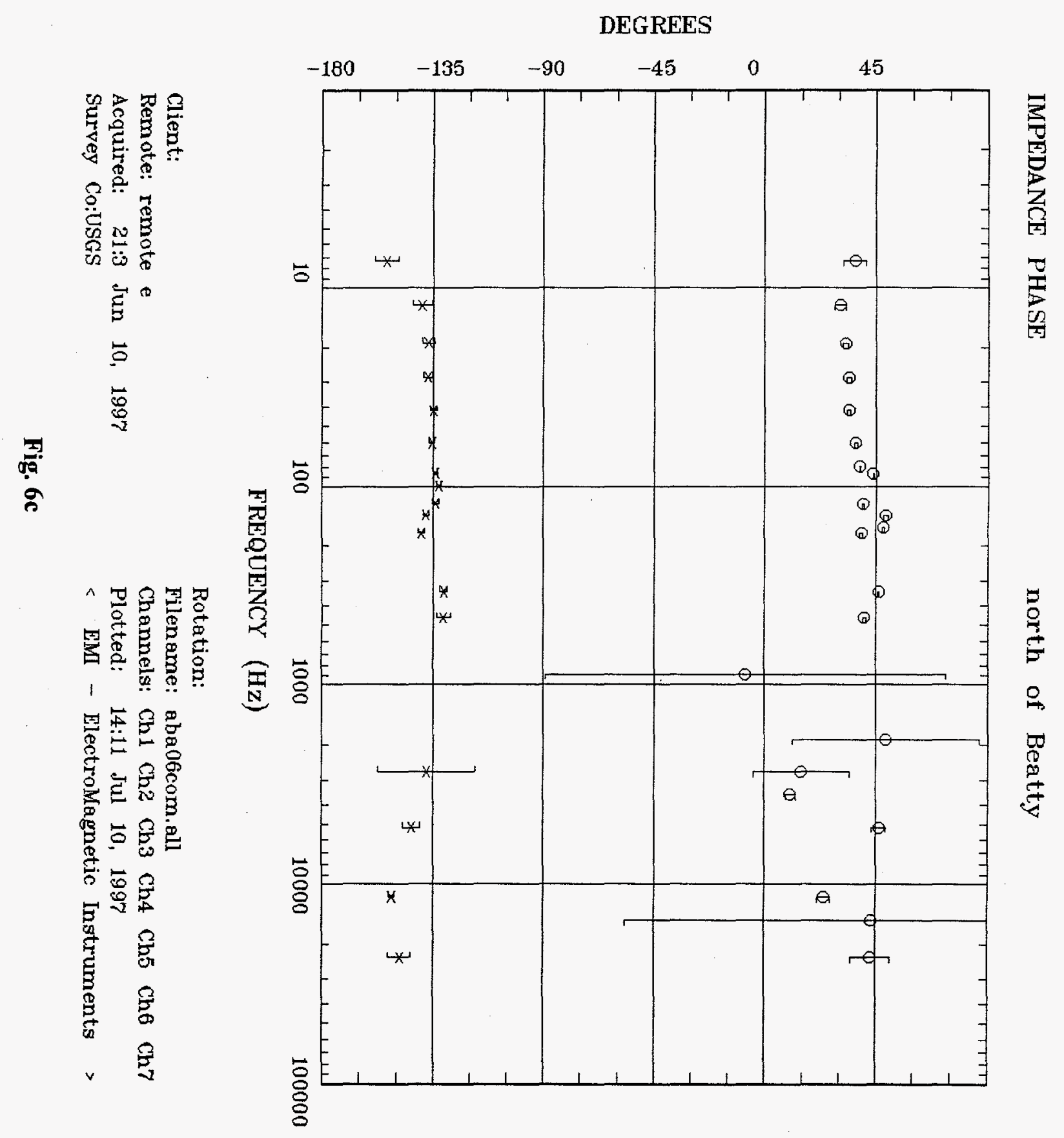



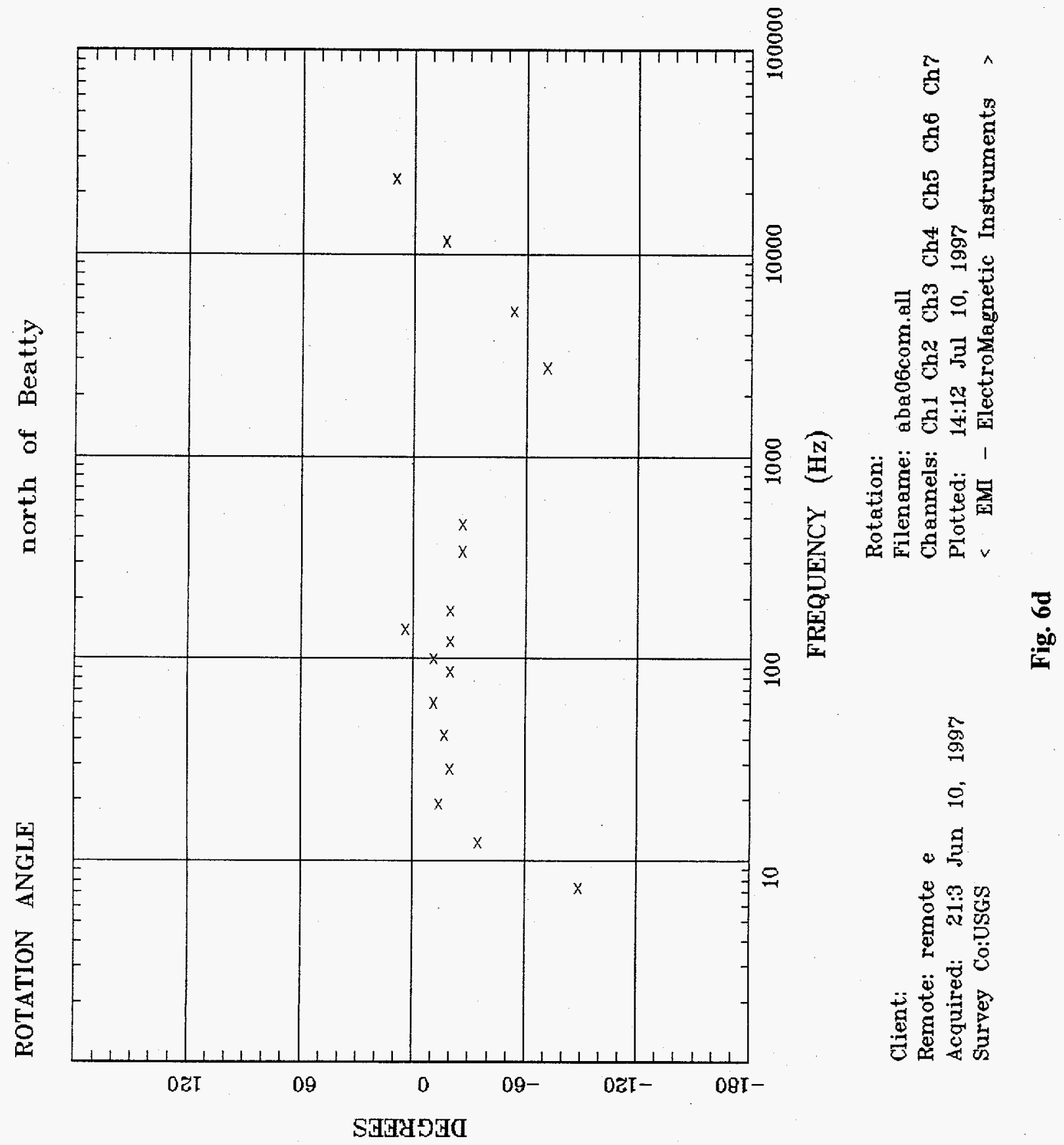
IMPEDANCE SKEW

north of Beatty

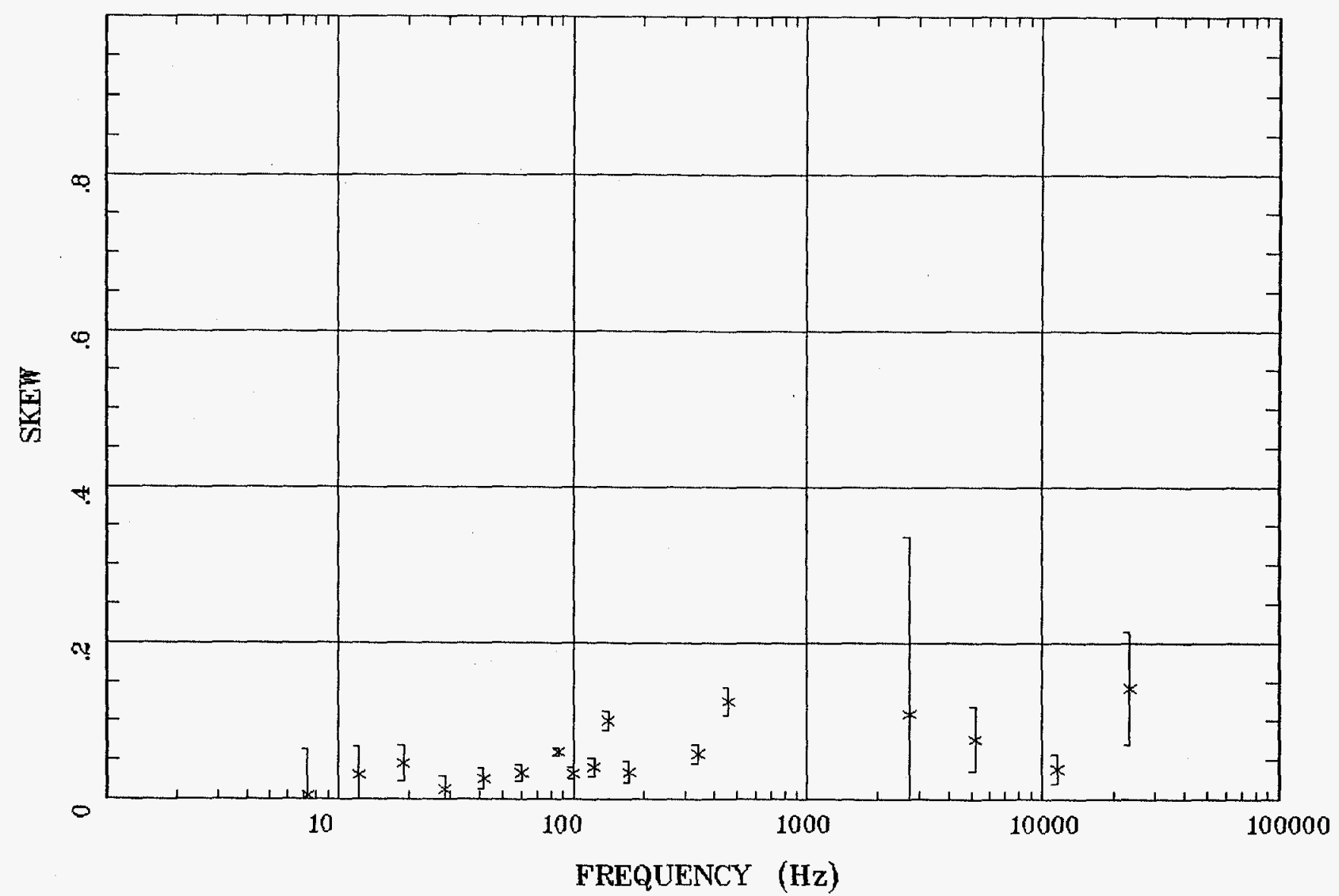

Client:

Rotation

Remote: remote e

Acquired: 21:3 Jun 10, 1997

Survey Co:USGS

Filename: aba06cam.all

Channels: Ch1 Ch2 Ch3 Ch4 Ch5 Ch6 Ch7

Platted: 14:12 Jul 10, 1997

< EMI - ElectroMagnetic Instruments >

Fig. 6e 


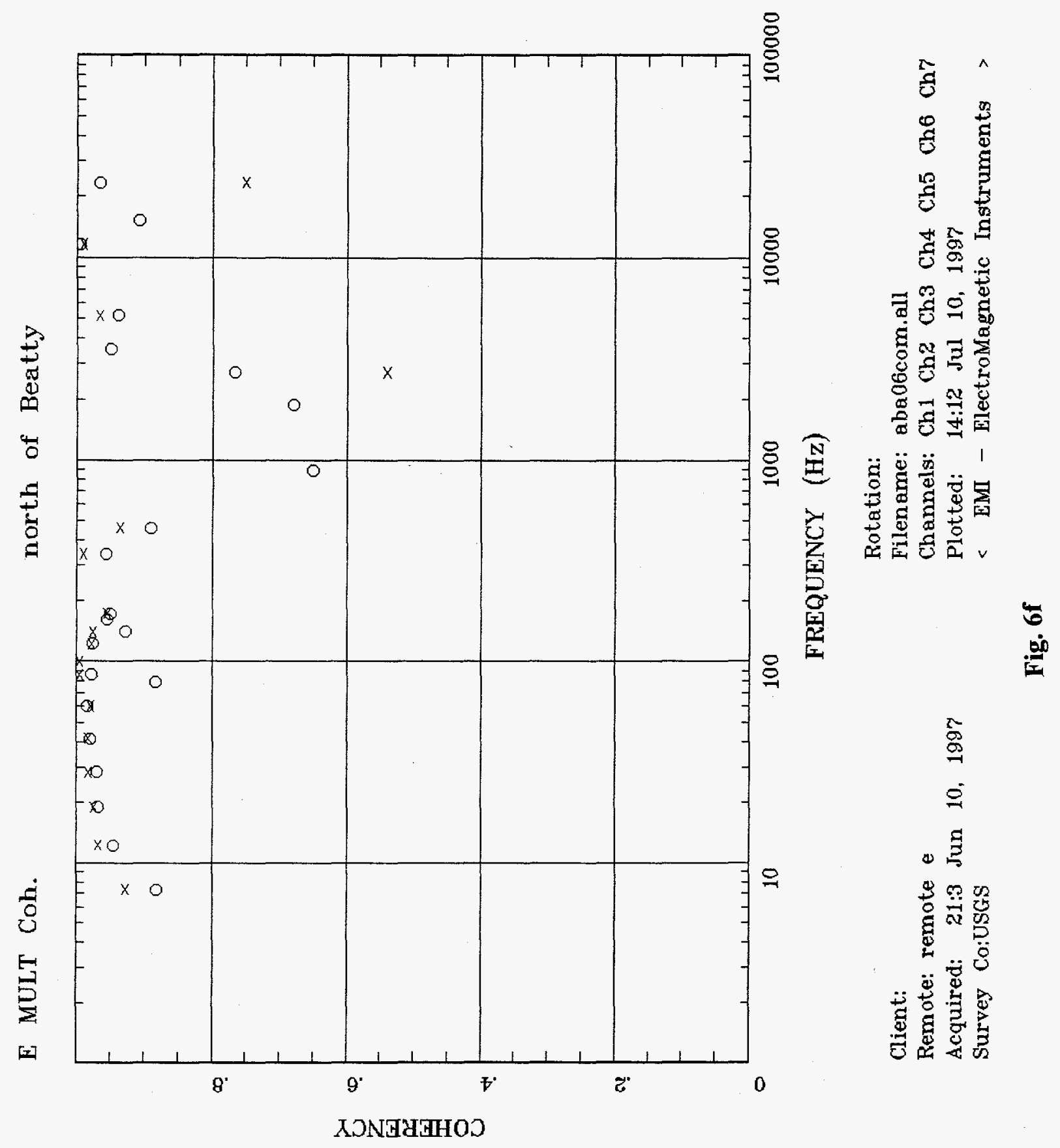




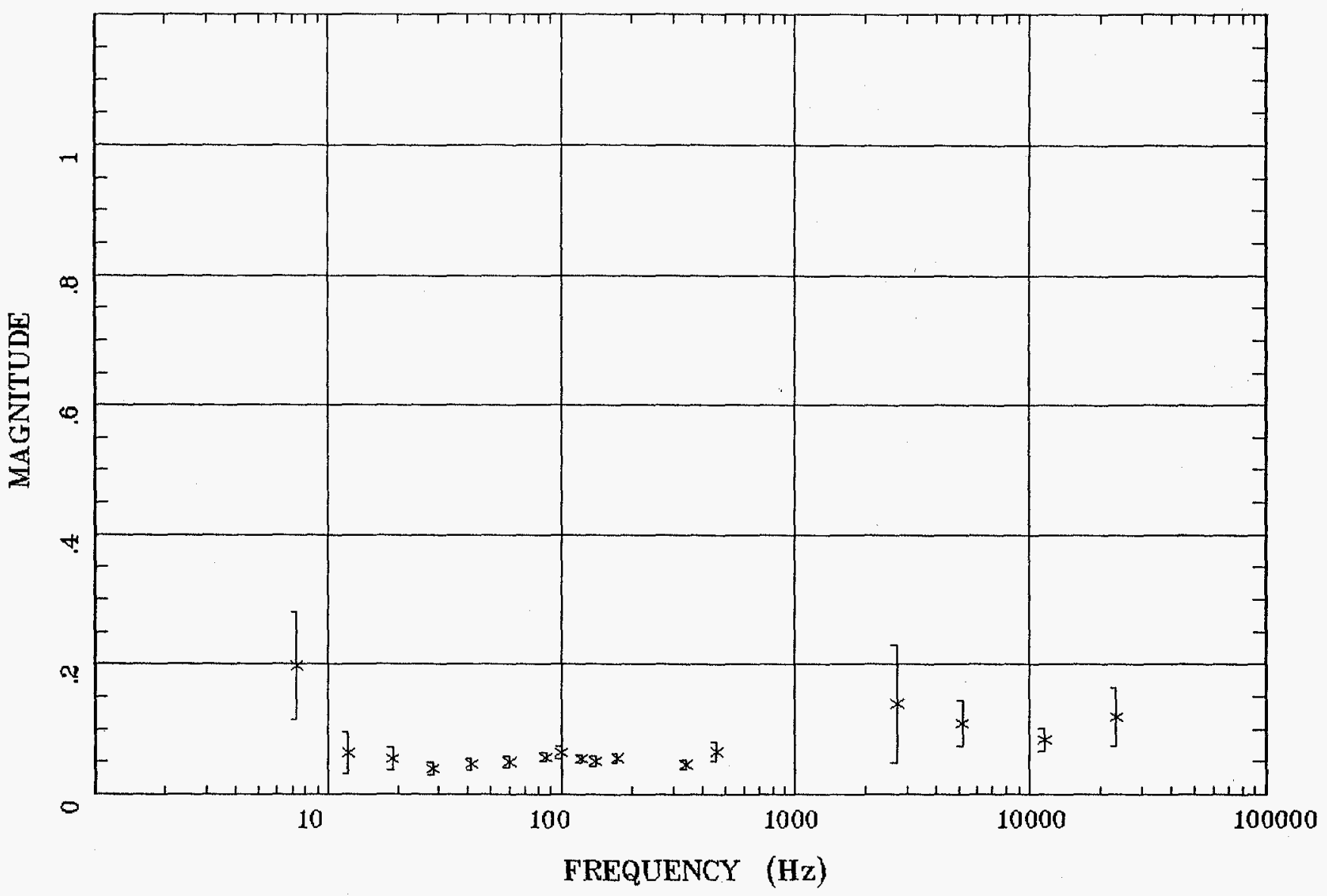

Client:

Rotation:

Remote: remote e

Filename: aba06com.all

Acquired: 21:3 Jun 10, 1997

Channels: Ch1 Ch2 Ch3 Ch4 Ch5 Ch6 Ch7

Plotted: 14:12 Jul 10, 1997

Survey Co:USGS

< EMI - ElectroMagnetic Instruments >

Fig. 6g 
north of Beatty

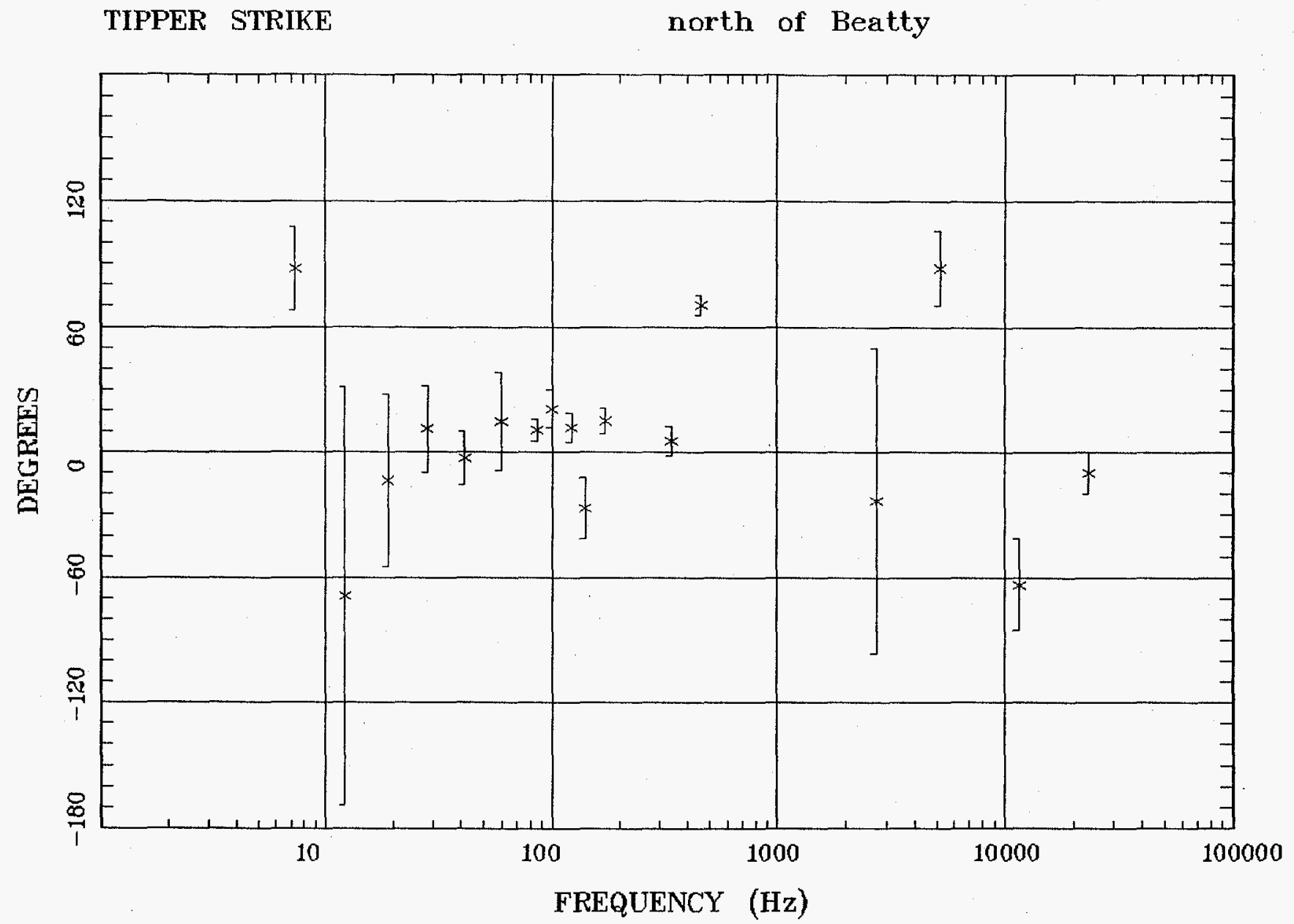

Client:

Remote: remote

Acquired: 21:3 Jun 10, 1997

Survey Co:USGS
Rotation:

Filename: aba06com.all

Channels: Ch1 Ch2 Ch3 Ch4 Ch5 Ch6 Ch7

Plotted: 14:12 Jul 10, 1997

< EMI - ElectroMagnetic Instruments >

Fig. 6h 


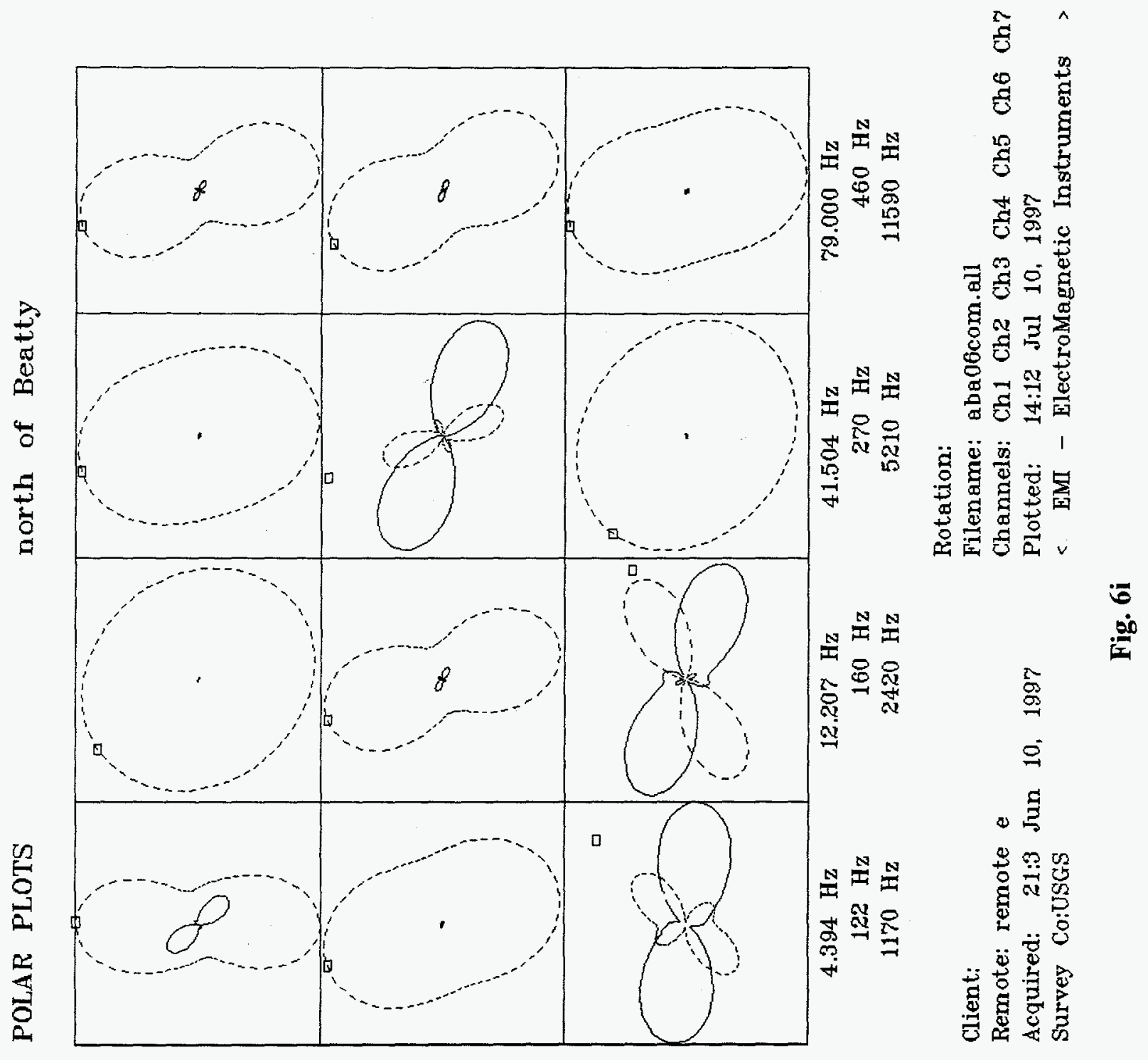


Resistivity (Bostick Inv.)

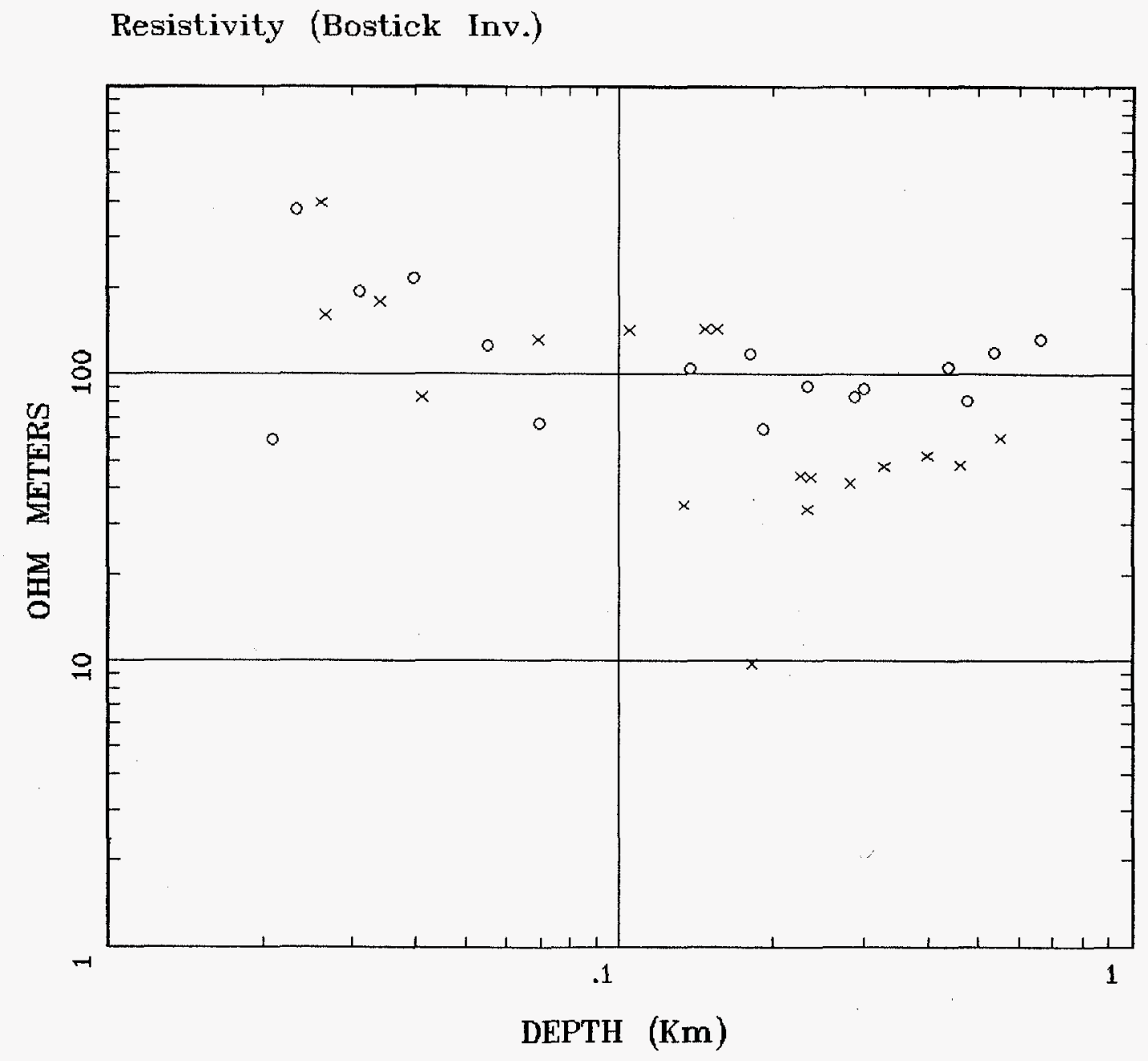

Client:

Remote:

Acquired: 21:2 Jun 09, 1997 Survey Co:
Rotation:

Filename: aba07comb.all

Channels: Ex1 Ey1 Hx1 Hy1 Hz1 Ex2

Plotted: 20:02 Jun 10, 1997

< EMI - ElectroMagnetic Instruments

Fig. 7a 


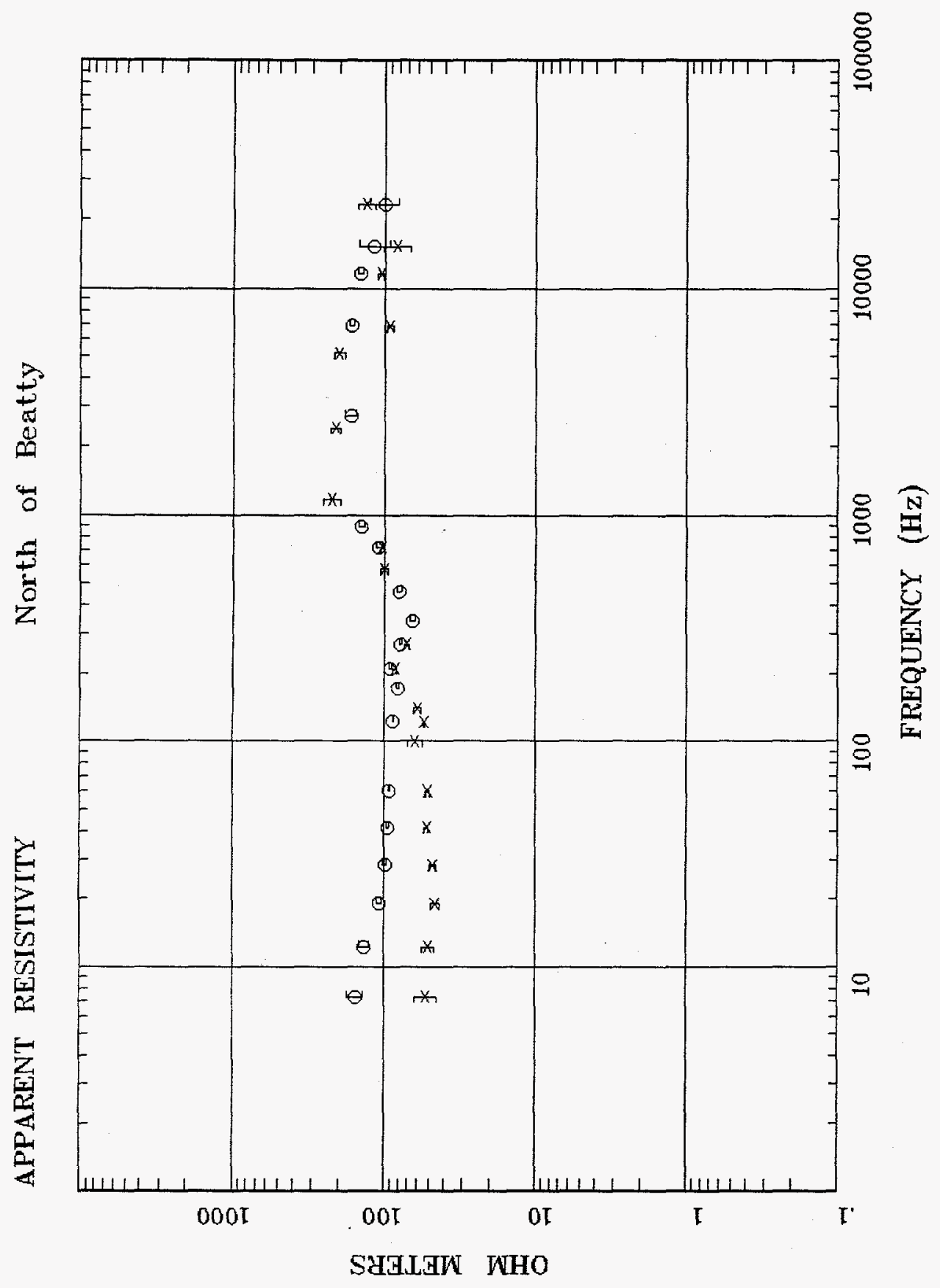

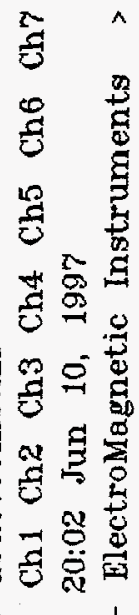

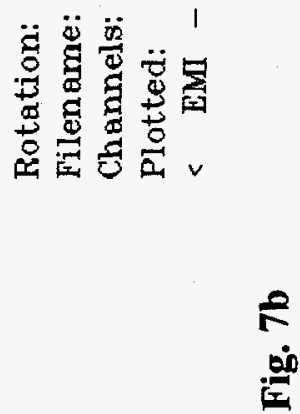

옹

8

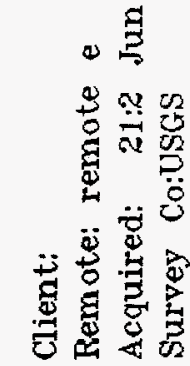

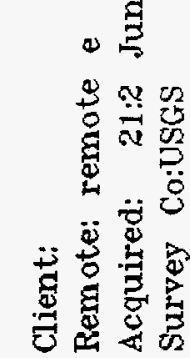

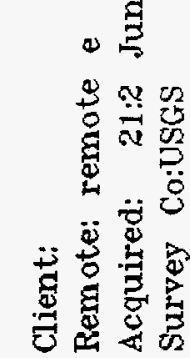

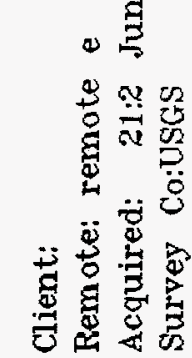

-

SAGIGW NHO 


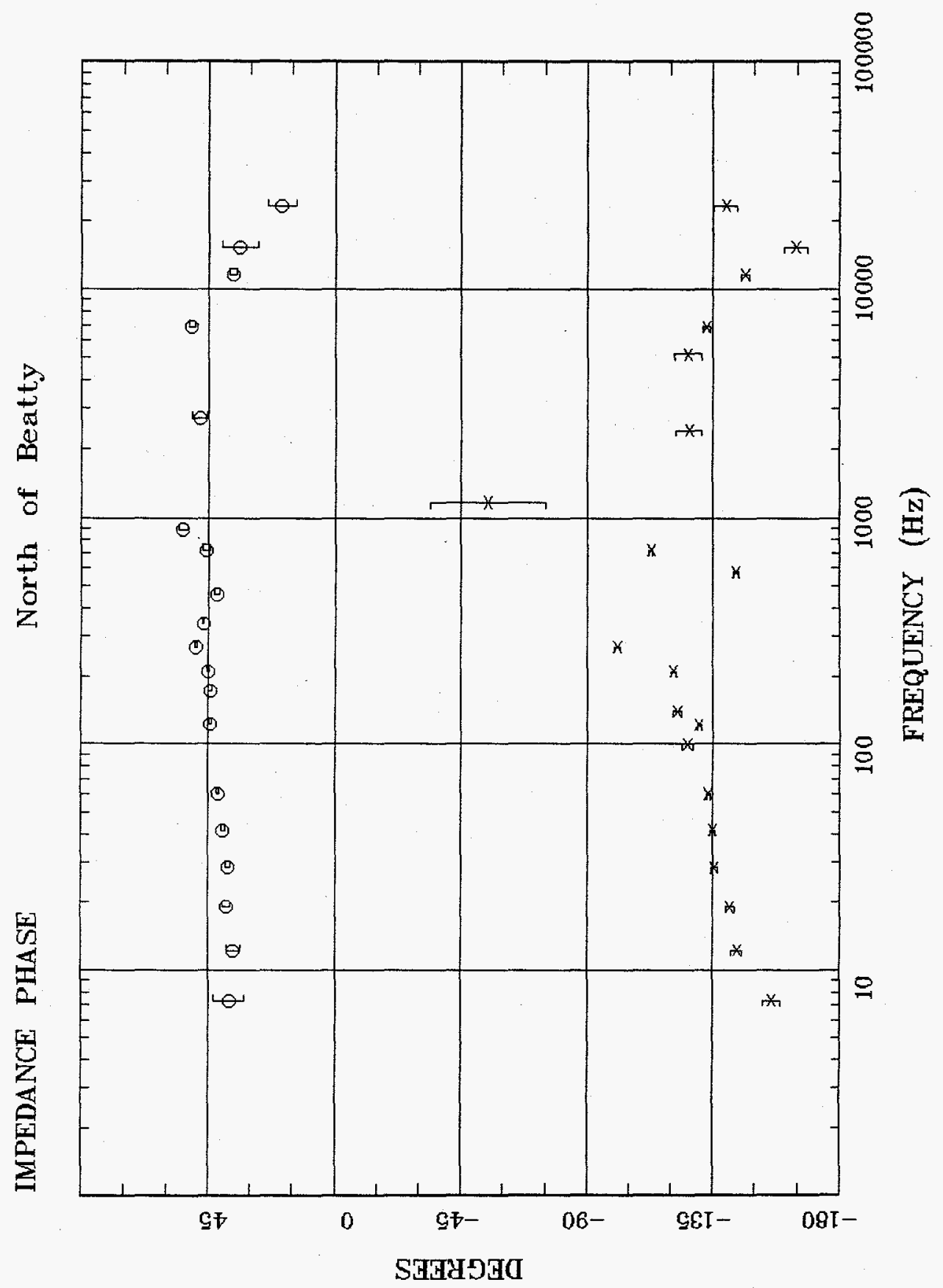

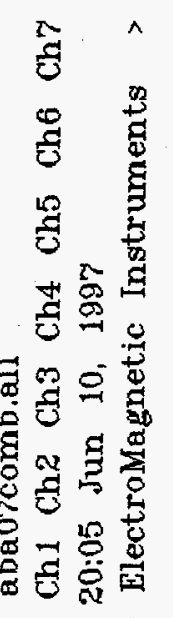

范苟

票票焉

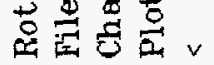

i

in

꽁

8

-

总哭皆

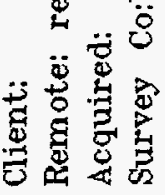

ऽАЯसगНО 


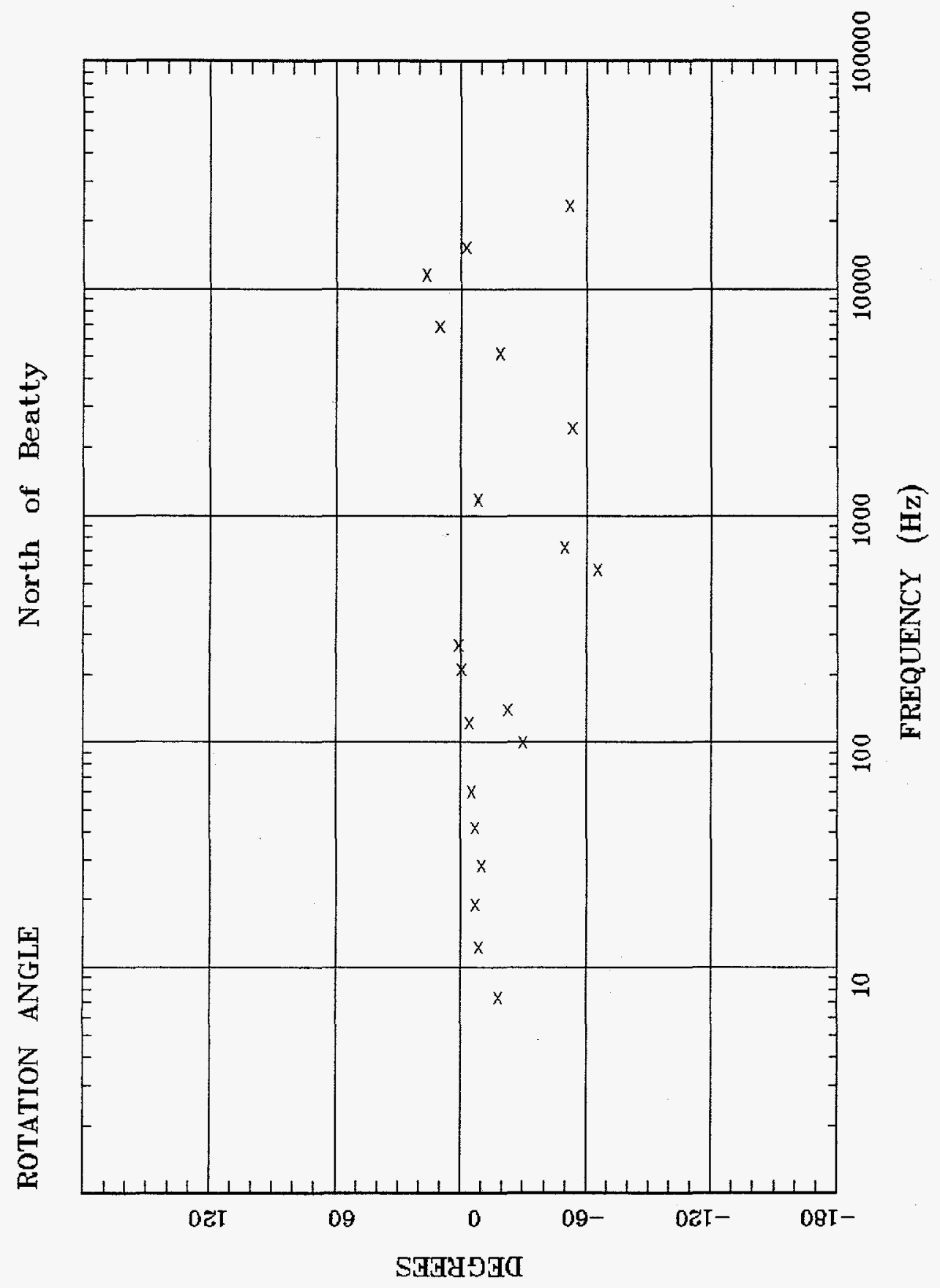

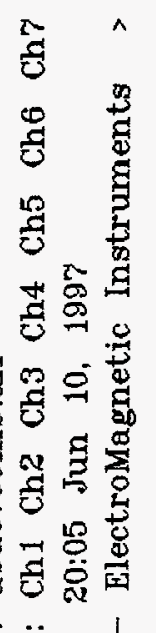

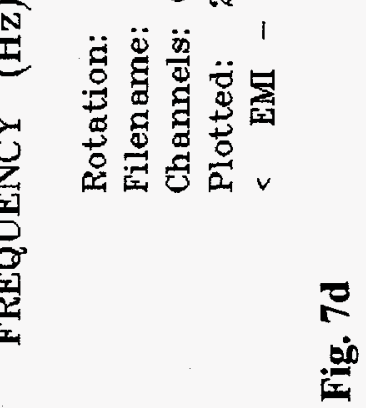

突

8

๑

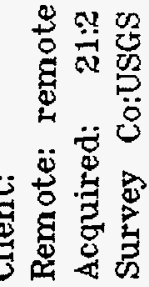

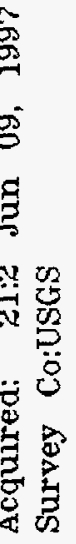




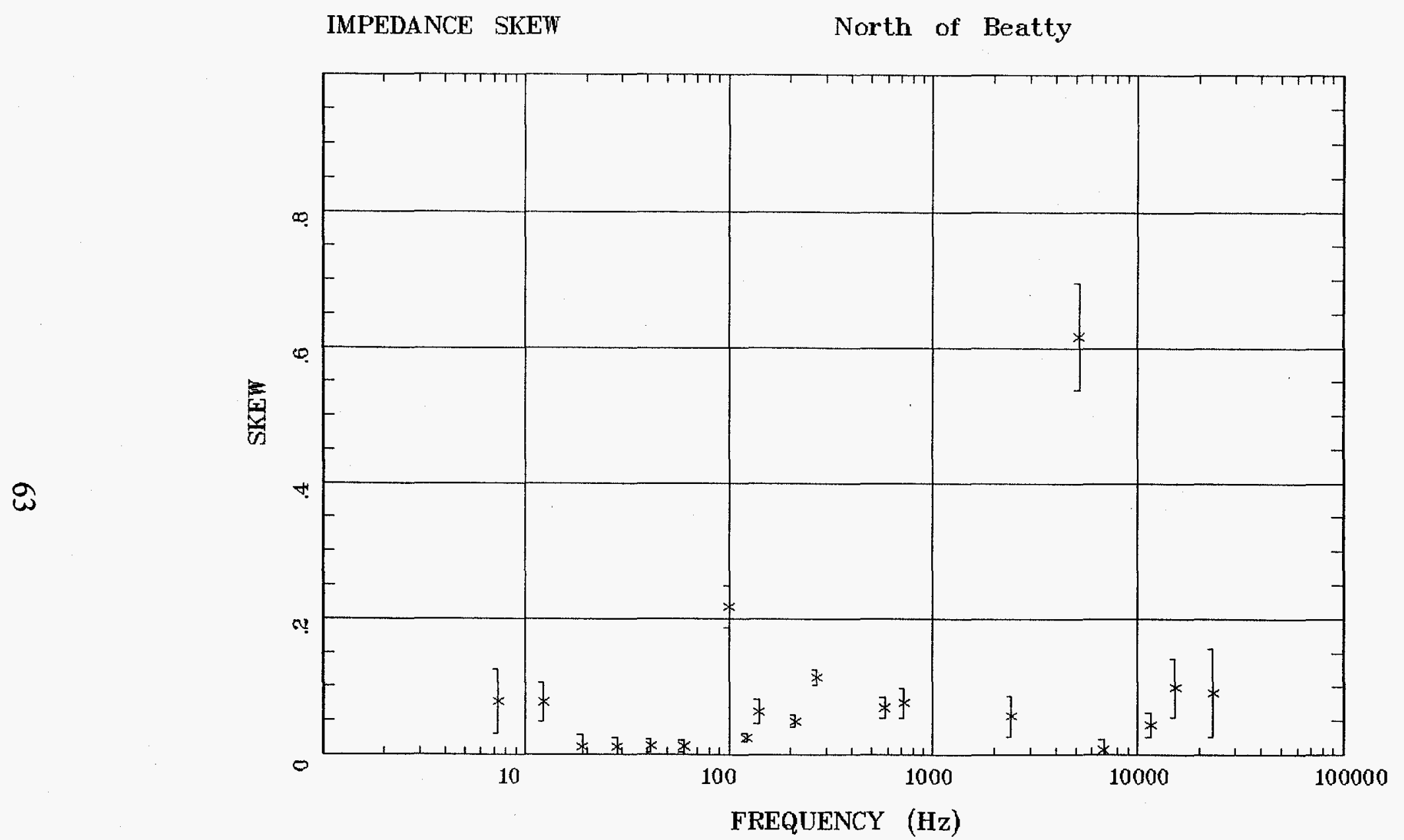

Client:

Remote: remote $e$

Acquired: 21:2 Jun 09, 1997

Survey Co:USGS
Rotation:

Filename: aba07comb.all

Channels: Ch1 Ch2 Ch3 ch4 Ch5 Ch6 Ch7

Plotted: 20:05 Jun 10, 1997

< EMI - ElectroMagnetic Instruments >

Fig. 7e 


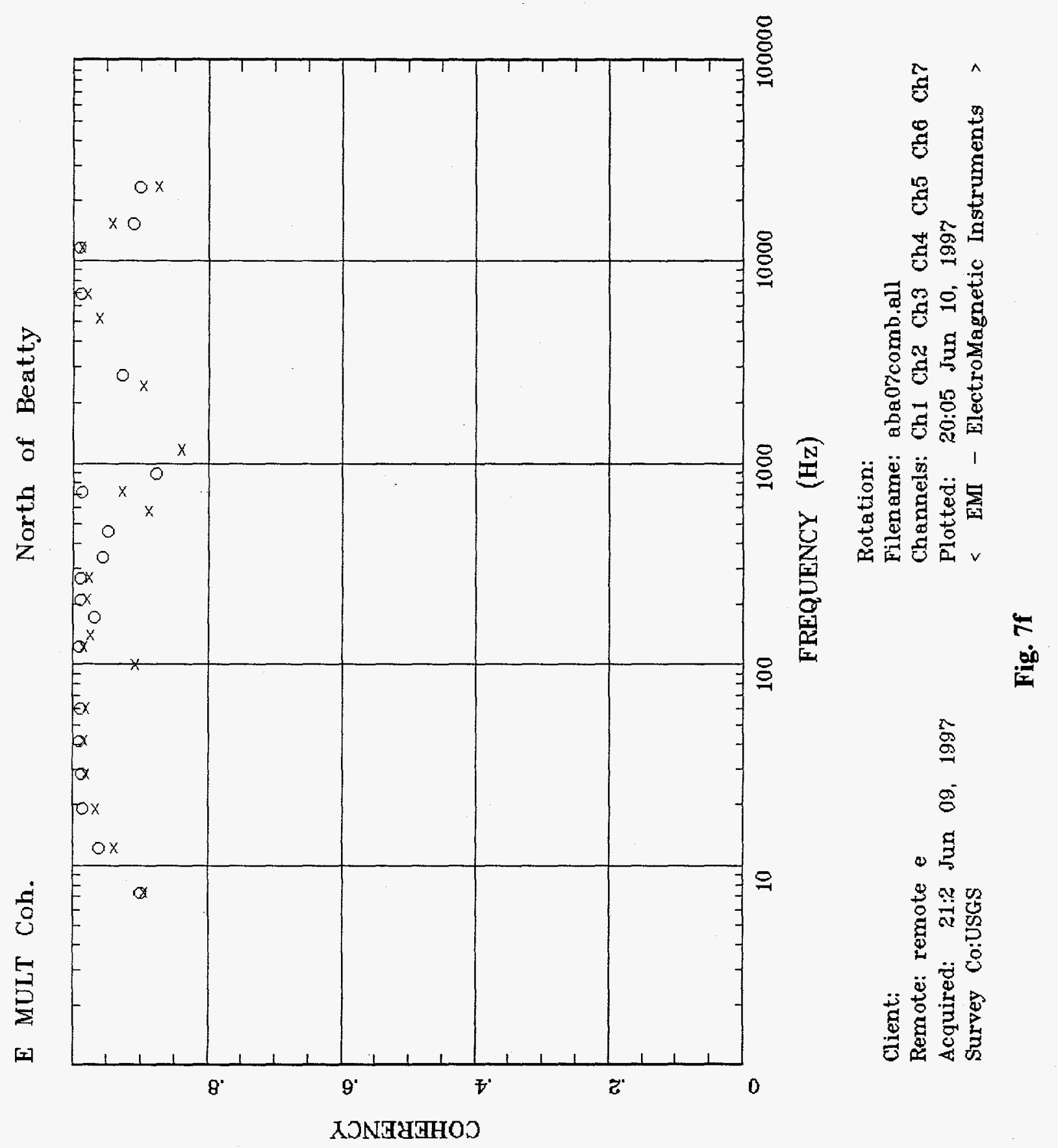


TIPPER MAGNITUDE

North of Beatty

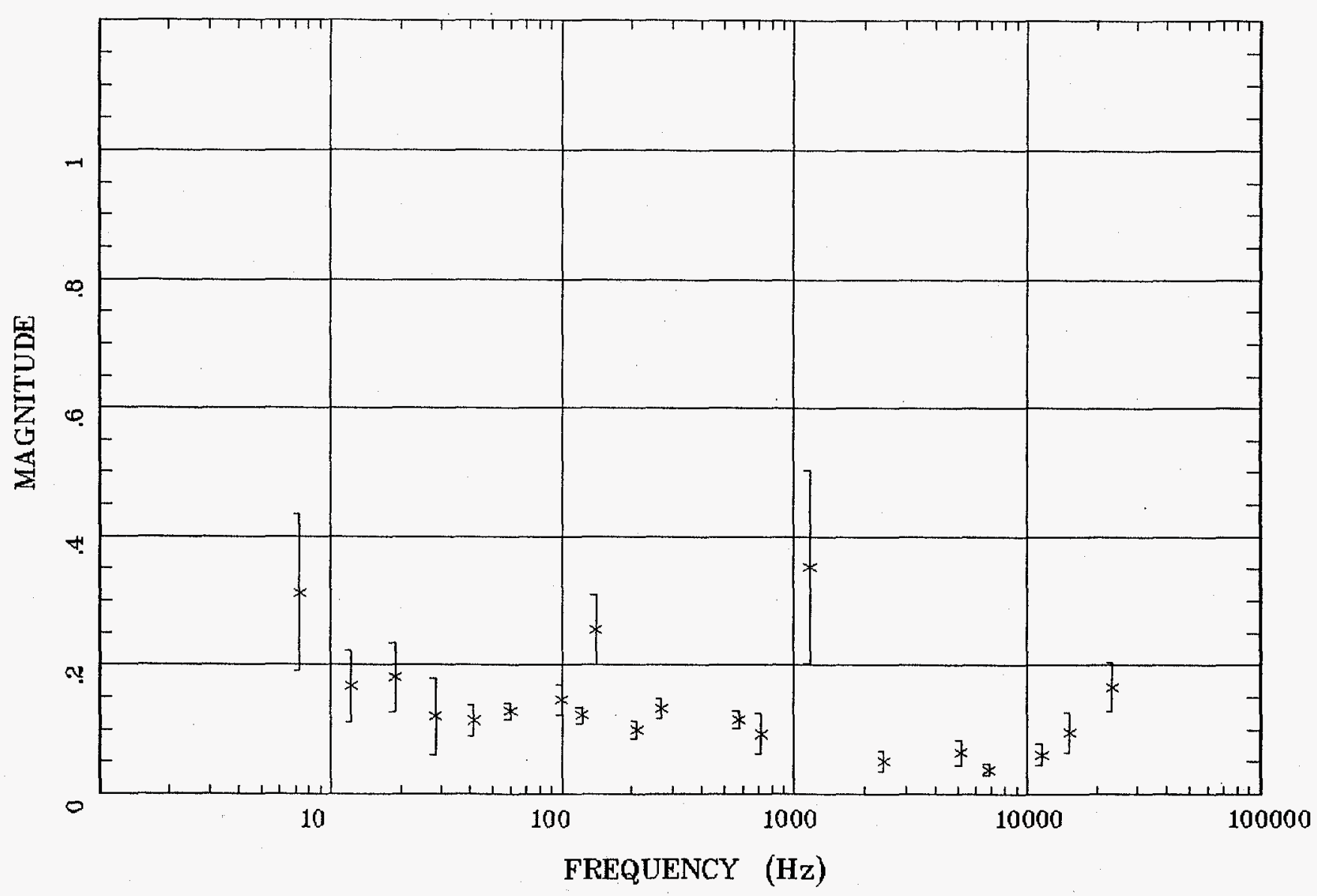

Client:

Rotation:

Remote: remote e

Acquired: 21:2 Jun 09, 1997

Filename: aba07comb.all

Channels: Ch1 Ch2 Ch3 Ch4 Ch5 Ch6 Ch7

Plotted: 20:05 Jun 10, 1997

Survey Co:USGS

< EMI - ElectroMagnetic Instruments >

Fig. $7 g$ 

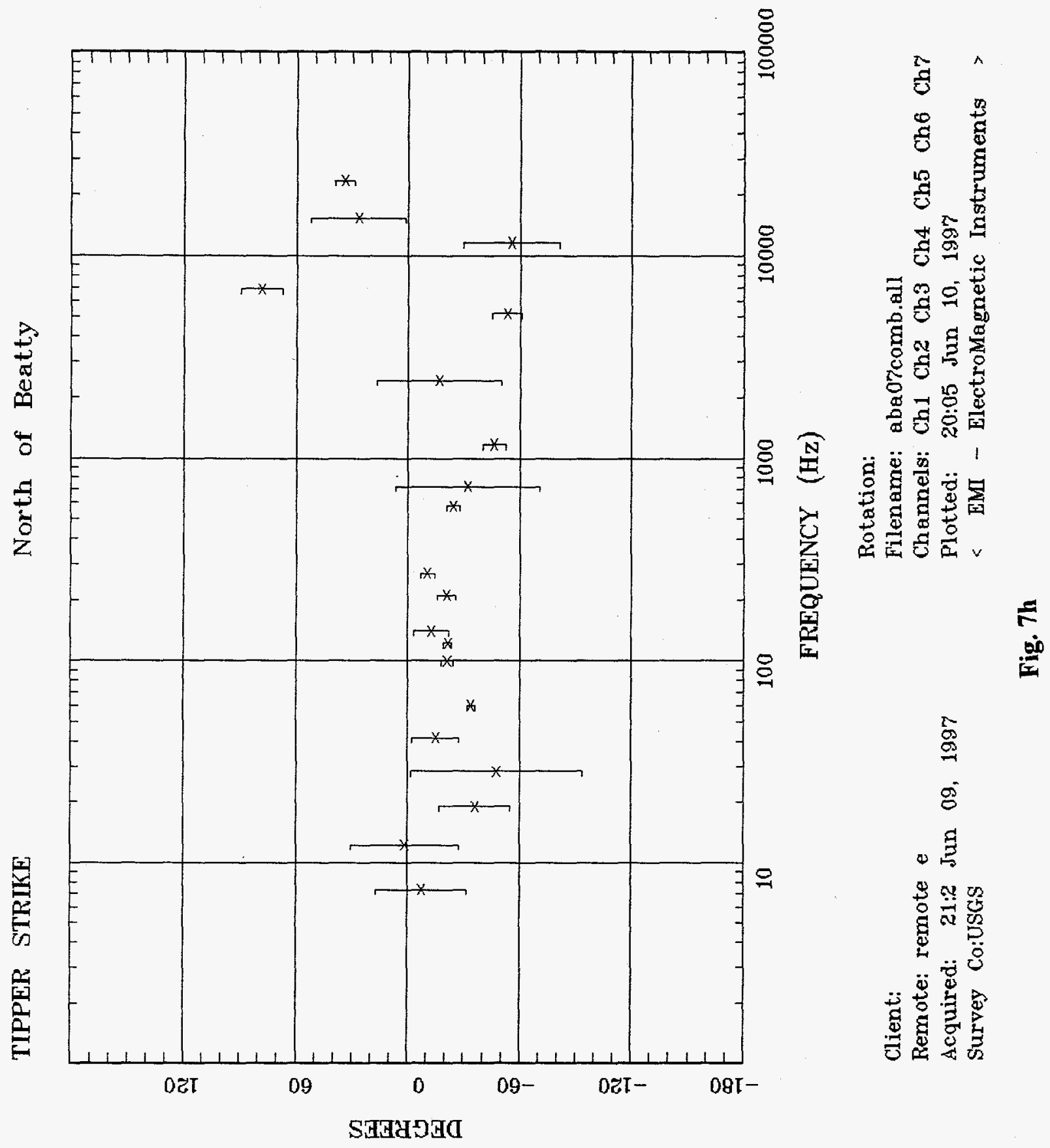


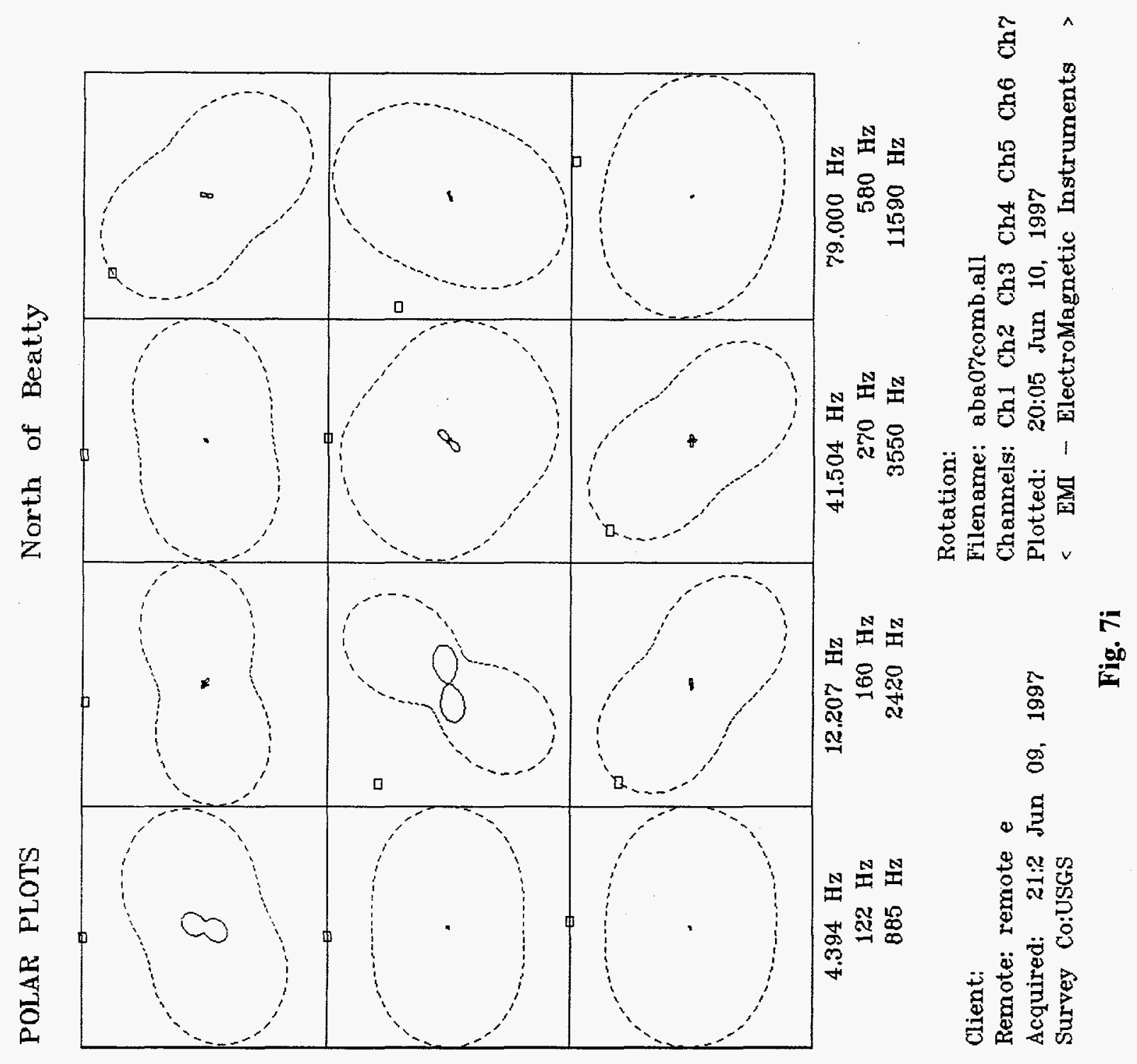


Resistivity (Bostick Inv.)

2

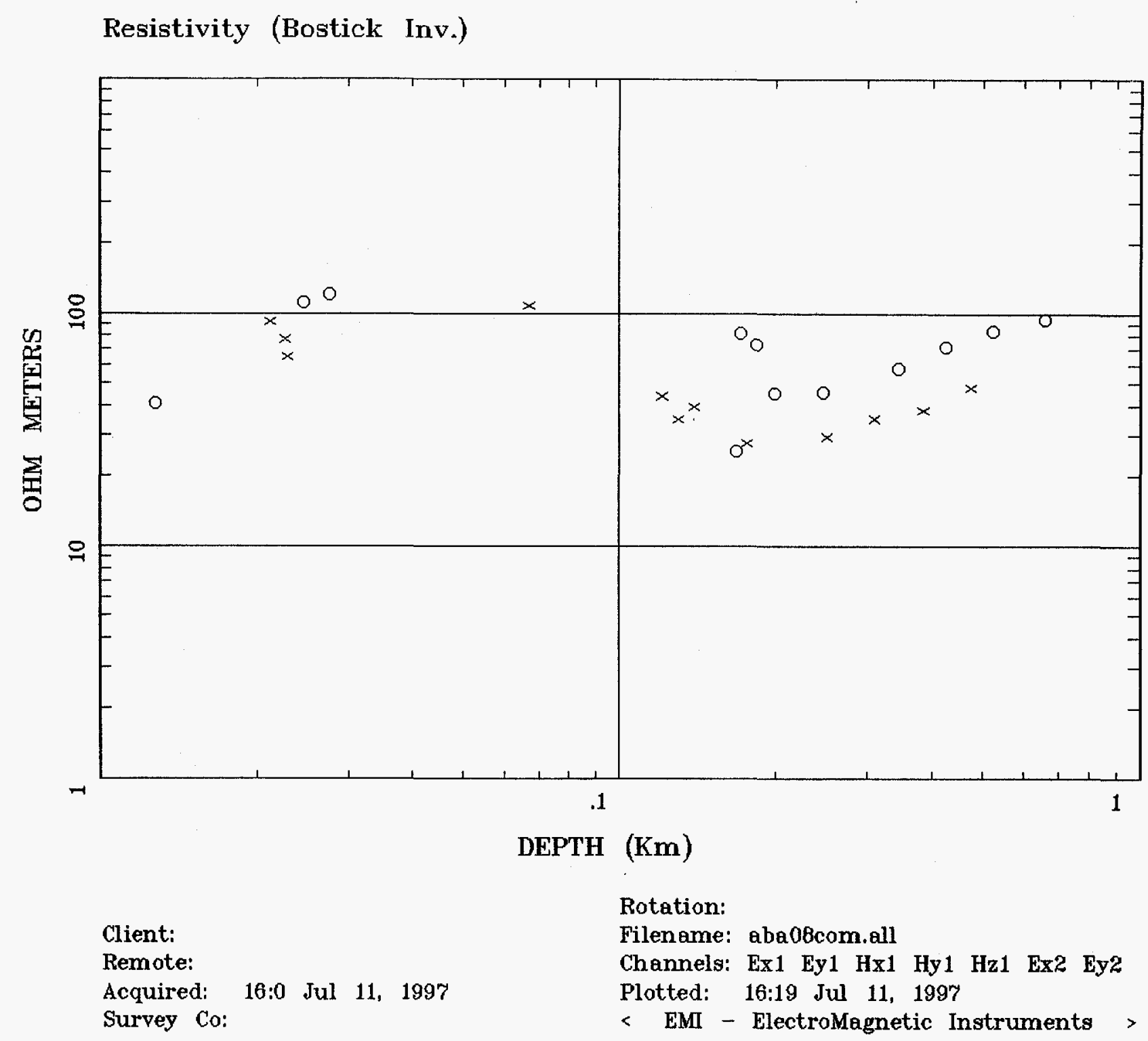

Fig. 8a 


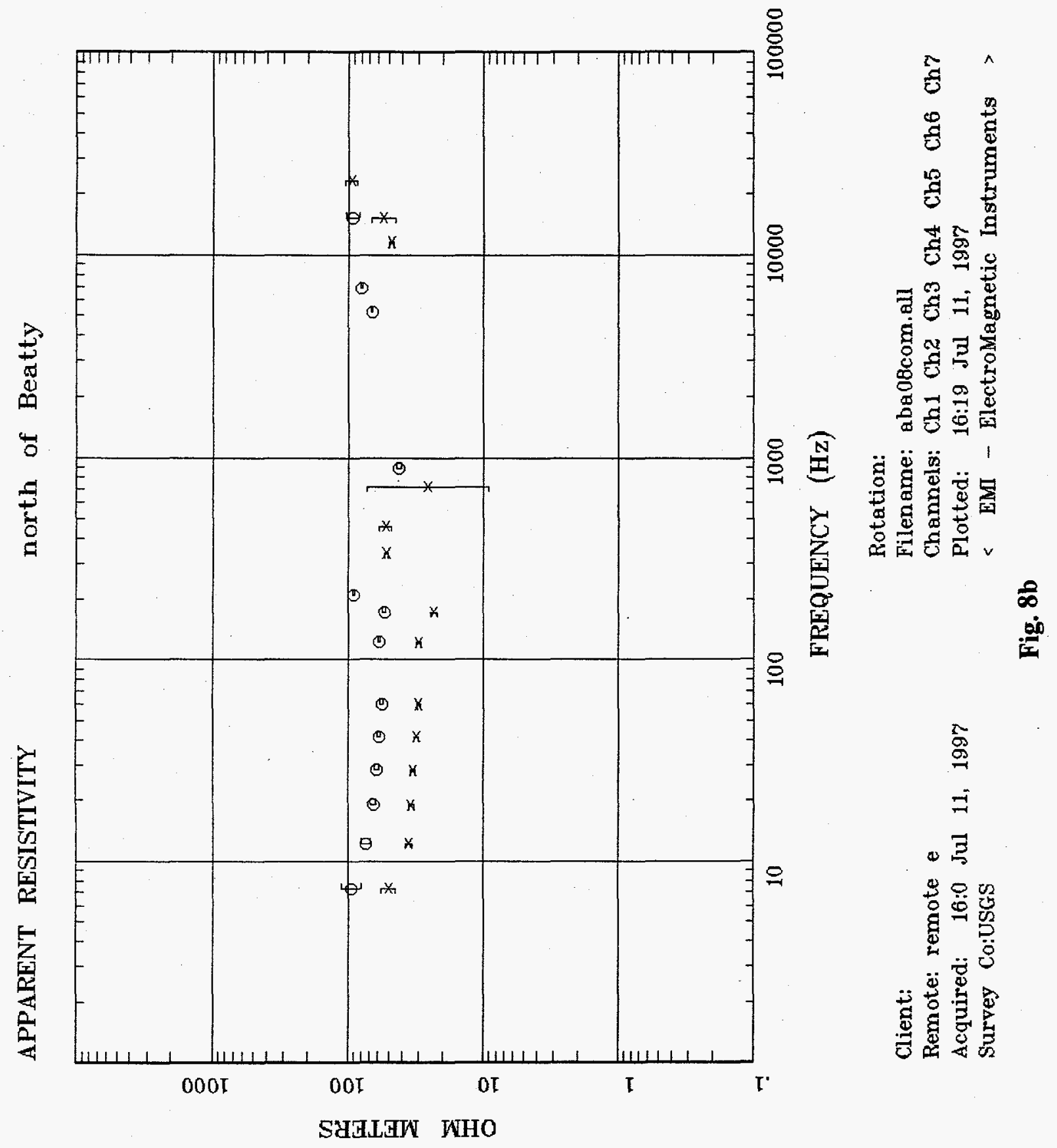


IMPEDANCE PHASE

north of Beatty

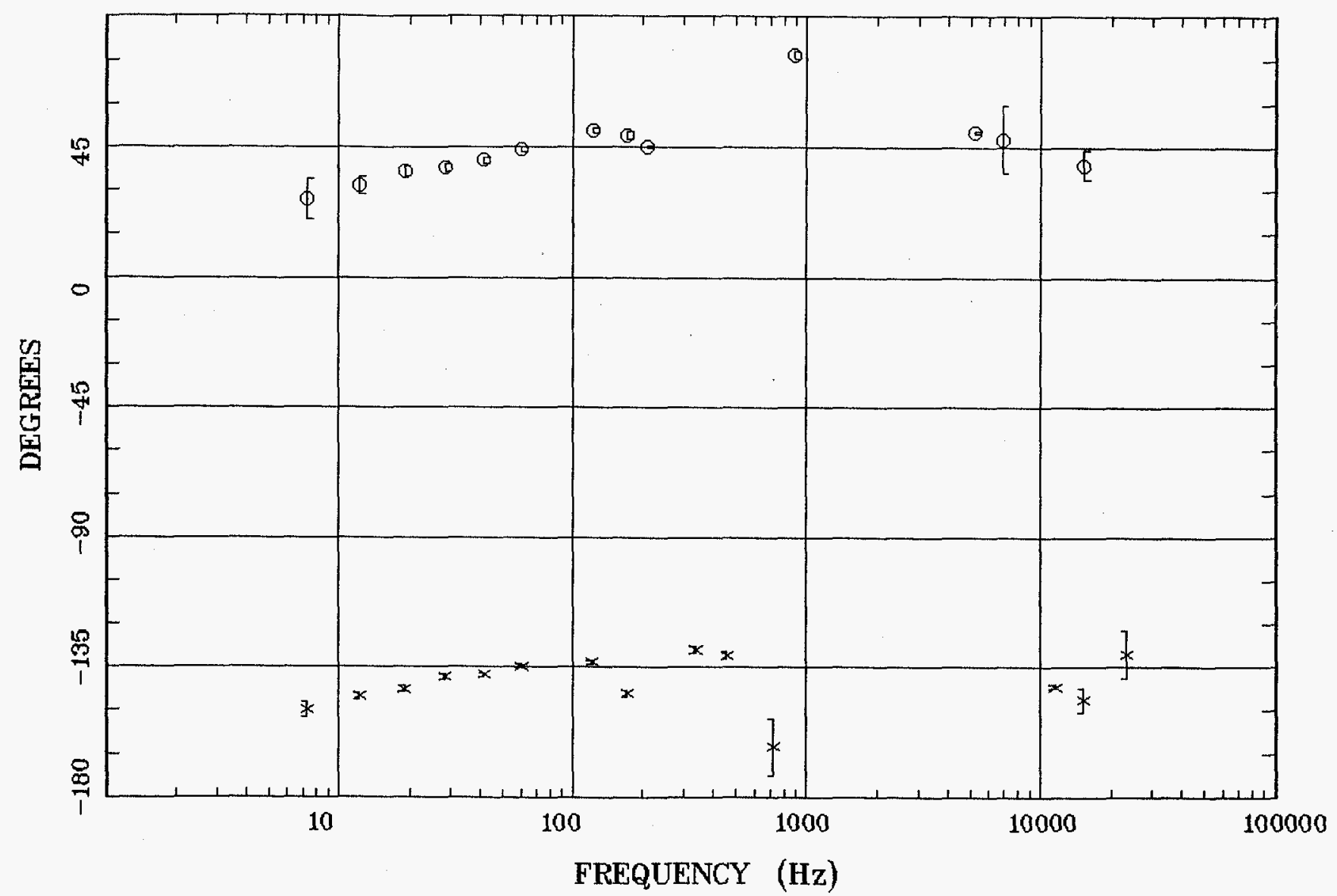

Client:

Remote: remote e

Acquired: 16:0 Jul 11, 1997

Rotation

Filename: aba08com.all

Channels: Ch1 Ch2 Ch3 Ch4 Ch5 Ch6 Ch7

Platted: 16:19 Jul 11, 1997

Survey Co:USGS

< EMI - ElectroMagnetic Instruments >

Fig. 8c 

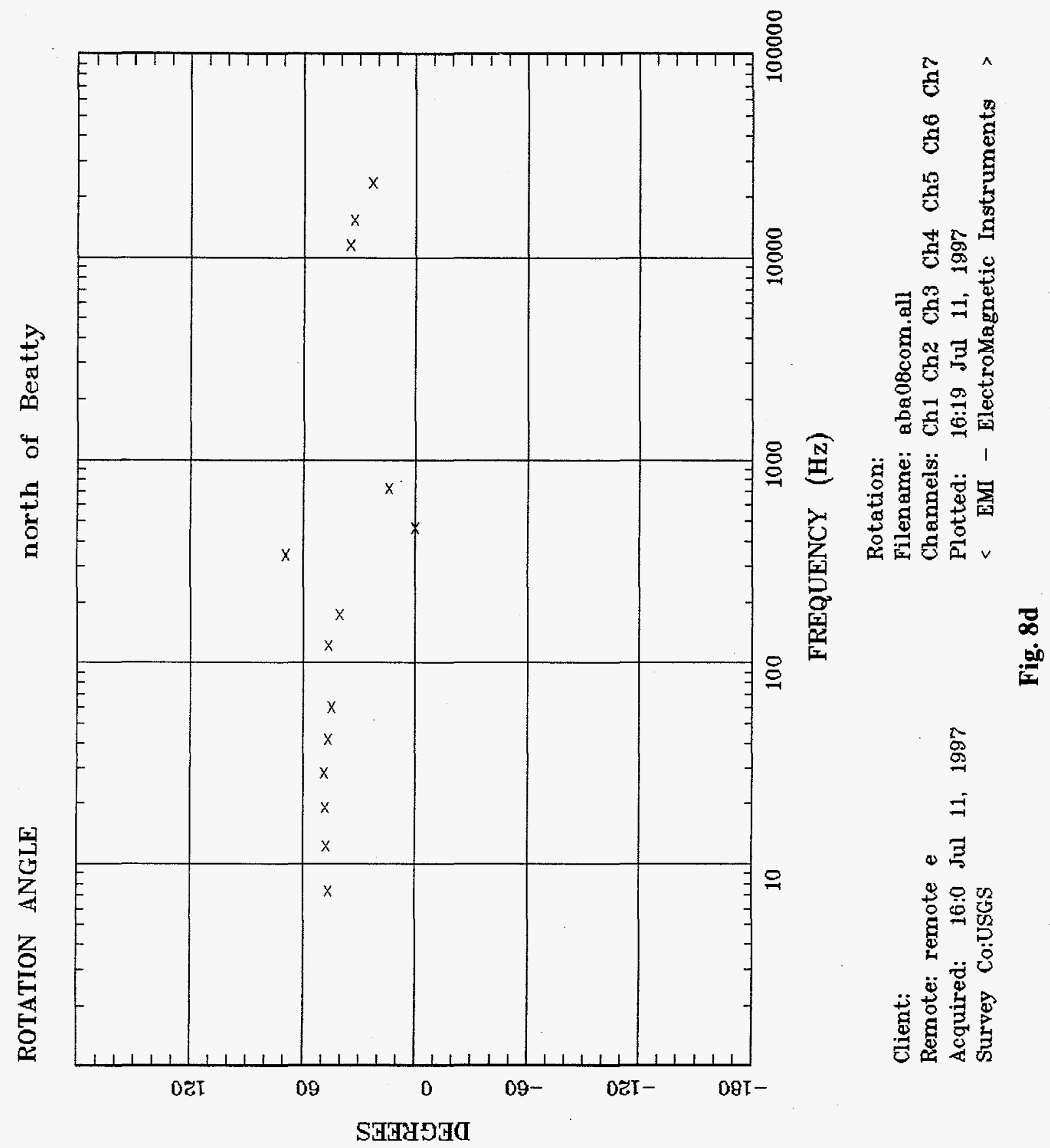


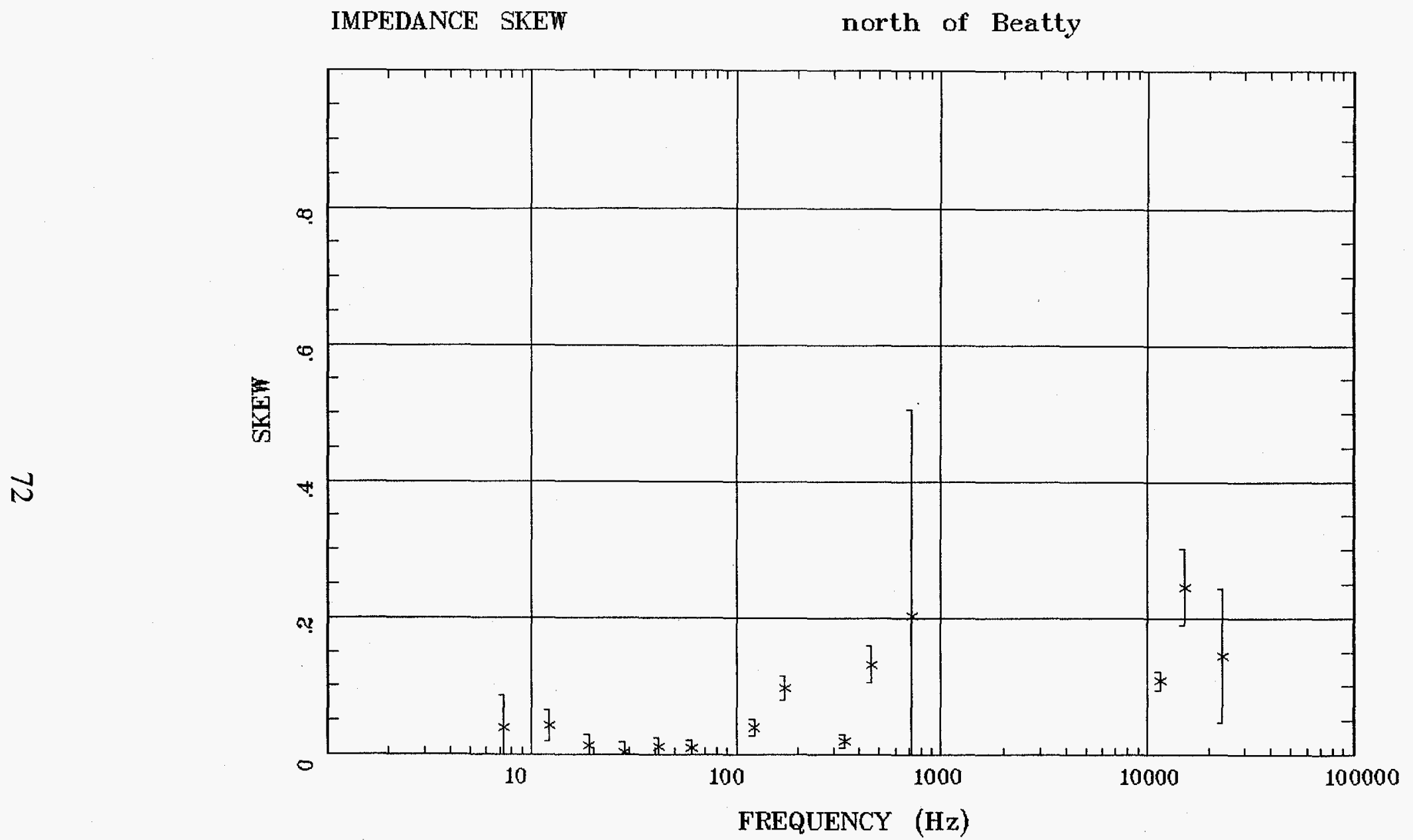

Client:

Remote: remote e

Acquired: 16:0 Jul 11, 1997

Survey Co:USGS
Rotation:

Filename: aba08com.all

Channels: Ch1 Ch2 Ch3 Ch4 Ch5 Ch6 Ch7

Plotted: 16:19 Jul 11, 1997

< EMI - ElectroMagnetic Instruments >

Fig. 8e 


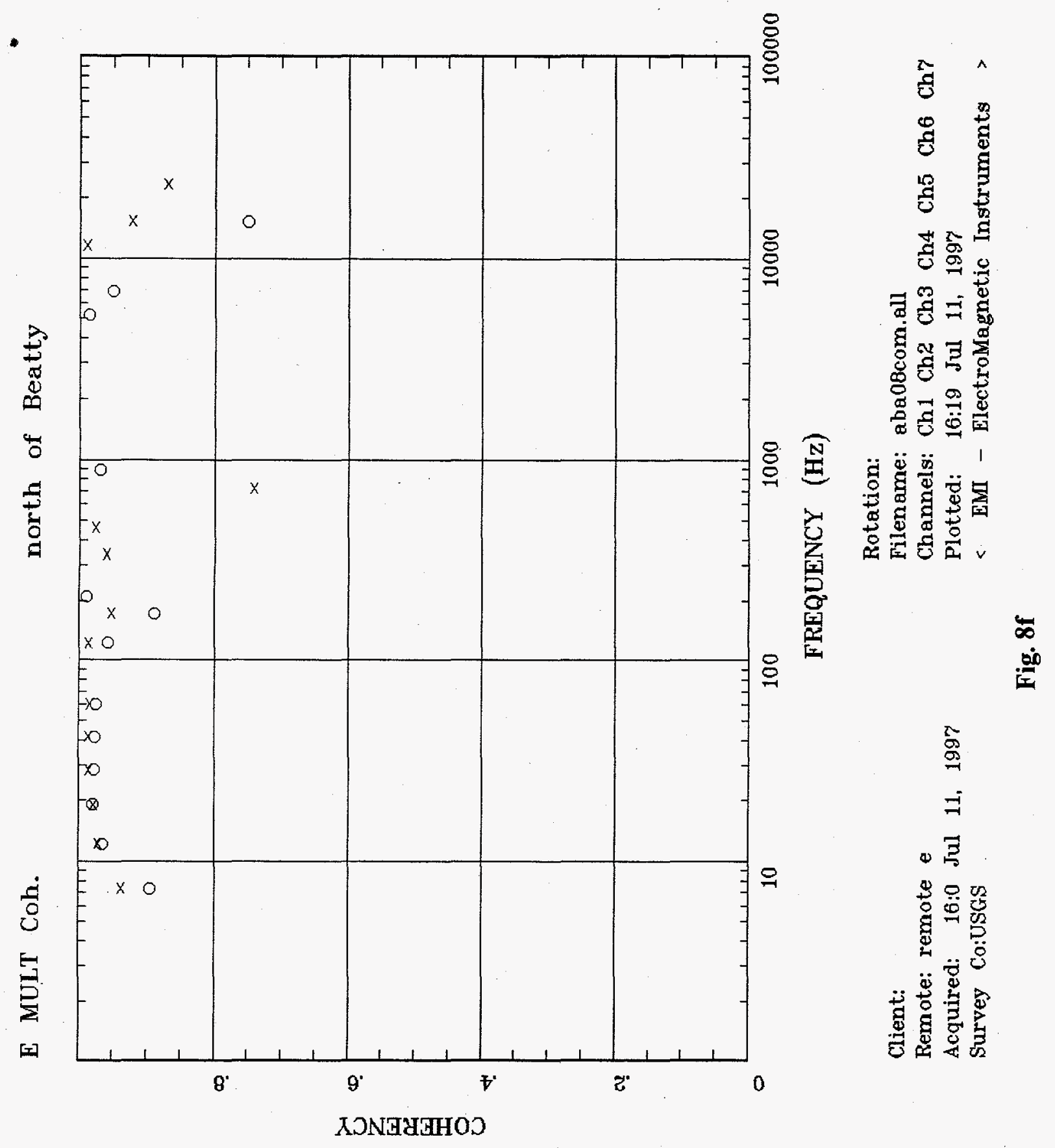




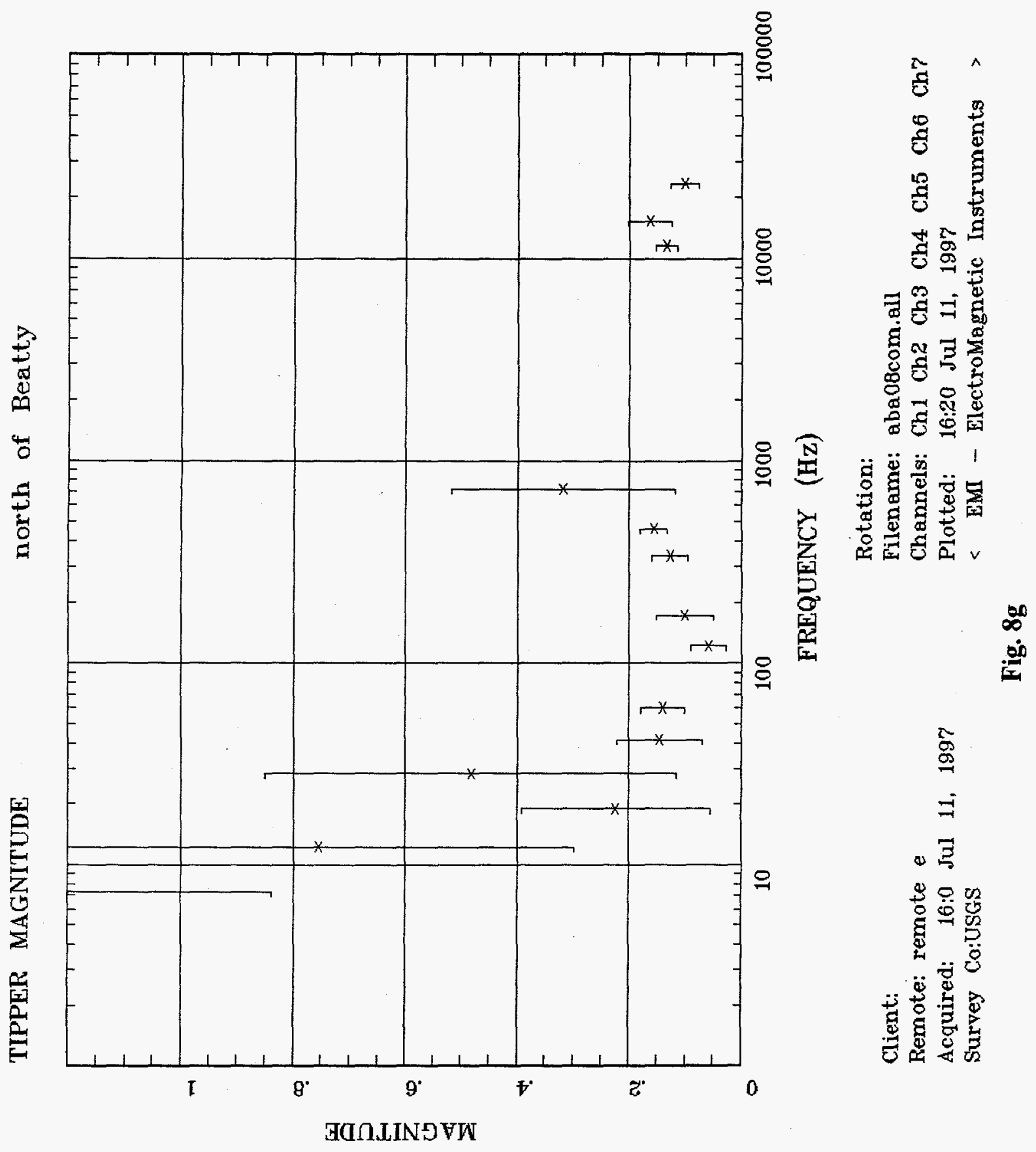





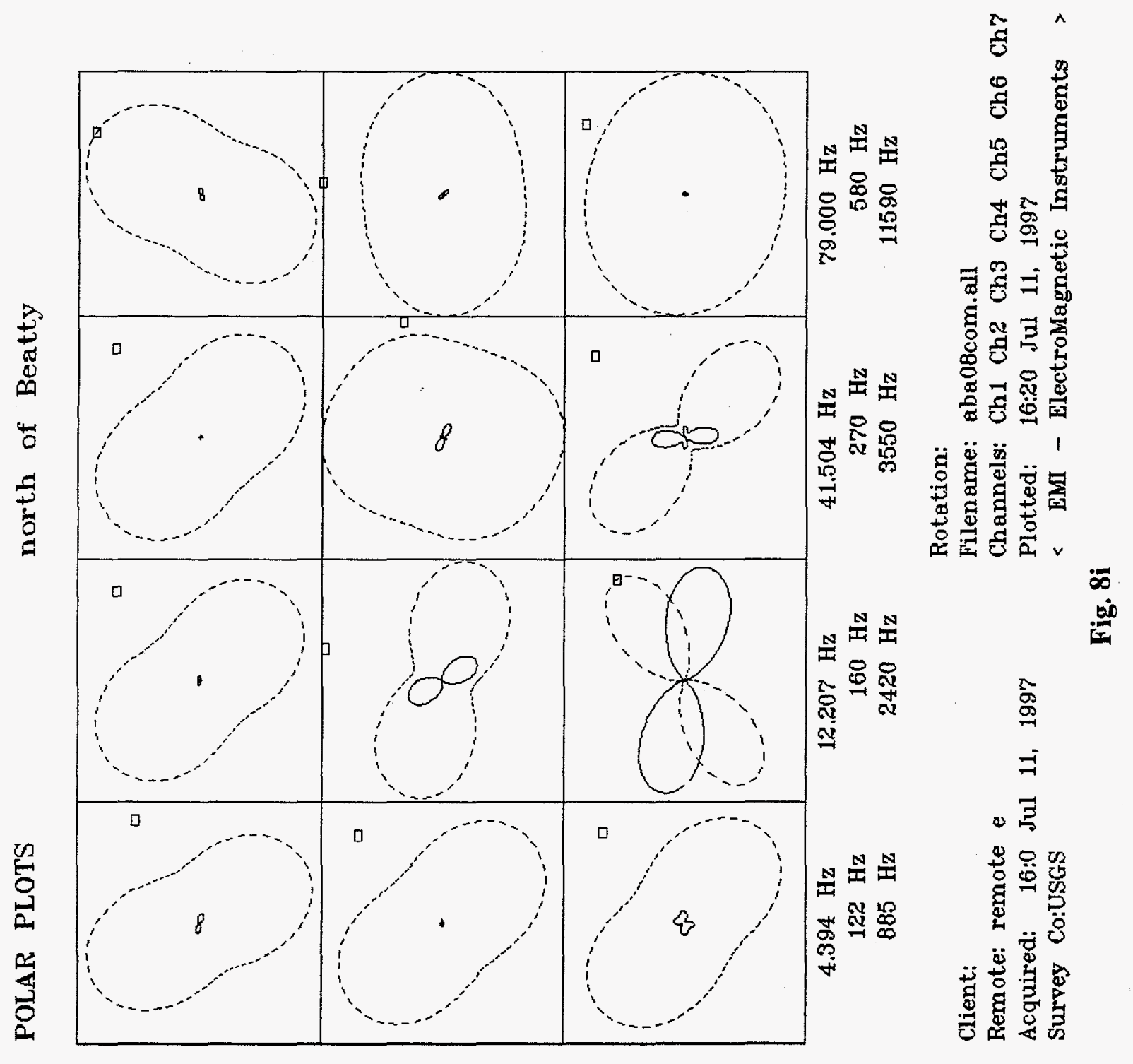




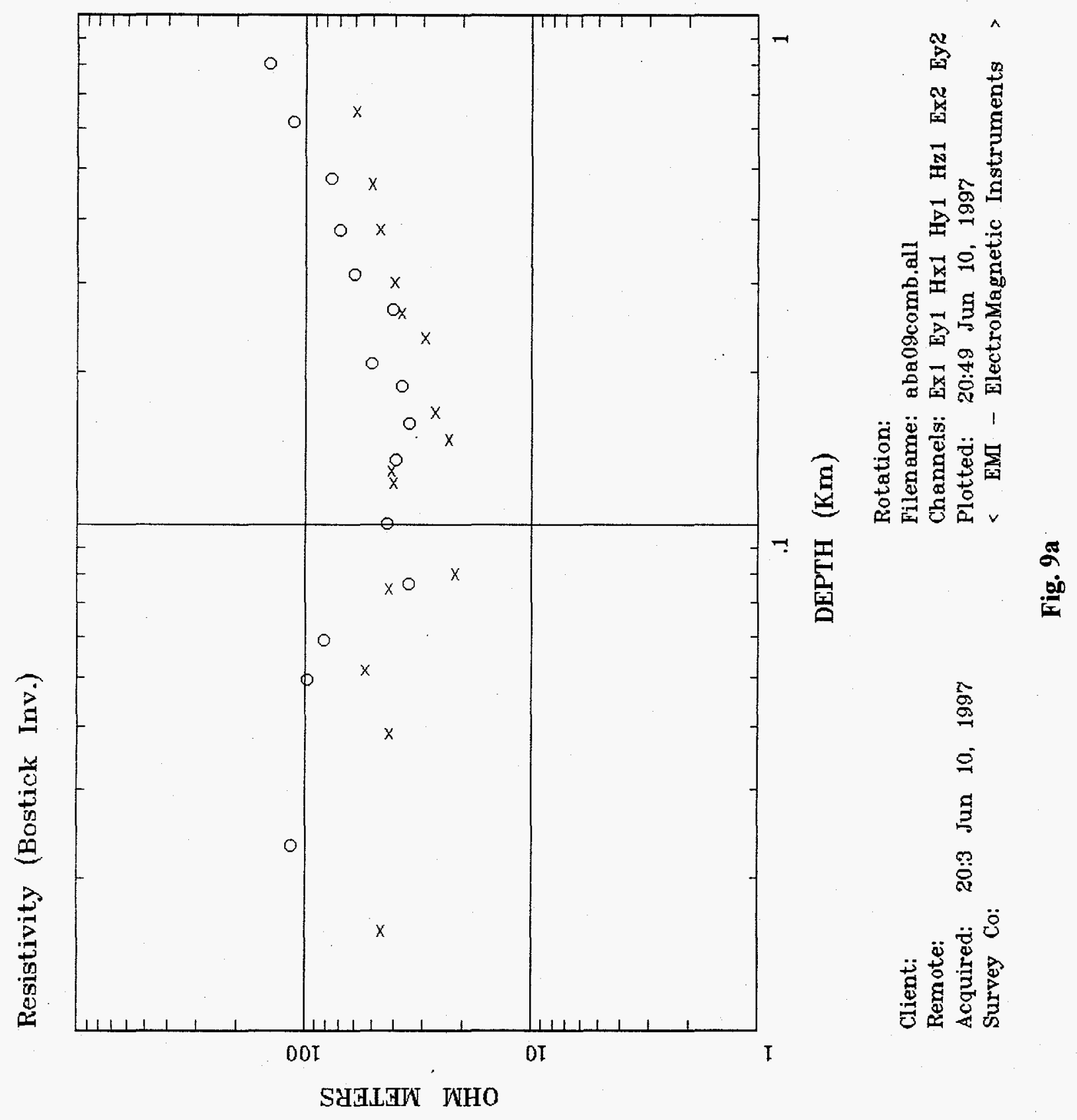




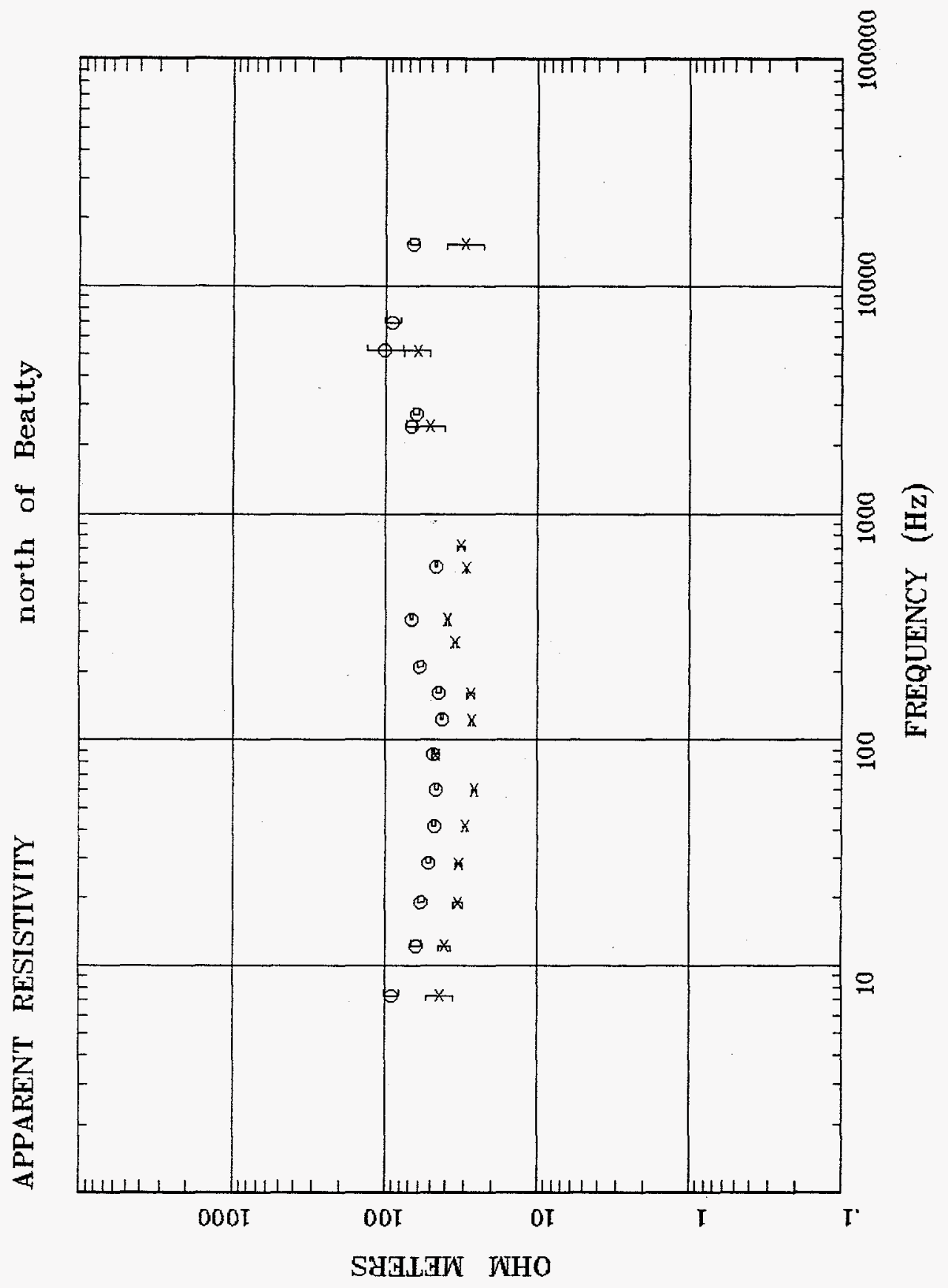

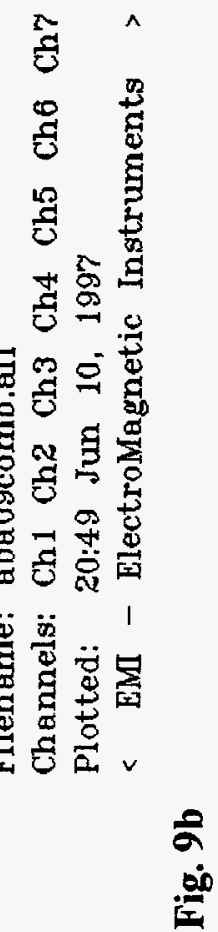

$\frac{\pi}{2}$

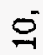

๑)

\$ั

के

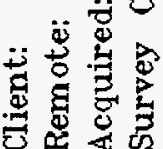

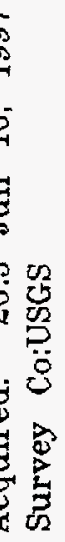

0

SมHLIT NHO 


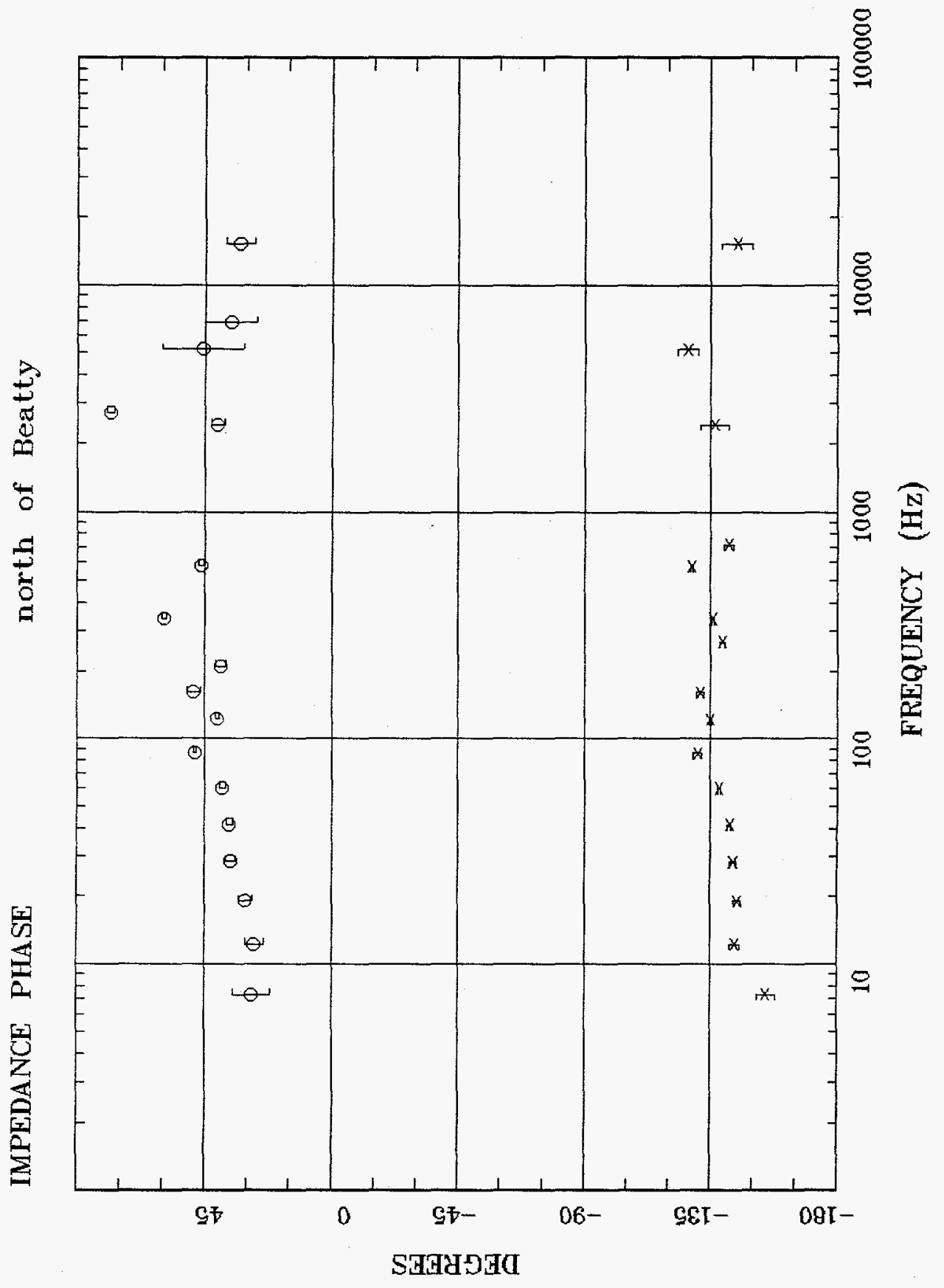

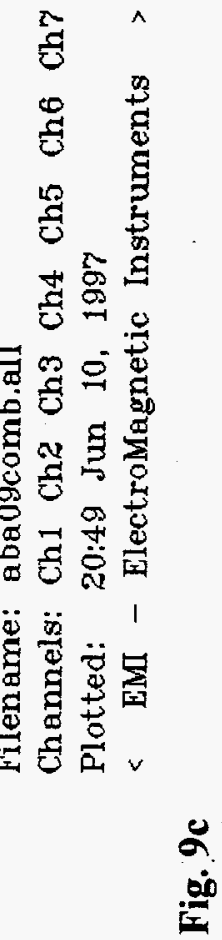

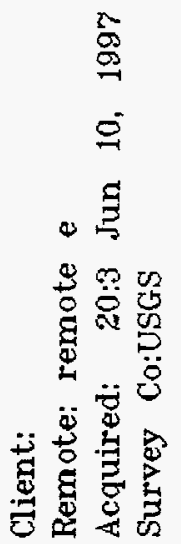


ROTATION ANGLE north of Beatty

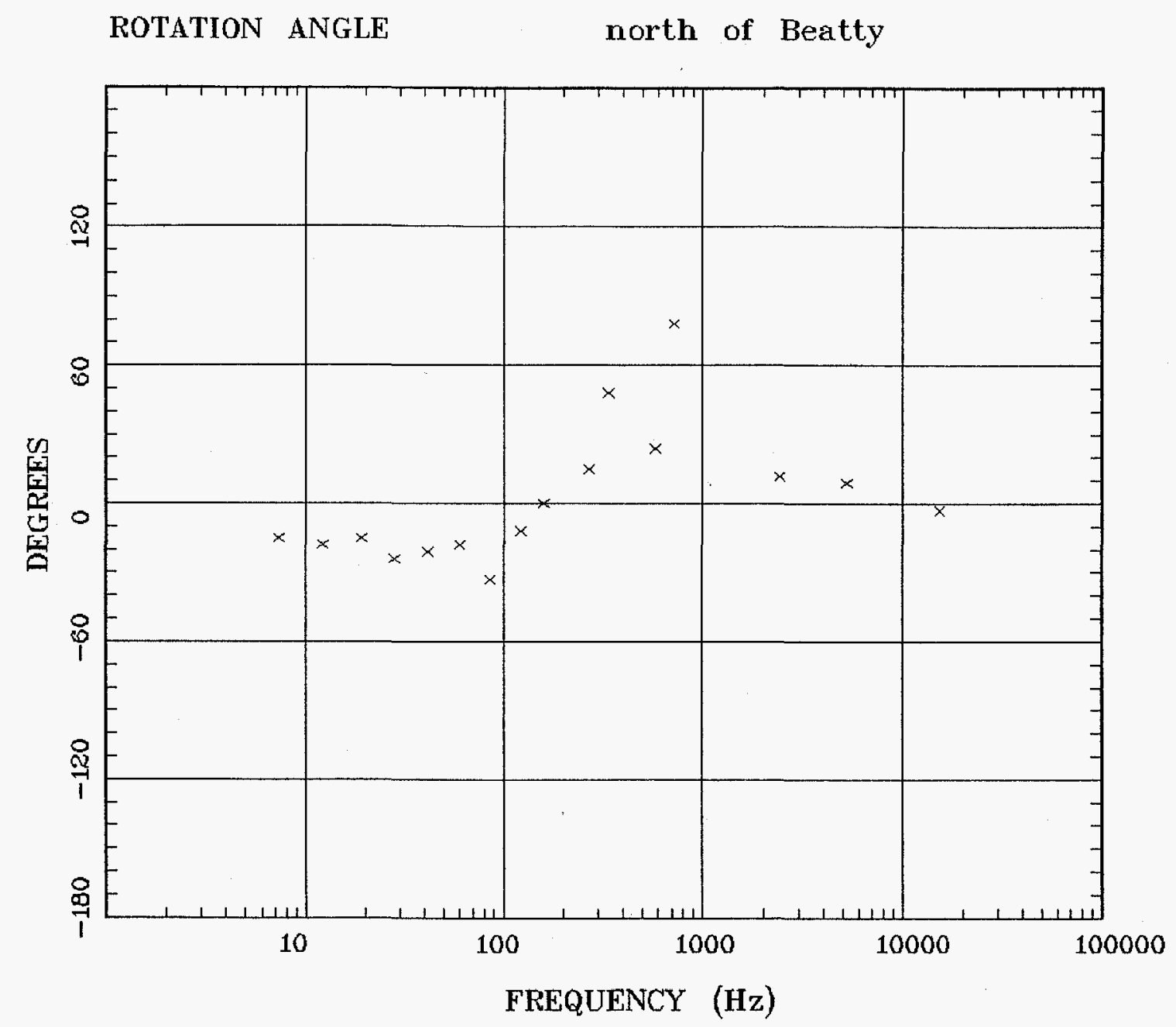

Client:

Remote: remote e

Acquired: 20:3 Jun 10, 1997 Survey Co:USGS
Rotation:

Filename: aba09comb.all

Channels: Ch1 Ch2 Ch3 Ch4 Ch5 Ch6

Plotted: 20:49 Jun 10, 1997

$<$ EMI - ElectroMagnetic Instruments

Fig. 9d 


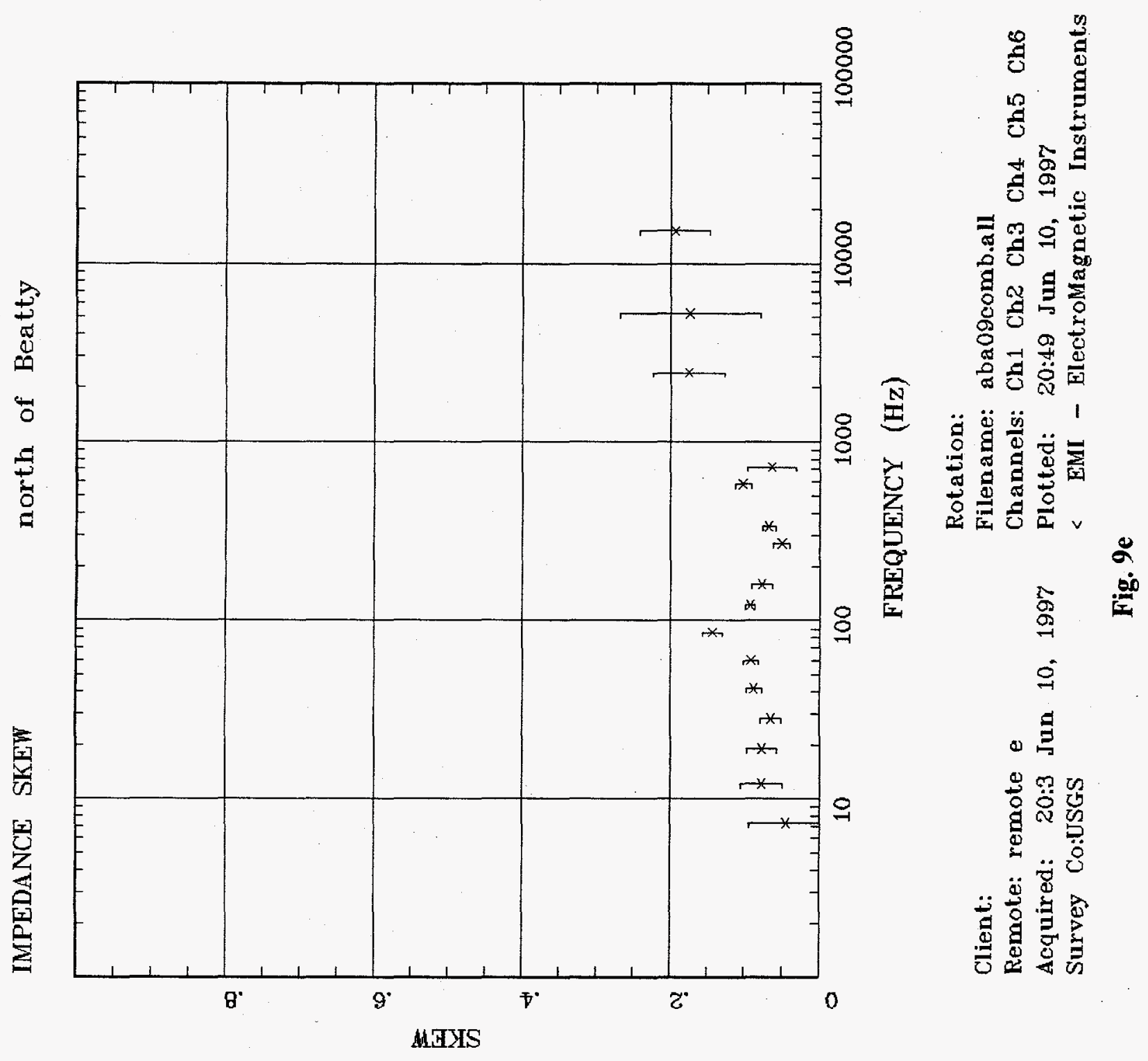




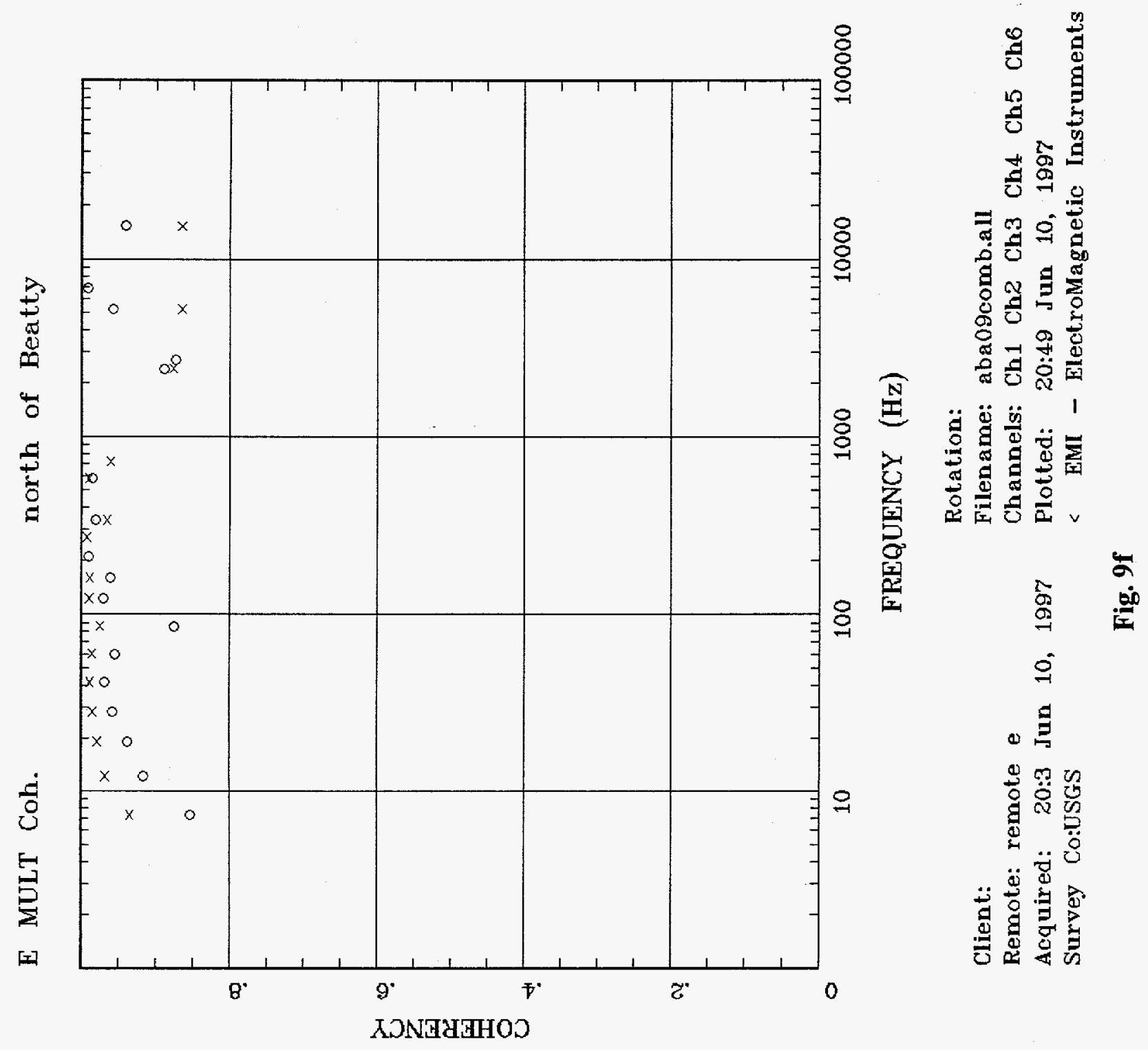


TIPPER MAGNITUDE

north of Beatty

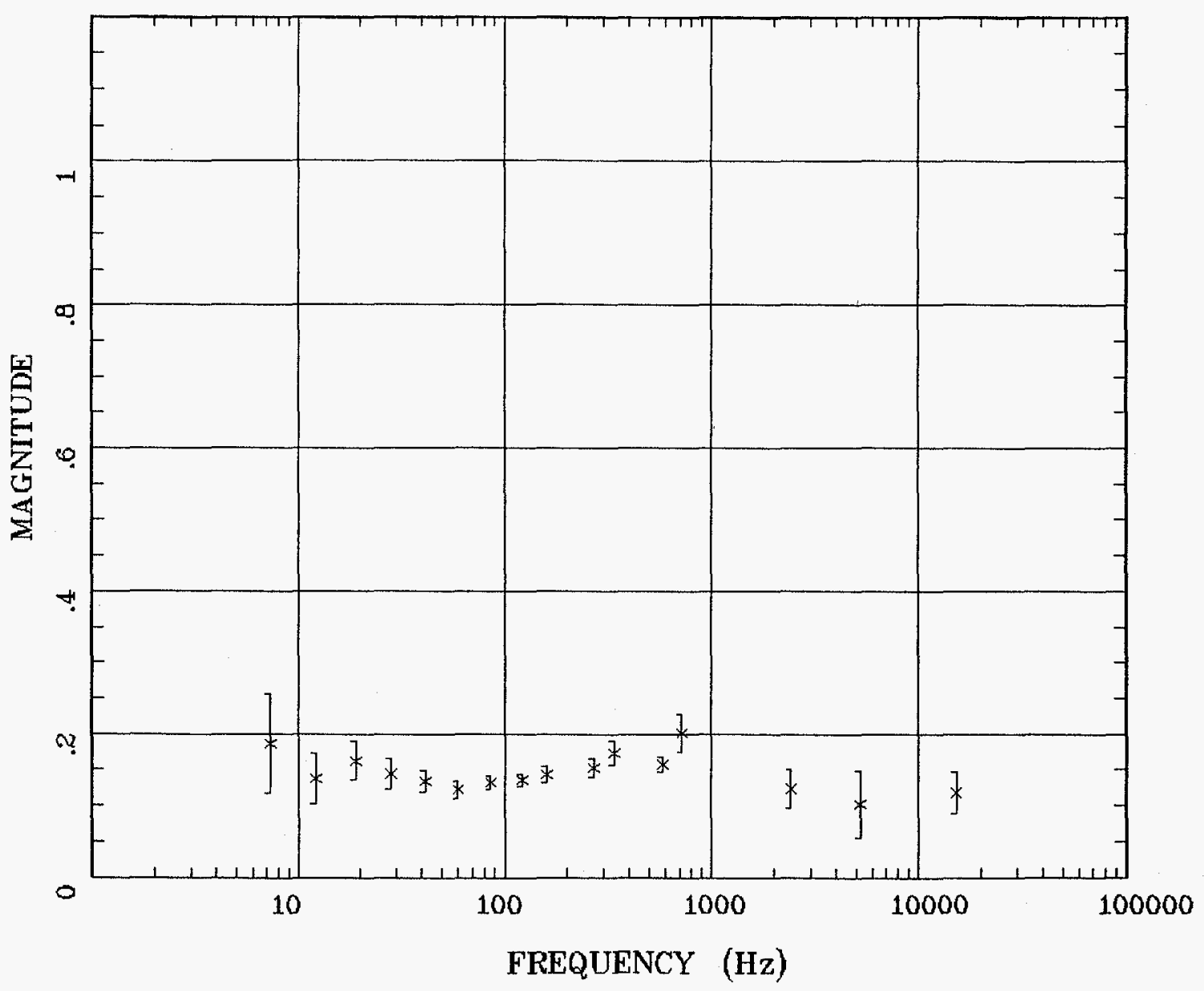

\section{Client:}

Remote: remote e

Acquired: 20:3 Jun 10, 1997 Survey Co:USGS
Rotation:

Filename: aba09comb.all

Channels: Ch1 Ch2 Ch3 Ch4 Ch5 Ch6

Plotted: 20:49 Jun 10, 1997

$<$ EMI - ElectroMagnetic Instruments

Fig. 9g 
TIPPER STRIKE

north of Beatty

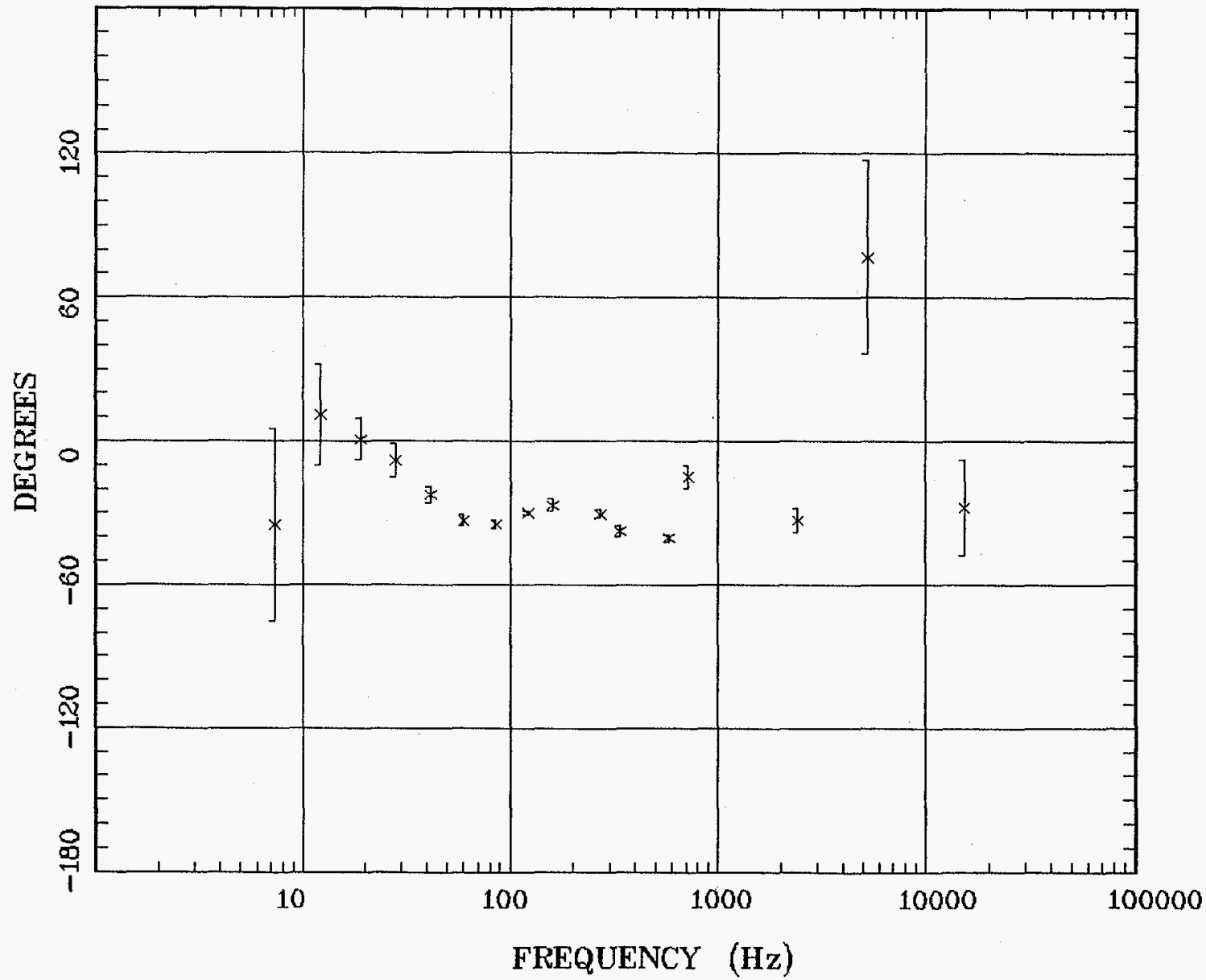

Client:

Remote: remote

Acquired: 20:3 Jun 10, 199 y

Survey Co:USGS
Rotation:

Filename: aba09comb.all

Channels: Ch1 Ch2 Ch3 Ch4 Ch5 Ch6

Plotted: 20:50 Jun 10, 1997

< EMI - ElectroMagnetic Instruments

Fig. 9h 


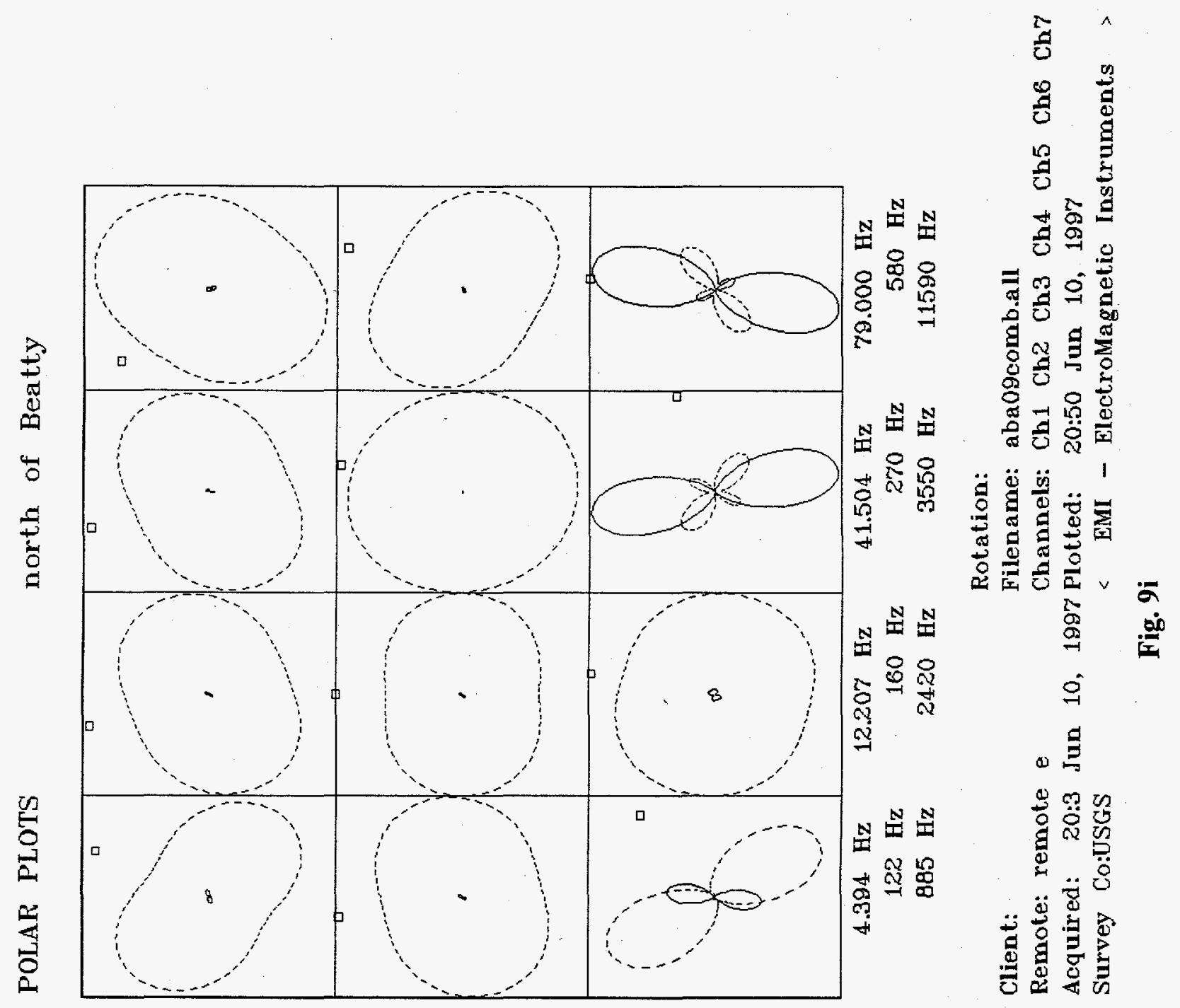


Resistivity (Bostick Inv.)

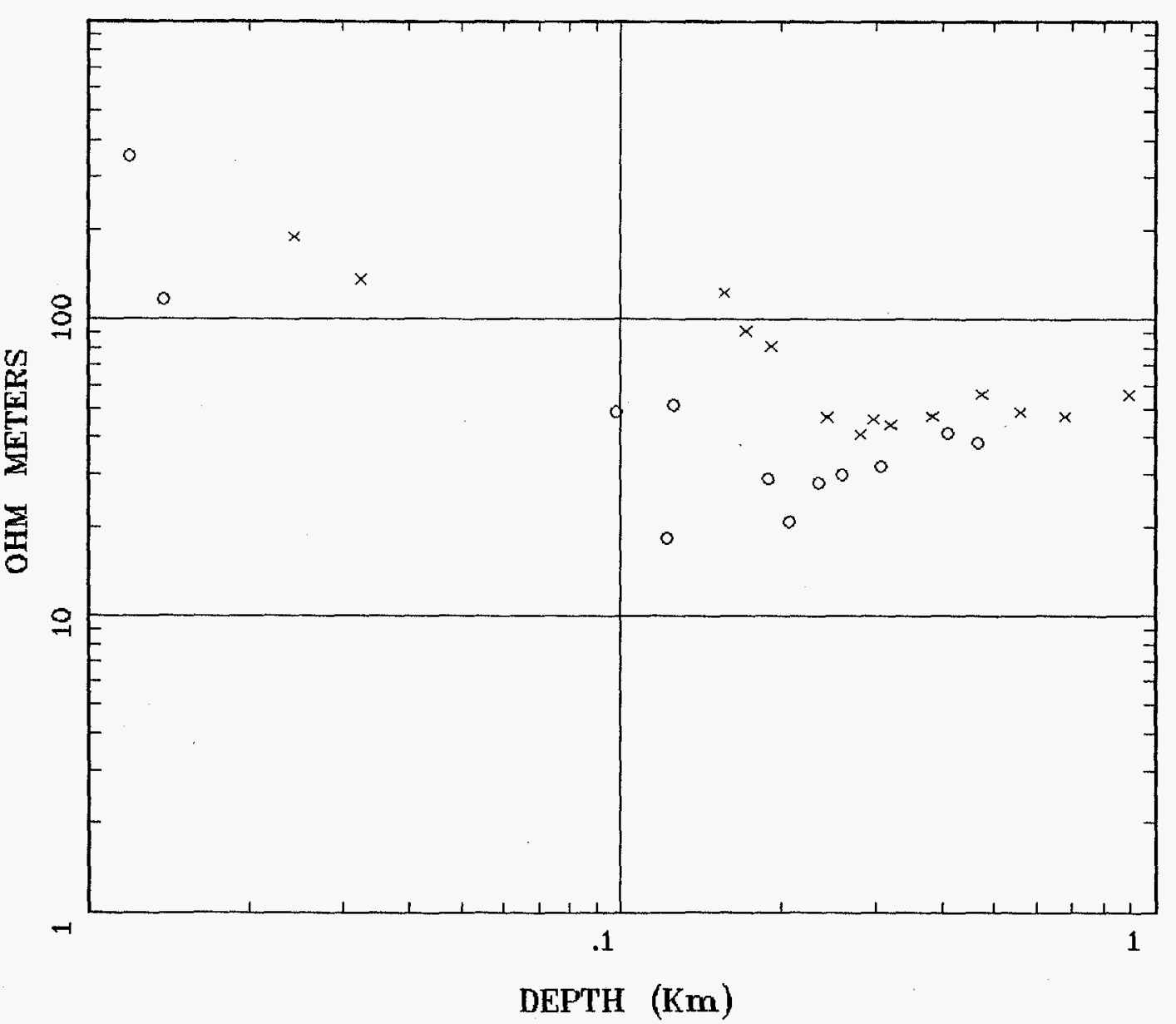

Client:

\section{Remote:}

Acquired: 14:1 Sep 05, 1997 Survey Co:
Rotation:

Filename: bba10com.all

Channels: Ex1 Ey1 $\mathrm{Hx} 1 \mathrm{Hy} 1 \mathrm{~Hz} 1 \mathrm{Ex} 2$

Plotted: 10:44 Sep 17, 1997

< EMI - ElectroMagnetic Instruments

Fig. 10a 


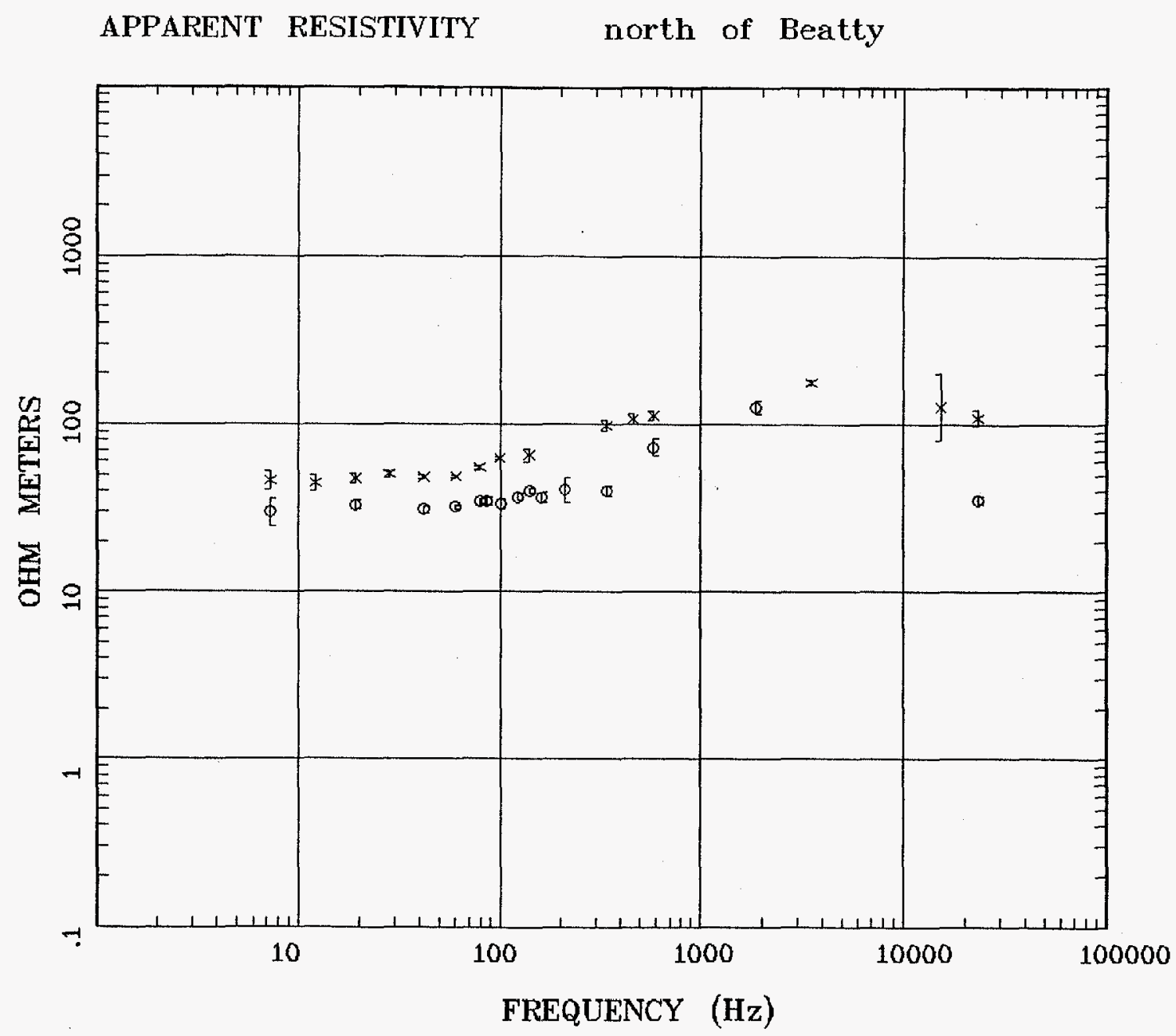

Client:

Remote: remote e

Acquired: 14:1 Sep 05, 1997 Survey Co:USGS
Rotation:

Filename: bba10com.all

Channels: Ch1 Ch2 Ch3 Ch4 Ch5 Ch6

Plotted: 10:44 Sep 17, 1997

$<$ EMI - ElectroMagnetic Instruments

Fig. 10b 
IMPEDANCE PHASE

north of Beatty

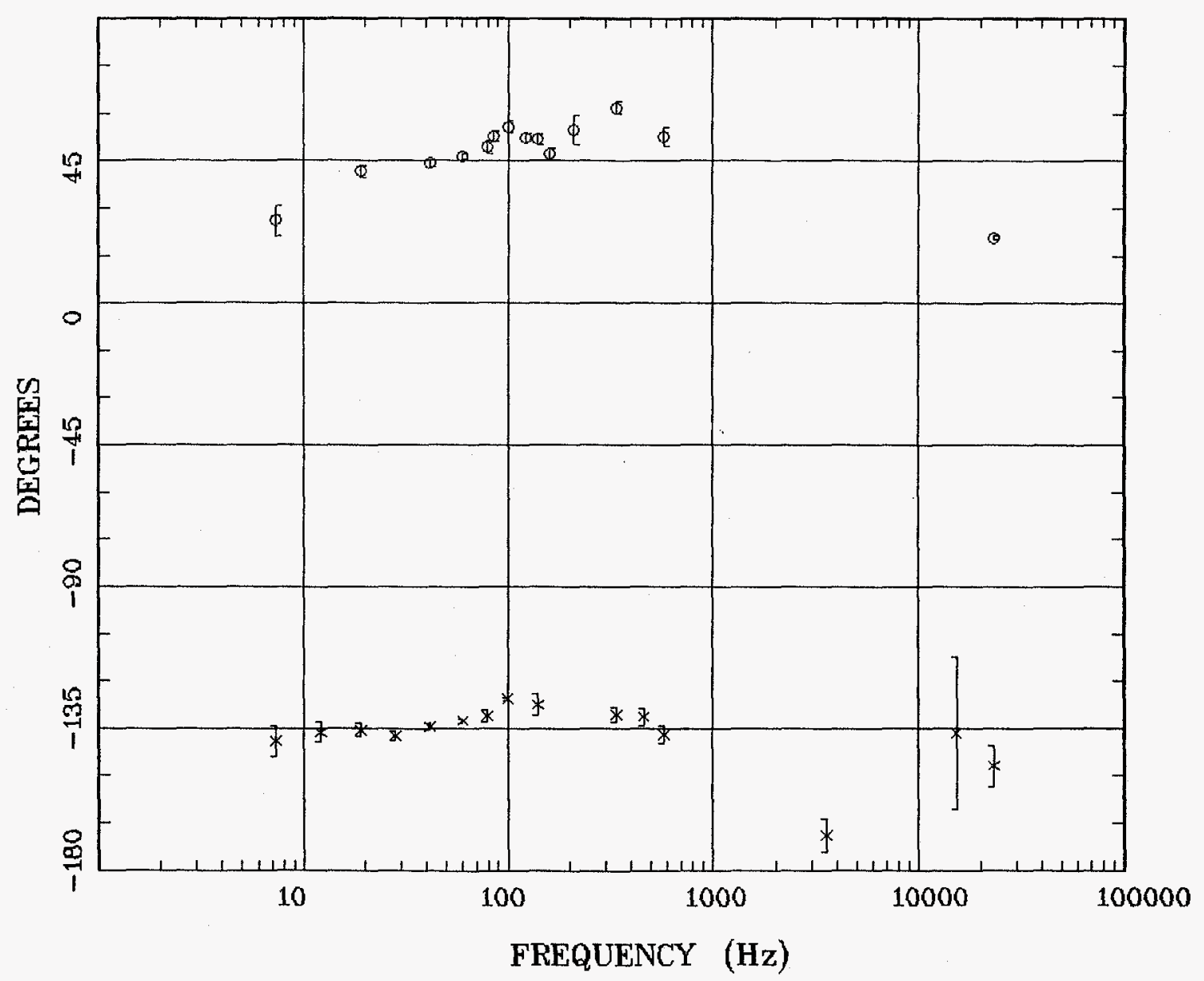

Client:

Remote: remote $\mathrm{e}$

Acquired: 14:1 Sep 05, 1997 Survey Co:USGS
Rotation:

Filename: bba10com.all

Channels: Ch1 Ch2 Ch3 Ch4 Ch5 Ch6

Plotted: 10:45 Sep 17, 1997

< EMI - ElectroMagnetic Instruments

Fig. 10c 
ROTATION ANGLE

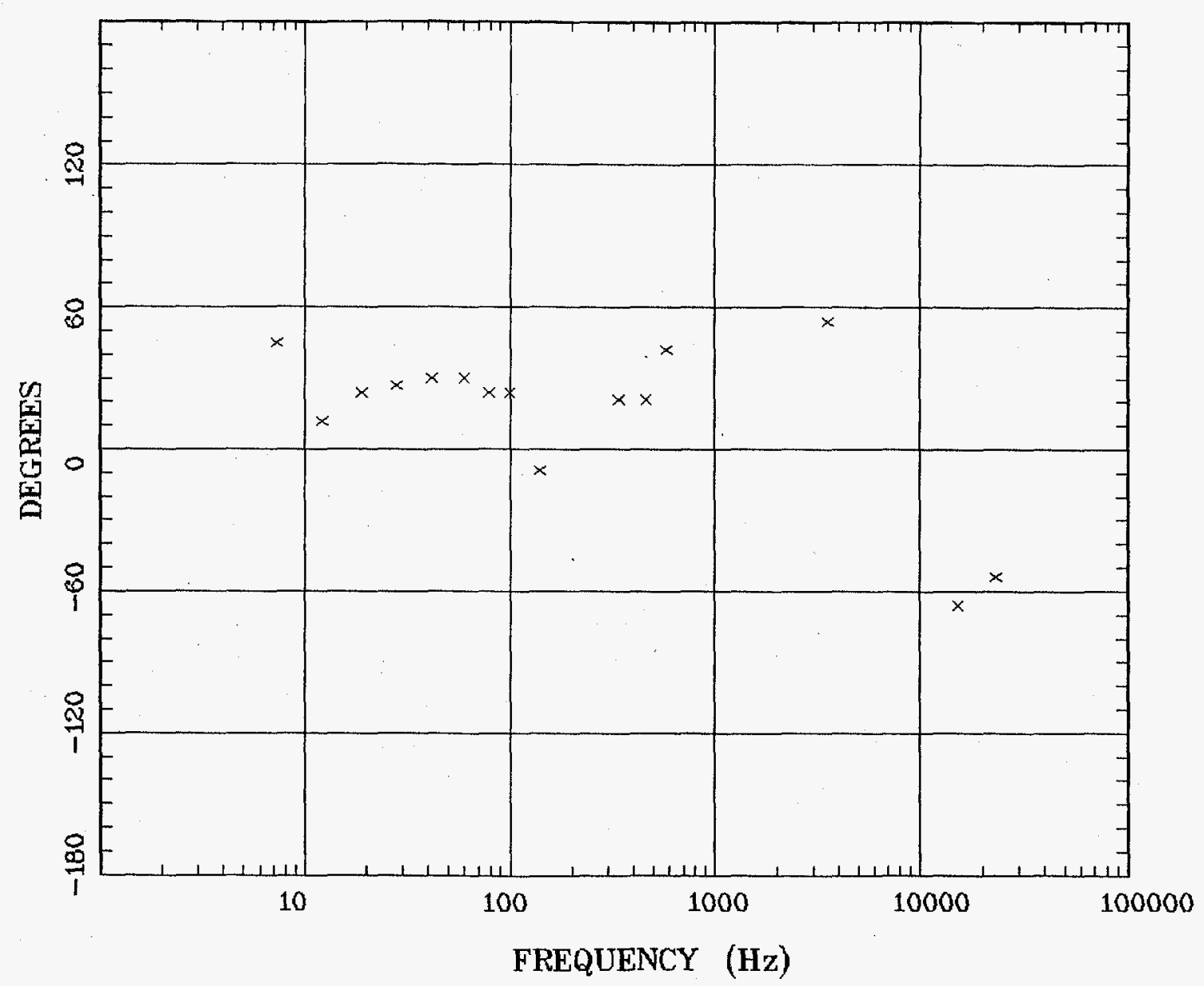

Client:

Remote: remote e

Acquired: $14: 1$ Sep 05, 1997 Survey Co:USGS
Rotation:

Filename: bba10com.all

Channels: Ch1 Ch2 Ch3 Ch4 Ch5 Ch6

Plotted: 10:45 Sep 17, 1997

< EMI - ElectroMagnetic Instruments

Fig. 10d 
IMPEDANCE SKEW

north of Beatty

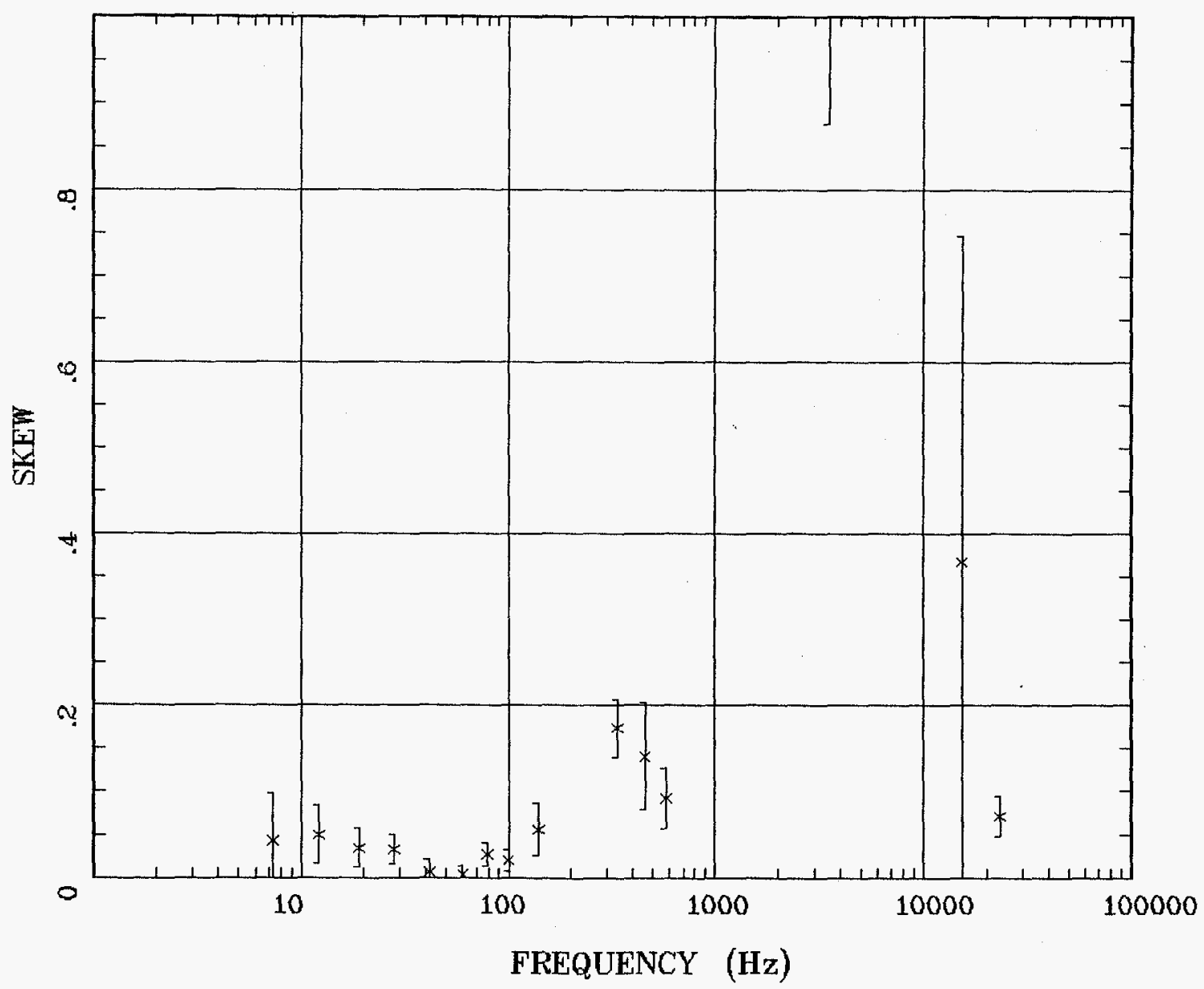

Client:

Remote: remote $\mathrm{e}$

Rotation:

Filename: bba10comall

Acquired: 14:1 Sep 05, 1997

Channels: Ch1 Ch2 Ch3 Ch4 Ch5 Ch6 Survey Co:USGS

Plotted: 10:45 Sep 17, 1997

< EMI - ElectroMagnetic Instruments

Fig. 10e 
E MULT Coh.

north of Beatty

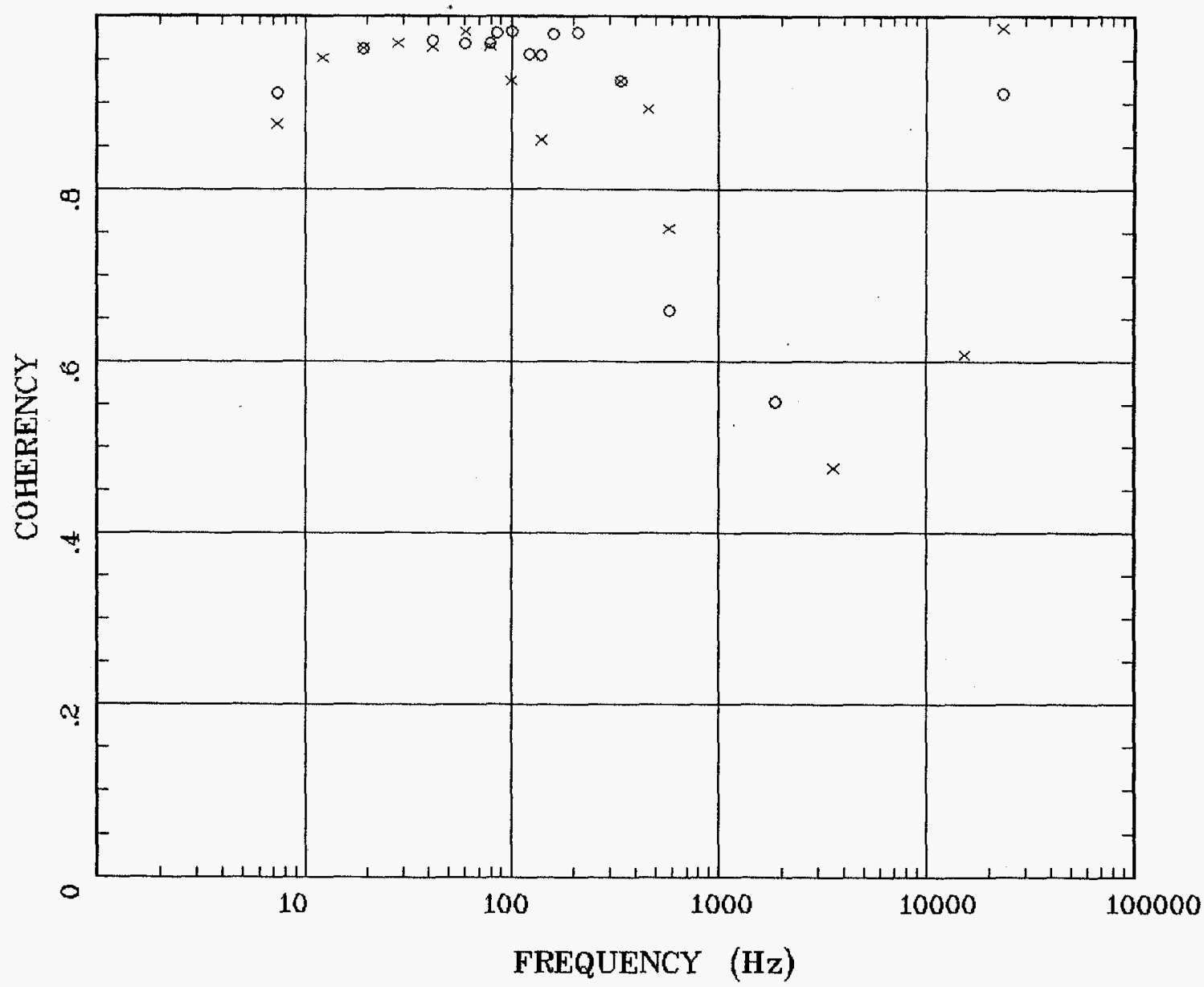

Client:

Remote: remote $\mathrm{e}$

Acquired: 14:1 Sep 05, 1997

Survey Co:USGS
Rotation:

Filename: bba10com.all

Channels: Ch1 Ch2 Ch3 Ch4 Ch5 Ch6

Plotted: 10:45 Sep 17, 1997

< EMI - ElectroMagnetic Instruments

Fig. 10f 
TIPPER MAGNITUDE

north of Beatty

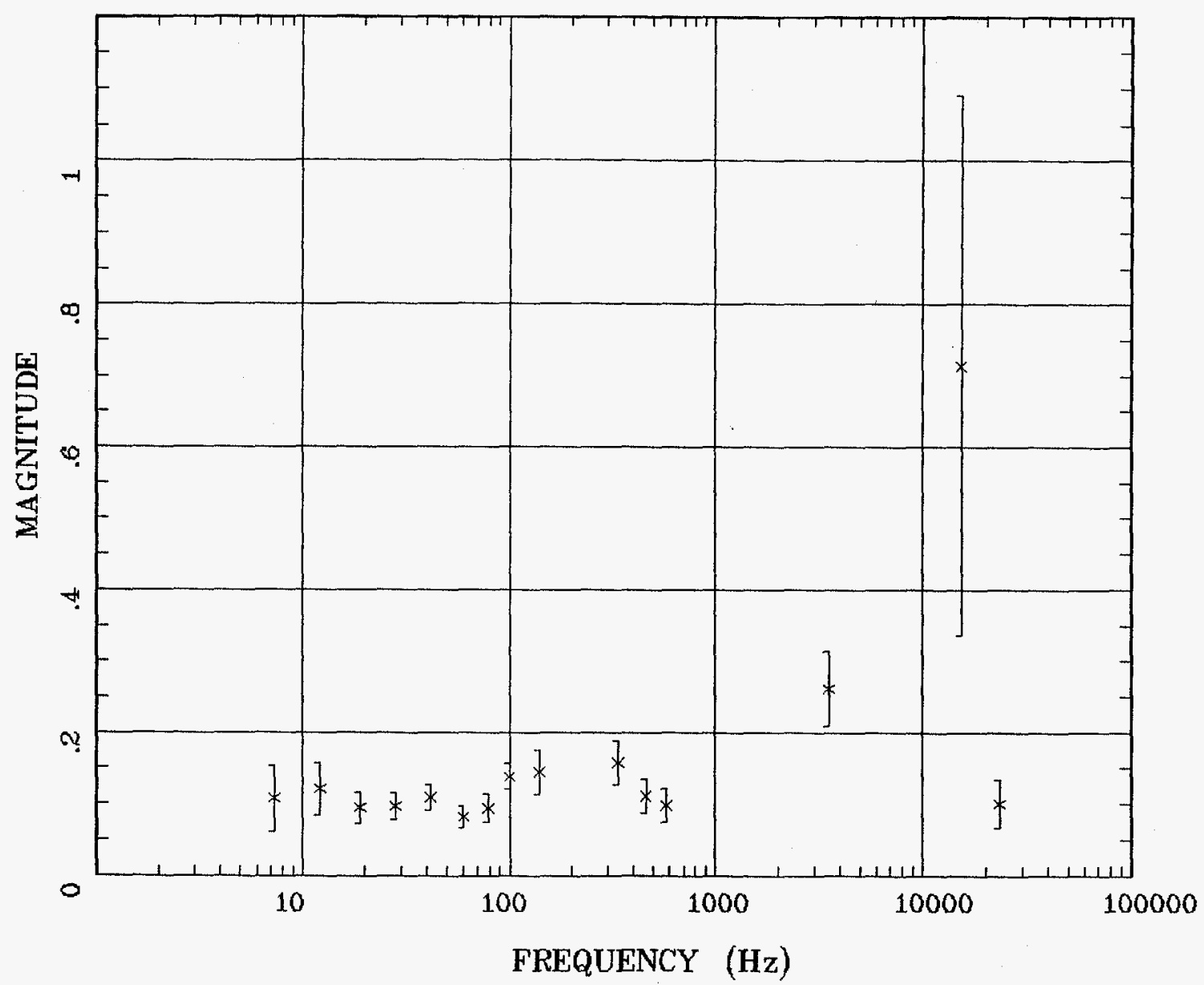

Client:

Remote: remote e

Acquired: 14:1 Sep 05, 1997 Survey Co:USGS
Rotation:

Filename: bba10com.all

Channels: Ch1 Ch2 Ch3 Ch4 Ch5 Ch6

Plotted: 10:45 Sep 17, 1997

< EMI - ElectroMagnetic Instruments

Fig. $10 \mathrm{~g}$ 
TIPPER STRIKE

north of Beatty

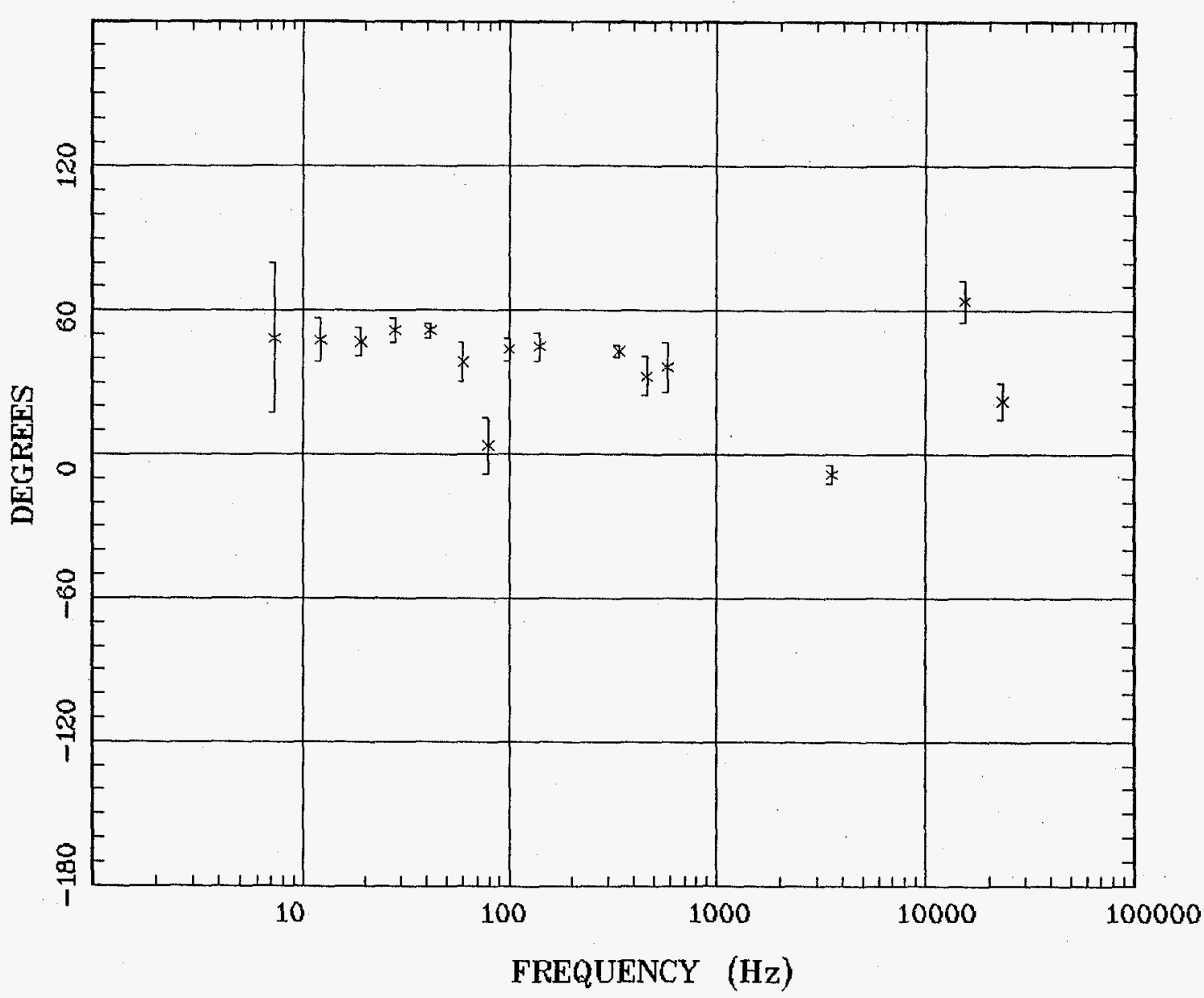

Client:

Remote: remote e

Acquired: 14:1 Sep 05, 1997 Survey Co:USGS
Rotation:

Filename: bba10com.all

Channels: Ch1 Ch2 Ch3 Ch4 Ch5 Ch6

Plotted: 10:46 Sep 17, 1997

$<$ EMI - ElectroMagnetic Instruments

Fig. 10h 


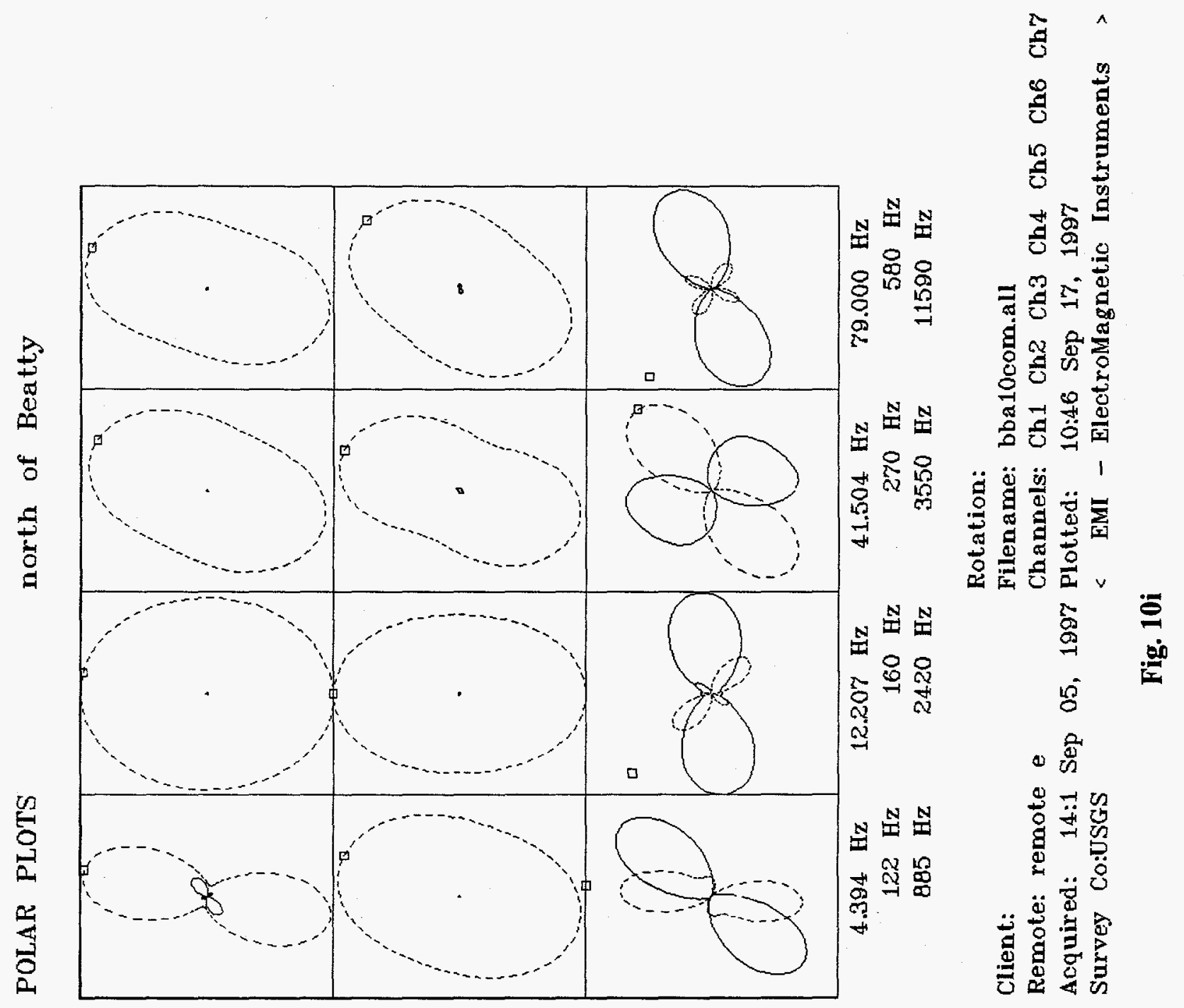


Resistivity (Bostick Inv.)

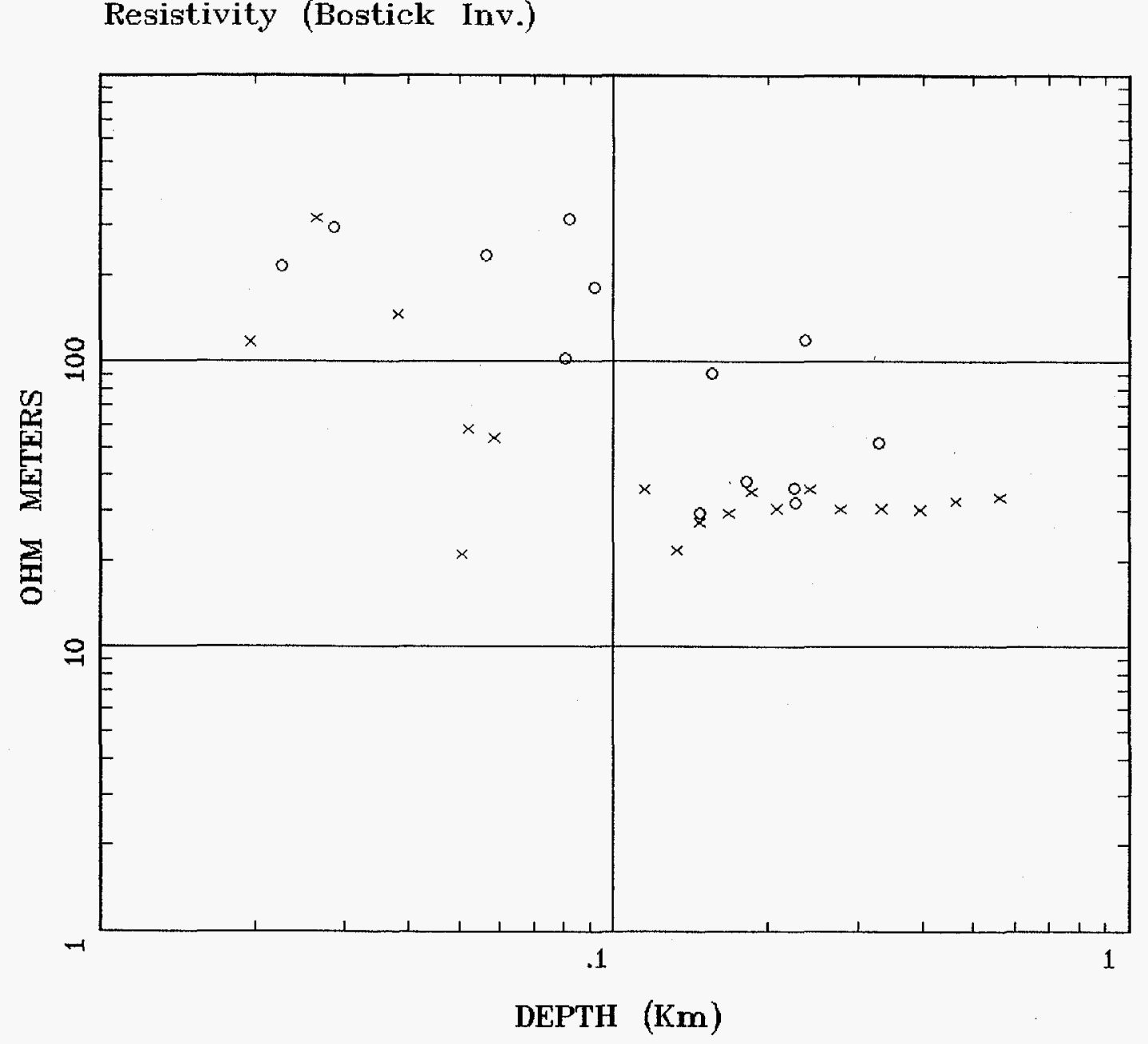

Client:

Rotation:

Remote:

Filename: bba11com.all

Channels: Ex1 Ey1 Hx1 Hy1 Hz1 Ex2

Acquired: 13:3 Sep 17, 1997

Plotted: 13:57 Sep 17, 1997

Survey Co:

$<$ EMI - ElectroMagnetic Instruments

Fig. $11 a$ 
APPARENT RESISTIVITY north of Beatty

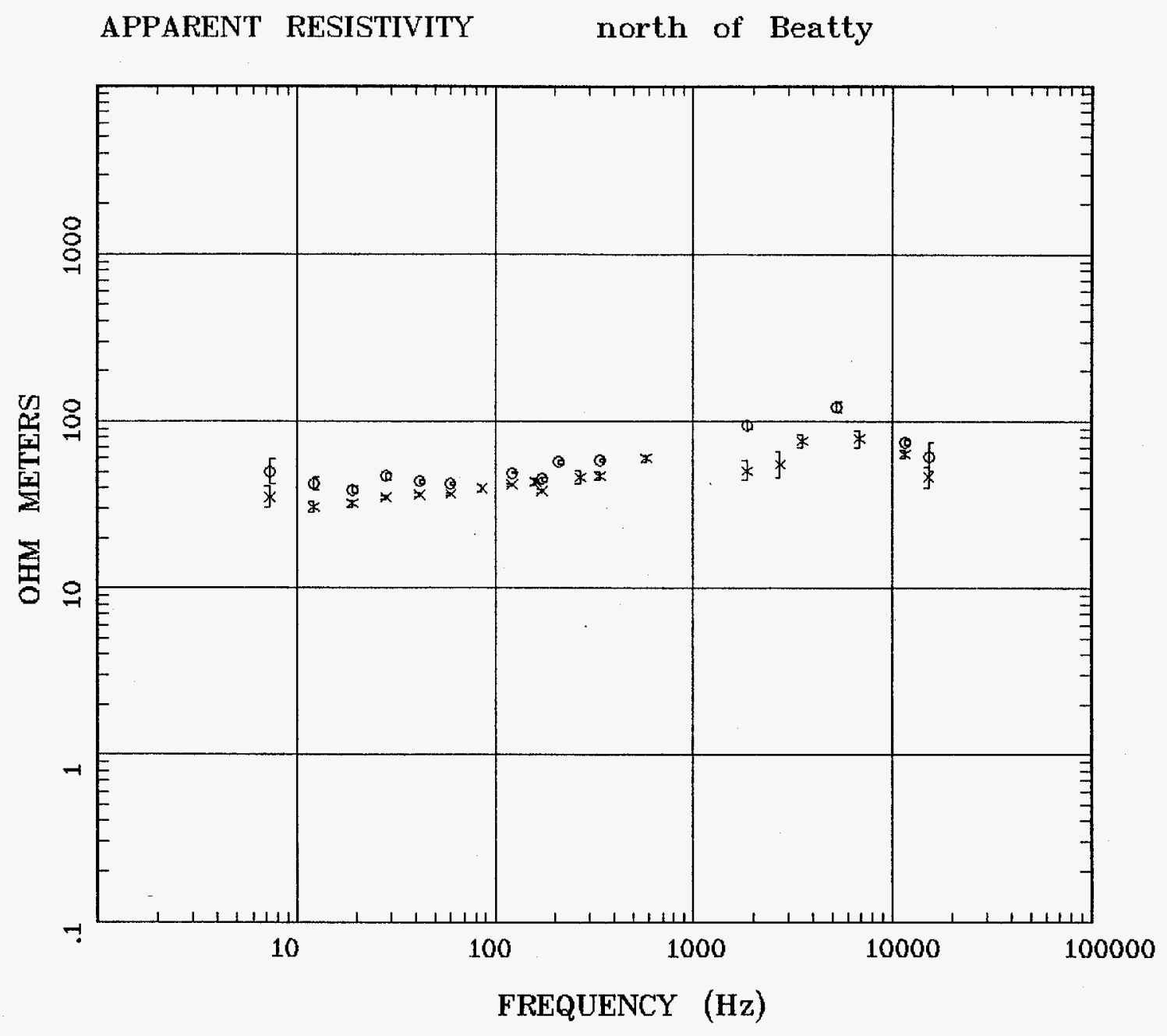

Client:

Remote: remote e

Acquired: 13:3 Sep 17, 1997

Survey Co:USGS
Rotation:

Filename: bba11com.all

Channels: Ch1 Ch2 Ch3 Ch4 Ch5 Ch6

Plotted: 13:57 Sep 17, 1997

< EMI - ElectroMagnetic Instruments

Fig. 11b 


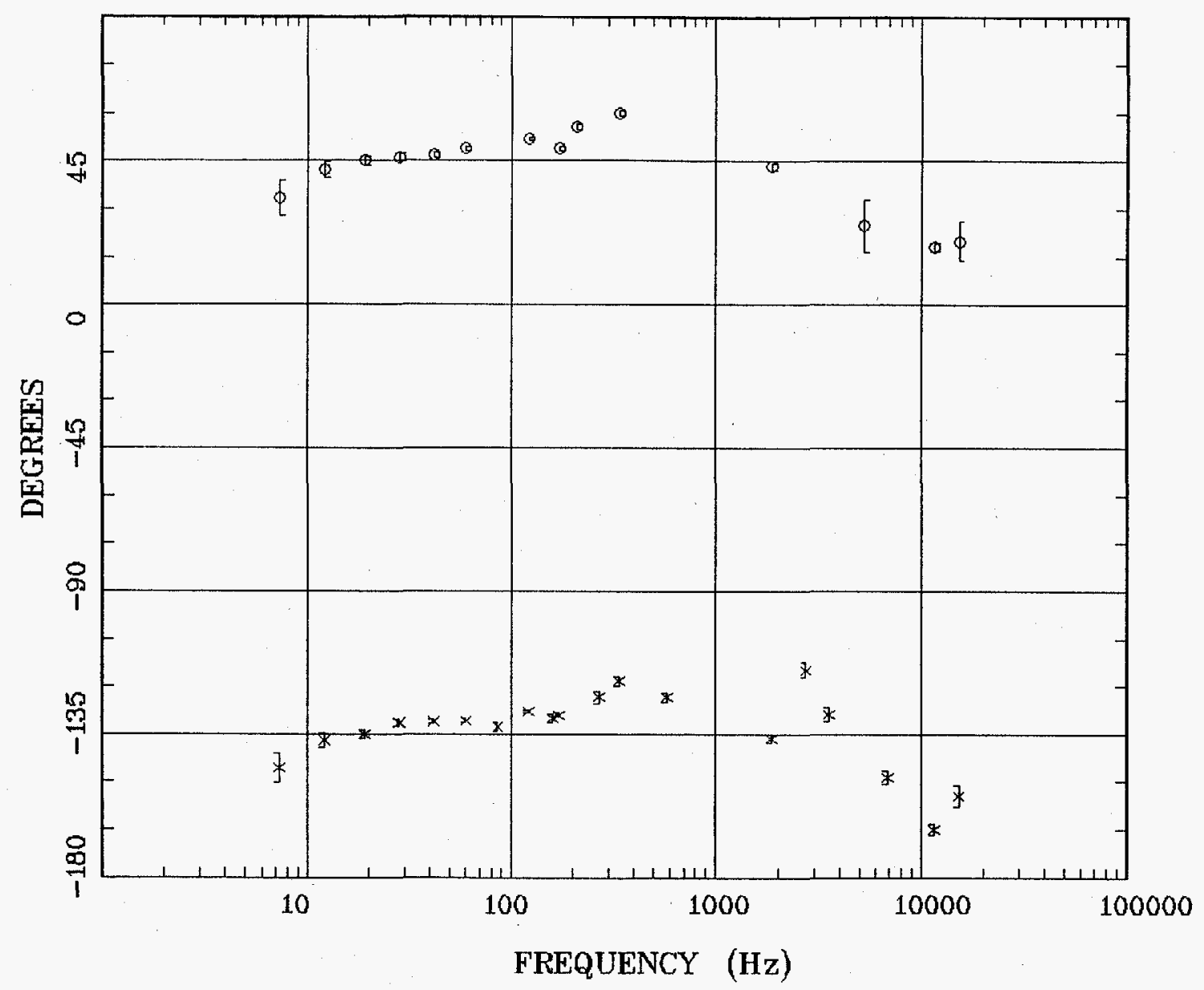

Client:

Remote: remote e Acquired: 13:3 Sep 17, 1997 Survey Co:USGS

\section{Rotation:}

Filename: bba11com.all

Channels: Ch1 Ch2 Ch3 Ch4 Ch5 Ch6

Plotted: 13:57 Sep 17, 1997

$<$ EMI - ElectroMagnetic Instruments

Fig. 11c 


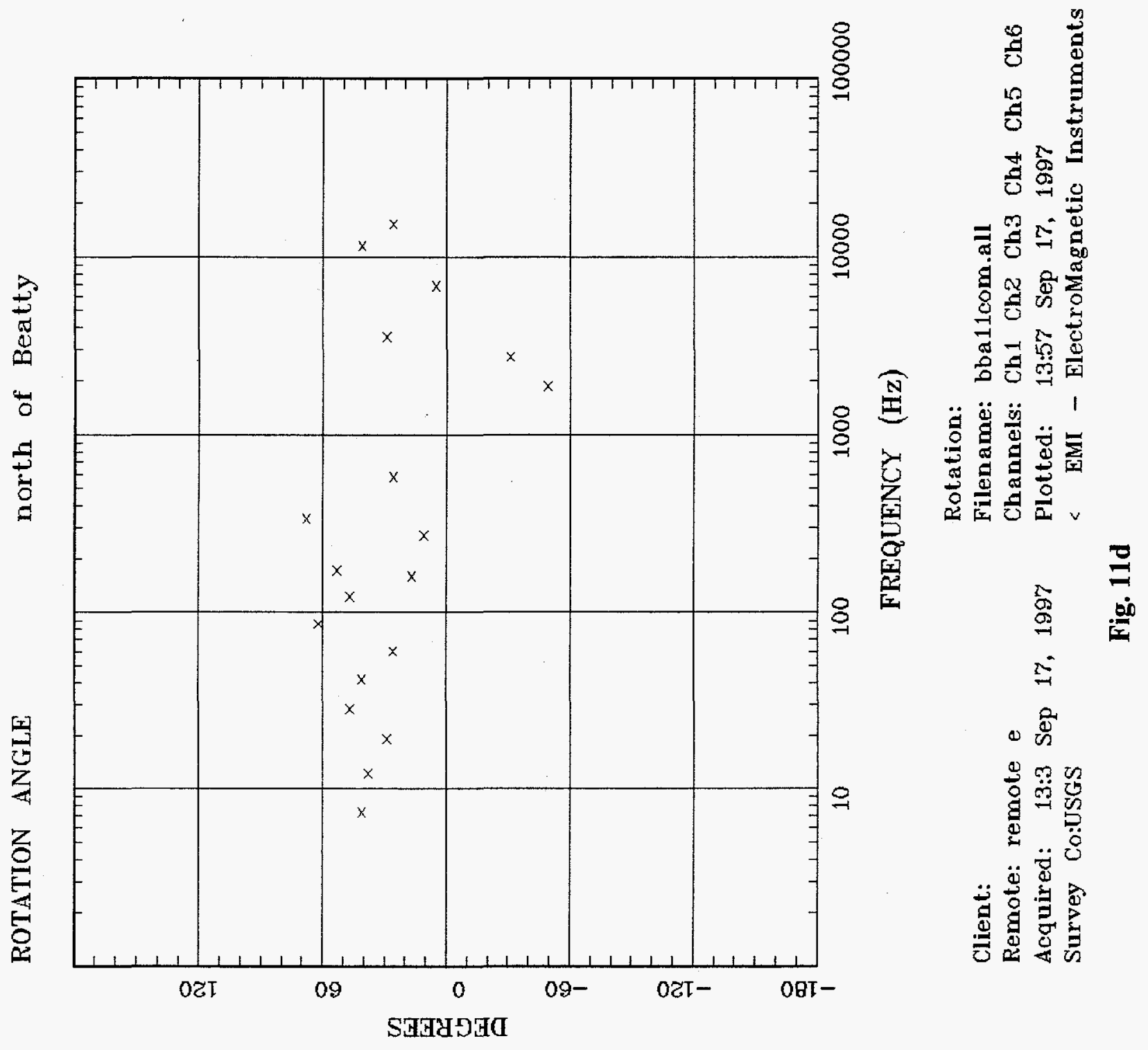


IMPEDANCE SKEW

north of Beatty

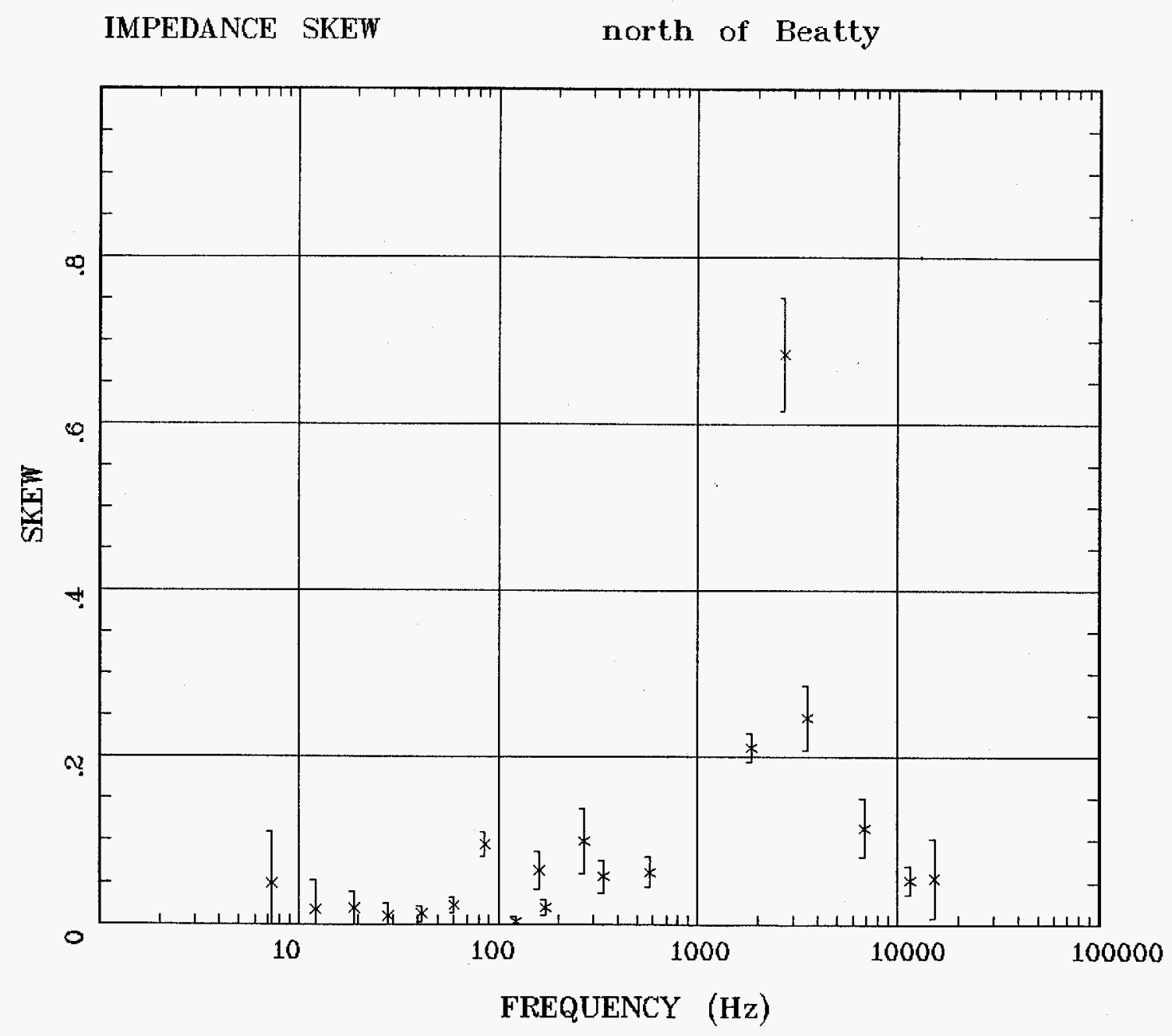

Client:

Rotation:

Remote: remote $\mathrm{e}$

Filename: bba11com.all

Acquired: 13:3 Sep 17, 1997

Channels: Ch1 Ch2 Ch3 Ch4 Ch5 Ch6

Survey Co:USGS

Plotted: 13:57 Sep 17, 1997

< EMI - ElectroMagnetic Instruments

Fig. 11e 


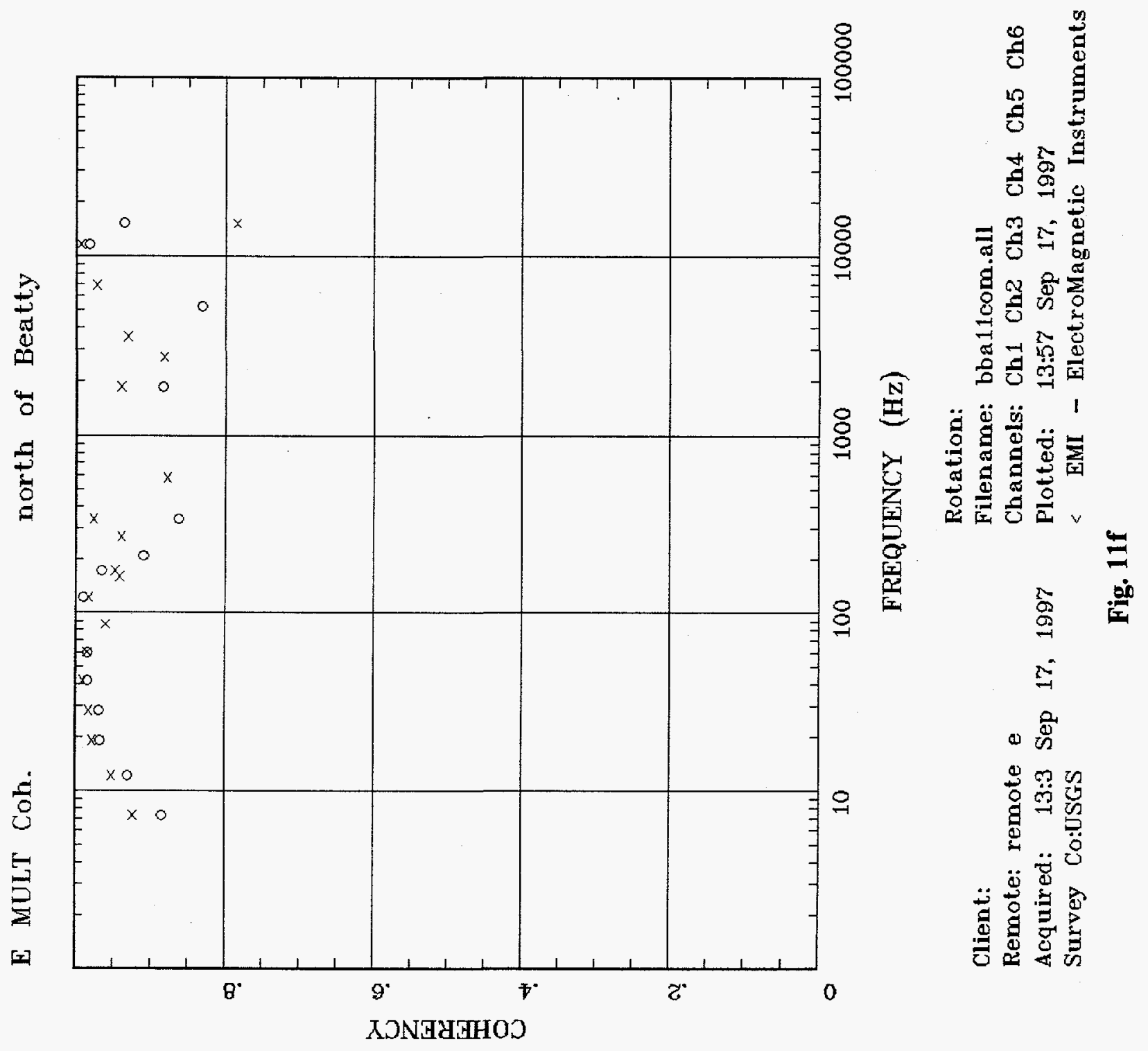




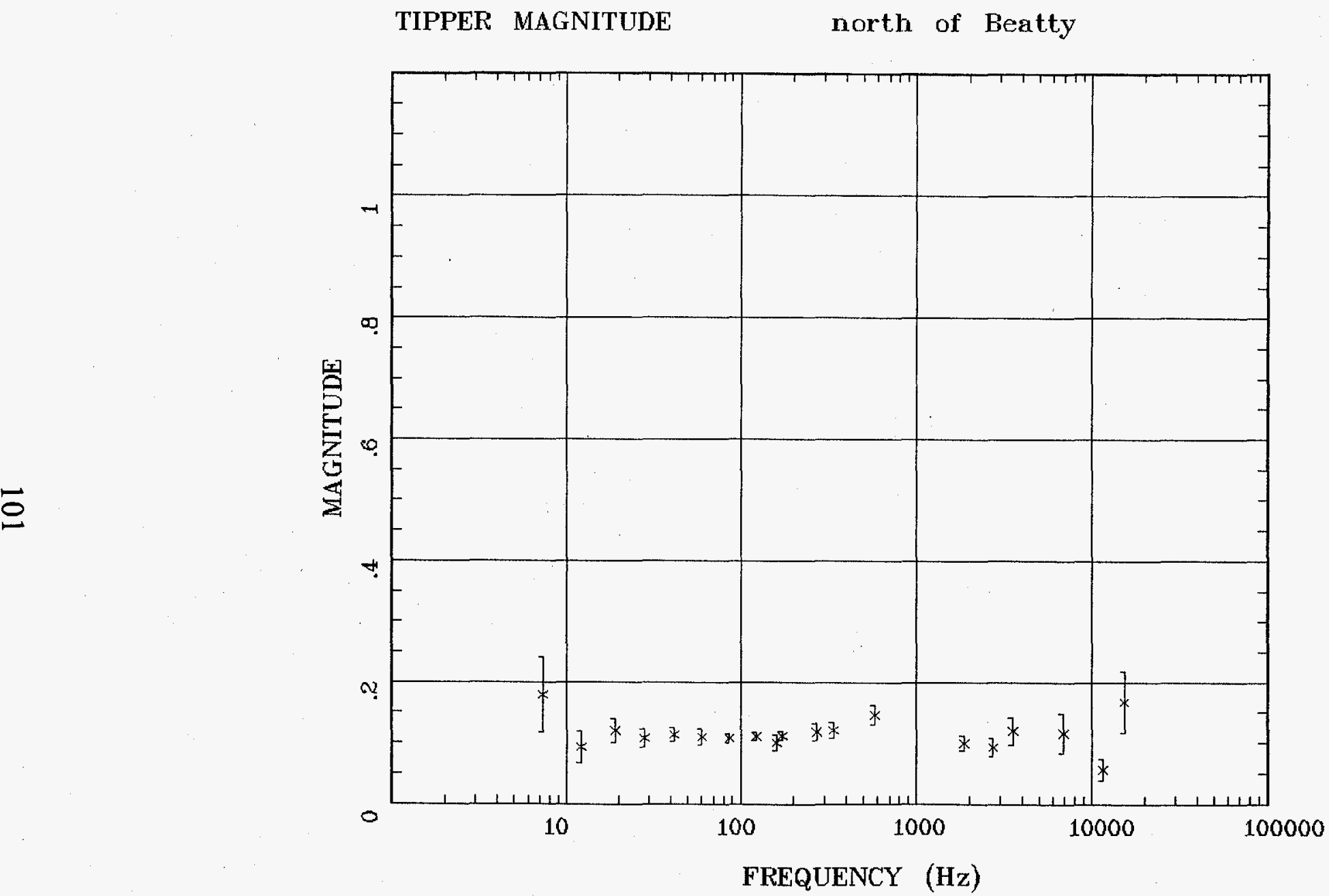

Client:

Rotation:

Remote: remote $\mathrm{e}$

Acquired: $13: 3$ Sep 17; 1997

Filename: bba11com.all

Channels: Ch1 Ch2 Ch3 Ch4 Ch5 Ch6

Plotted: 13.58 Sep 17, 1997

Survey Co:USGS

< EMI - ElectroMagnetic Instruments

Fig. 11g 


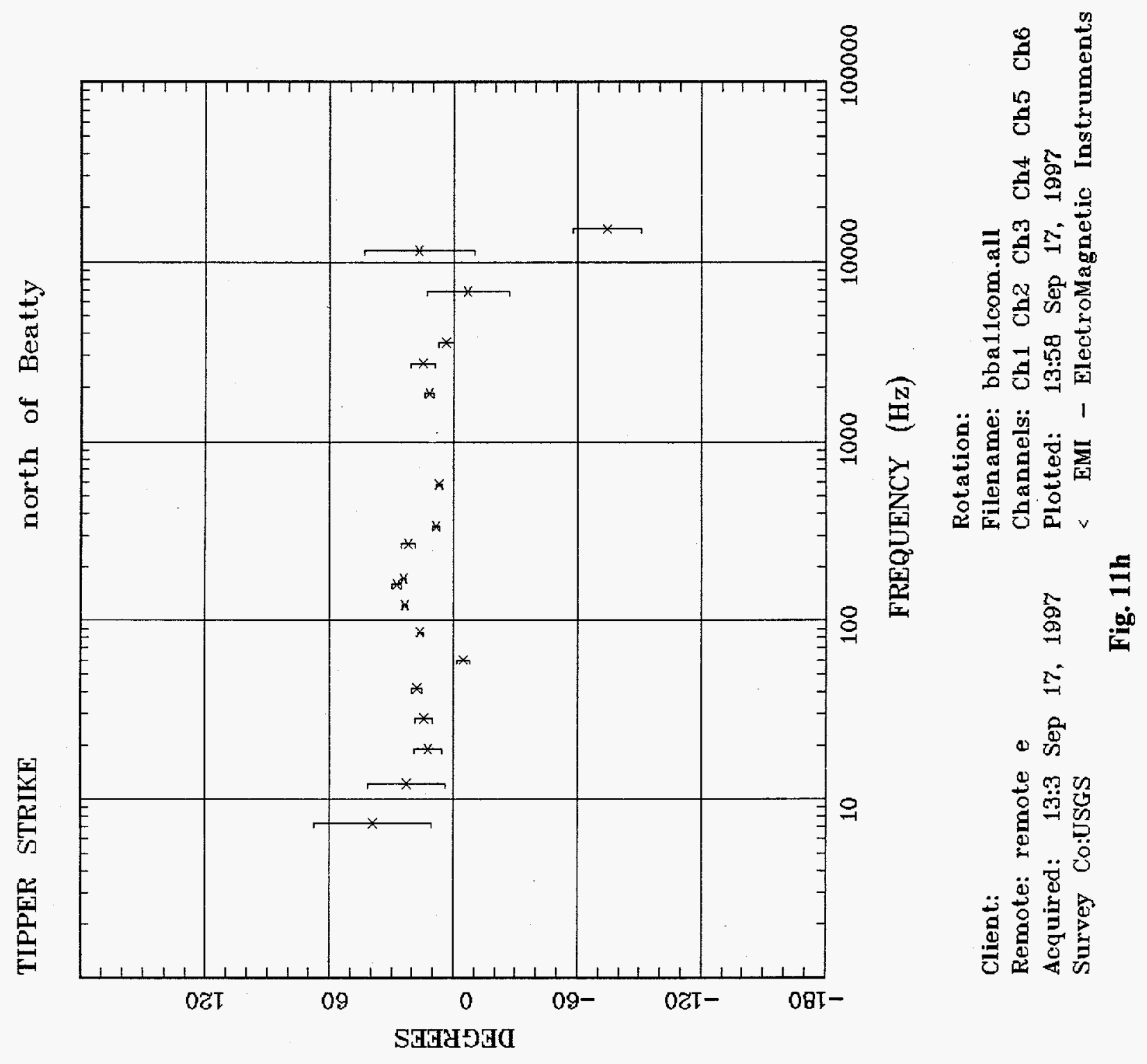




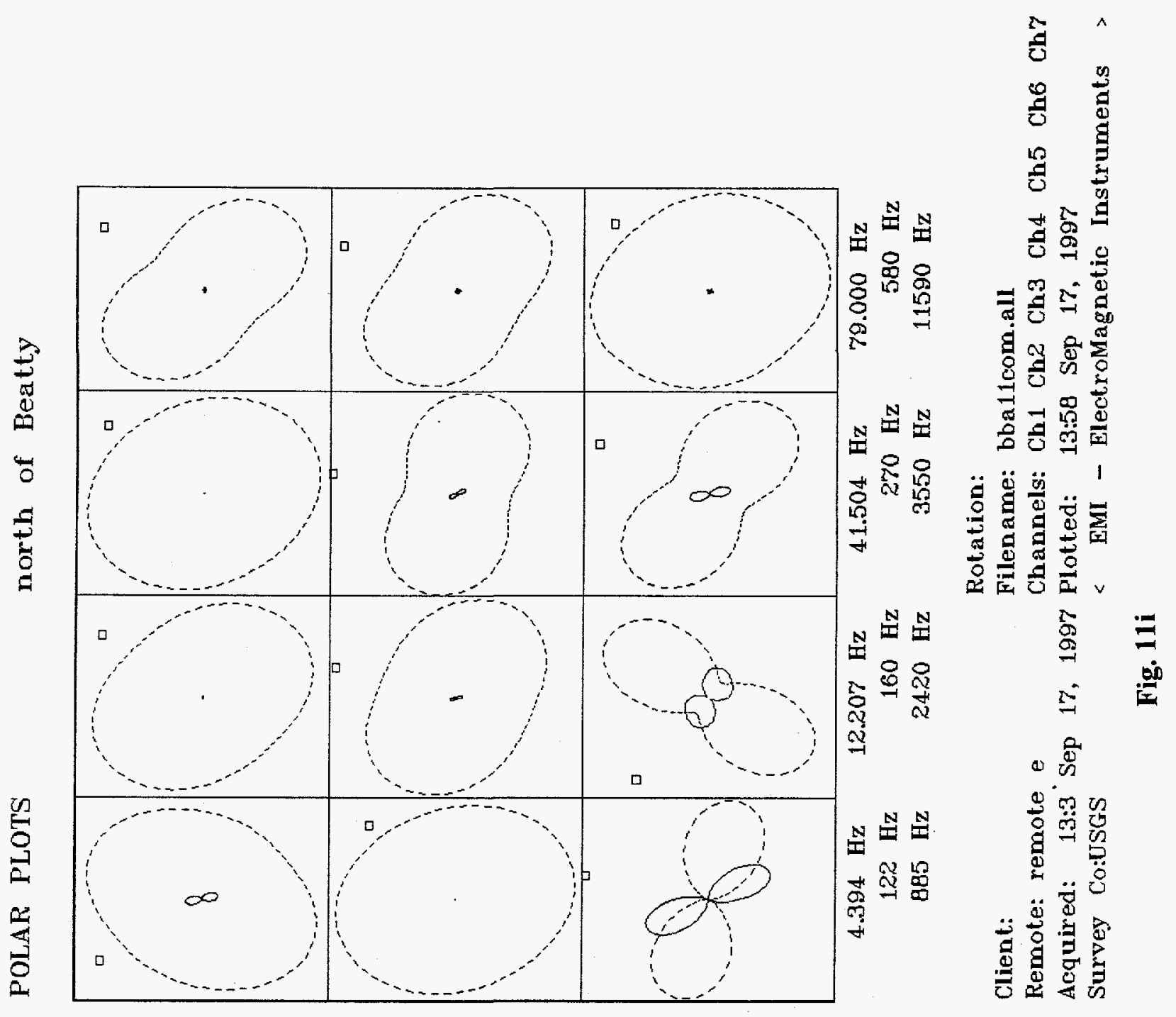




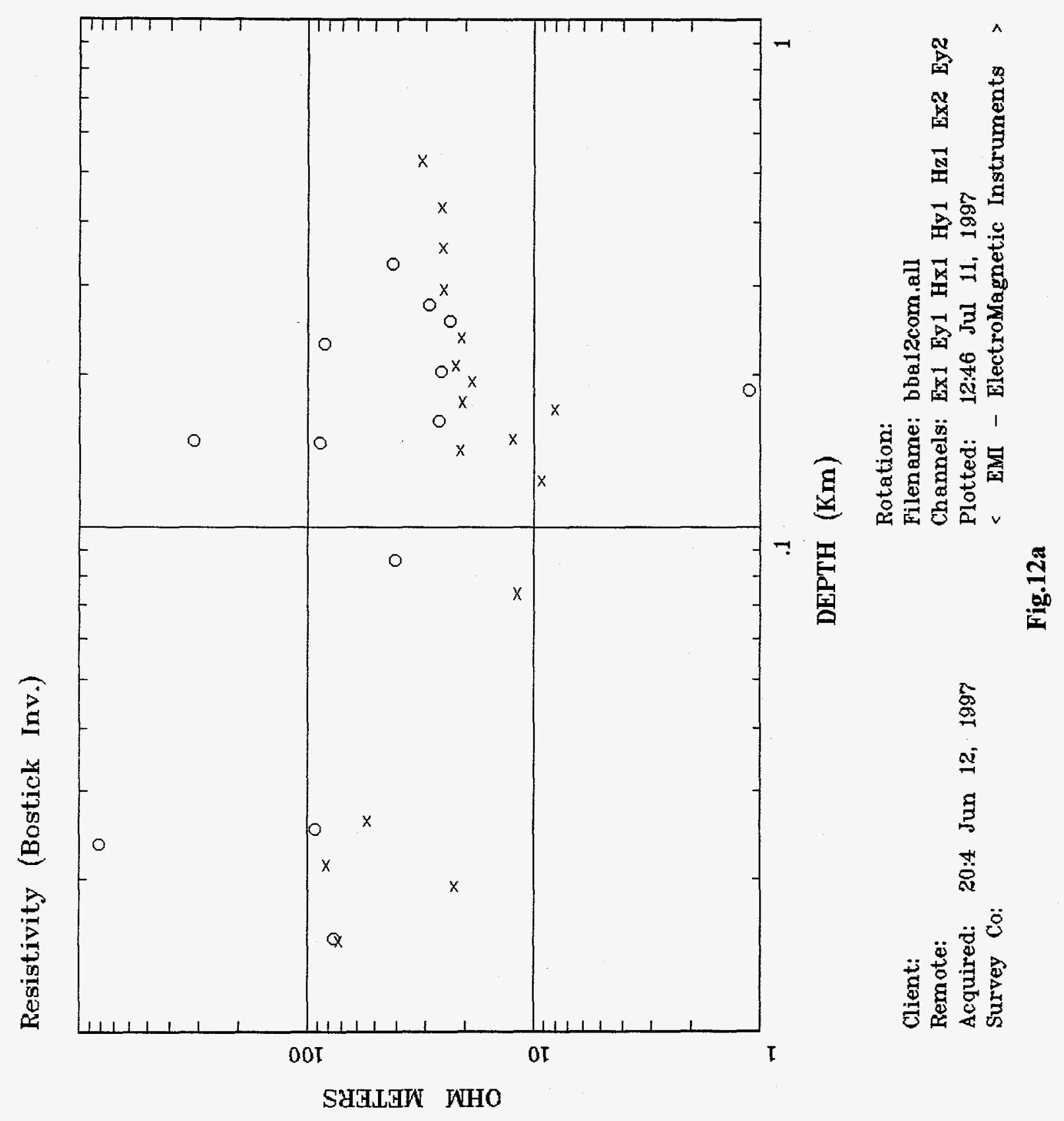




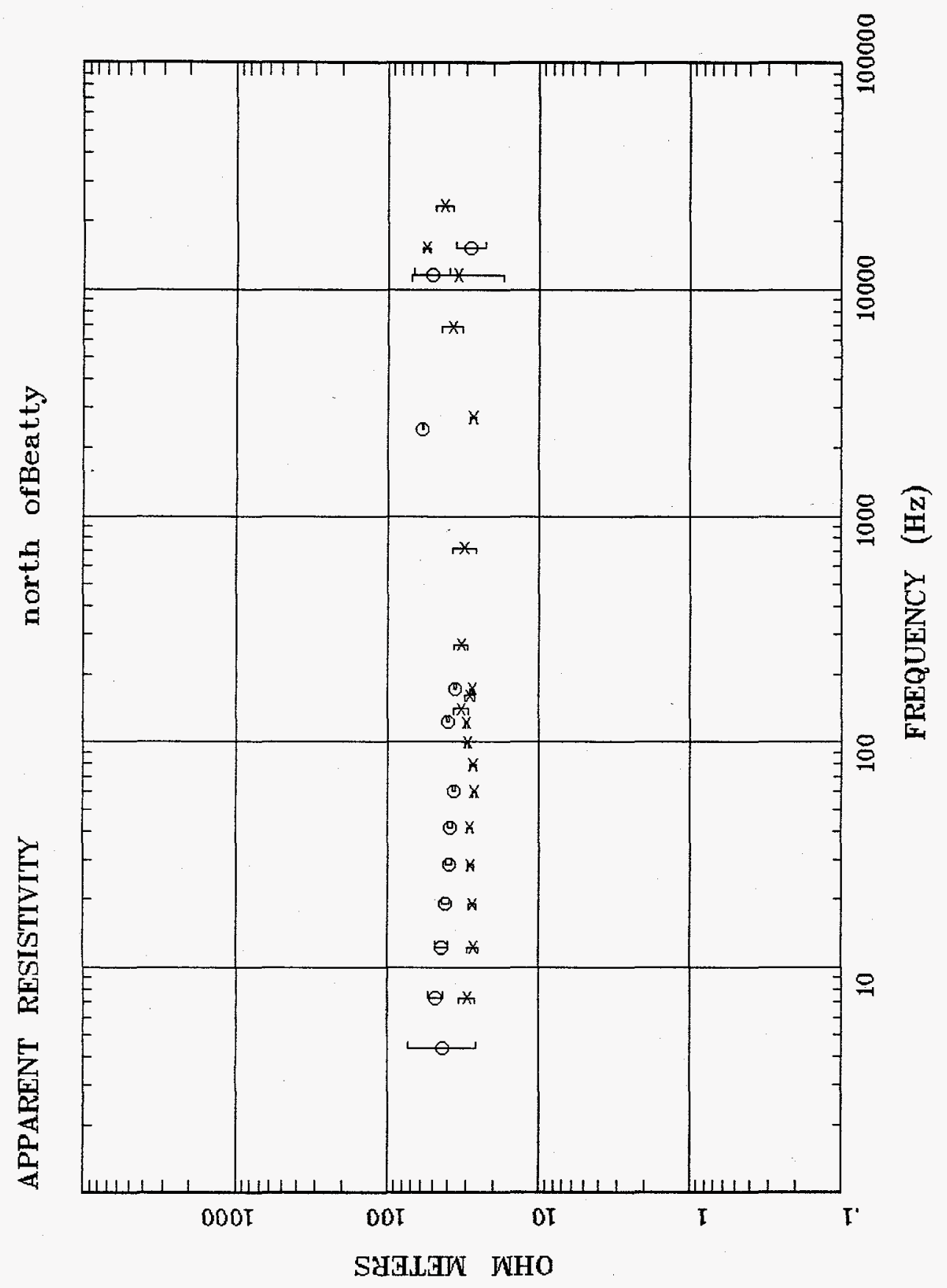

है

\&

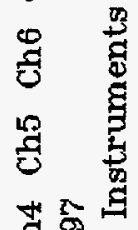

द्व

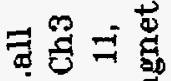

ํㅕㅇ 콜

电促

骂焉必

N $\because \ddot{1} \quad 1$

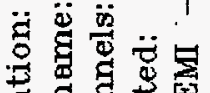

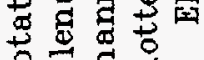

跬式的、

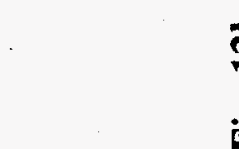

슴

疍

a

๑

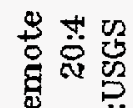

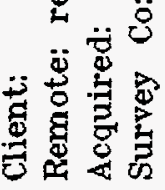

i

舫

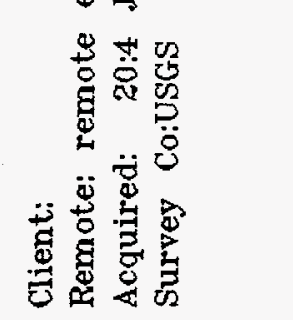




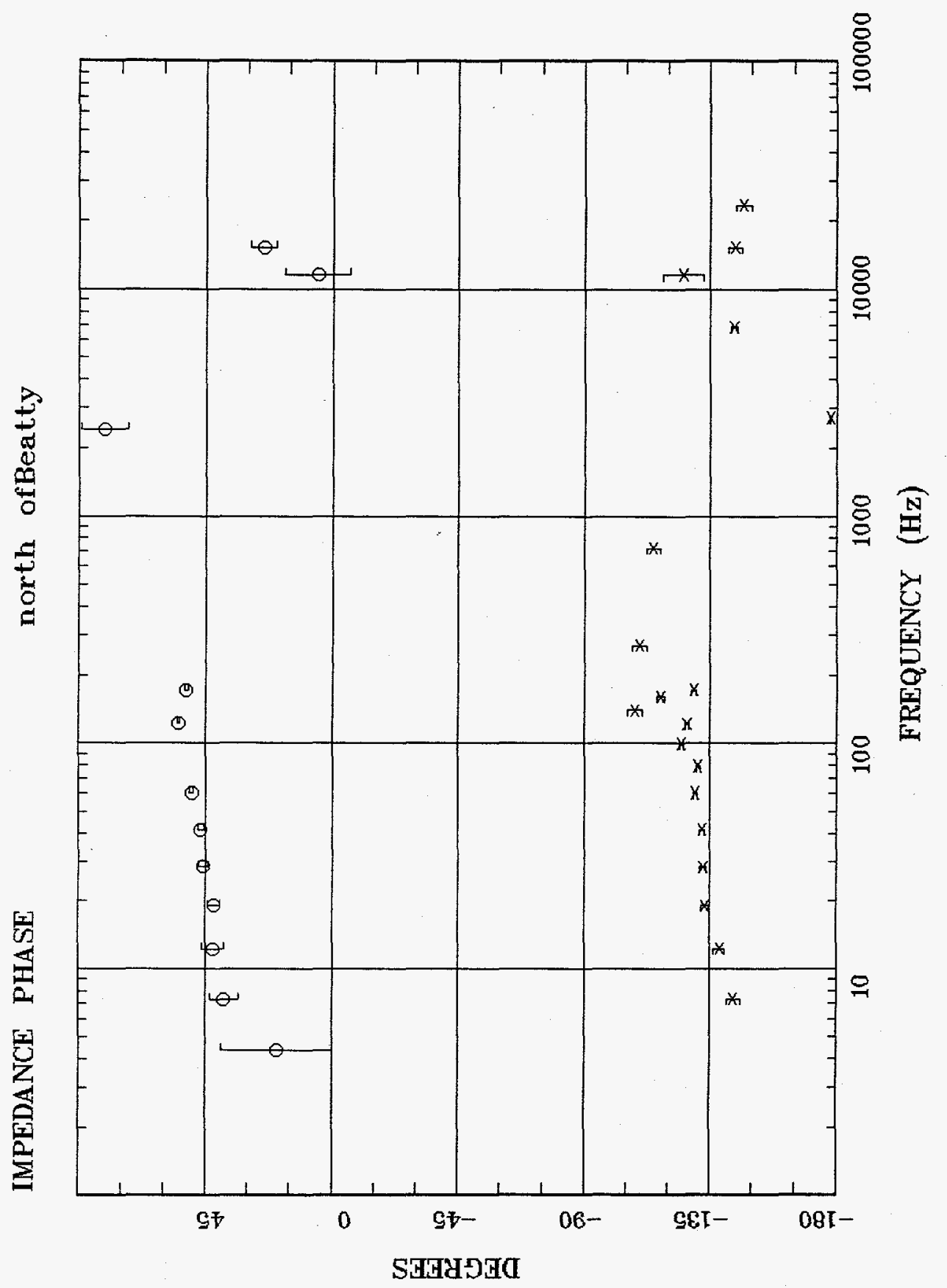

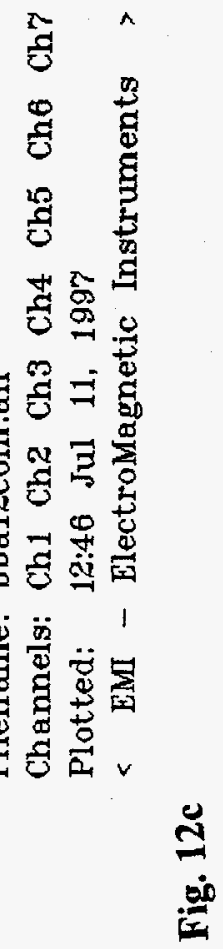

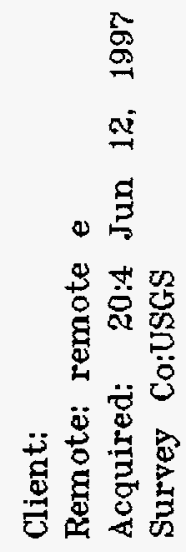



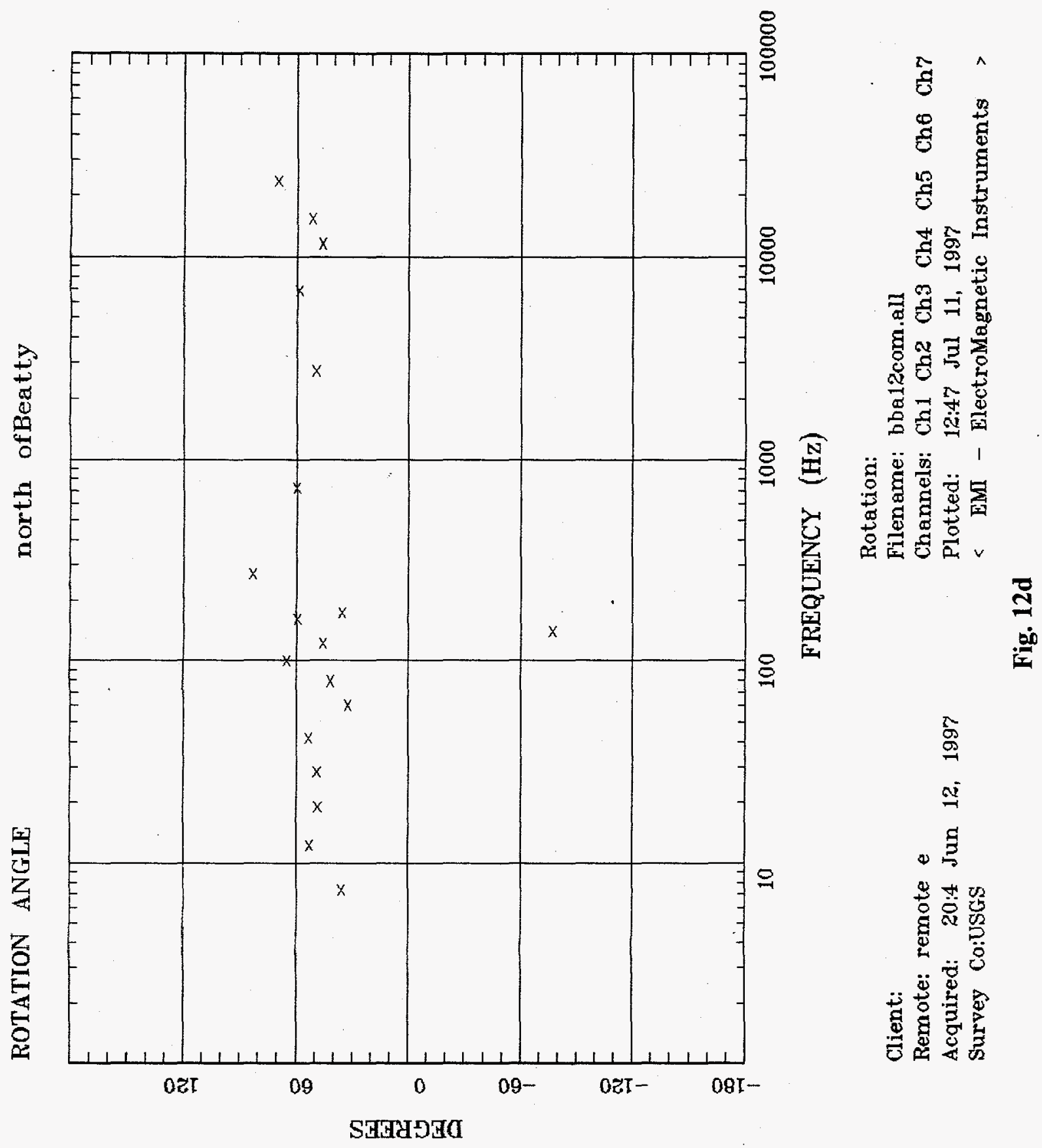


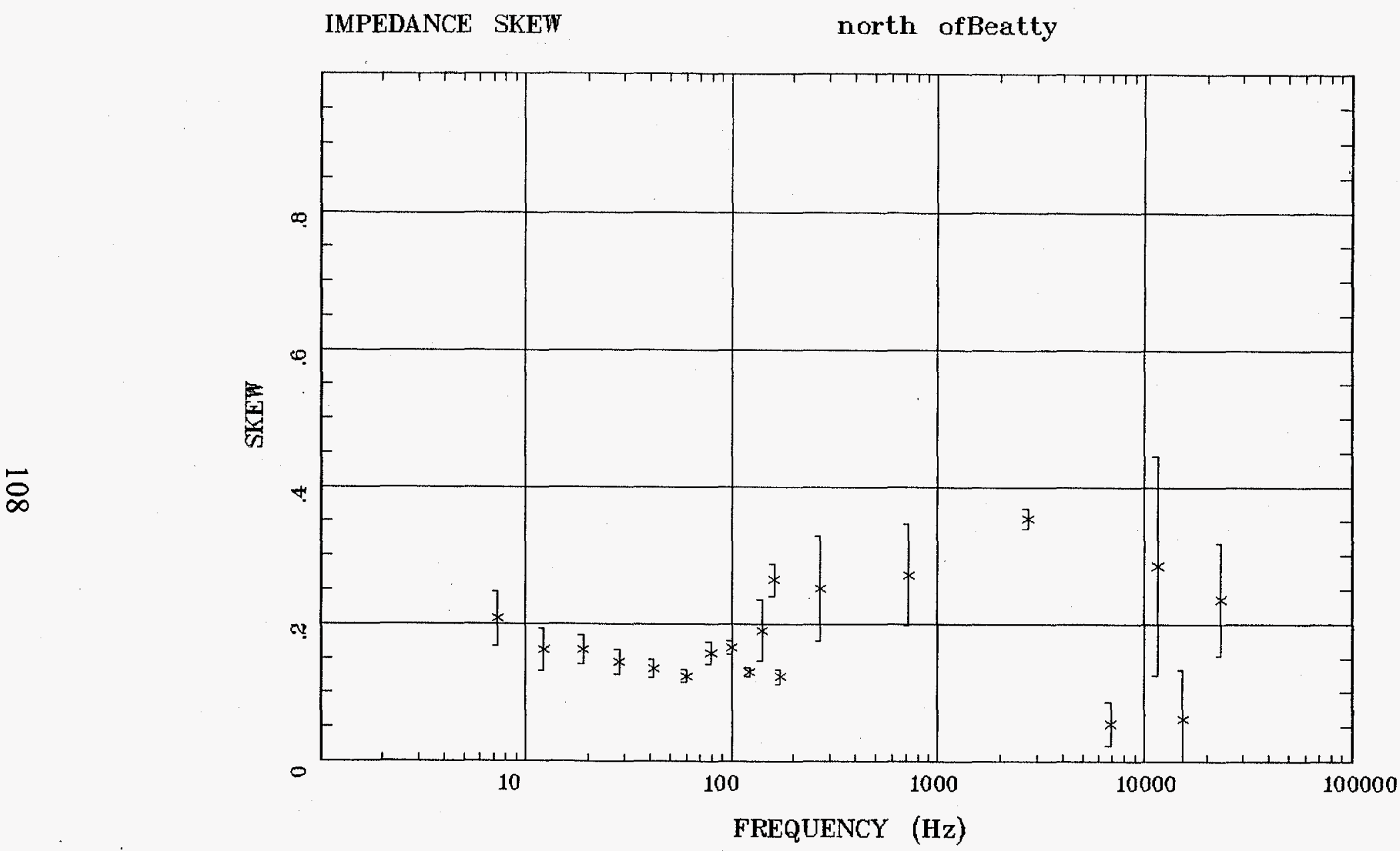

Client:

Remote: remote e

Acquired: 20:4 Jun 12, 1997 Survey Co:USGS
Rotation:

Filename: bba12com.all

Channels: Ch1 Ch2 Ch3 Ch4 Ch5 Ch6 Ch7

Platted: 12:47 Jul 11, 1997

< EMI - ElectroMagnetic Instruments >

Fig. 12e 
E MULT Coh.

north ofBeatty

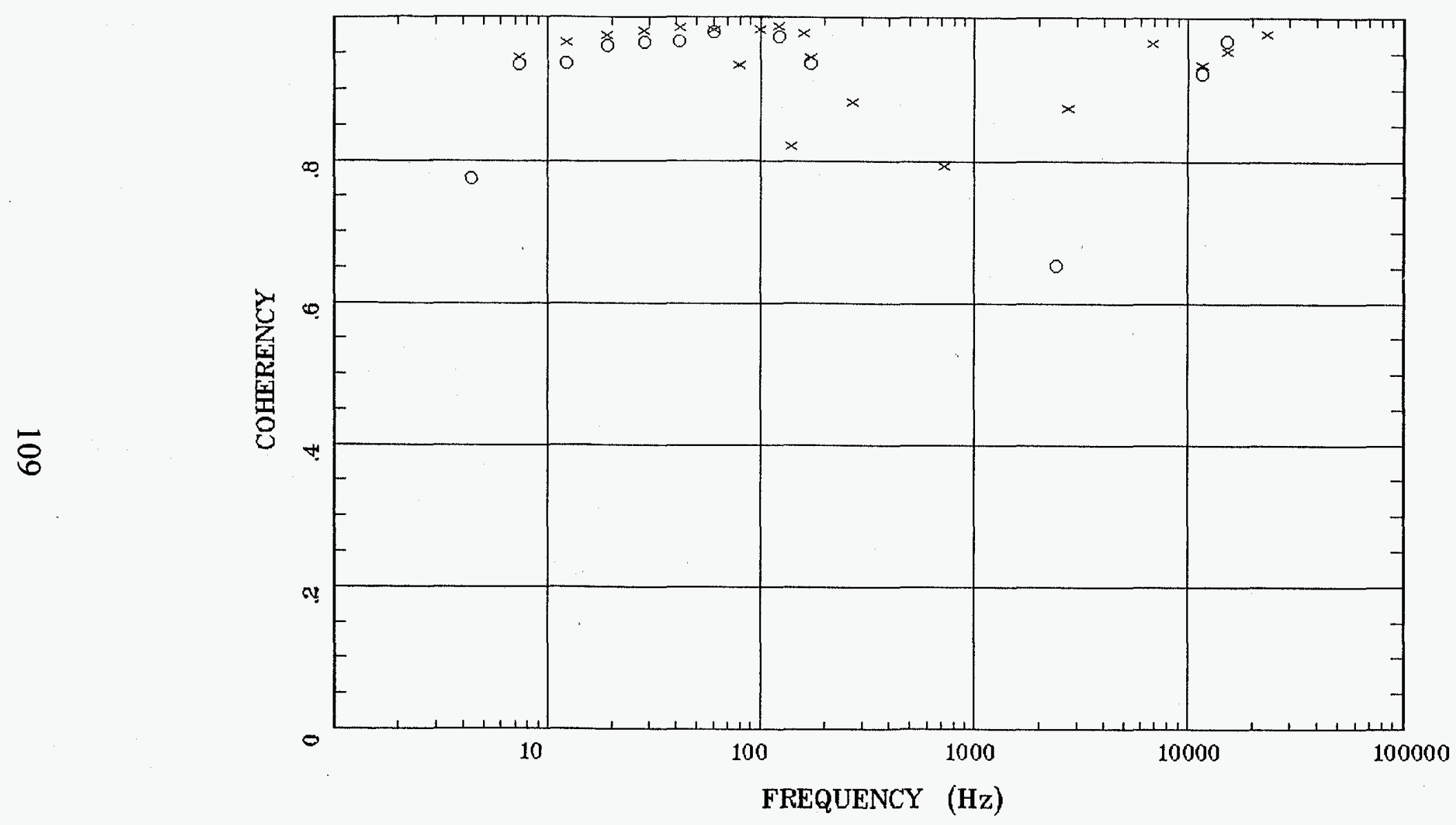

Client:

Remote: remote $e$

Acquired: 20:4 Jun 12, 1997 Survey Co:USGS
Rotation:

Filename: bba12com.all

Channels: Ch1 Ch2 Ch3 Ch4 Ch5 Ch6 Ch7

Plotted: 12:47 Jul 11, 1997

< EMI - ElectroMagnetic Instruments >

Fig. $12 f$ 


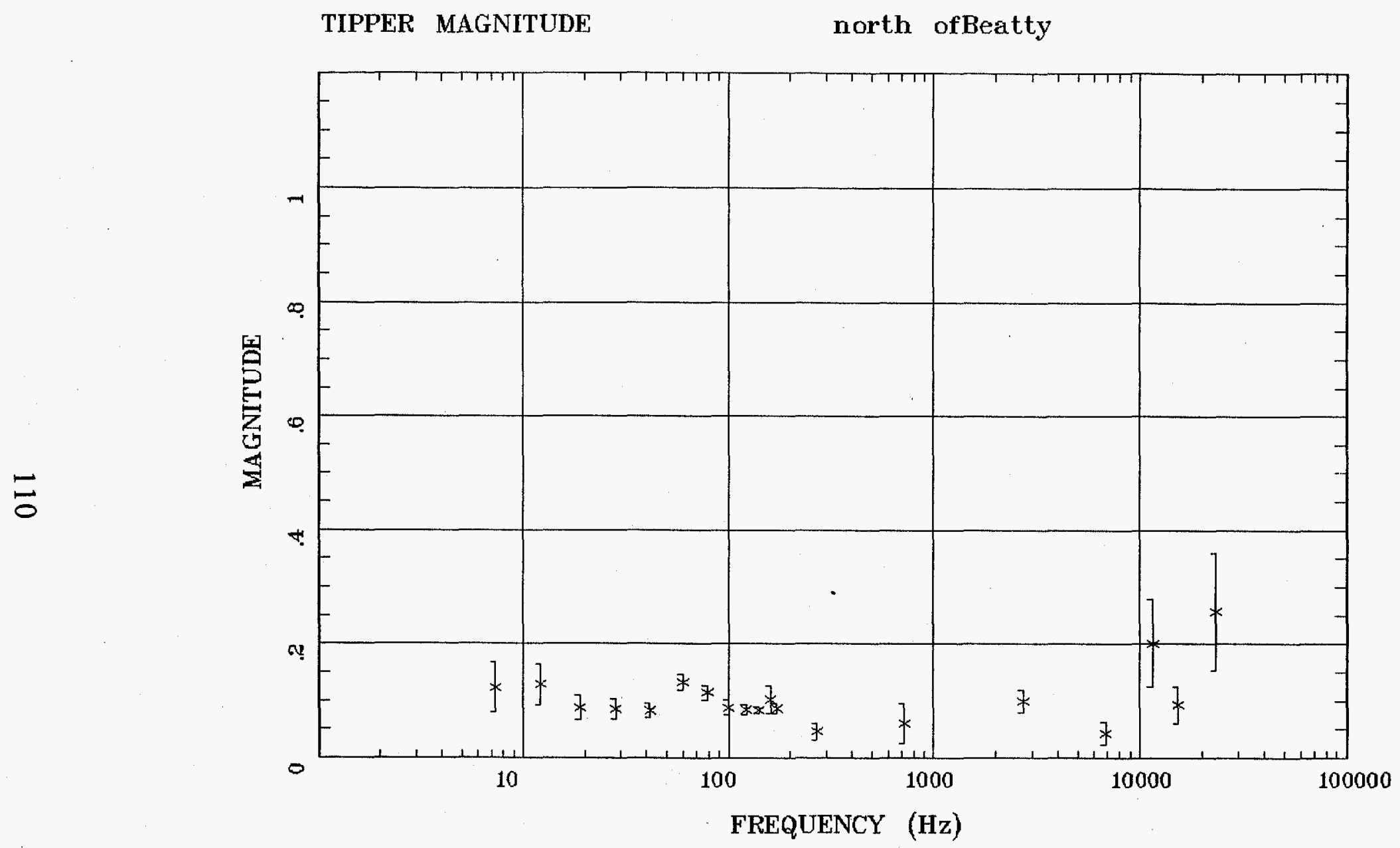

Client:

Rotation:

Filename: b̌ba1zcom.all

Remote: remote $\mathrm{e}$

Channels: Ch1 Ch2 Ch3 Ch4 Ch5 Ch6 Ch7

Acquired: 20:4 Jun 12, 1997

Plotted: 12:47 Jul 11, 1997

Survey Co:USGS

< EMI - ElectroMagnetic Instruments >

Fig. 12g 


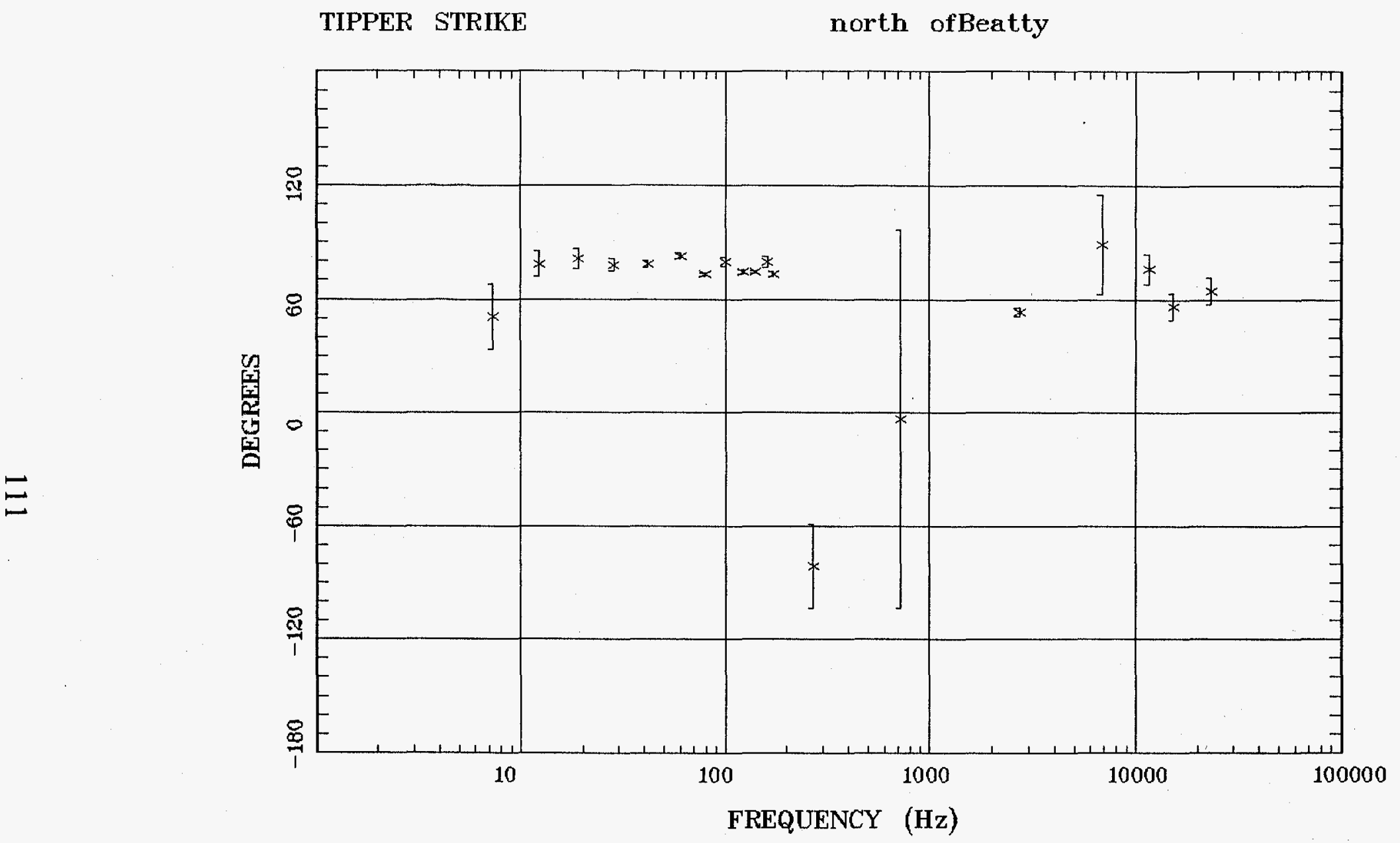

Client:

Remote: remote e

Acquired: 20:4 Jun 12, 1997 Survey Co:USGS
Rotation:

Filename: bba12com.all

Channels: Ch1 Ch2 Ch3 Ch4 Ch5 Ch6 Ch7

Plotted: 12:47 Jul 11, 1997

< EMI - ElectroMagnetic Instruments >

Fig. 12h 
north ofBeatty

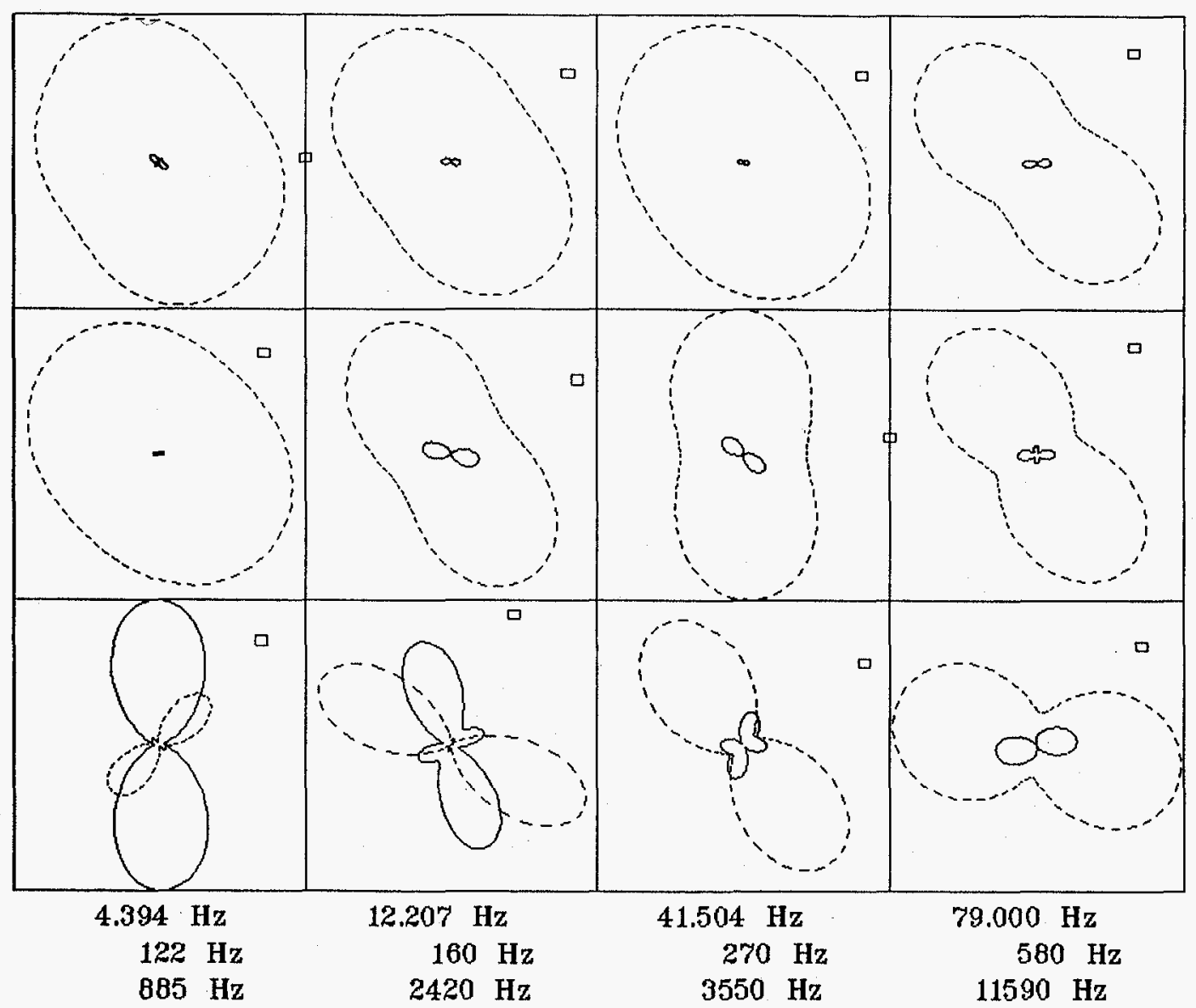

Client:

Rotation:

Remote: remote $\mathrm{e}$

Filename: bba12com.all

Acquired: 20:4 Jun 12, 1997

Channels: Ch1 Ch2 Ch3 Ch4 Ch5 Ch6 Chy Survey Co:USGS

Platted: 12:47 Jul 11, 1997

< EMI - ElectroMagnetic Instruments >

Fig. 12i 


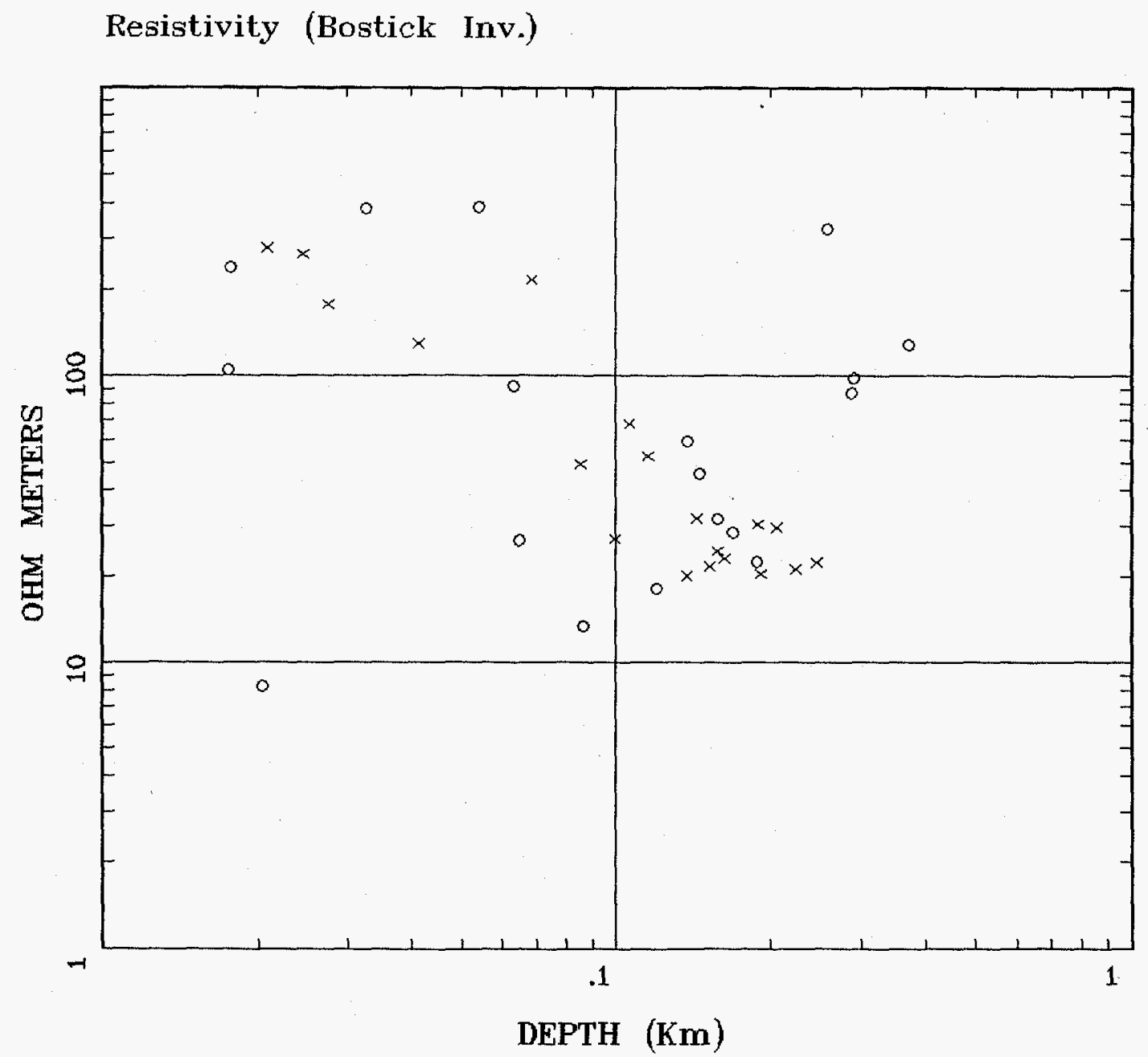

Client:

Remote:

Acquired: 20:0 Jun 12, 1997 Survey Co:
Rotation:

Filename: bba13comb.all

Channels: Ex1 Ey1 Hx1 Hy1 Hz1 Ex2

Plotted: 20:28 Jun 12, 1997

< EMI - ElectroMagnetic Instruments

Fig. 13a 
APPARENT RESISTIVITY

north of Beatty

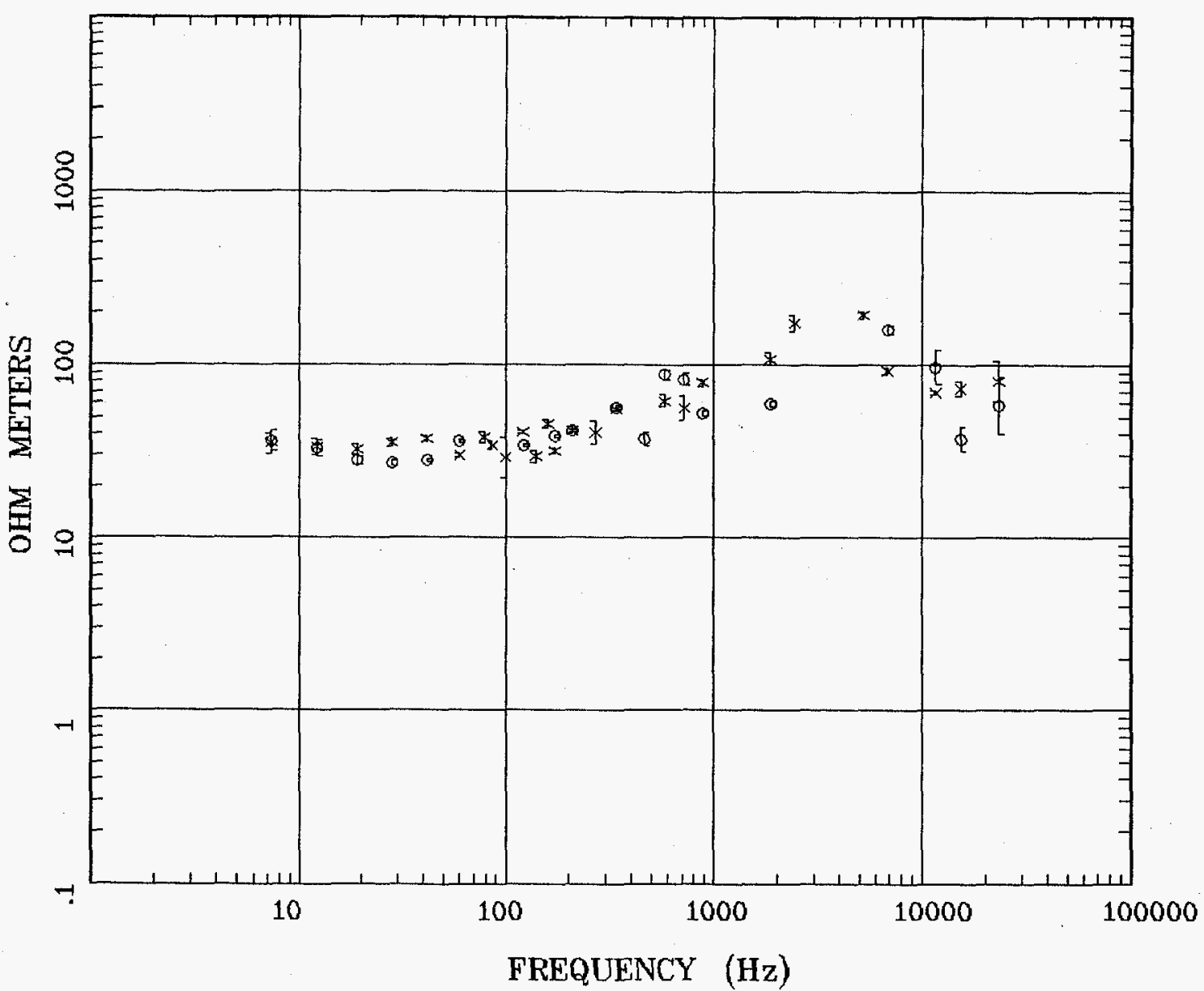

Client:

Rotation:

Filename: bba13comb.all

Remote: remote $\mathrm{e}$

Acquired: 20:0 Jun 12, 1997

Channels: Ch1 Ch2 Ch3 Ch4 Ch5 Ch6 Survey Co:USGS

Plotted: 20:28 Jun 12, 1997

< EMI - ElectroMagnetic Instruments

Fig. 13b 
IMPEDANCE PHASE north of Beatty

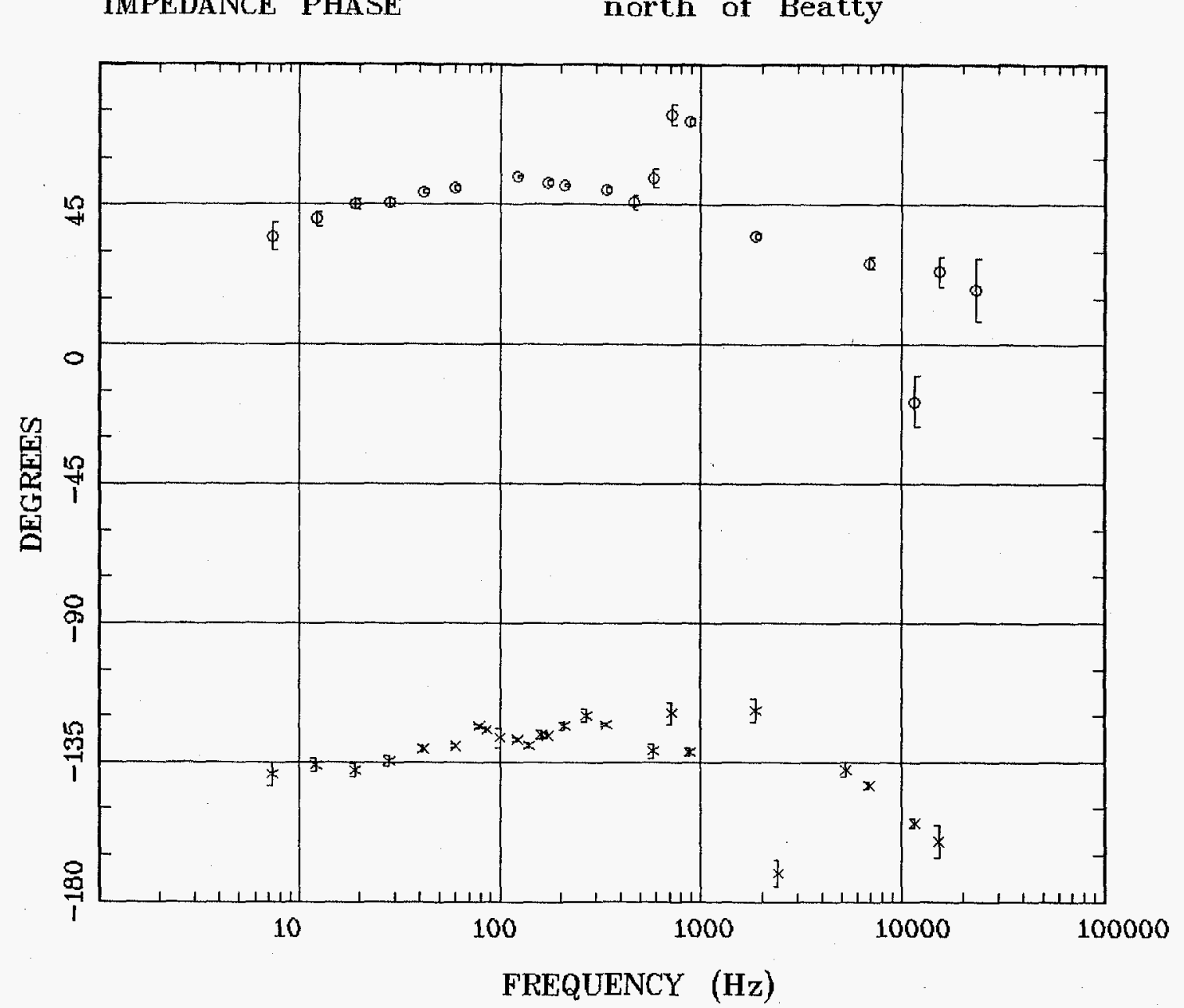

Client:

Remote: remote e

Acquired: 20:0 Jun 12, 1997 Survey Co:USGS
Rotation:

Filename: bba13comb.all

Channels: Ch1 Ch2 Ch3 Ch4 Ch5 Ch6

Plotted: 20:28 Jun 12, 1997

< EMI - ElectroMagnetic Instruments

Fig. 13c 


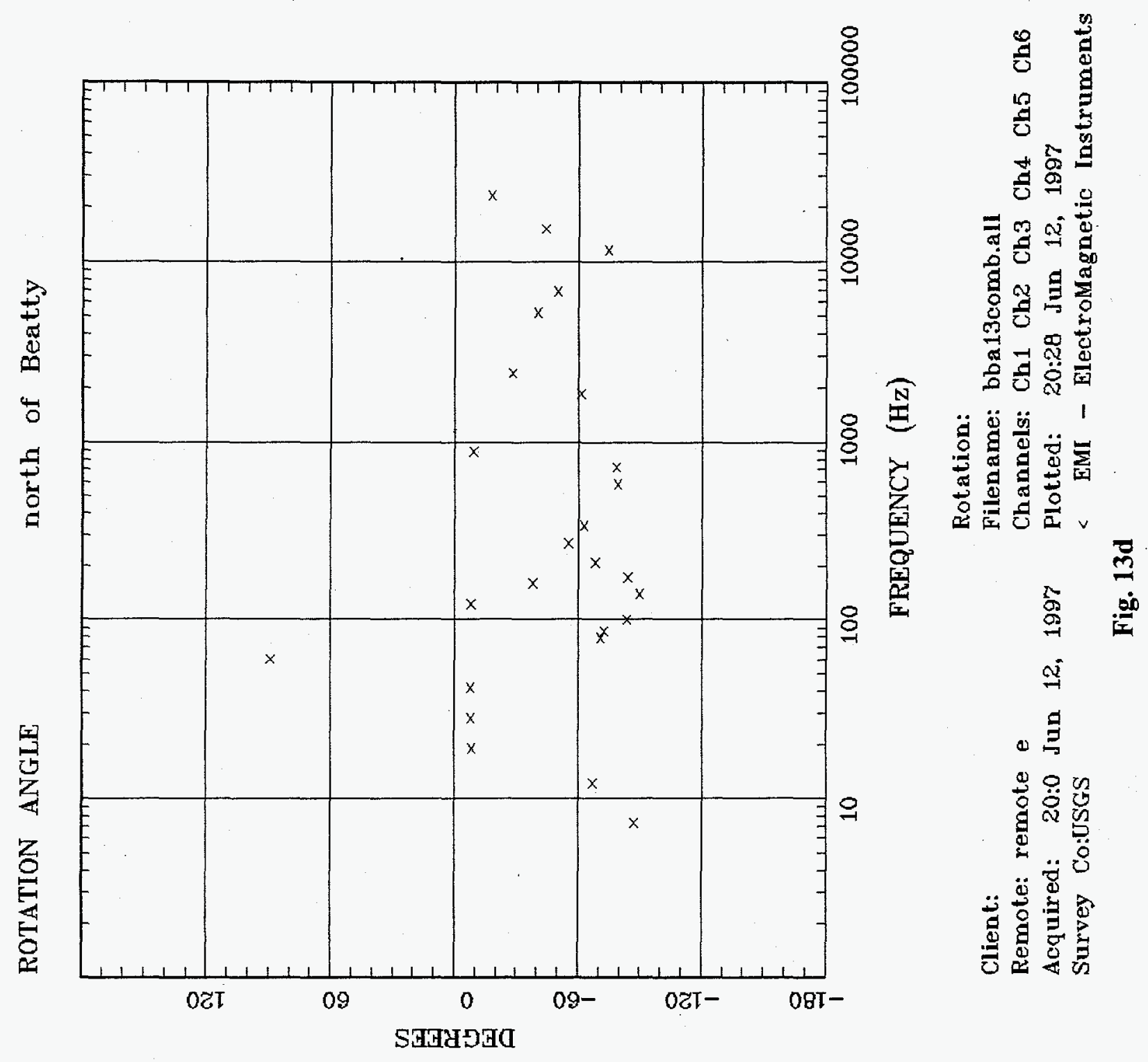




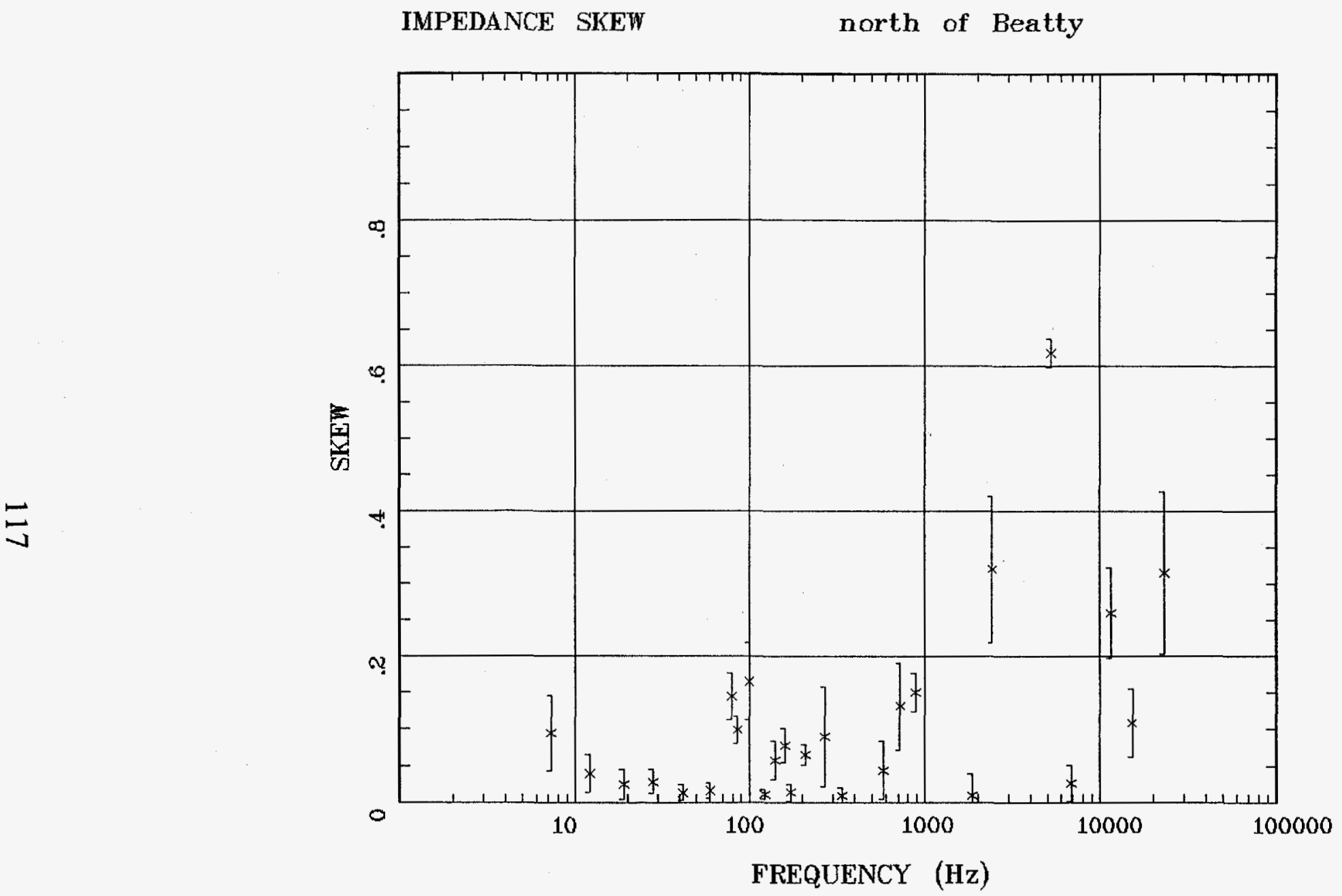

Client:

Rotation:

Remote: remote e

Acquired: 20:0 Jun 12, 1997

Filename: bba13comb.all

Channels: Ch1 Ch2 Ch3 Ch4 Ch5 Ch6 Survey Co:USGS

Plotted: 20:28 Jun 12, 1997

$<$ EMI - ElectroMagnetic Instruments

Fig. 13e 
north of Beatty

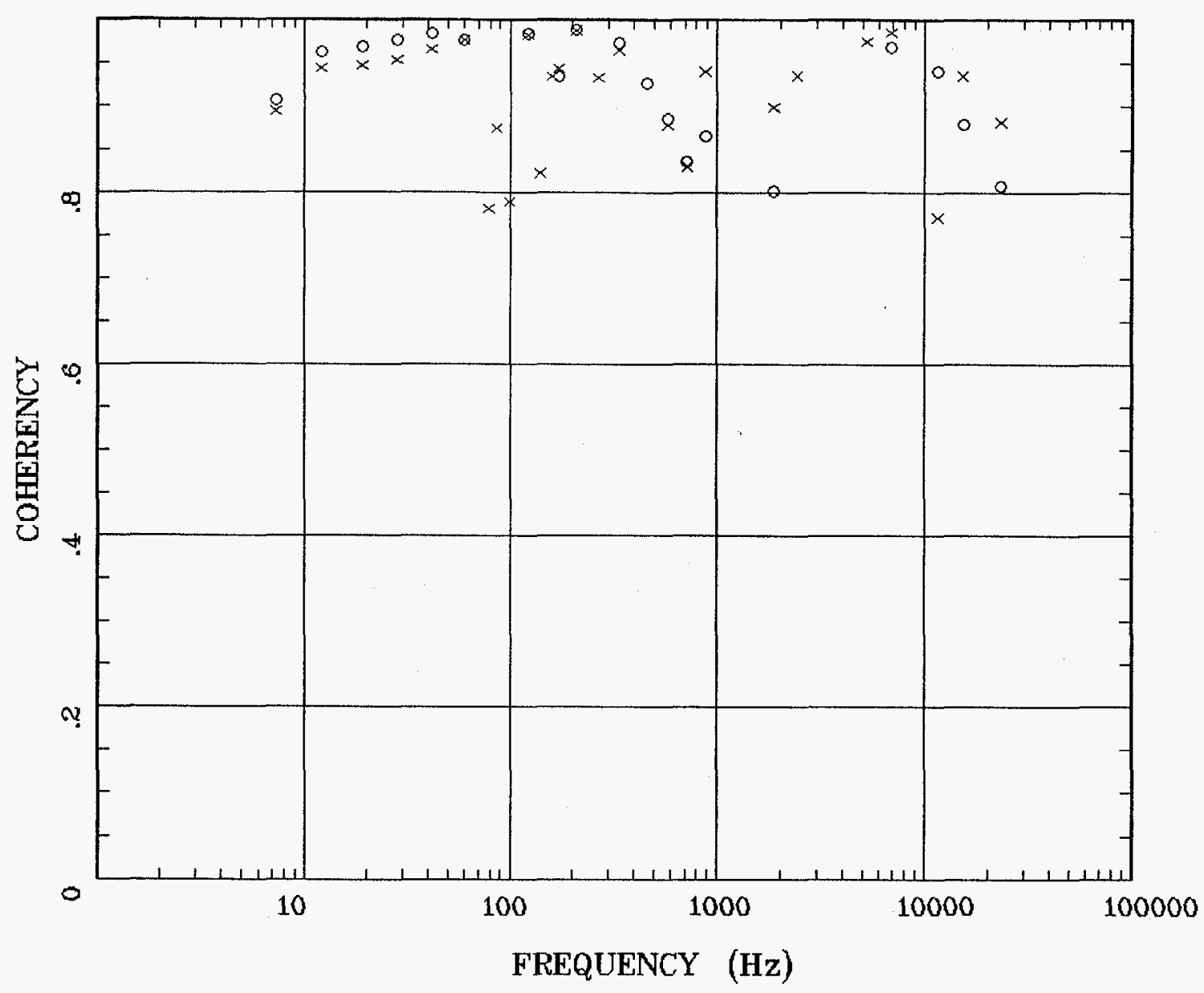

Client:

Remote: remote e

Acquired: 20:0 Jun 12, 1997 Survey Co:USGS

\section{Rotation:}

Filename: bba13comb.all

Channels: Ch1 Ch2 Ch3 Ch4 Ch5 Ch6

Plotted: 20:28 Jun 12, 1997

< EMI - ElectroMagnetic Instruments

Fig. 13f 
north of Beatty

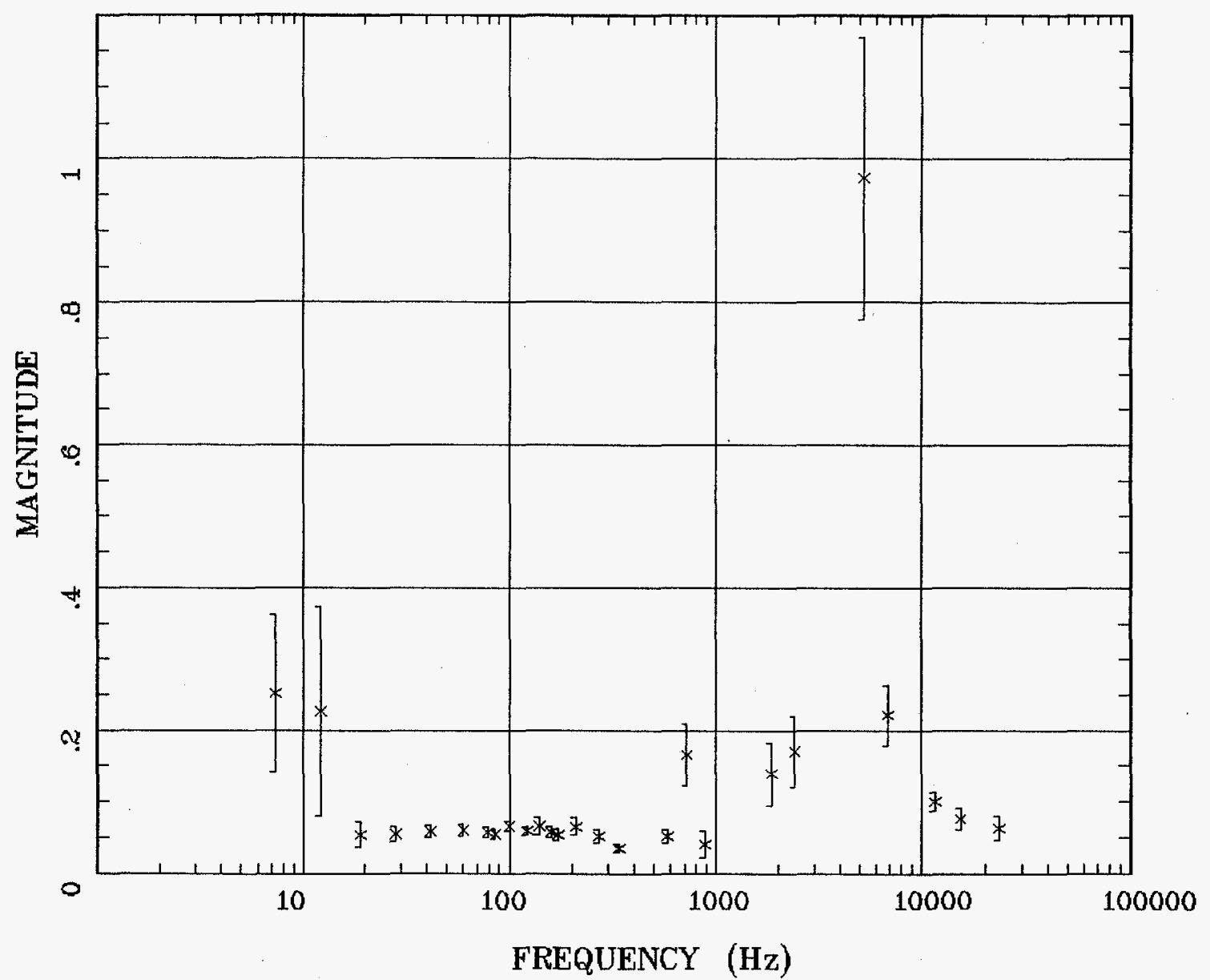

Client:

Remote: remote e Acquired: 20:0 Jun 12, 1997 Survey Co:USGS
Rotation:

Filename: bba13comb.all

Channels: Ch1 Ch2 Ch3 Ch4 Ch5 Ch6

Plotted: 20:29 Jun 12, 1997

< EMI - ElectroMagnetic Instruments

Fig. 13g 
north of Beatty

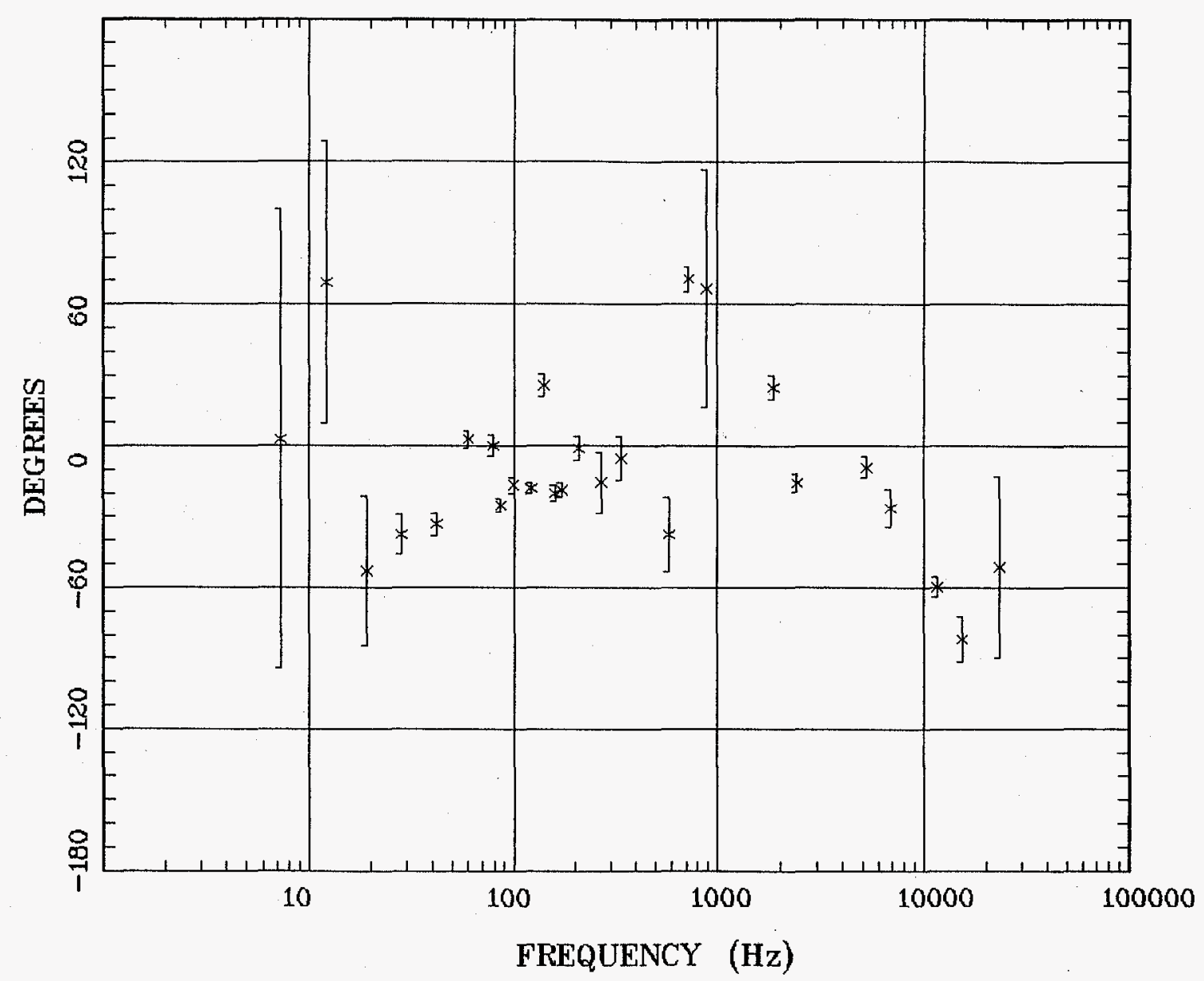

Client:

Remote: remote e

Acquired: 20:0 Jun 12, 1997 Survey Co:USGS
Rotation:

Filename: bba13comb.all

Channels: Ch1 Ch2 Ch3 Ch4 Ch5 Ch6

Plotted: 20:29 Jun 12, 1997

< EMI - ElectroMagnetic Instruments

Fig. 13h 


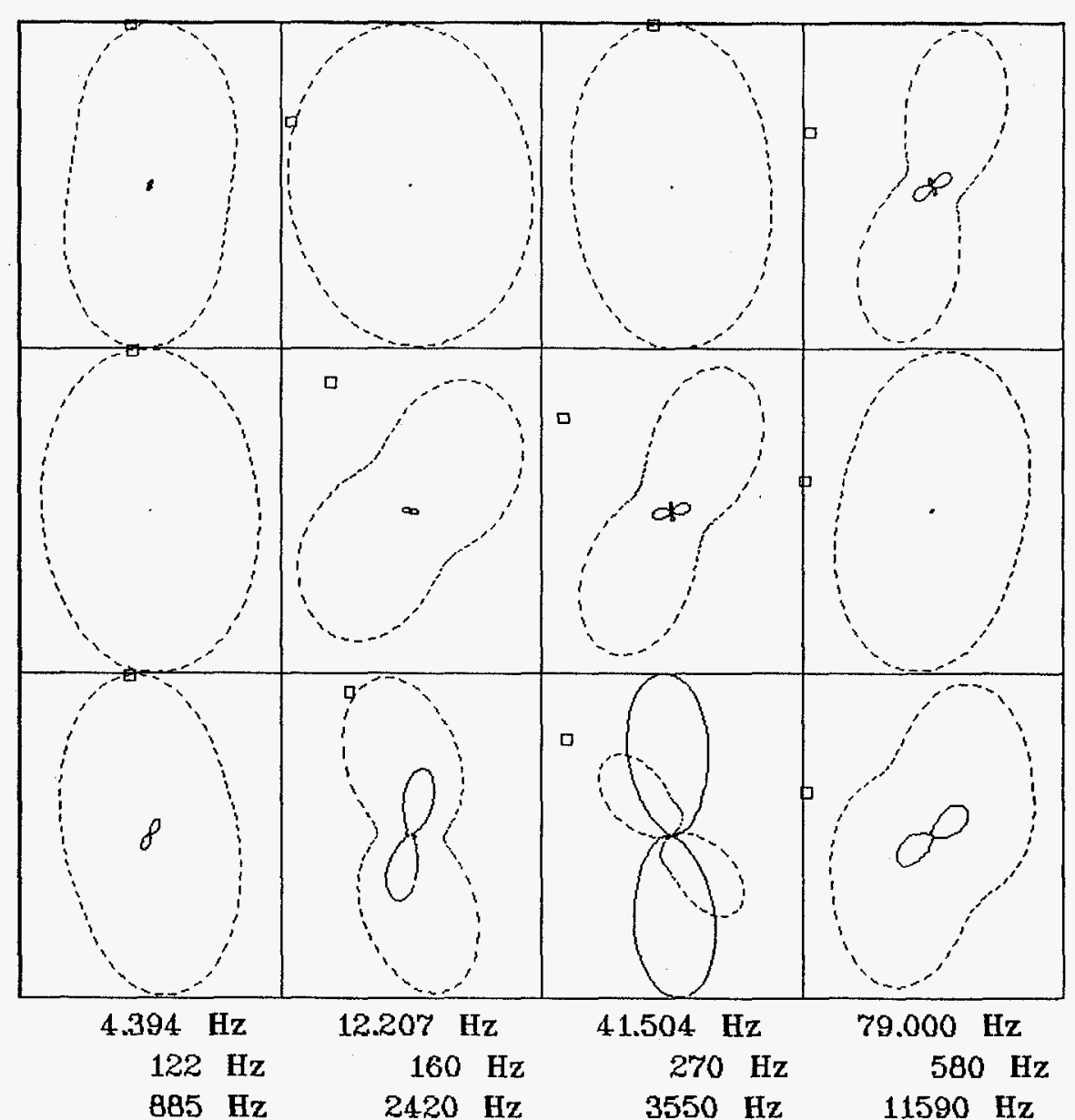

Client:

Rotation:

Remote: remote e

Filename: bba13comb.all

Acquired: 20:0 Jun

Channels: Ch1 Ch2 Ch3 Ch4 Ch5 Ch6 Ch7 Survey Co:USGS

Plotted: 20:29 Jun 12, 1997

Fig. 13i 


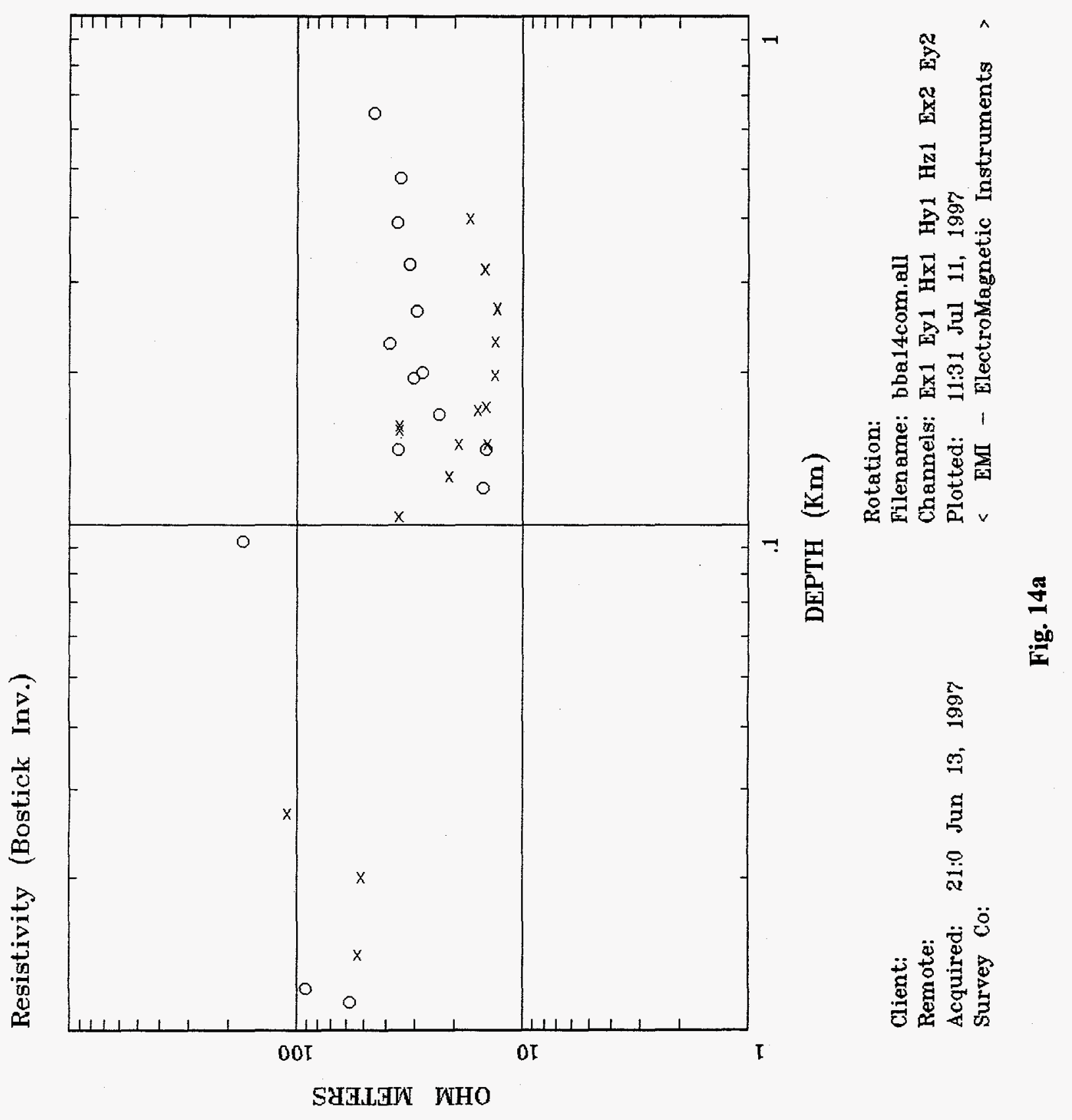


APPARENT RESISTIVITY

north of Beatty

$\omega$

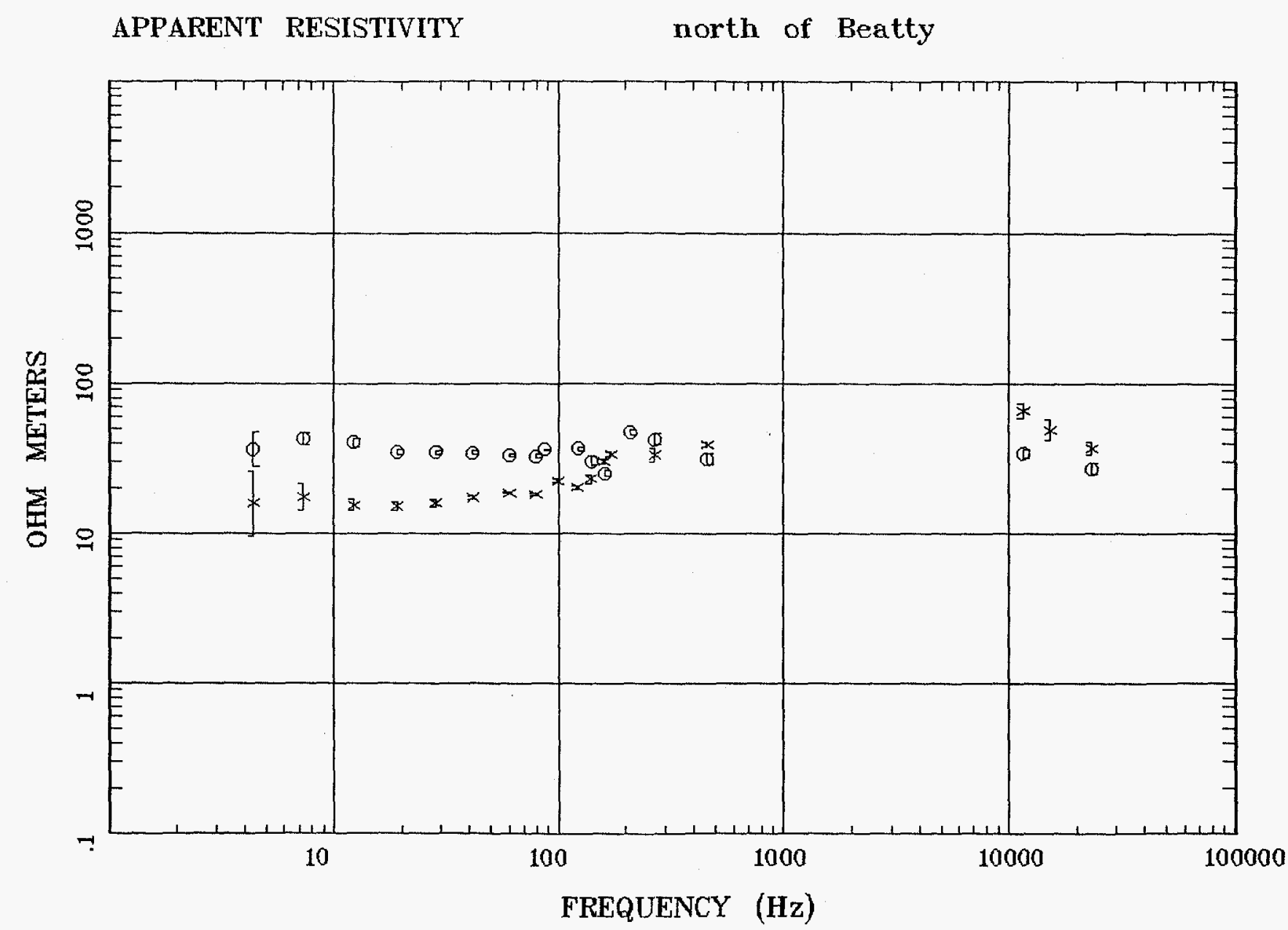

Client:

Remote: remote $\mathrm{e}$

Acquired: 21:0 Jun 13, 1997 Survey Co:US Geological Survey

\section{Rotation:}

Filename: bba14com.all

Channels: Ch1 Ch2 Ch3 Ch4 Ch5 Ch6 Ch7

Plotted: 11:31 Jul 11, 1997

< EMI - ElectroMagnetic Instruments >

Fig. 14b 


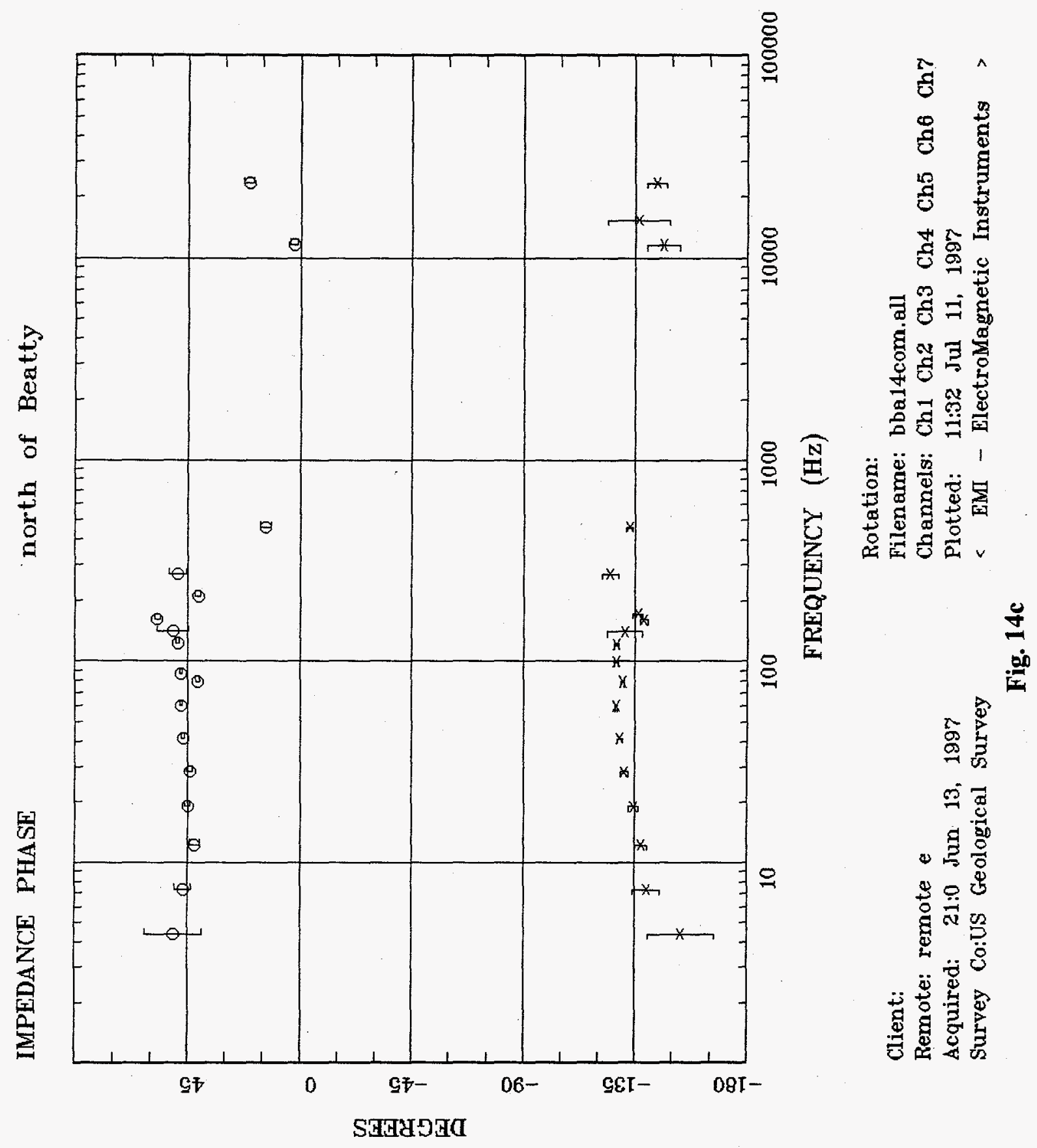




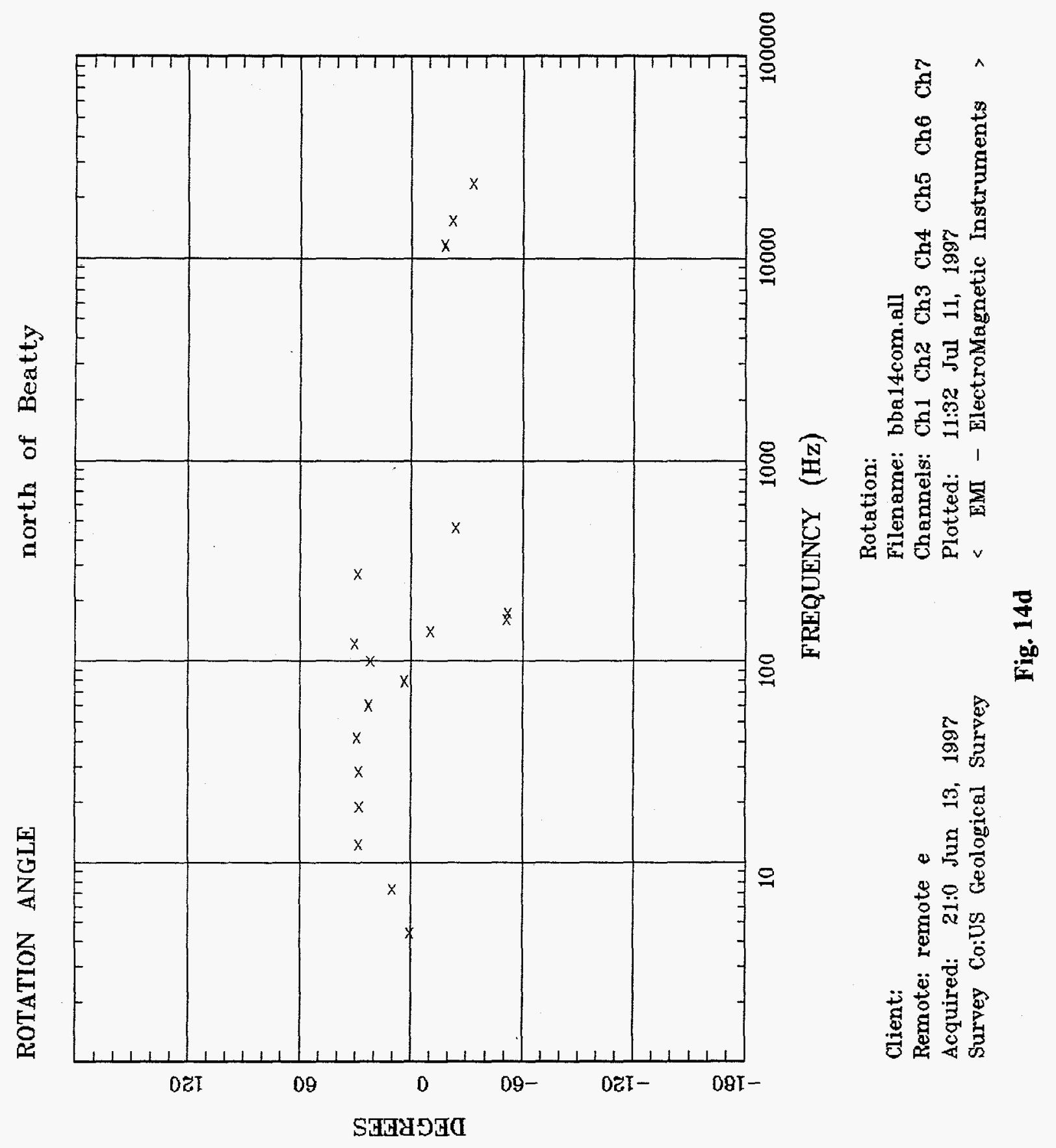




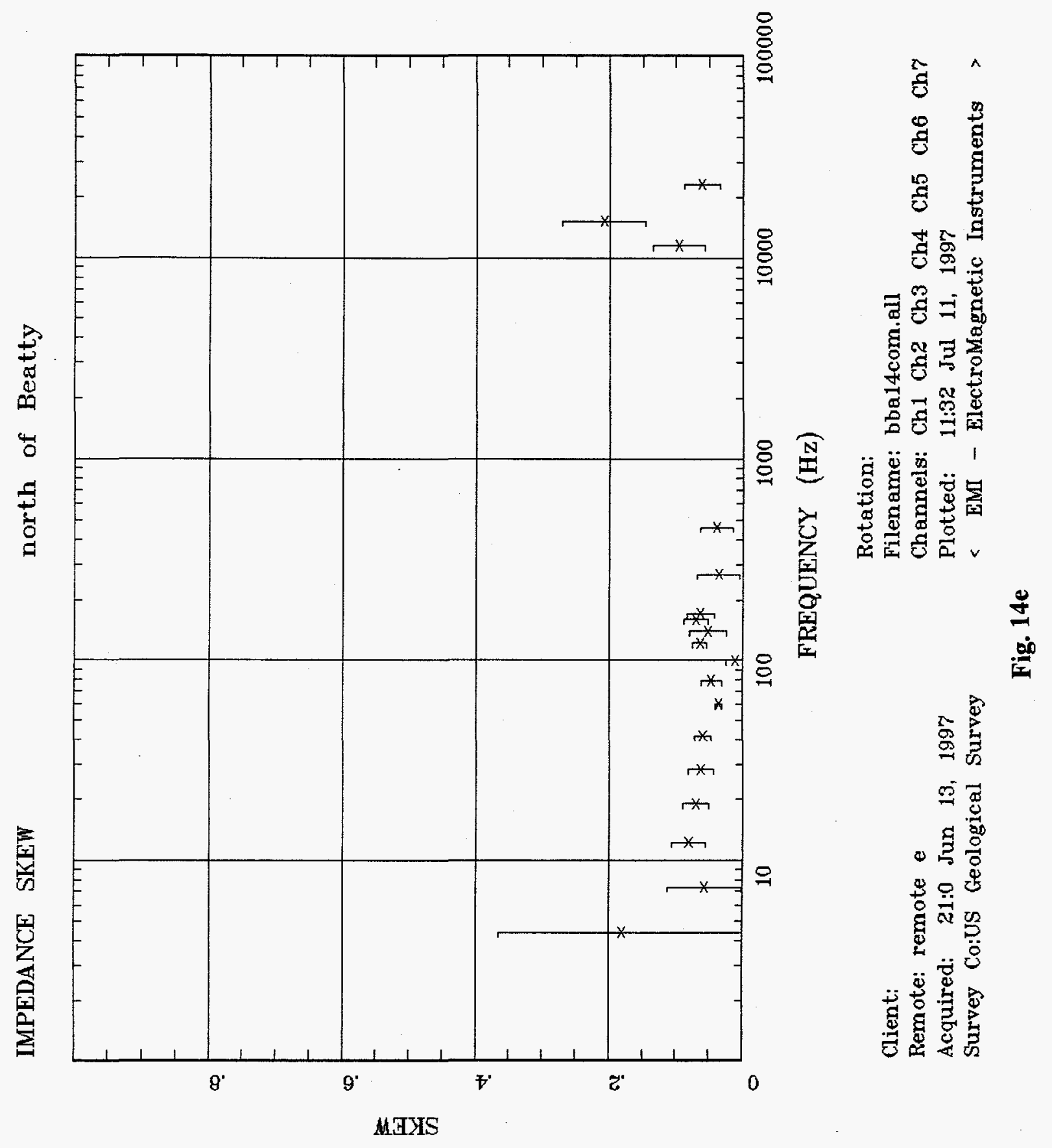




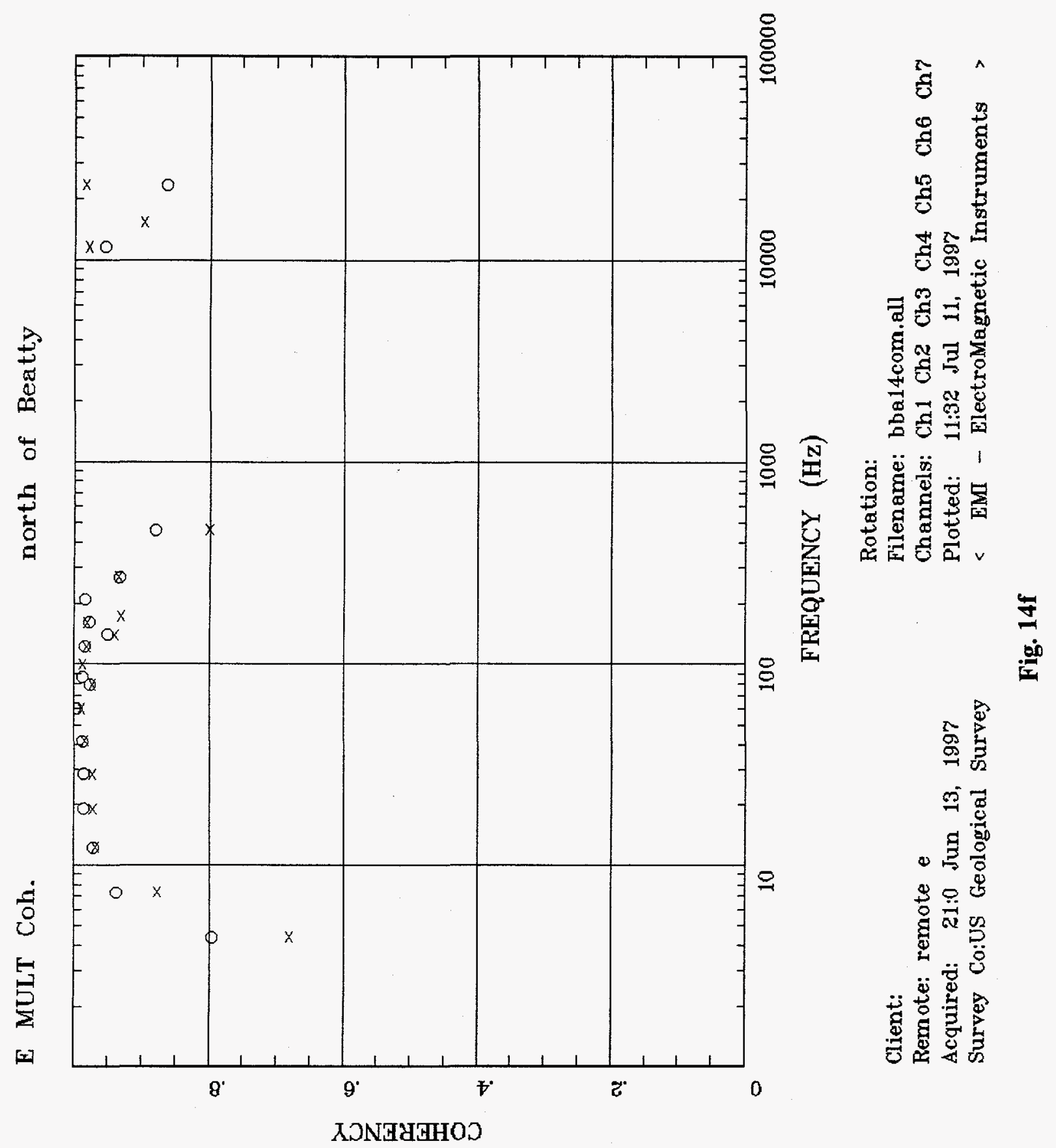


$8 Z I$

MAGNITUDE

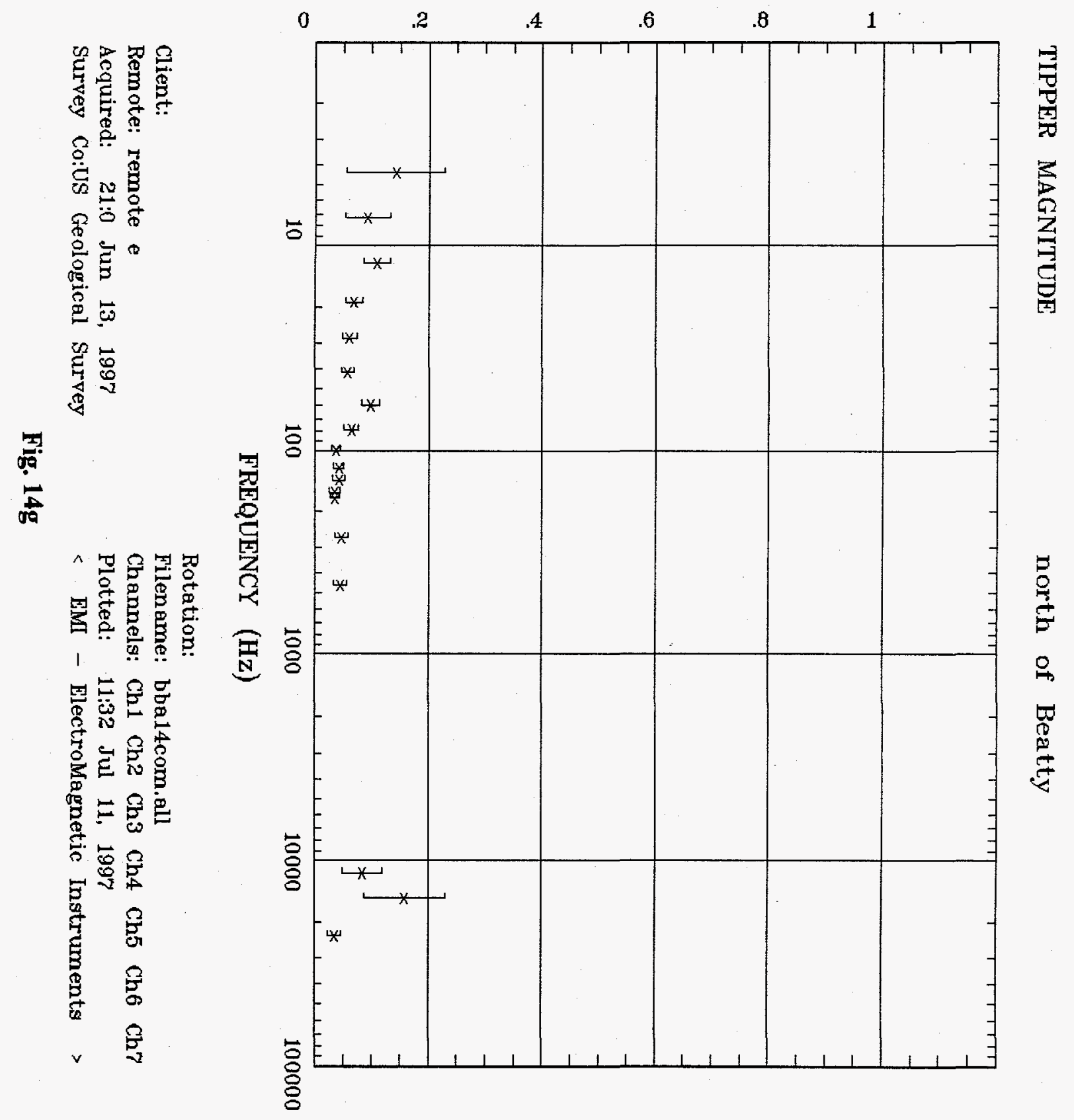




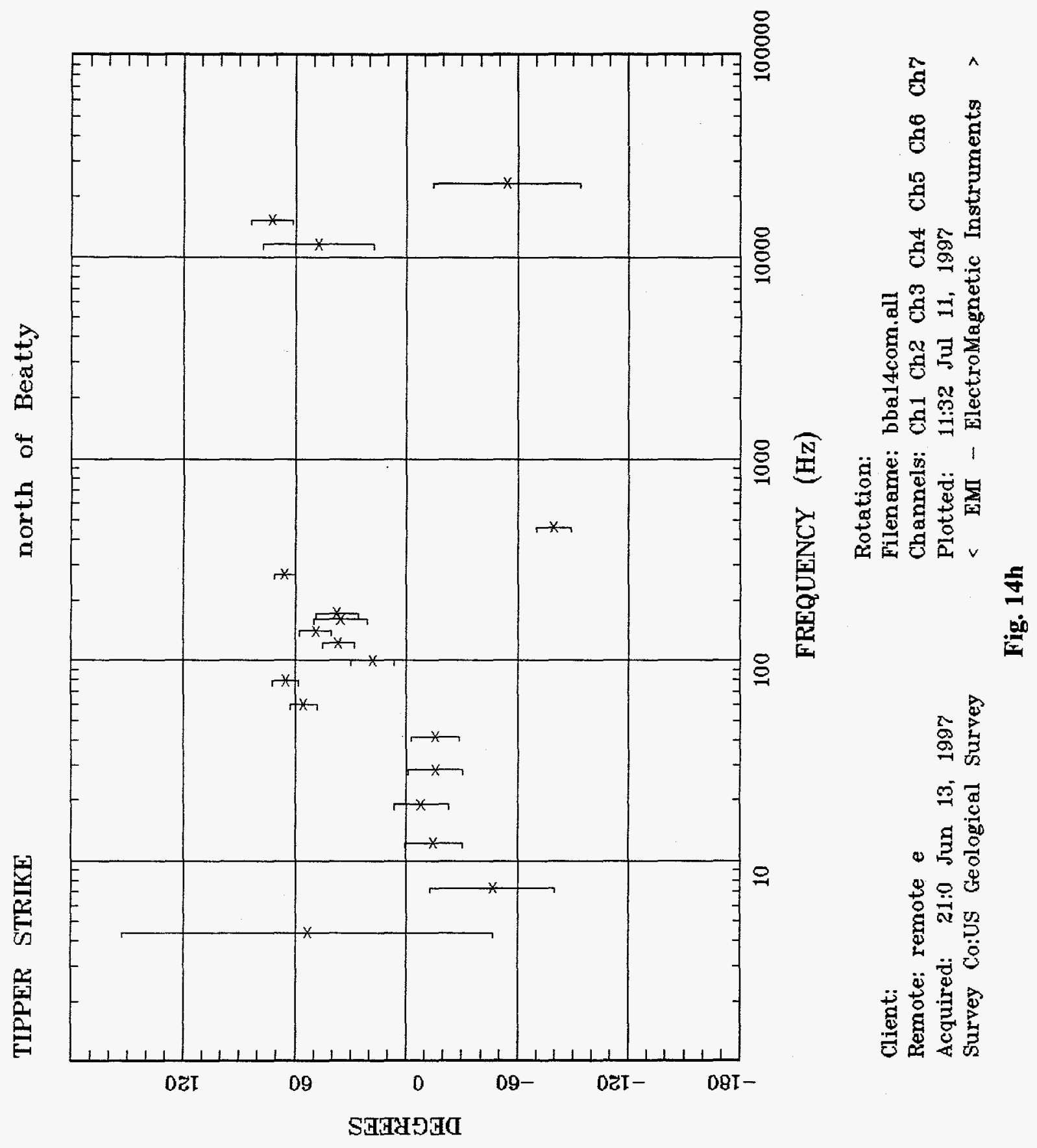




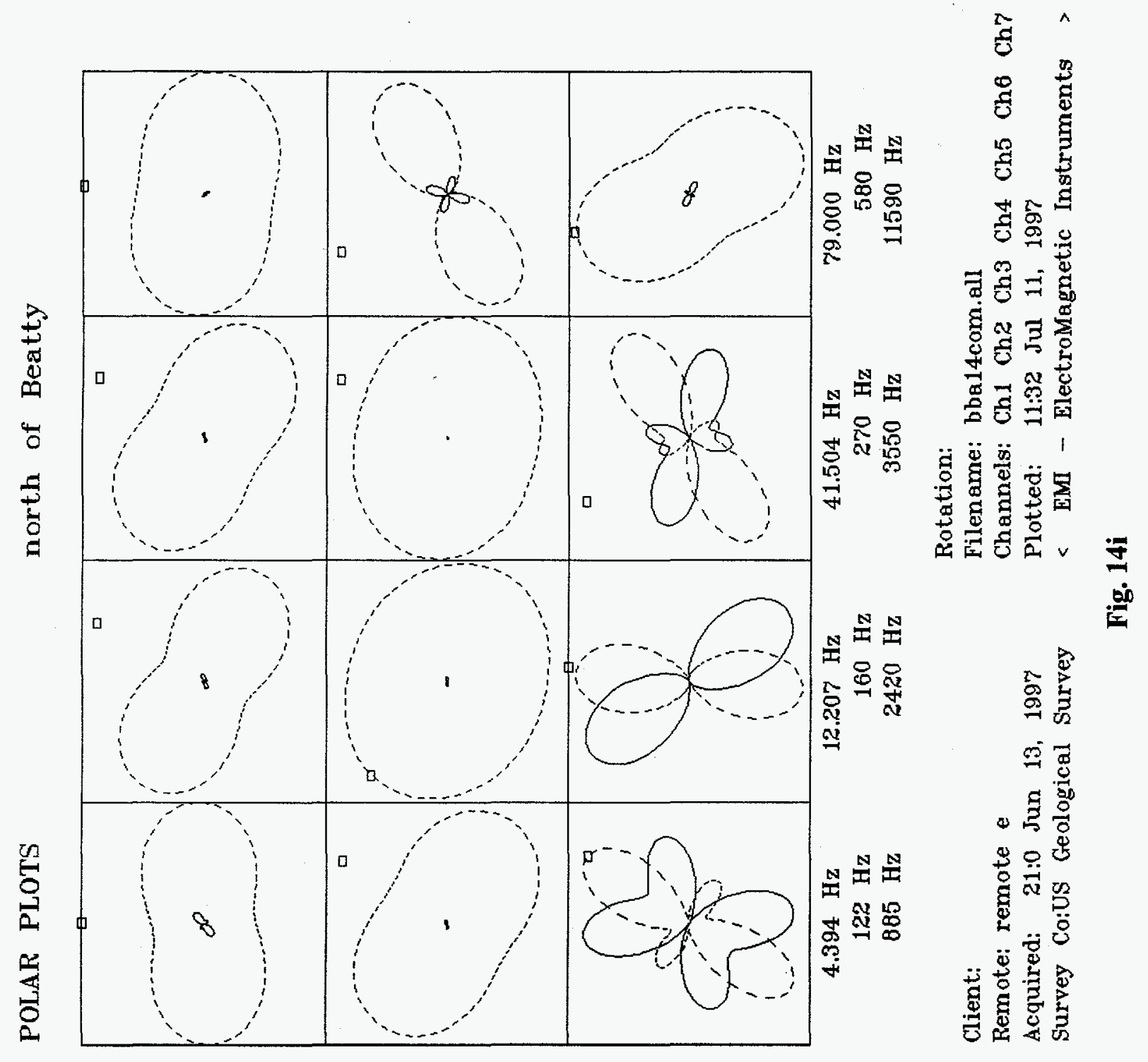




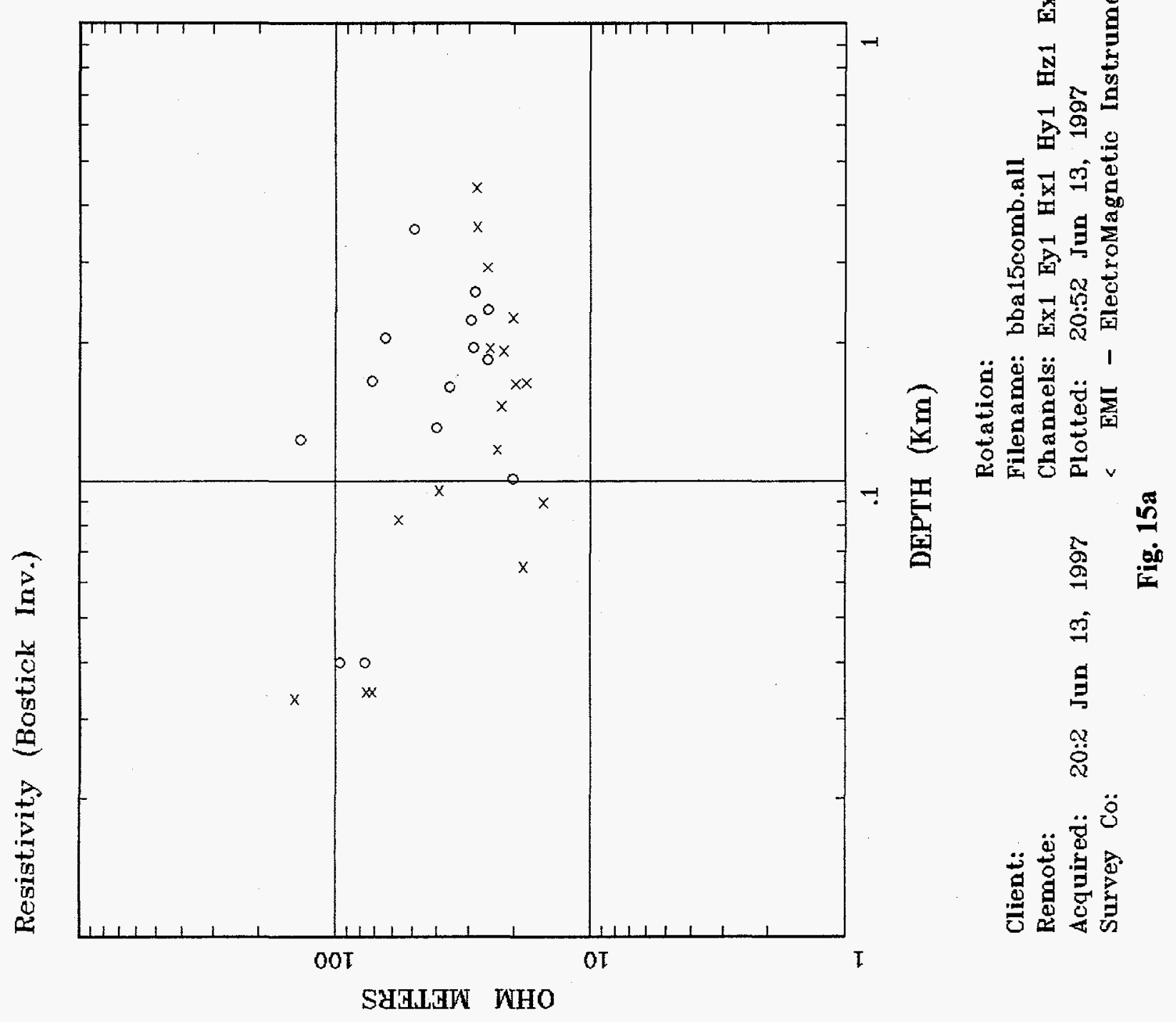




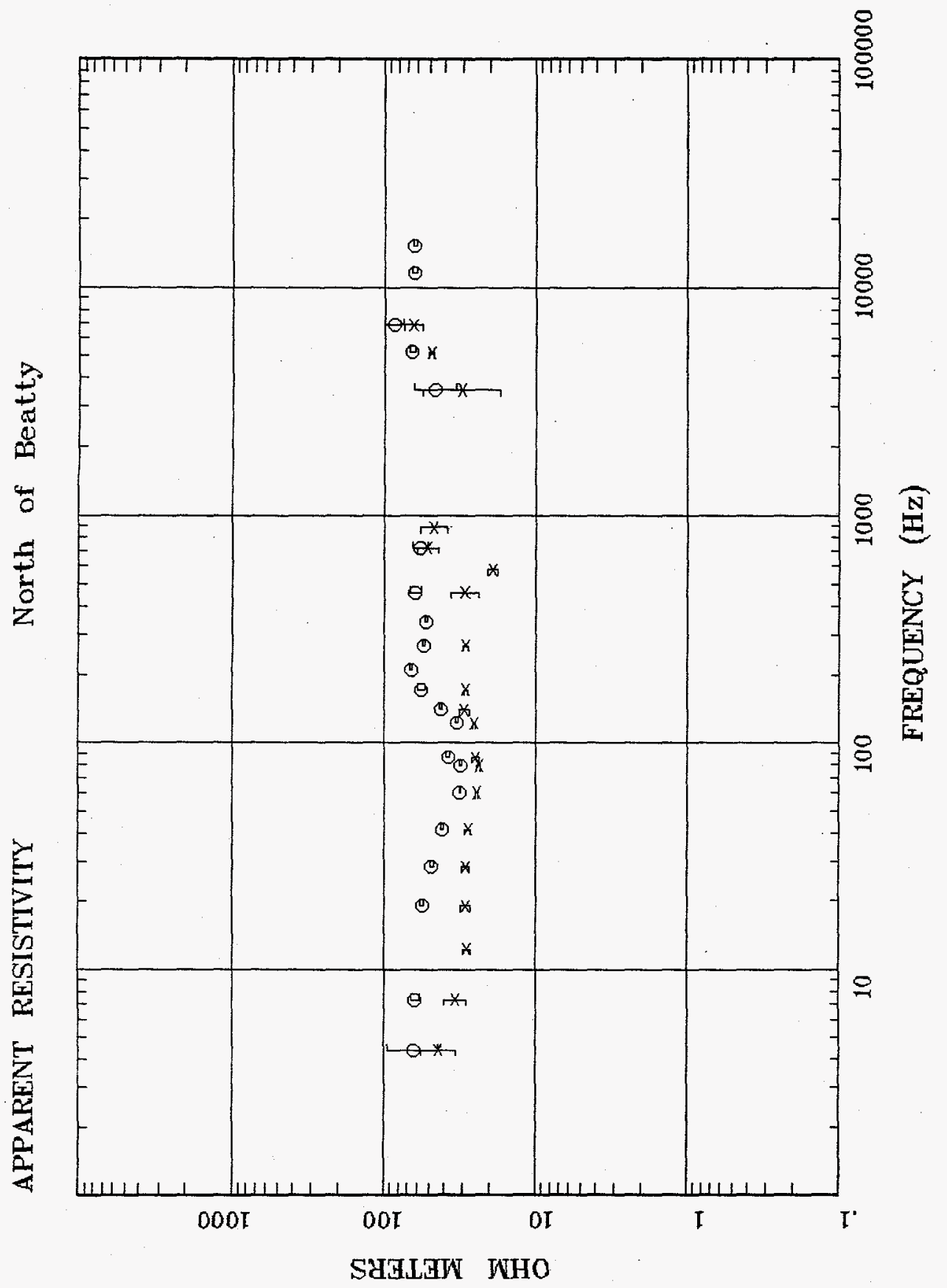

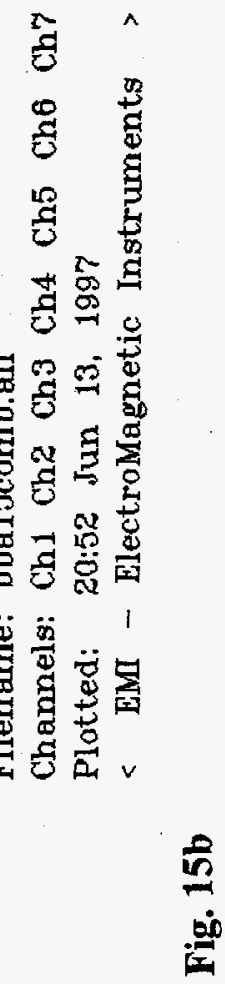

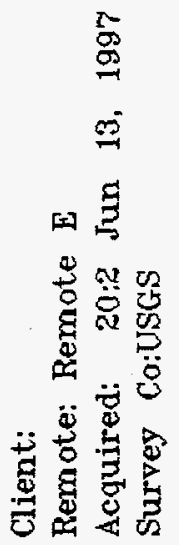


IMPEDANCE PHASE

North of Beatty

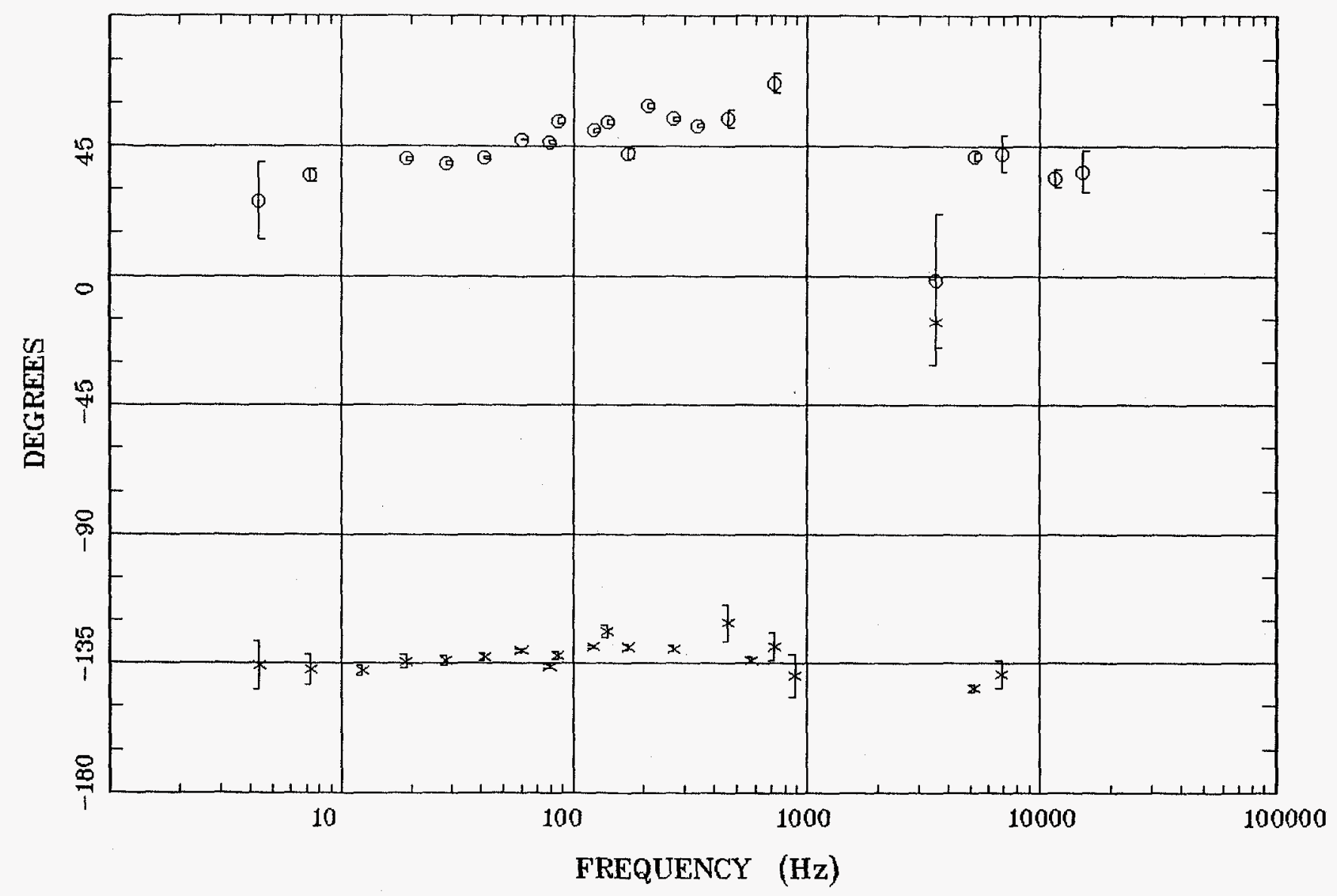

Client:

Rotation:

Remote: Remote E

Acquired: 20:2 Jun 13, 1997

Survey Co:USGS

Filename: bba15comb.all

Channels: Ch1 Ch2 Ch3 Ch4 Ch5 Ch6 Ch7

Plotted: 20:52 Jun 13, 1997

< EMI - ElectroMagnetic Instruments >

Fig. 15c 
ROTATION ANGLE

North of Beatty

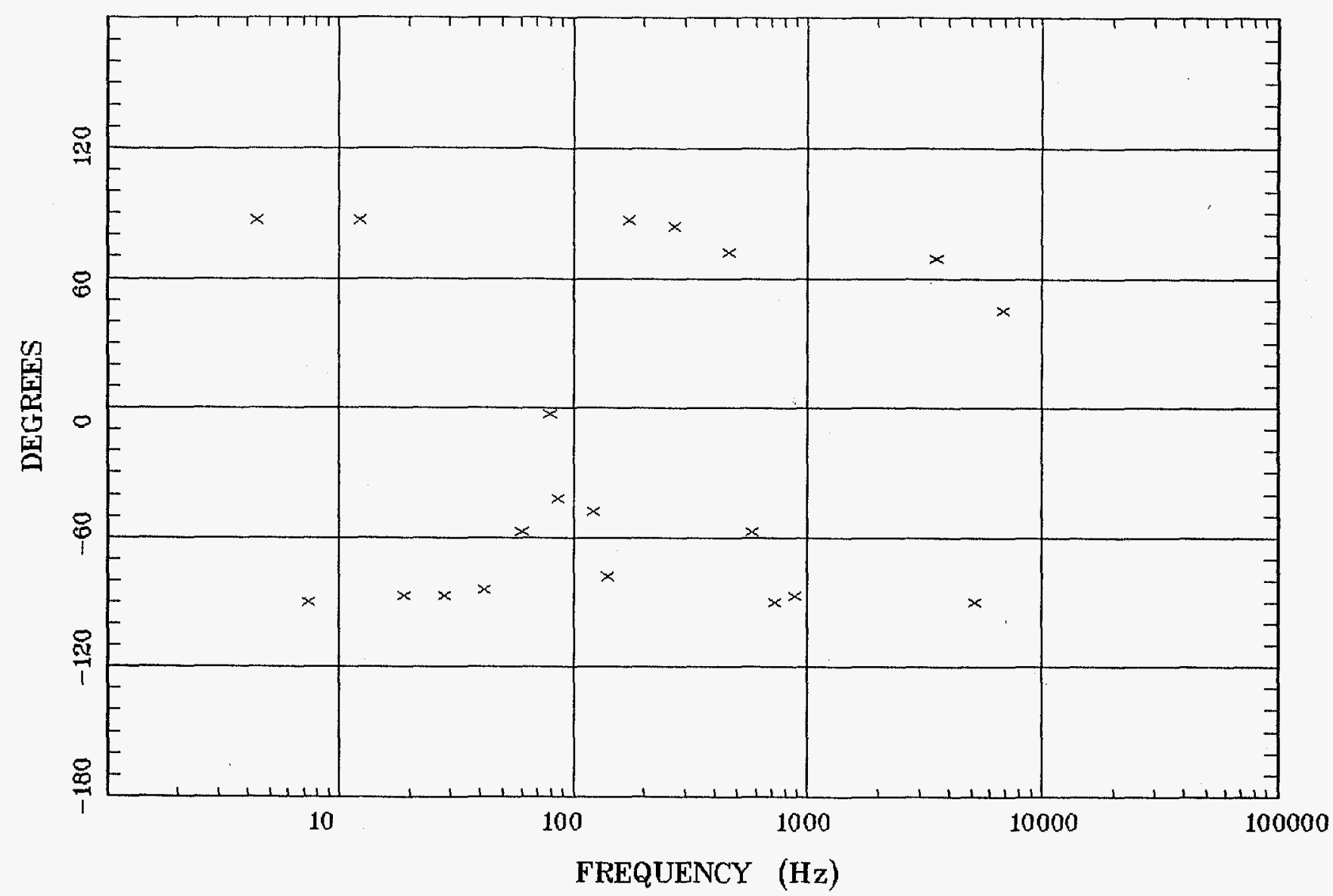

Client:

Remote: Remote $\mathrm{E}$

Acquired: 20:2 Jun 13, 1997

Rotation:

Filename: bba15comb.all

Channels: Ch1 Ch2 Ch3 Ch4 Ch5 Ch6 Ch7

Plotted: 20:52 Jun 13, 1997

Survey Co:USGS

< EMI - ElectroMagnetic Instruments >

Fig. 15d 


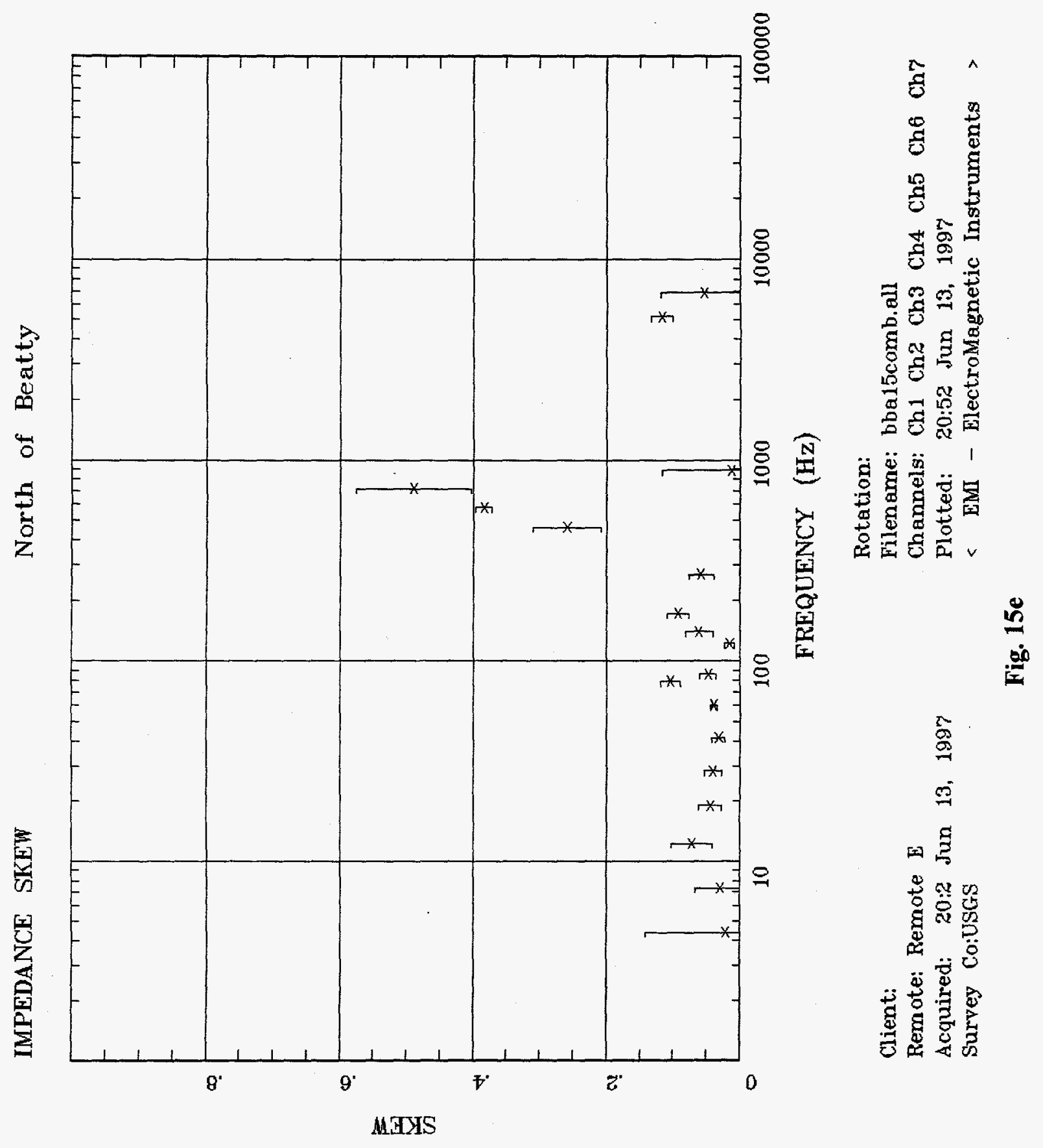




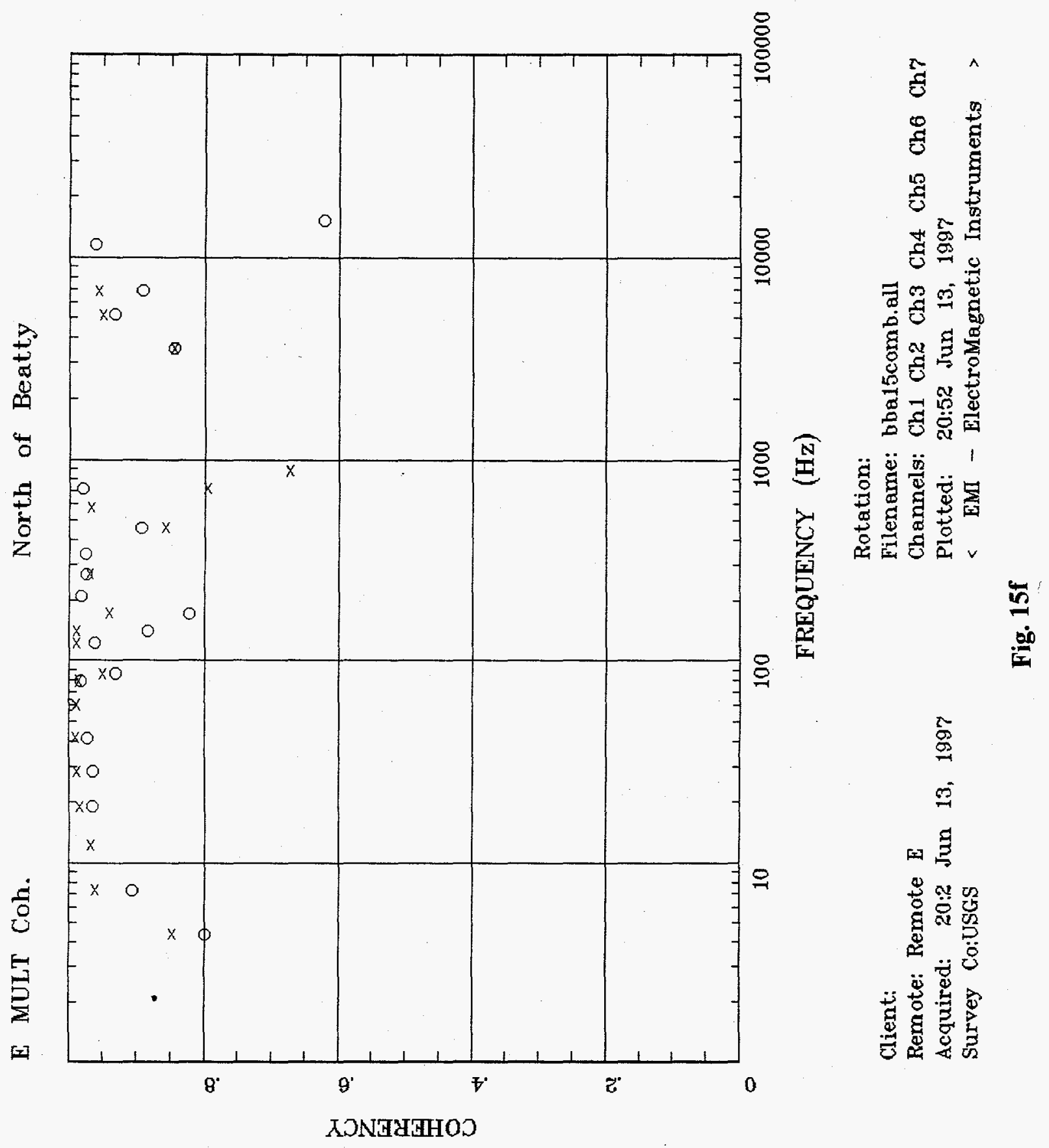



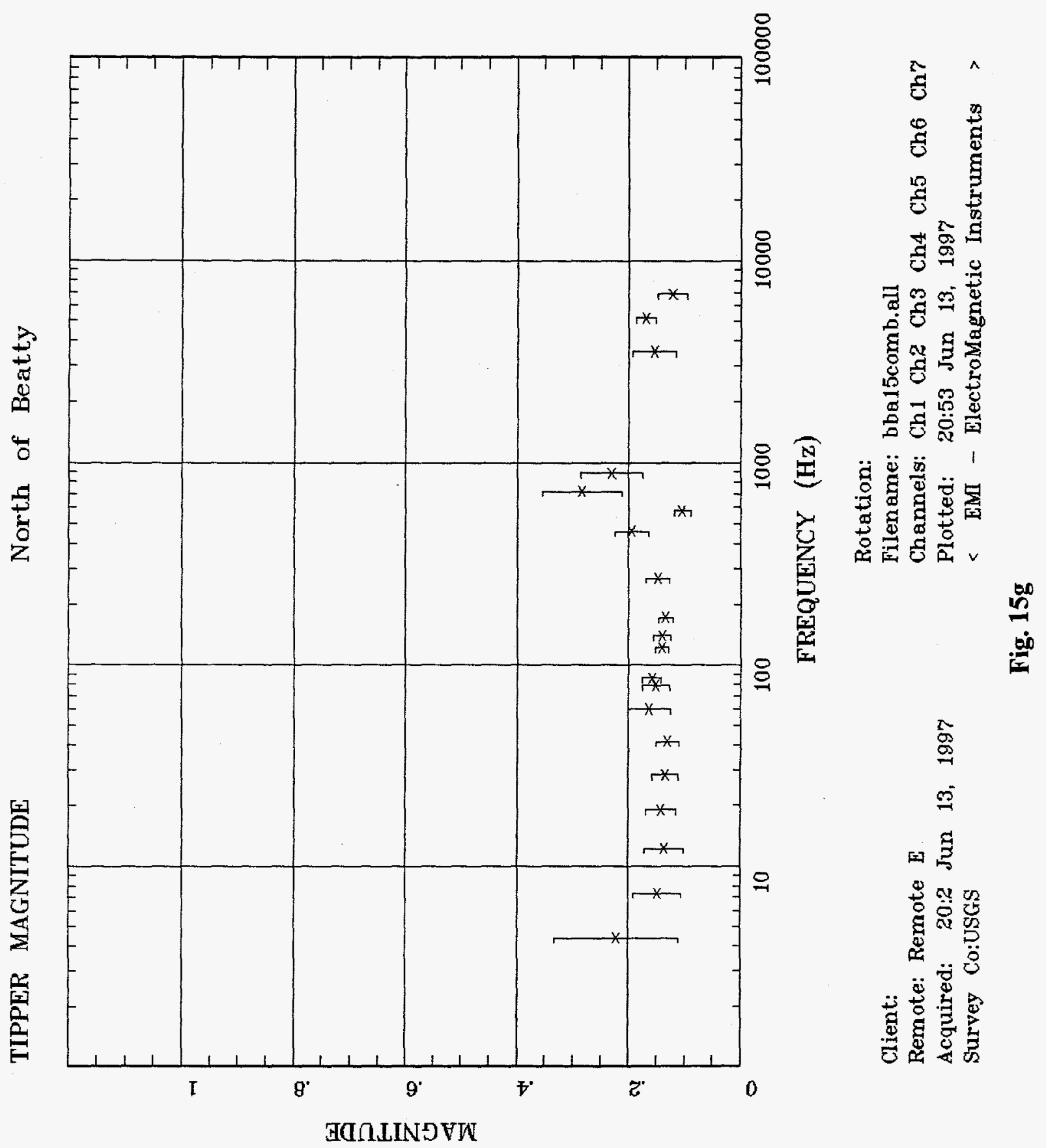


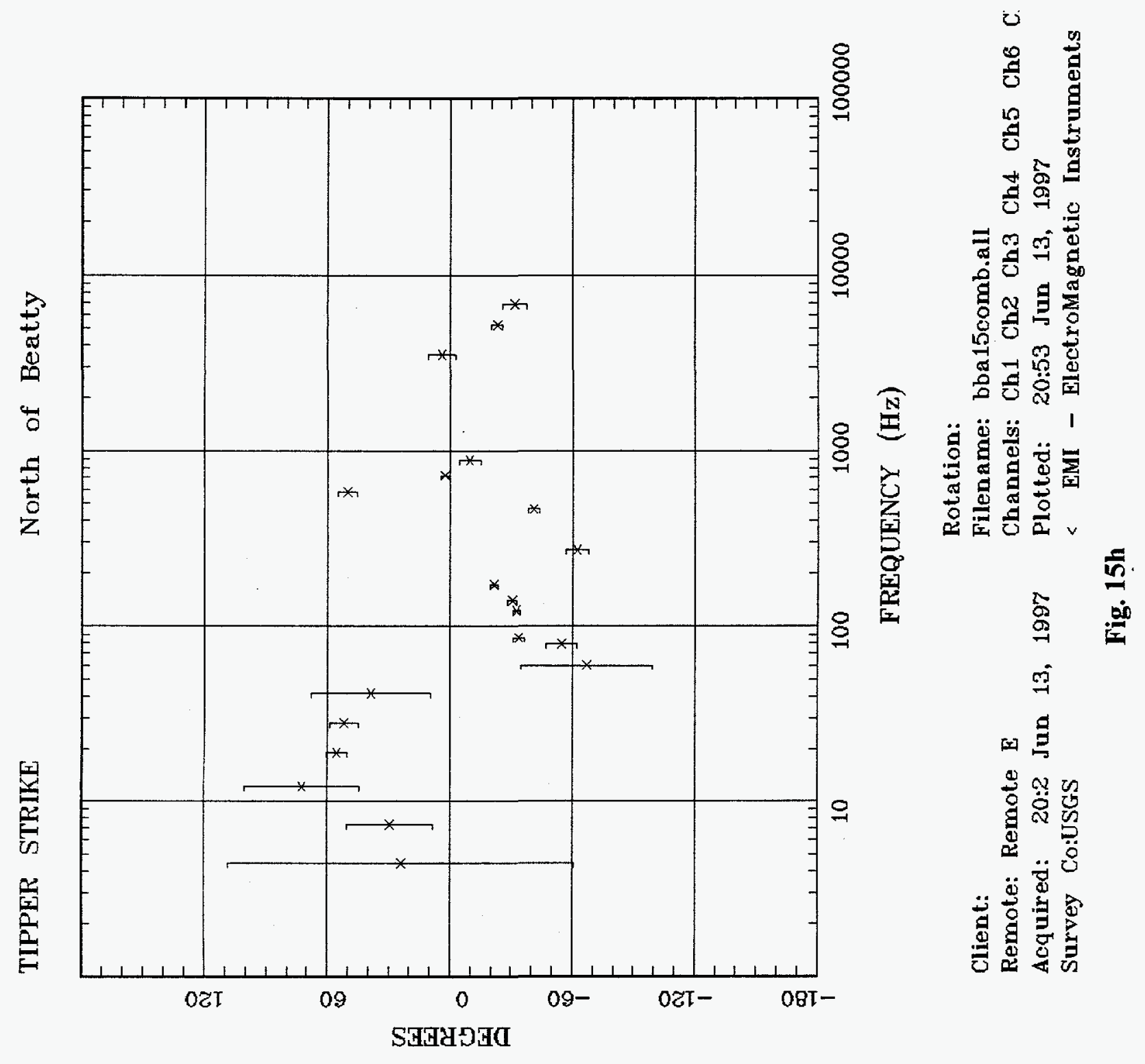




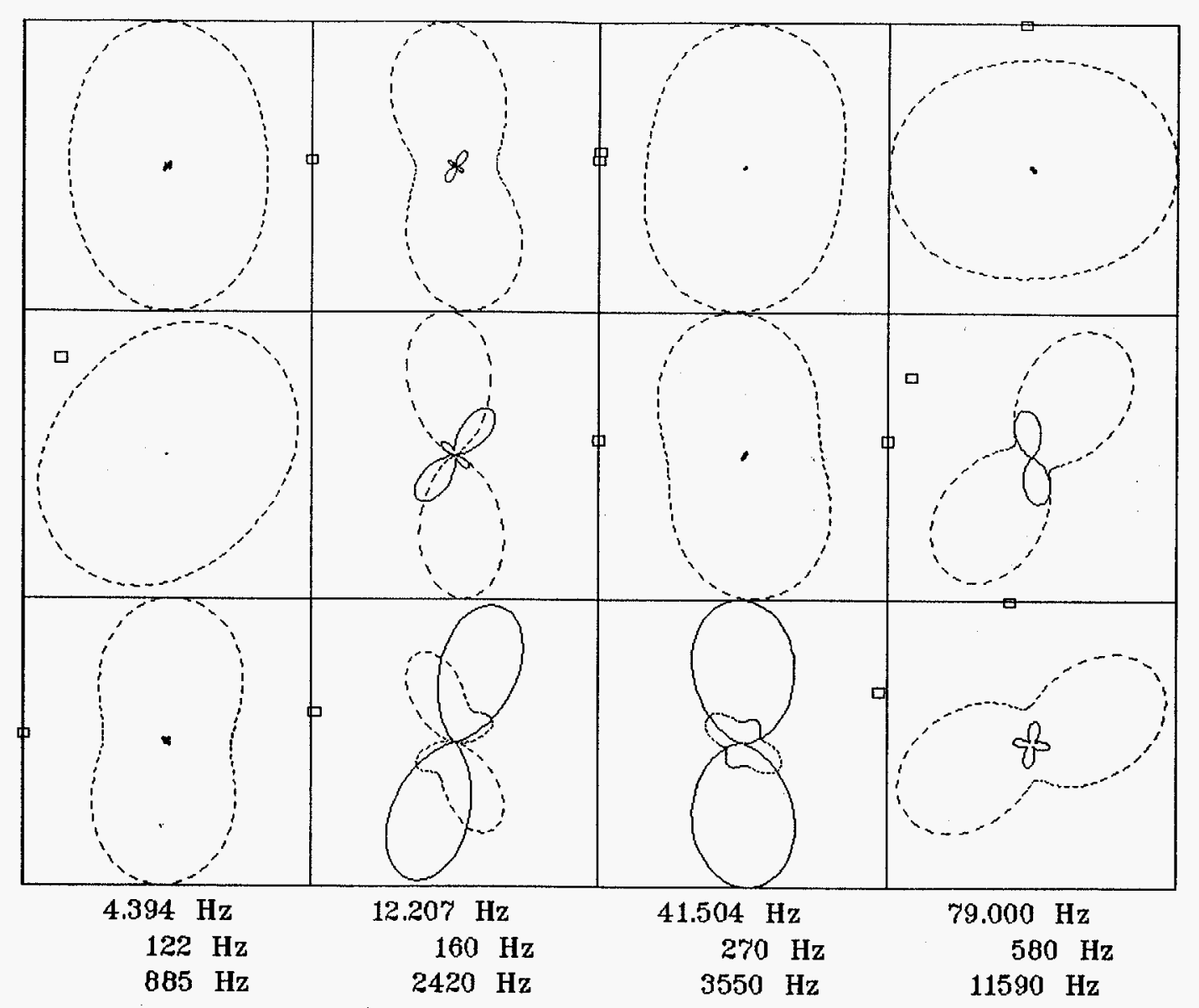

\section{Client:}

Remote: Remote E

Acquired: 20:2 Jun 13, 1997 Survey Co:USGS

Rotation:

Filename: bba15comb.all

Channels: Ch1 Ch2 Ch3 Ch4 Ch5 Ch6 Ch7

Plotted: 20:53 Jun 13, 1997

< EMI - ElectroMagnetic Instruments >

Fig: 15i 


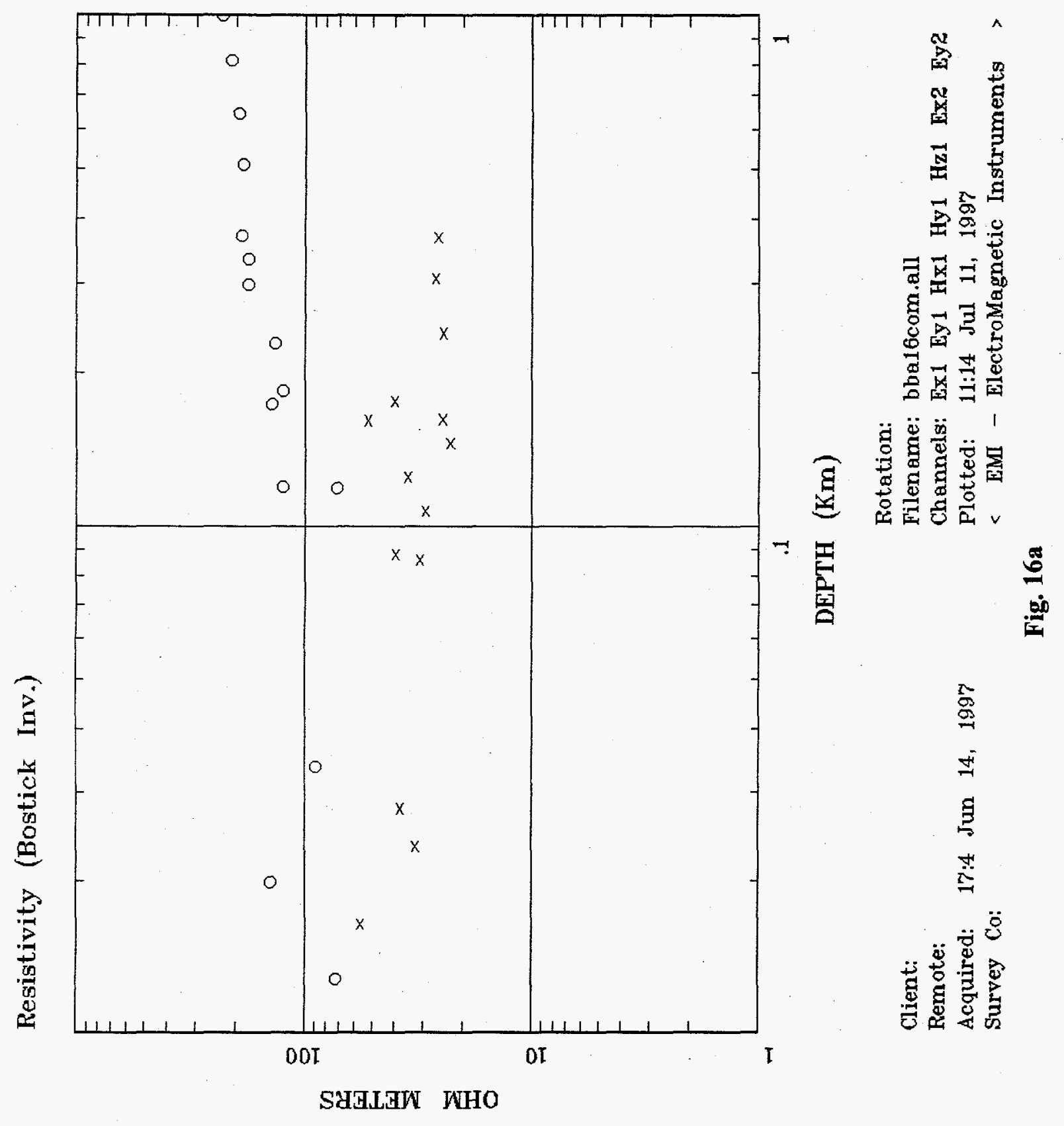




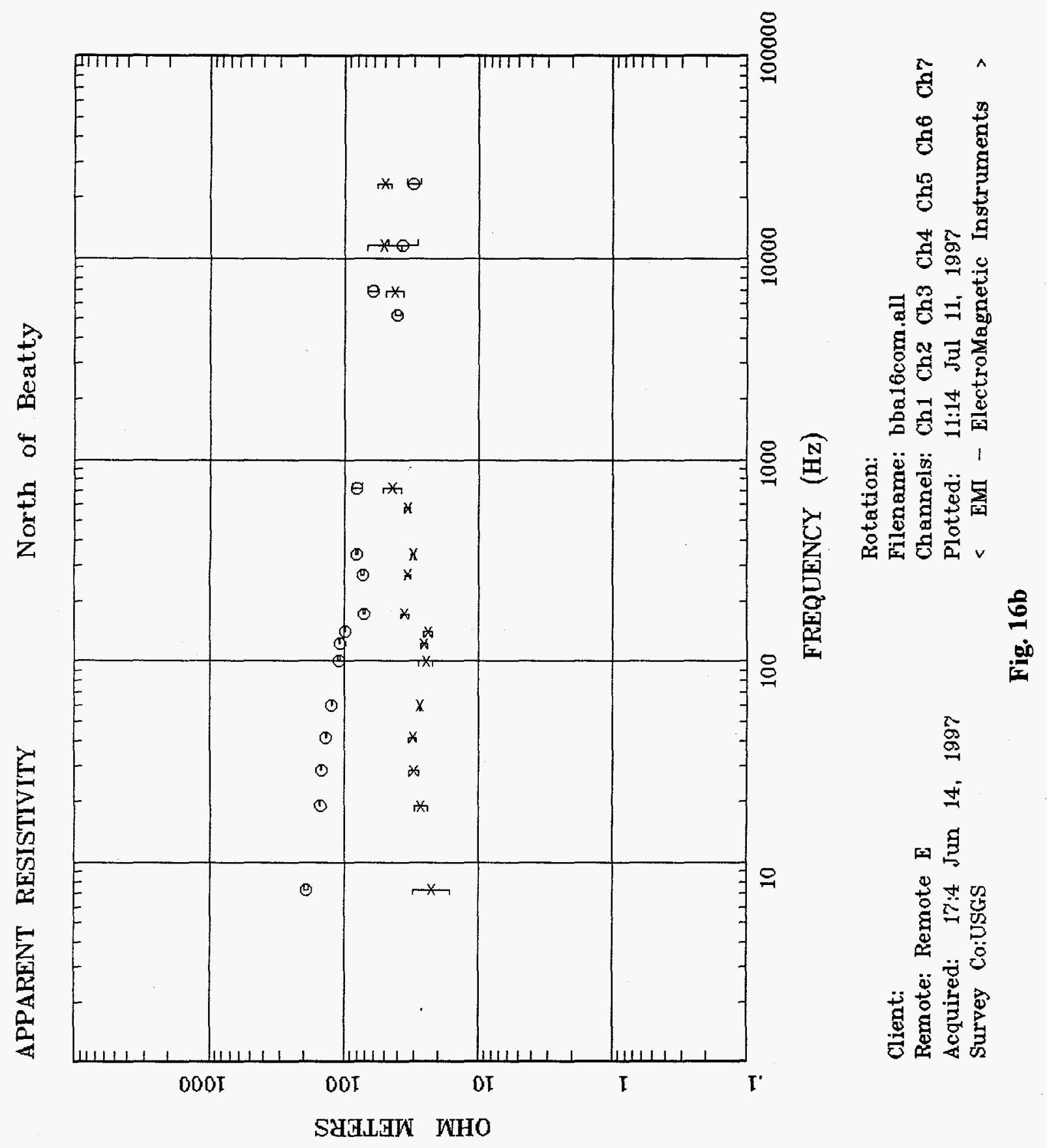




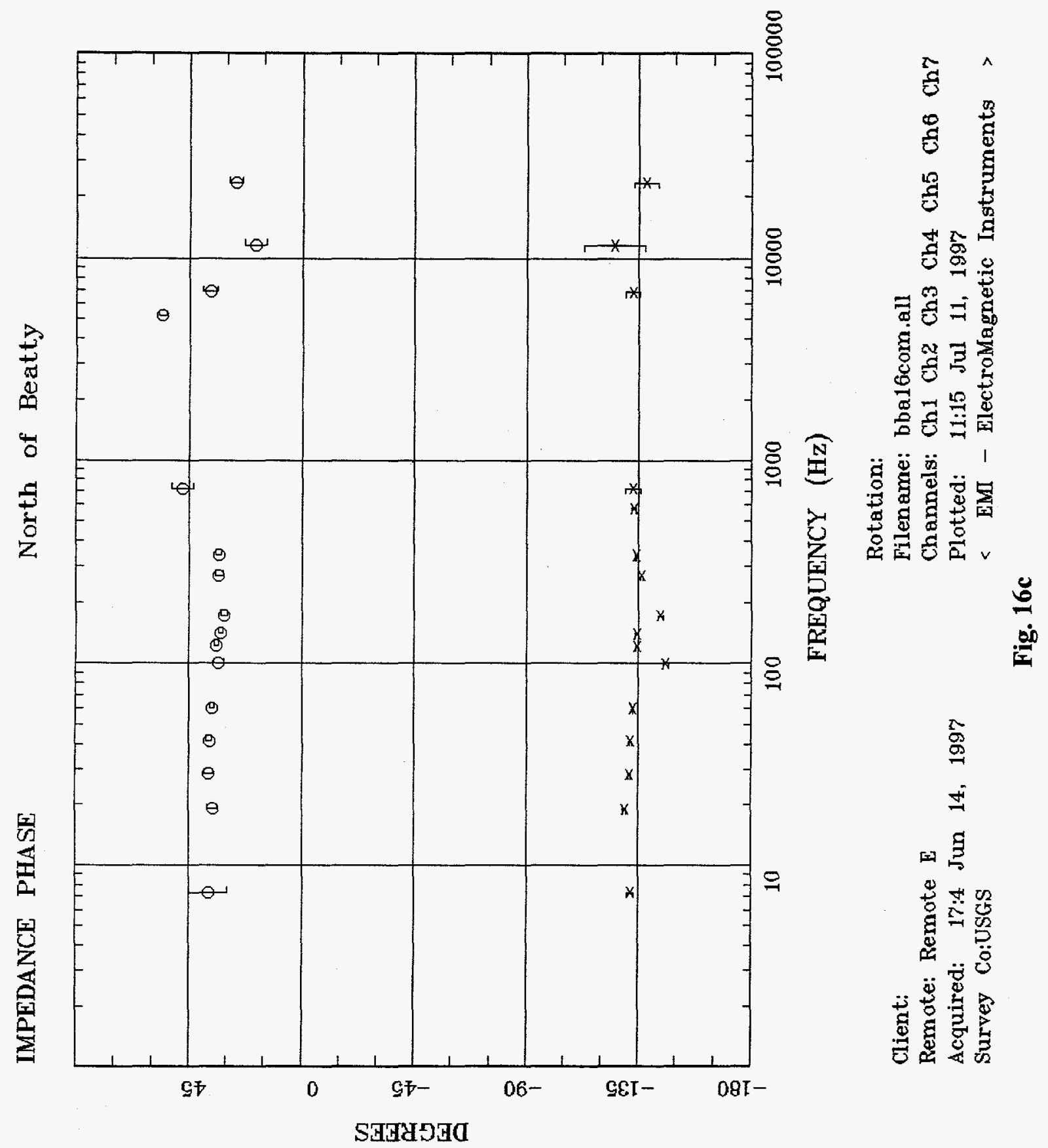




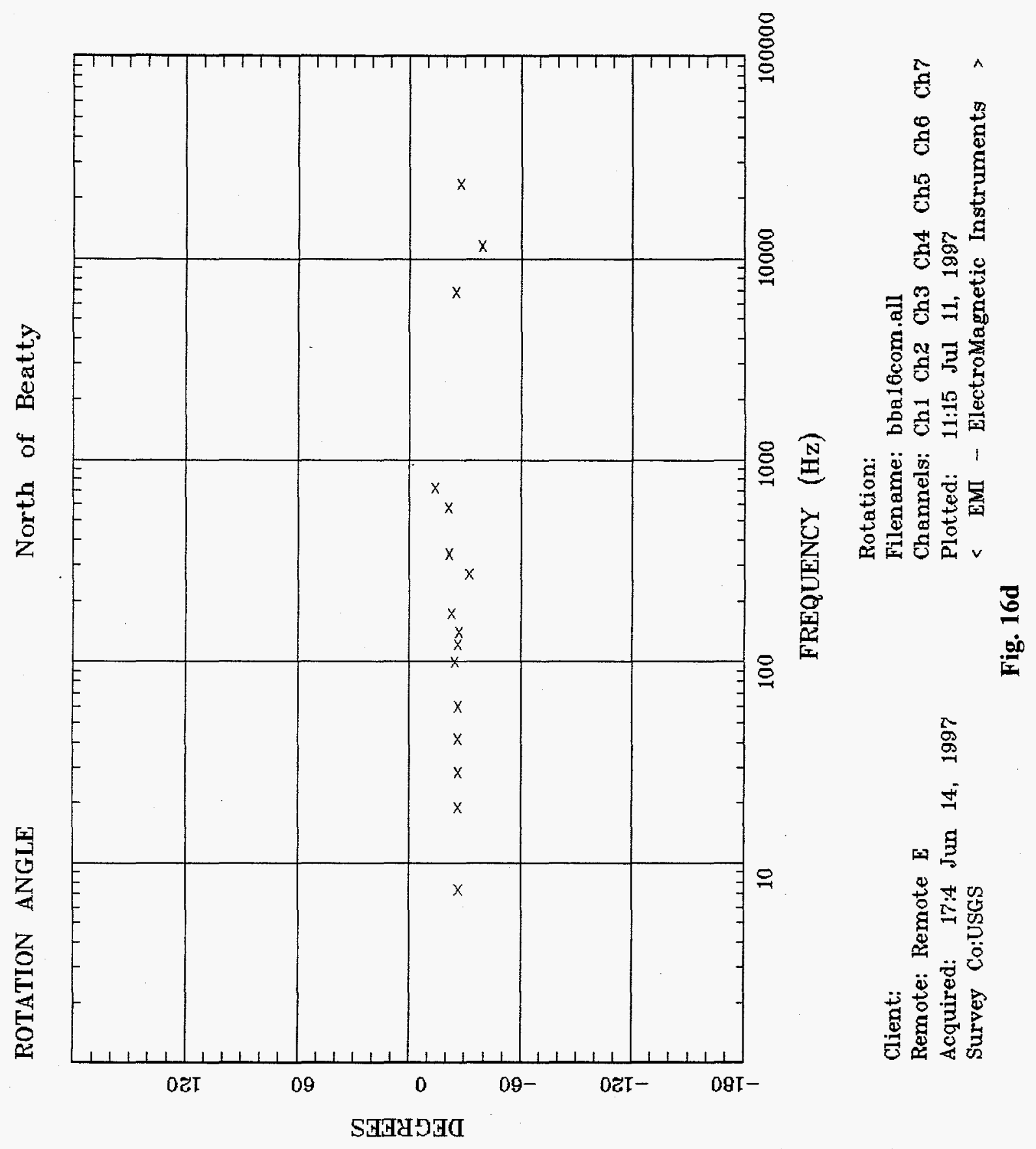


IMPEDANCE SKEW

North of Beatty

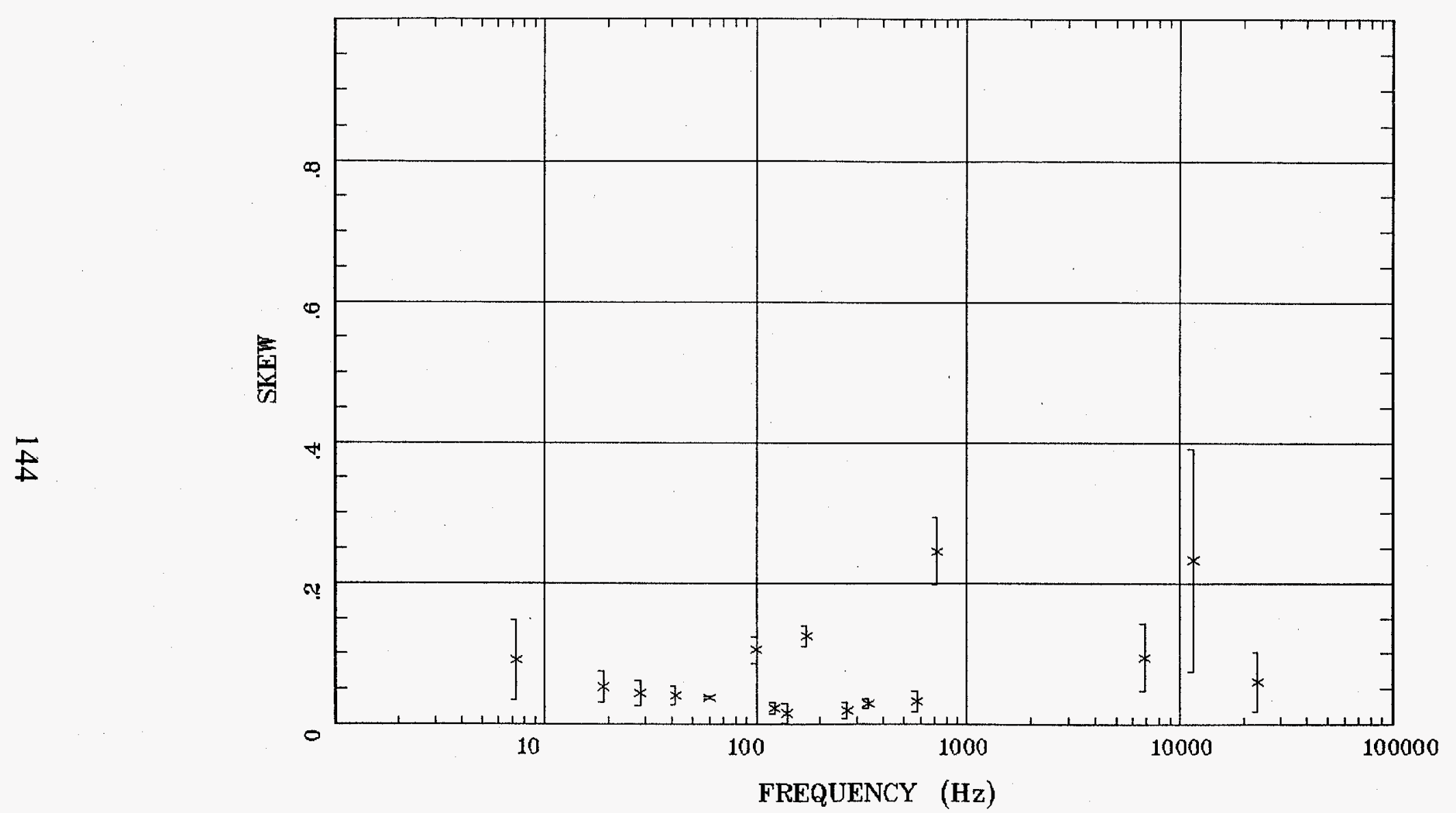

Client:

Remote: Remote E

Acquired: 17:4 Jun 14, 1997 Survey Co:USGS
Rotation:

Filename: bba16com.all

Channels: Ch1 Ch2 Ch3 Ch4 Ch5 Ch6 Ch7

Plotted: 11:15 Jul 11, 1997

< EMI - ElectroMagnetic Instruments >

Fig .16e 


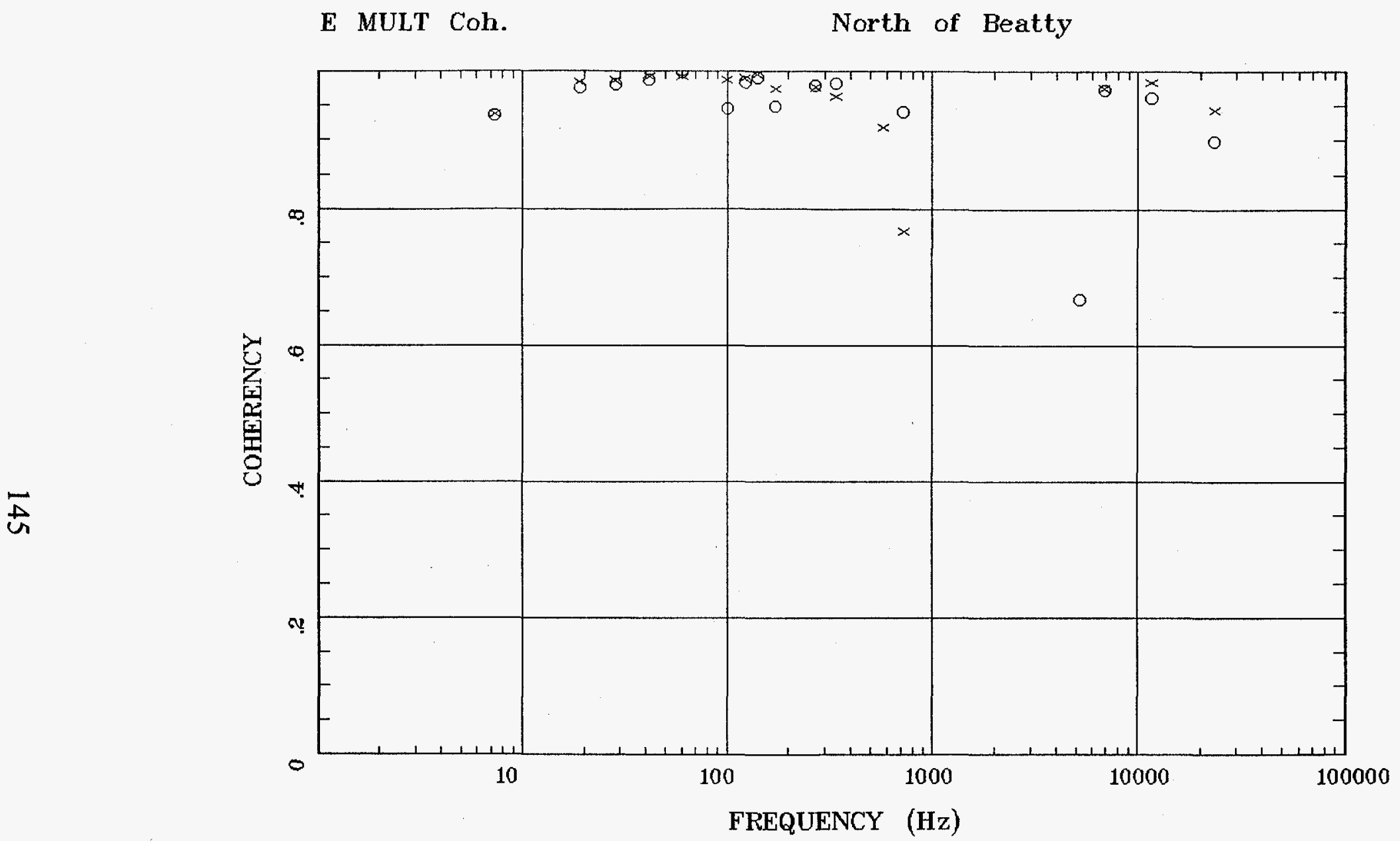

Client:

Remote: Remote E

Acquired: 17:4 Jun 14, 1997

Survey Co:USGS

Rotation:

Filename: bba16com.all

Channels: Ch1 Ch2 Ch3 Ch4 Ch5 Ch6 Ch7

Plotted: 11:15 Jul 11, 1997

< EMI - ElectroMagnetic Instruments >

Fig. 16f 
TIPPER MAGNITUDE

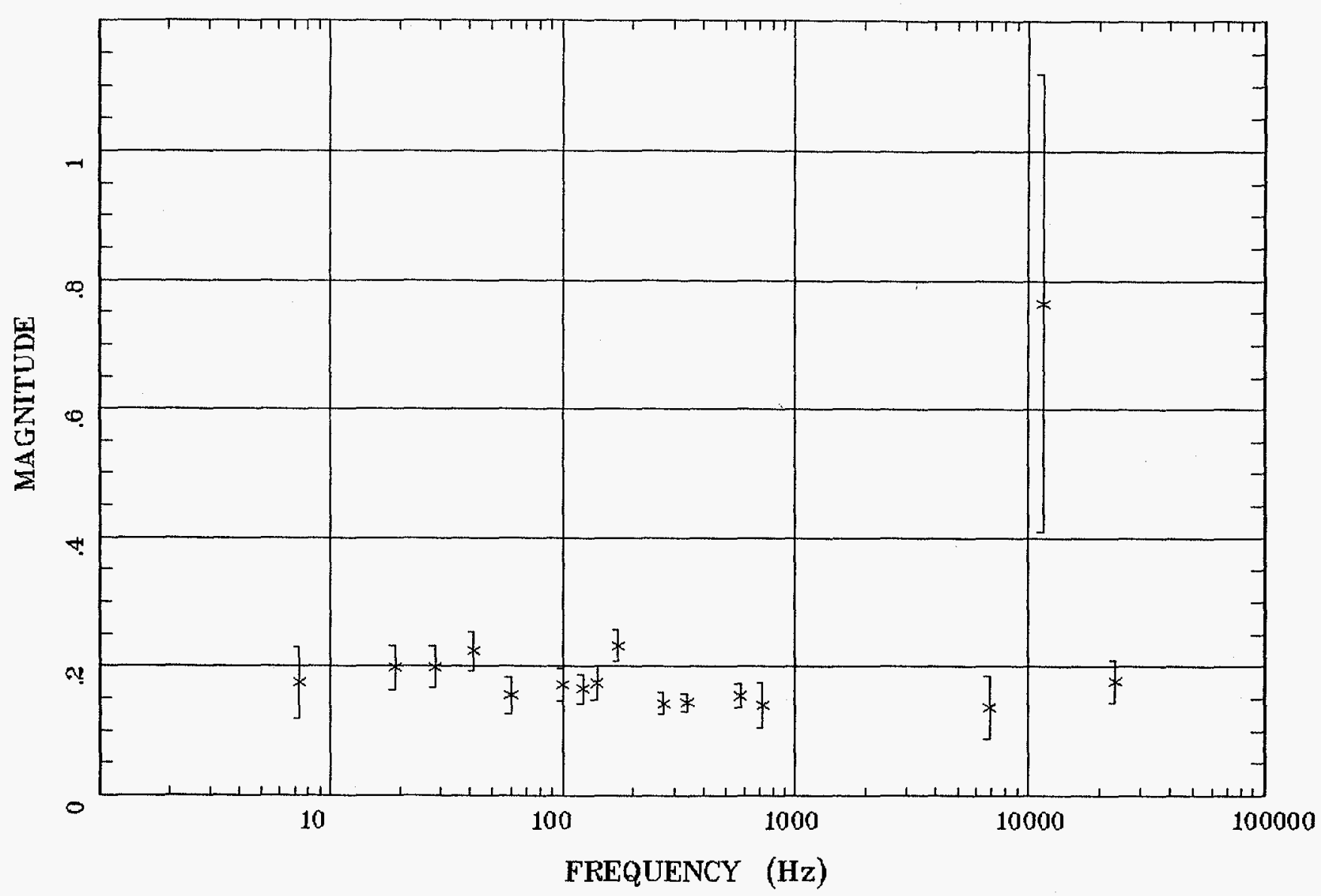

Client:

Remote: Remote $\mathrm{E}$

Acquired: 17:4 Jun 14, 1997 Survey Co:USGS
North of Beatty

\section{Rotation:}

Filename: bba16com.all

Channels: Ch1 Ch2 Ch3 Ch4 Ch5 Ch6 Ch7

Plotted: 11:15 Jul 11, 1997

< EMI - ElectroMagnetic Instruments >

Fig. 16g 


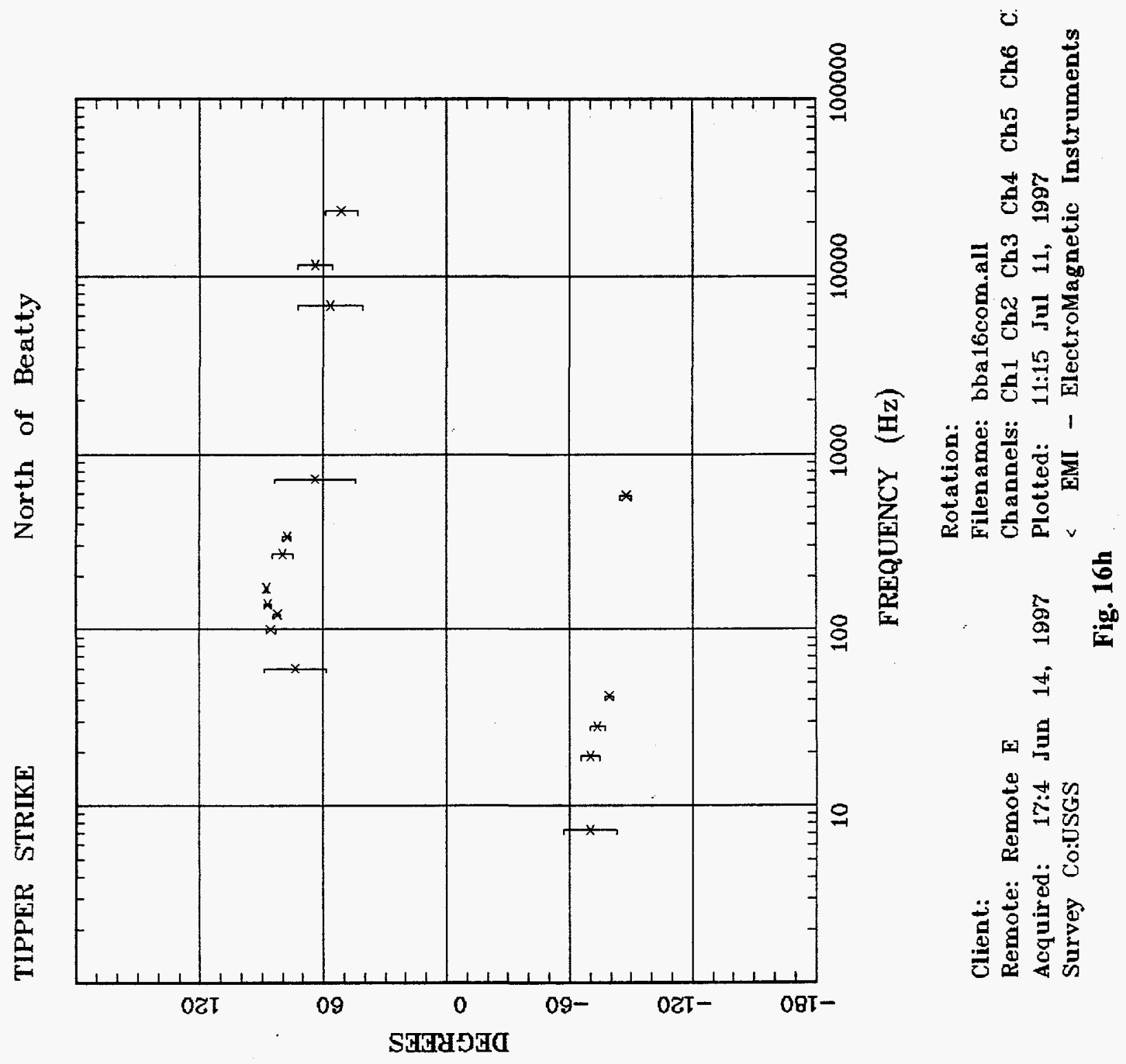




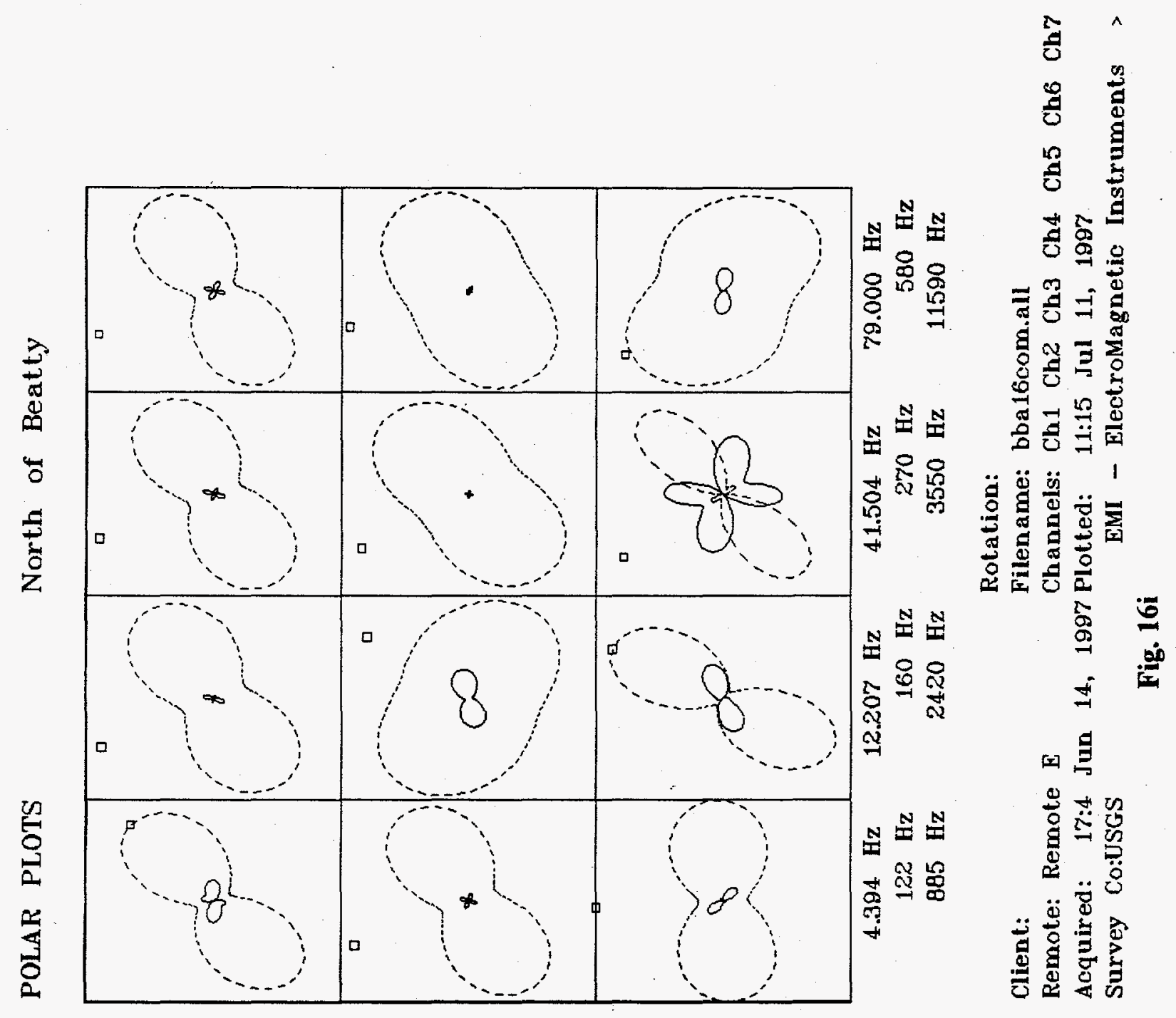

